This document was prepared in conjunction with work accomplished under Contract No. DE-AC09-96SR18500 with the U. S. Department of Energy.

\title{
DISCLAIMER
}

This report was prepared as an account of work sponsored by an agency of the United States Government. Neither the United States Government nor any agency thereof, nor any of their employees, nor any of their contractors, subcontractors or their employees, makes any warranty, express or implied, or assumes any legal liability or responsibility for the accuracy, completeness, or any third party's use or the results of such use of any information, apparatus, product, or process disclosed, or represents that its use would not infringe privately owned rights. Reference herein to any specific commercial product, process, or service by trade name, trademark, manufacturer, or otherwise, does not necessarily constitute or imply its endorsement, recommendation, or favoring by the United States Government or any agency thereof or its contractors or subcontractors. The views and opinions of authors expressed herein do not necessarily state or reflect those of the United States Government or any agency thereof. 
WSRC-TR-2005-00166

SRNL-RPP-2005-00029

Revision 0

\section{CONVENTIONAL WET CHEMISTRY ICP-AES DEVELOPMENT FOR RPP-WTP AY-102/C-106 MELTER FEED SLURRY SIMULANTS - A STATISTICAL REVIEW OF THE RESULTS FROM THE PHASE I STUDY}

Thomas B. Edwards

April 2005

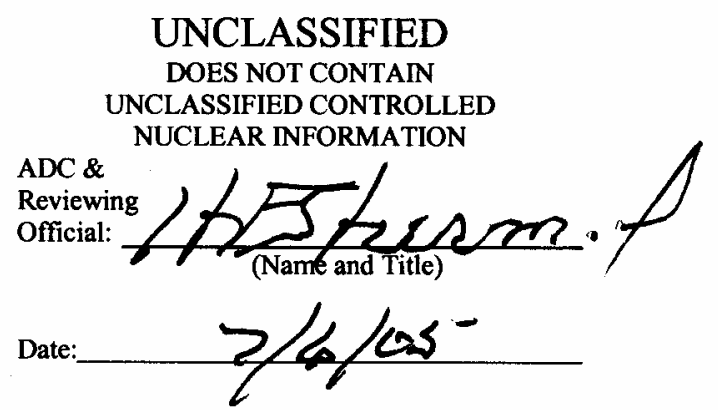

Statistical Consulting Section

Savannah River National Laboratory

Aiken, SC 29808

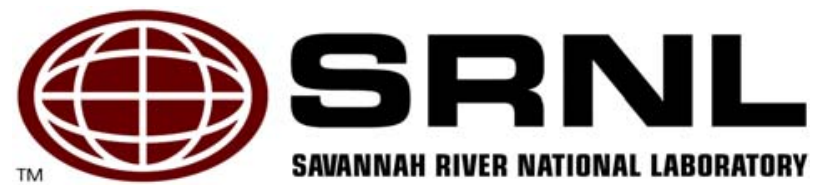


WSRC-TR-2005-00166

SRNL-RPP-2005-00029

Revision 0

\section{DISCLAIMER}

This report was prepared by Westinghouse Savannah River Company (WSRC) for the United States Department of Energy under Contract No. DE-AC09-96SR18500 and is an account of work performed under that contract. Neither the United States Department of Energy, nor WSRC, nor any of their employees makes any warranty, expressed or implied, or assumes any legal liability or responsibility for the accuracy, completeness, or usefulness, of any information, apparatus, or product or process disclosed herein or represents that its use will not infringe privately owned rights. Reference herein to any specific commercial product, process, or service by trademark, name, manufacturer or otherwise does not necessarily constitute or imply endorsement, recommendation, or favoring of same by WSRC or by the United States Government or any agency thereof. The views and opinions of the authors expressed herein do not necessarily state or reflect those of the United States Government or any agency thereof.

\begin{tabular}{|c|}
\hline Printed in the United States of America \\
Prepared For \\
U.S. Department of Energy
\end{tabular}

The Savannah River National Laboratory is operated for the U.S. Department of Energy by Westinghouse Savannah River Company. 
WSRC-TR-2005-00166

SRNL-RPP-2005-00029

Revision 0

Key Words: statistics, dissolution,

ANOVA, mean

Retention: Permanent

\section{CONVENTIONAL WET CHEMISTRY ICP-AES DEVELOPMENT FOR RPP-WTP AY-102/C-106 MELTER FEED SLURRY SIMULANTS - A STATISTICAL REVIEW OF THE RESULTS FROM THE PHASE I STUDY}

Thomas B. Edwards

April 2005

Statistical Consulting Section

Savannah River National Laboratory

Aiken, SC 29808

Prepared for the U.S. Department of Energy Under Contract Number DEAC09-96SR18500
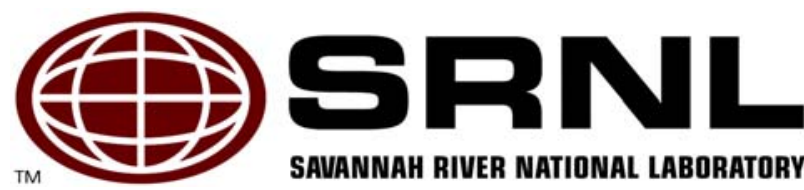

SAUANNAH RIVER NATIONAL LABORATORY 
WSRC-TR-2005-00166

SRNL-RPP-2005-00029

Revision 0

\section{REVIEWS AND APPROVALS}

\section{AUTHOR:}

\section{AUTHOR:}

Ohomeas \& Edwando

T. B. Edwards, Statistical Consulting Section $\frac{5-12-05}{\text { Date }}$

\section{TECHNICAL REVIEWERS:}

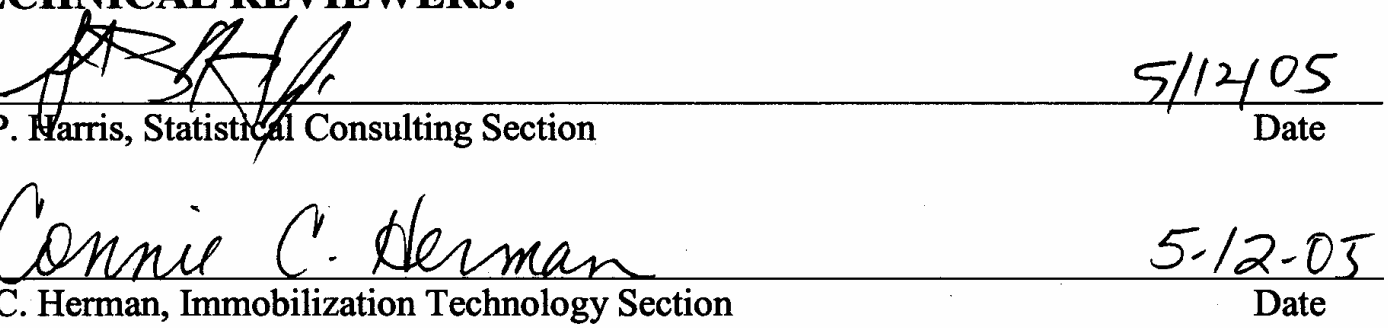

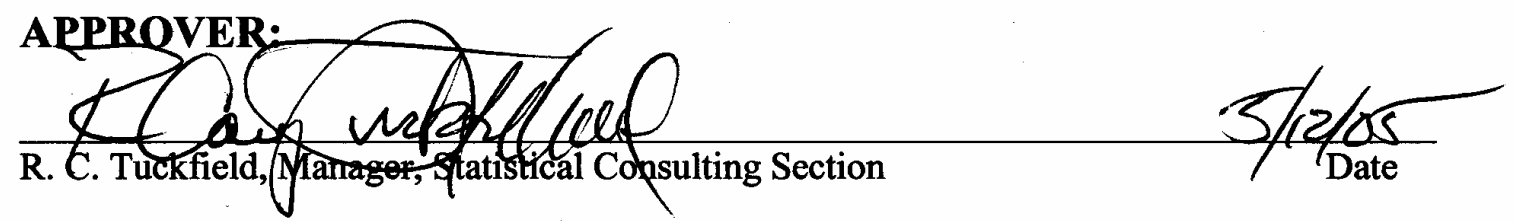




\section{WSRC-TR-2005-00166 \\ SRNL-RPP-2005-00029 \\ Revision 0}

\section{EXECUTIVE SUMMARY}

The River Protection Project (RPP) - Hanford Waste Treatment and Immobilization Plant (WTP) is to prepare and process High Level Waste (HLW) streams into glass waste forms that will meet HLW disposal requirements. Samples of HLW sludge and samples of this sludge mixed with glass-forming chemicals are to be taken and analyzed for process control. Glass characterization from the melter is not included in the scope. The development of viable analytical protocols to provide the required elemental analyses of these samples with rapid turnaround times (before and after addition of the glass-forming chemicals) has been defined as an RPP statement of work for the Analytical Development Section (ADS) of the Savannah River National Laboratory (SRNL). Wet chemistry is serving as the baseline comparison to laser ablation for method development.

One of the simulants used in this study by ADS was AY-102/C-106 melter feed slurry simulant, a simulant used to represent HLW samples after the addition of glass-forming chemicals. Several different dissolution methods were used by ADS in preparing samples of this simulant for elemental analyses by inductively coupled plasma-atomic emission spectroscopy (ICP-AES). The measurements generated by this process were provided to SRNL's Statistical Consulting Section (SCS) for analysis.

The measurement data generated for samples of the RPP-WTP AY-102/C-106 melter feed slurry simulant are presented in this report and the different approaches used to prepare the samples are statistically compared. Comparisons among three of the dissolution methods are highlighted in this analysis. The methods are: sodium peroxide fusion in nickel crucibles, acidification with $\mathrm{HNO}_{3} / \mathrm{HCL}$ at room temperature, and cesium carbonate fusion in zirconium crucibles. A summary table of the measurement averages generated by the three methods is presented. The cesium carbonate fusion method yielded measurements with significantly different mean values from the other two preparation methods for several of the elements.

This report is being provided for informational purposes and supplements the technical report by Coleman and Edwards, "SRNL Phase I Tests of Wet Chemical Non-Dilute/ICP-AES Elemental Analysis Methods for Simulated HLW 2A and HLW 2B Hanford Waste Treatment Plant Samples Interim Report,” WSRC-TR-2005-00169, SRNL-RPP-2005-00023. 
WSRC-TR-2005-00166

SRNL-RPP-2005-00029

Revision 0

This page intentionally left blank. 
WSRC-TR-2005-00166

SRNL-RPP-2005-00029

Revision 0

\section{TABLE OF CONTENTS}

EXECUTIVE SUMMARY iii

LIST OF TABLES vi

LIST OF ACRONYMS

vi

1.0 INTRODUCTION AND BACKGROUND

2.0 RESULTS

2.1 A Description of the Measurement Data

2.2 Preliminary Plots of Measurement Data

2.3 Statistical Comparisons

3.0 CONCLUSIONS

4.0 REFERENCES

APPENDIX Supporting Tables and Exhibits 
WSRC-TR-2005-00166

SRNL-RPP-2005-00029

Revision 0

\section{LIST OF TABLES}

Table 2-1 A Listing of Preparation Methods Utilized with Form of the AY-102/C-106

Melter Feed Slurry Simulant......................................................................................... 2

Table 2-2 Average Recovery ( $\mu \mathrm{g} / \mathrm{g}$ ) by Element for AY-106/C-106 Melter Feed Slurry on a

Dried Solids Basis by Preparation Method ......................................................................... 4

\section{LIST OF ACRONYMS}

$\begin{array}{ll}\text { ADS } & \text { Analytical Development Section } \\ \text { ANOVA } & \text { Analysis of Variance } \\ \text { HLW } & \text { High Level Waste } \\ \text { HSD } & \begin{array}{l}\text { Honestly Significant Difference } \\ \text { JMP }\end{array} \\ & \begin{array}{l}\text { Pronounced “jump.” It’s a statistical software package, a registered trademark } \\ \text { of SAS Institute, Inc. }\end{array} \\ \text { LCS } & \text { Laboratory Control Sample } \\ \text { MFDS } & \text { Melter Feed Dried Solids } \\ \text { MFPV } & \text { Melter Feed Preparation Vessel } \\ \text { MFS } & \text { Melter Feed (Dried) Solids } \\ \text { MFVG } & \text { Melter Feed Vitrified Glass (Vitrified Solids) } \\ \text { RPP } & \text { River Protection Project } \\ \text { SCS } & \text { Statistical Consulting Section } \\ \text { SRNL } & \text { Savannah River National Laboratory } \\ \text { SRS } & \text { Savannah River Site } \\ \text { WTP } & \text { Waste Treatment Plant }\end{array}$




\section{WSRC-TR-2005-00166 \\ SRNL-RPP-2005-00029 \\ Revision 0}

\subsection{INTRODUCTION AND BACKGROUND}

The River Protection Project (RPP) - Hanford Waste Treatment and Immobilization Plant (WTP) is to prepare and process Hanford High Level Waste (HLW) streams into glass waste forms that will meet HLW disposal requirements. The Pretreatment Facility will transfer the HLW to the Melter Feed Preparation Vessel (MFPV) in the vitrification facility as part of this process. In the MFPV, the HLW sludge is to be blended with glass-forming chemicals to produce slurry that is an acceptable melter feed. Variations in the sludge retrieval, pretreatment, and vitrification processes will cause deviations in the nominal melter feed compositions and resulting glass chemistry. Samples of the MFPV are to be taken and analyzed to assess the extent of the deviations in the nominal melter feed. Specifically, MFPV samples are to be taken for each HLW batch transferred to this tank before the addition of the glassforming chemicals; the samples are to be analyzed; the analytical results are to be used to guide the addition of the glass-forming chemicals; and after their addition, another set of MFPV samples is to be taken and analyzed. This final set of analytical measurements is to serve as the basis for the acceptance of the MFPV contents for transfer to the melter. Samples have to be analyzed and the results reported within 9 hours to ensure that feed is available to the melter. The development of viable analytical protocols to provide the required elemental analyses of these MFPV samples (before and after addition of the glassforming chemicals) with rapid turnaround times has been defined as an RPP statement of work [1] for the Savannah River National Laboratory (SRNL). The characterization of the glass waste form produced by the melter is not included in the scope of this work.

In accepting this work, SRNL's Analytical Development Section (ADS) issued a Task Technical and Quality Assurance Plan [2] that covered a set of activities that were to be conducted by ADS using simulants previously prepared for the RPP-WTP program as the first phase (Phase I) of study. One of the simulants was melter feed slurry containing AY-102/C-106 simulant with glass-forming chemicals. Different dissolution methods were used by ADS in preparing samples of this simulant (on a dried solids and a vitrified solids basis) for elemental analyses by inductively coupled plasma-atomic emission spectroscopy (ICP-AES). Wet chemistry is serving as the baseline comparison to laser ablation for method development. The measurements generated by this process were provided to SRNL's Statistical Consulting Section (SCS) for analysis.

The purpose of this technical report is to present a statistical interpretation of the analytical data generated by ADS and provide a comparison of the results. This report is being provided for informational purposes and supplements the technical report by Coleman and Edwards, "SRNL Phase I Tests of Wet Chemical Non-Dilute/ICP-AES Elemental Analysis Methods for Simulated HLW 2A and HLW 2B Hanford Waste Treatment Plant Samples - Interim Report,” WSRC-TR-2005-00169, SRNL-RPP-200500023. The statistical analyses were carried out using JMP ${ }^{\circledR}$ Version 5.1.2 [3] from SAS Institute, Inc. The validation and verification for this statistical software is provided in [4].

\subsection{RESULTS}

In this section the measurement data generated for samples of the RPP-WTP AY-102/C-106 melter feed slurry simulant are presented and the different approaches used to prepare the samples are statistically compared. A summary table of three of the dissolution methods used to analyze the samples of this material on a dried-solids basis is highlighted in the discussion that follows. 


\section{WSRC-TR-2005-00166 \\ SRNL-RPP-2005-00029 \\ Revision 0}

\subsection{A Description of the Measurement Data}

The measurement data from this ADS study are provided in Table A1 and Table A2 in the Appendix. Two tables are used to cover all of the elemental analyses that were recorded during this investigation. Table A1 provides elemental concentrations in parts per million ( $\mathrm{ppm}$ ) for the following elements: silver $(\mathrm{Ag})$, aluminum (Al), boron (B), barium (Ba), calcium (Ca), cadmium (Cd), cerium (Ce), copper (Cu), iron $(\mathrm{Fe})$, potassium $(\mathrm{K})$, lanthanum $(\mathrm{La})$, lithium $(\mathrm{Li})$, magnesium $(\mathrm{Mg})$, manganese $(\mathrm{Mn})$, and molybdenum (Mo). Table A2 provides elemental concentrations in ppm for the following elements: sodium (Na), nickel (Ni), phosphorous (P), lead (Pb), sulfur (S), antimony (Sb), silicon (Si), tin (Sn), strontium (Sr), thorium (Th), titanium (Ti), tellurium (Tl), uranium (U), yttrium (Y), zinc (Zn), and zirconium ( $\mathrm{Zr})$. In addition to the elements, there are columns identifying the form of the simulant used for the analysis, the preparation method and type of crucible (if a crucible was used) employed to dissolve the sample and the type of sample involved. There were three different preparation/crucible combinations investigated for the simulant on a dried-solids basis and on a vitrified-solids basis. Table 2-1 provides a listing of these combinations.

Table 2-1 A Listing of Preparation Methods Utilized with Form of the AY-102/C-106 Melter Feed Slurry Simulant

\begin{tabular}{|c|c|}
\hline Form of Simulant & Preparation Method/Type of Crucible \\
\hline AY-102/C-106 MFDS (melter feed dried-solids) & Cesium Carbonate Fusion/Pt \\
\hline AY-102/C-106 MFDS (melter feed dried-solids) & Sodium Peroxide Fusion/Ni \\
\hline AY-102/C-106 MFS (melter feed dried-solids) & Acidification $\mathrm{wNO}_{3} / \mathrm{HCl}$ \\
\hline AY-102/C-106 MFVG (melter feed vitrified-solids) & Cesium Carbonate Fusion/Pt \\
\hline AY-102/C-106 MFVG (melter feed vitrified-solids) & Potassium Carbonate/Pt \\
\hline AY-102/C-106 MFVG (melter feed vitrified-solids) & Sodium Peroxide Fusion/Ni \\
\hline
\end{tabular}

The types of samples for this study included: blanks, trial samples (samples of the simulant), laboratory control samples (LCS), and spiked samples (samples of the simulant spiked with known amounts of one or more elements). The first three types of samples are the focus in the analyses that follow and these are used to provide insight into the relative performances of the dissolution methods investigated as part of this Phase I effort. However, note that the trial samples are the primary focus of the statistical comparisons. Measurements that were below the detection limit of the analytical process (indicated by a " $<$ ” in Tables A1 and A2) were replaced by half of the detection limit in the analyses that follow. Also, note that some values were not reported for some elements as indicated by a missing value (a blank) or the letters "NA" (for Not Available) in the tables. An example of this situation is provided by the lack of Ni measurements for samples dissolved in a nickel crucible.

\subsection{Preliminary Plots of Measurement Data}

Exhibit A1 in the Appendix provides a plot of the measurements by type of sample across all of the forms of the simulant and preparation methods by element in the sequence that the data were reported by ADS.

\subsection{Statistical Comparisons}

Exhibit A2 in the Appendix provides statistical comparisons among the preparation methods and forms of the simulant for each element for each type of sample. The statistical comparisons include a one-way analysis of variance (ANOVA) for these data (when enough data are available) investigating for a difference in the mean recoveries over these sets of measurements for the specified element. A statistically significant difference in the mean recoveries (at the $5 \%$ significance level) is indicated by a 
WSRC-TR-2005-00166

SRNL-RPP-2005-00029

Revision 0

value of 0.05 or less for "Prob $>F$ " in these exhibits. If no value is provided for "Prob $>F$ " in the exhibit, there was not enough data to conduct the statistical test. The means and standard deviations of the sets of measurements for each form of the simulant for each preparation method are also given in this exhibit.

Table 2-2 provides summary information for the measurements generated using the three preparation methods for the dried-solids form of this melter feed slurry simulant along with the LCS and blank samples. Exhibit A3 in the Appendix provides a more specific set of comparisons among the samples of the dried solids form of this melter feed slurry prepared by these three methods. In this exhibit, JMP's Tukey-Kramer HSD (honestly significant difference) test is used to investigate for all differences among the mean measurements over the three preparation methods for each element. The results of this test for each element is a symmetric matrix covering the preparation methods used with positive entries in the matrix indicating a pair of preparation methods that yielded mean measurements that are significantly different.

In Table 2-2, if the cesium carbonate fusion preparation yielded measurements for the trial samples in Exhibit A3 in the Appendix that indicated a statistically significant different mean from the means of both of the other preparation methods, that entry is shaded. 
WSRC-TR-2005-00166

SRNL-RPP-2005-00029

Revision 0

Table 2-2 Average Recovery $(\mu \mathrm{g} / \mathrm{g})$ by Element for AY-102/C-106 Melter Feed Slurry on a Dried Solids Basis by Preparation Method

\begin{tabular}{|c|c|c|c|c|c|c|c|c|c|}
\hline \multirow[b]{2}{*}{ Element } & \multicolumn{3}{|c|}{ Trial (Simulant) Samples } & \multicolumn{3}{|c|}{ LCSs (Laboratory Control Samples) } & \multicolumn{3}{|c|}{ Blanks } \\
\hline & $\begin{array}{c}\text { Sodium } \\
\text { Peroxide } \\
\text { Fusion/Ni } \\
\text { Crucible } \\
\end{array}$ & $\begin{array}{c}\text { Acidification } \\
\text { with } \\
\mathrm{HNO}_{3} / \mathrm{HCL} \\
\end{array}$ & $\begin{array}{c}\text { Cesium } \\
\text { Carbonate } \\
\text { Fusion/Pt } \\
\text { Crucible }\end{array}$ & $\begin{array}{c}\text { Sodium } \\
\text { Peroxide } \\
\text { Fusion/Ni } \\
\text { Crucible }\end{array}$ & $\begin{array}{c}\text { Acidification } \\
\text { with } \\
\mathrm{HNO}_{3} / \mathrm{HCL} \\
\end{array}$ & $\begin{array}{c}\text { Cesium } \\
\text { Carbonate } \\
\text { Fusion/Pt } \\
\text { Crucible } \\
\end{array}$ & $\begin{array}{l}\text { Sodium } \\
\text { Peroxide } \\
\text { Fusion/Ni } \\
\text { Crucible }\end{array}$ & $\begin{array}{c}\text { Acidification } \\
\text { with } \\
\mathrm{HNO}_{3} / \mathrm{HCL}\end{array}$ & $\begin{array}{c}\text { Cesium } \\
\text { Carbonate } \\
\text { Fusion/Pt } \\
\text { Crucible }\end{array}$ \\
\hline $\mathrm{Ag}$ & 407.8 & 187.0 & 642.8 & 33.0 & 33.0 & 83.2 & 17.2 & \begin{tabular}{|l|}
17.2 \\
\end{tabular} & 17.2 \\
\hline $\mathrm{Al}$ & 10450.0 & 7380.0 & 10425.0 & 22450.0 & 24700.0 & 23600.0 & 156.5 & 156.5 & 156.5 \\
\hline $\mathrm{B}$ & 10200.0 & 11700.0 & 10850.0 & 23300.0 & 24900.0 & 23700.0 & 62.0 & 62.0 & 62.0 \\
\hline $\mathrm{Ba}$ & 442.0 & 323.3 & 352.0 & 1055.0 & 987.5 & 933.0 & 95.8 & 13.8 & 43.6 \\
\hline $\mathrm{Ca}$ & 1867.5 & 1426.7 & 1387.5 & 12300.0 & 12750.0 & 10150.0 & 349.0 & 55.0 & 55.0 \\
\hline $\mathrm{Cd}$ & 26.1 & 25.0 & 30.7 & 69.3 & 69.3 & 90.5 & 36.1 & 36.1 & 36.1 \\
\hline $\mathrm{Ce}$ & 797.8 & 767.0 & 667.0 & 953.0 & 1245.0 & 525.0 & 209.5 & 209.5 & 209.5 \\
\hline $\mathrm{Cr}$ & 916.5 & 370.0 & 405.8 & 1550.0 & 1200.0 & 632.5 & 420.0 & 33.1 & 33.1 \\
\hline $\mathrm{Cu}$ & 57.7 & 76.6 & 105.0 & 39.7 & 39.7 & 51.8 & 20.7 & 20.7 & 20.7 \\
\hline $\mathrm{Fe}$ & 37575.0 & 38100.0 & 36725.0 & 101500.0 & 10300.0 & 99100.0 & 87.5 & 7.1 & 7.1 \\
\hline $\mathrm{K}$ & 3377.5 & 2306.7 & 2697.5 & 28900.0 & 29900.0 & 24050.0 & 1960.0 & 835.0 & 835.0 \\
\hline $\mathrm{La}$ & 400.8 & 419.7 & 376.3 & 210.0 & 424.0 & 177.0 & 70.5 & 70.5 & 70.5 \\
\hline $\mathrm{Li}$ & 4800.0 & 4843.3 & 4420.0 & 16550.0 & 17400.0 & 14700.0 & 36.1 & 36.1 & 36.1 \\
\hline $\mathrm{Mg}$ & 403.3 & 380.3 & 397.3 & 5200.0 & 5295.0 & 5135.0 & 12.1 & 12.1 & 12.1 \\
\hline $\mathrm{Mn}$ & 8235.0 & 8506.7 & 8002.5 & 14850.0 & 15050.0 & 14450.0 & 5.2 & 5.2 & 5.2 \\
\hline Mo & 910.8 & 150.0 & 130.4 & 1650.0 & 1785.0 & 384.8 & 806.0 & 153.0 & 153.0 \\
\hline $\mathrm{Na}$ &. & 32266.7 & 30525.0 &. &. & 80200.0 &. & 373.0 & 373.0 \\
\hline $\mathrm{Ni}$ & . & 1246.7 & 1212.0 &. &. & 8350.0 &. & 49.9 & 49.9 \\
\hline $\mathrm{P}$ & 932.5 & 891.7 & 1098.8 & 2480.0 & 2480.0 & 3240.0 & 1290.0 & 1290.0 & 1290.0 \\
\hline $\mathrm{Pb}$ & 2265.0 & 1806.7 & 1026.3 & 1747.5 & 1747.5 & 2282.5 & 910.0 & 910.0 & 910.0 \\
\hline $\mathrm{S}$ & 275.6 & & & 735.0 & & & 381.5 & & \\
\hline $\mathrm{Sb}$ & 1905.0 & 212.3 & 229.5 & 3630.0 & 3870.0 & 674.0 & 1670.0 & 98.0 & 98.0 \\
\hline $\mathrm{Si}$ & 86025.0 & 5206.7 & 82725.0 & 232000.0 & 238000.0 & 223500.0 & 89.5 & 89.5 & 89.5 \\
\hline Sn & 574.4 & 550.2 & 676.3 & 1530.0 & 1530.0 & 2000.0 & 795.0 & 795.0 & 795.0 \\
\hline $\mathrm{Sr}$ & 673.0 & 548.3 & 535.8 & 2675.0 & 2625.0 & 2120.0 & 80.9 & 27.5 & 27.5 \\
\hline Th & 66.8 &. &. & 159.0 &. & & 67.9 &. &. \\
\hline $\mathrm{Ti}$ & 8.7 & 2.2 & 17.5 & 7060.0 & 7135.0 & 6990.0 & 12.1 & 3.2 & 3.2 \\
\hline $\mathrm{Tl}$ & 343.3 &. &. & 944.0 &. &. & 375.0 &. &. \\
\hline $\mathrm{U}$ & 141.6 &. &. & 578.5 & . &. & 259.0 &. &. \\
\hline $\mathrm{Y}$ & 67.5 & 1460.0 & 74.0 & 330.3 & 330.3 & 431.5 & 172.0 & 172.0 & 172.0 \\
\hline $\mathrm{Zn}$ & 4270.0 & 3310.0 & 2988.3 & 2315.0 & 2670.0 & 204.5 & 1130.0 & 17.2 & 17.2 \\
\hline $\mathrm{Zr}$ & 654.0 & 279.7 & 513.0 & 1145.0 & 1105.0 & 876.5 & 97.2 & 9.2 & 9.2 \\
\hline
\end{tabular}

Multiple digestion methods were tested because no single digestion method is suitable for all of the elemental analyses needed for WTP process control. Although several combinations of two digestion methods would supply the needed elemental analyses, a tandem dissolution approach of a sodium peroxide fusion in a nickel crucible and a warm-acid method has certain advantages in terms of combining a very aggressive digestion (the sodium peroxide fusion) with a very rapid method (the warmacid method). The former method provides analyses for all elements of interest except $\mathrm{Na}$ and $\mathrm{Ni}$ while the later method, which may not completely dissolve the sample, does dissolve the $\mathrm{Na}$ and $\mathrm{Ni}$ elements to provide analyses for this pair of elements that cannot be obtained from the sodium peroxide fusion. This tandem approach was then compared with a cesium carbonate fusion in platinum crucibles. This later approach was investigated because of its potential as a single-digestion method for the elemental analysis of the WTP process samples. 
WSRC-TR-2005-00166

SRNL-RPP-2005-00029

Revision 0

The results from Table 2-2 indicate that the cesium carbonate fusion method yielded measurements with mean values that were statistically smaller at the $5 \%$ significance level than the mean values for the measurements from the other two preparation methods for: $\mathrm{Fe}, \mathrm{Li}, \mathrm{Mn}, \mathrm{Na}$, and $\mathrm{Pb}$, while the mean values for $\mathrm{Ag}, \mathrm{Cu}$, and $\mathrm{Ti}$ for the cesium carbonate fusion method were statistically larger at the 5\% significance level than the mean values for the other two preparation methods. The cesium carbonate fusion measurement averages for $\mathrm{B}, \mathrm{Ba}, \mathrm{K}$, and $\mathrm{Zr}$ were statistically different from the means of both of the other preparation methods, even though the cesium carbonate fusion measurement averages fell in between the averages of the measurements of the other two preparation methods. For example, consider Fe, the analyte with the highest measured concentrations for the simulant samples that showed a statistically significant difference among the mean values. The average cesium carbonate fusion value (36,725 $\mu \mathrm{g} / \mathrm{g}$ ) for Fe is $2.3 \%$ smaller than the average sodium peroxide fusion value $(37,575 \mu \mathrm{g} / \mathrm{g})$ and $3.6 \%$ smaller than the average warm acid value (38,100 $\mu \mathrm{g} / \mathrm{g})$. However, accuracy and precision requirements for the WTP elemental analyses have not yet been established. Thus, even though there are statistically significant differences, it is not clear that these point to analytical issues at this stage of the study. As seen in the plots of Exhibit A2, the precision was quite good for many of the elements on many of the sets of analyses, which leads to comparisons among the methods that are sensitive to small differences for those elements.

\subsection{CONCLUSIONS}

The measurement data generated for samples of the RPP-WTP AY-102/C-106 simulant after the addition of glass forming chemicals are presented in this report and the different approaches used to prepare the samples are statistically compared. Comparisons among three of the dissolution methods are highlighted in this analysis. The methods are: sodium peroxide fusion in nickel crucibles, acidification with $\mathrm{HNO}_{3} / \mathrm{HCL}$ at room temperature, and cesium carbonate fusion in zirconium crucibles. A summary table of the measurement averages generated by the three methods is presented. The cesium carbonate fusion method yielded measurements with mean values that were significantly different from the other two preparation methods for several of the elements. 
WSRC-TR-2005-00166

SRNL-RPP-2005-00029

Revision 0

\subsection{REFERENCES}

[1] “WSRC-SRNL NTP Wet Chemistry \& Laser Ablation Testing,” CCN 0918450, August 2004.

[2] Herman, C. C., C. J. Coleman, and K. E. Zeigler, “Task Technical and Quality Assurance Plan for Conventional Wet Chemistry and Laser Ablation ICP-AES Development,” WSRC-TR-2004-00447, SRNL-RPP-2004-00073, Revision 0, October 2004.

[3] SAS Institute, Inc., JMP ${ }^{\mathrm{TM}}$ Version 5 Statistics and Graphics Guide, SAS Institute, Inc., Cary NC, 2002.

[4] Baker, R. A., T. B. Edwards, S. P. Harris, C. D. Harvel, and E. P. Shine, "Software QA Plan and Verification \& Validation for Commercial Statistical Packages Utilized by the Statistical Consulting Section of SRNL (U),” WSRC-RP-99-00422, Revision 4, May 2005. 
WSRC-TR-2005-00166

SRNL-RPP-2005-00029

Revision 0

\section{APPENDIX \\ Supporting Tables and Exhibits}


WSRC-TR-2005-00166

SRNL-RPP-2005-00029

Revision 0

This page intentionally left blank. 
WSRC-TR-2005-00166

SRNL-RPP-2005-00029

Revision 0

Table A1. First Set of Elemental Analyses of Samples of WTP-RPP AY-102/C-106 Melter Feed Slurry

\begin{tabular}{|c|c|c|c|c|c|c|c|c|c|c|c|c|c|c|c|c|c|c|}
\hline Simulant & Prep/Crucible & $\begin{array}{l}\text { Type of } \\
\text { Sample } \\
\end{array}$ & \begin{tabular}{|c|}
$\mathrm{Ag}$ \\
$(\mathrm{ppm})$
\end{tabular} & $\begin{array}{c}\mathrm{Al} \\
(\mathrm{ppm})\end{array}$ & \begin{tabular}{|c|}
$\mathrm{B}$ \\
$(\mathrm{ppm})$
\end{tabular} & $\begin{array}{c}\mathrm{Ba} \\
(\mathrm{ppm}) \\
\end{array}$ & \begin{tabular}{|c|}
$\mathrm{Ca}$ \\
$(\mathrm{ppm})$
\end{tabular} & $\begin{array}{c}\mathrm{Cd} \\
(\mathrm{ppm}) \\
\end{array}$ & $\begin{array}{c}\mathrm{Ce} \\
(\mathrm{ppm}) \\
\end{array}$ & $\begin{array}{c}\mathrm{Cr} \\
(\mathrm{ppm})\end{array}$ & \begin{tabular}{|c|}
$\mathrm{Cu}$ \\
$(\mathrm{ppm})$
\end{tabular} & $\begin{array}{c}\begin{array}{c}\mathrm{Fe} \\
(\mathrm{ppm})\end{array} \\
\end{array}$ & $\begin{array}{c}\mathrm{K} \\
(\mathrm{ppm})\end{array}$ & \begin{tabular}{|c|}
$\mathrm{La}$ \\
$(\mathrm{ppm})$
\end{tabular} & $\begin{array}{c}\mathrm{Li} \\
(\mathrm{ppm})\end{array}$ & $\begin{array}{c}\mathrm{Mg} \\
(\mathrm{ppm}) \\
\end{array}$ & $\begin{array}{c}\mathrm{Mn} \\
(\mathrm{ppm})\end{array}$ & $\begin{array}{c}\begin{array}{c}\text { Mo } \\
\text { (ppm) }\end{array} \\
\end{array}$ \\
\hline AY-102/C-106 MFS & Acidification w $\mathrm{HNO} / \mathrm{HCl} /$ & Blank & $<34.4$ & $<<313$ & $<124$ & $<27.5$ & $<110$ & $<72.2$ & $<419$ & $<66.2$ & $<41.3$ & $<14.2$ & $<1670$ & $<141$ & $<72.2$ & $<24.1$ & $<10.3$ & $<306$ \\
\hline AY-102/C-106 MFS & Acidification w $\mathrm{HNO} / \mathrm{HCl} /$ & Trial & 256 & 7130 & 11600 & 316 & 1420 & $<62.1$ & 879 & 351 & 77 & 38200 & 2490 & 448 & 4860 & 365 & 8540 & $<264$ \\
\hline AY-102/C-106 MFS & Acidification w $\mathrm{HNO} / \mathrm{HCl} /$ & Trial & 125 & 7140 & 11800 & 328 & 1430 & $<42.9$ & 706 & 377 & 76 & 38000 & 2150 & 404 & 4790 & 388 & 8490 & 223 \\
\hline AY-102/C-106 MFS & Acidification w HNO3/HCl/ & Trial & 180 & 7870 & 11700 & 326 & 1430 & $<44.7$ & 716 & 382 & 76.8 & 38100 & 2280 & 407 & 4880 & 388 & 8490 & $<190$ \\
\hline AY-102/C-106 MFS & Acidification w $\mathrm{HNO} / \mathrm{HCl} /$ & LCS & $<66.9$ & 24600 & 24800 & 1020 & 12800 & $<140$ & 1310 & 1400 & $<80.3$ & 10300 & 30500 & 430 & 17300 & 5280 & 15000 & 2080 \\
\hline AY-102/C-106 MFS & Acidification w HNO3/HCl/ & LCS & $<65.2$ & 24800 & 25000 & 955 & 12700 & $<137$ & 1180 & 1000 & $<78.3$ & 10300 & 29300 & 418 & 17500 & 5310 & 15100 & 1490 \\
\hline AY-102/C-106 MFDS & Cesium Carbonate Fusion/Pt & Blank & $<34.4$ & $<313$ & $<124$ & 43.6 & $<110$ & $<72.2$ & $<419$ & $<66.2$ & $<41.3$ & $<14.2$ & $<1670$ & $<141$ & $<72.2$ & $<24.1$ & $<10.3$ & $<306$ \\
\hline AY-102/C-106 MFDS & Cesium Carbonate Fusion/Pt & Trial & 636 & 10200 & 10900 & 343 & 1450 & $<53.1$ & 624 & 416 & 105 & 36400 & 2600 & 358 & 4330 & 400 & 7920 & $<293$ \\
\hline AY-102/C-106 MFDS & Cesium Carbonate Fusion/Pt & Trial & 526 & 10500 & 10900 & 361 & 1340 & $<69.7$ & 736 & 382 & 111 & 37200 & 2800 & 397 & 4450 & 404 & 8110 & $<228$ \\
\hline AY-102/C-106 MFDS & Cesium Carbonate Fusion/Pt & Trial & 732 & 10500 & 10700 & 346 & 1340 & $<61.9$ & 646 & 410 & 101 & 36500 & 2710 & 369 & 4440 & 389 & 7950 & $<263$ \\
\hline AY-102/C-106 MFDS & Cesium Carbonate Fusion/Pt & Trial & 677 & 10500 & 10900 & 358 & 1420 & $<61.0$ & 662 & 415 & 103 & 36800 & 2680 & 381 & 4460 & 396 & 8030 & $<259$ \\
\hline AY-102/C-106 MFDS & Cesium Carbonate Fusion/Pt & LCS & 122 & 23600 & 23600 & 921 & 10200 & $<176$ & $<1020$ & 592 & $<101$ & 99000 & 23300 & $<344$ & 14700 & 5100 & 14400 & $<748$ \\
\hline AY-102/C-106 MFDS & Cesium Carbonate Fusion/Pt & LCS & $<88.7$ & 23600 & 23800 & 945 & 10100 & $<186$ & $<1080$ & 673 & $<106$ & 99200 & 24800 & $<364$ & 14700 & 5170 & 14500 & $<791$ \\
\hline AY-102/C-106 MFDS & Sodium Peroxide Fusion/Ni & Blank & $<34.4$ & $<313$ & $<124$ & 95.8 & 349 & $<72.2$ & $<419$ & 420 & $<41.3$ & $<175$ & 1960 & $<141$ & $<72.2$ & $<24.1$ & $<10.3$ & 806 \\
\hline AY-102/C-106 MFDS & Sodium Peroxide Fusion/Ni & Trial & 288 & 10200 & 10100 & 431 & 1690 & $<56.4$ & 772 & 798 & 49.8 & 37300 & 3480 & 397 & 4720 & 392 & 8180 & 764 \\
\hline AY-102/C-106 MFDS & Sodium Peroxide Fusion/Ni & Trial & 425 & 10600 & 10100 & 425 & 1830 & $<55.5$ & 818 & 834 & 55.7 & 37900 & 3170 & 408 & 4840 & 406 & 8280 & 811 \\
\hline AY-102/C-106 MFDS & Sodium Peroxide Fusion/Ni & Trial & 452 & 10500 & 10300 & 445 & 2030 & $<52.6$ & 874 & 944 & 62.4 & 37700 & 3450 & 404 & 4790 & 408 & 8280 & 998 \\
\hline AY-102/C-106 MFDS & Sodium Peroxide Fusion/Ni & Trial & 466 & 10500 & 10300 & 467 & 1920 & $<44.2$ & 727 & 1090 & 62.9 & 37400 & 3410 & 394 & 4850 & 407 & 8200 & 1070 \\
\hline AY-102/C-106 MFDS & Sodium Peroxide Fusion/Ni & LCS & $<66.9$ & 22400 & 23700 & 1090 & 12400 & $<140$ & 932 & 1790 & $<80.3$ & 102000 & 26900 & $<274$ & 16500 & 5190 & 14800 & 1670 \\
\hline AY-102/C-106 MFDS & Sodium Peroxide Fusion/Ni & LCS & $<65.2$ & 22500 & 22900 & 1020 & 12200 & $<137$ & 974 & 1310 & $<78.3$ & 101000 & 30900 & 283 & 16600 & 5210 & 14900 & 1630 \\
\hline AY-102/C-106 MFVG & Sodium Peroxide Fusion/Ni & Blank & $<34.4$ & $<313$ & $<124$ & 95.8 & 349 & $<72.2$ & $<419$ & 420 & $<41.3$ & $<175$ & 1960 & $<141$ & $<72.2$ & $<24.1$ & $<10.3$ & 806 \\
\hline AY-102/C-106 MFVG & Sodium Peroxide Fusion/Ni & Trial & 502 & 10600 & 10100 & 407 & 1750 & $<54.0$ & 813 & 750 & 60.8 & 37900 & 3540 & 401 & 4720 & 413 & 8310 & 579 \\
\hline AY-102/C-106 MFVG & Sodium Peroxide Fusion/Ni & Trial & 477 & 10600 & 9980 & 645 & 1700 & $<54.7$ & 892 & 1950 & 89.7 & 37600 & 3500 & 412 & 4690 & 304 & 8270 & 2270 \\
\hline AY-102/C-106 MFVG & Sodium Peroxide Fusion/Ni & Trial & 405 & 10600 & 9930 & 498 & 1930 & $<49.8$ & 785 & 1170 & 62.6 & 37900 & 3690 & 412 & 4870 & 406 & 8310 & 1210 \\
\hline AY-102/C-106 MFVG & Sodium Peroxide Fusion/Ni & Trial & 494 & 11000 & \begin{tabular}{|c|}
9760 \\
\end{tabular} & 525 & 2200 & $<63.1$ & 851 & 1320 & 63.2 & 39000 & 3890 & 434 & 4910 & 410 & 8520 & 1490 \\
\hline AY-102/C-106 MFVG & Sodium Peroxide Fusion/Ni & LCS & $<66.9$ & 22400 & 23700 & 1090 & 12400 & $<140$ & 932 & 1790 & $<80.3$ & 102000 & 26900 & $<274$ & 16500 & 5190 & 14800 & 1670 \\
\hline AY-102/C-106 MFVG & Sodium Peroxide Fusion/Ni & LCS & $<65.2$ & 22500 & 22900 & 1020 & 12200 & $<137$ & 974 & 1310 & $<78.3$ & 101000 & 30900 & 283 & 16600 & 5210 & 14900 & 1630 \\
\hline AY-102/C-106 MFVG & Cesium Carbonate Fusion/Pt & Blank & $<19.8$ & $<180$ & $<71.3$ & 35.2 & $<63.4$ & $<41.6$ & $<242$ & $<38.1$ & $<23.8$ & $<8.20$ & 1020 & $<81.2$ & $<41.6$ & $<13.9$ & $<5.94$ & $<177$ \\
\hline AY-102/C-106 MFVG & Cesium Carbonate Fusion/Pt & Trial & 528 & 11200 & 10700 & 369 & 1380 & 46.8 & 614 & 429 & 91.1 & 37100 & 3250 & 359 & 4520 & 418 & 8120 & $<161$ \\
\hline AY-102/C-106 MFVG & Cesium Carbonate Fusion/Pt & Trial & 499 & 11300 & 11100 & 381 & 1390 & 49.8 & 720 & 430 & 94.3 & 37400 & 3580 & 390 & 4550 & 416 & 8190 & $<202$ \\
\hline AY-102/C-106 MFVG & Cesium Carbonate Fusion/Pt & Trial & 543 & 11300 & 10600 & 371 & 1400 & 46.1 & 648 & 423 & 92.8 & 37400 & 3010 & 372 & 4580 & 429 & 8200 & $<170$ \\
\hline AY-102/C-106 MFVG & Cesium Carbonate Fusion/Pt & Trial & 600 & 11100 & 10800 & 364 & 1360 & 45 & 589 & 451 & 90.6 & 36900 & 3270 & 357 & 4450 & 408 & 8040 & $<177$ \\
\hline AY-102/C-106 MFVG & Cesium Carbonate Fusion/Pt & Trial & 596 & 11100 & 11100 & 358 & 1370 & 44.6 & 578 & 433 & 87.8 & 36700 & 2660 & 354 & 4480 & 412 & 8040 & $<141$ \\
\hline AY-102/C-106 MFVG & Cesium Carbonate Fusion/Pt & LCS & 125 & 23700 & 24700 & 928 & 10500 & $<115$ & $<666$ & 679 & 67.1 & 99900 & 25500 & $<224$ & 15100 & 5200 & 14600 & $<486$ \\
\hline AY-102/C-106 MFVG & Cesium Carbonate Fusion/Pt & LCS & $<57.7$ & 23900 & 24600 & 946 & 10500 & $<121$ & $<703$ & 578 & $<69.2$ & 100000 & 25400 & $<236$ & 15200 & 5260 & 14700 & $<514$ \\
\hline AY-102/C-106 MFVG & Potassium Carbonate/Pt & Blank & $<26.8$ & $<244$ & $<96.5$ & $<21.5$ & $<85.8$ & $<56.3$ & $<327$ & $<51.6$ & $<32.2$ & $<11.1$ & & $<110$ & 78 & $<18.8$ & $<8.04$ & $<239$ \\
\hline AY-102/C-106 MFVG & Potassium Carbonate/Pt & Trial & 443 & 11100 & 10800 & 332 & 1320 & $<42.3$ & 666 & 411 & 86.4 & 36300 & & 382 & 4560 & 402 & 7900 & $<180$ \\
\hline AY-102/C-106 MFVG & Potassium Carbonate/Pt & Trial & 397 & 11000 & 10800 & 332 & 1280 & $<63.0$ & 783 & 406 & 91 & 35300 & & 395 & 4400 & 382 & 7830 & $<267$ \\
\hline AY-102/C-106 MFVG & Potassium Carbonate/Pt & Trial & 472 & 11000 & 10800 & 337 & 1310 & $<48.2$ & 755 & 380 & 93.4 & 36000 & & 399 & 4550 & 396 & 7860 & $<205$ \\
\hline
\end{tabular}


WSRC-TR-2005-00166

SRNL-RPP-2005-00029

Revision 0

Table A2. Second Set of Elemental Analyses of Samples of WTP-RPP AY-102/C-106 Melter Feed Slurry

\begin{tabular}{|c|c|c|c|c|c|c|c|c|c|c|c|c|c|c|c|c|c|c|}
\hline Simulant & Prep/Crucible & Type of Sample & $\begin{array}{c}\mathrm{Na} \\
(\mathrm{ppm})\end{array}$ & $\begin{array}{c}\mathrm{Ni} \\
(\mathrm{ppm})\end{array}$ & $\begin{array}{c}\mathrm{P} \\
(\mathrm{ppm})\end{array}$ & $\begin{array}{c}\mathrm{Pb} \\
(\mathrm{ppm})\end{array}$ & $\begin{array}{c}\mathrm{S} \\
(\mathrm{ppm})\end{array}$ & $\begin{array}{c}\begin{array}{c}\mathrm{Sb} \\
(\mathrm{ppm})\end{array}\end{array}$ & $\begin{array}{c}\mathrm{Si} \\
(\mathrm{ppm})\end{array}$ & $\begin{array}{c}\text { Sn } \\
(\mathrm{ppm})\end{array}$ & $\begin{array}{c}\mathrm{Sr} \\
(\mathrm{ppm})\end{array}$ & $\begin{array}{c}\text { Th } \\
(\mathrm{ppm})\end{array}$ & $\begin{array}{c}\mathrm{Ti} \\
(\mathrm{ppm})\end{array}$ & $\begin{array}{c}\mathrm{Tl} \\
(\mathrm{ppm})\end{array}$ & $\underset{(\mathrm{ppm})}{\mathrm{U}}$ & $\begin{array}{c}\mathrm{Y} \\
(\mathrm{ppm})\end{array}$ & $\begin{array}{c}\mathrm{Zn} \\
(\mathrm{ppm})\end{array}$ & $\begin{array}{c}\mathrm{Zr} \\
(\mathrm{ppm})\end{array}$ \\
\hline AY-102/C-106 MFS & Acidification w HNO3/HCl/ & Blank & $<746$ & $<99.7$ & $<2580$ & $<1820$ & & $<196$ & $<<179$ & $<1590$ & $<55.0$ & & $<6.32$ & & & $<<344$ & $<34.4$ & $<18.3$ \\
\hline AY-102/C-106 MFS & Acidification w HNO3/HCl/ & Trial & 32300 & 1260 & $<2220$ & 1740 & & 232 & 4590 & $<1370$ & 545 & & $<5.43$ & & & $<296$ & 3330 & 277 \\
\hline AY-102/C-106 MFS & Acidification w $\mathrm{HNO} / \mathrm{HCl} /$ & Trial & 32300 & 1220 & $<1530$ & 1760 & & 193 & 4780 & $<945$ & 547 & & $<3.75$ & & & $<204$ & 3300 & 304 \\
\hline AY-102/C-106 MFS & Acidification w HNO3/HCl/ & Trial & 32200 & 1260 & $<1600$ & 1920 & & 212 & 6250 & $<986$ & 553 & & $<3.91$ & & & 4130 & 3300 & 258 \\
\hline AY-102/C-106 MFS & Acidification w HNO3/HCl/ & LCS & NA & $\mathrm{Ni}$ & $<5020$ & $<3540$ & & 4530 & 237000 & $<3100$ & 2640 & & 7100 & & & $<669$ & 3220 & 1020 \\
\hline AY-102/C-106 MFS & Acidification w HNO3/HCl/ & LCS & NA & NA & $<4900$ & $<3450$ & & 3210 & 239000 & $<3020$ & 2610 & & 7170 & & & $<652$ & 2120 & 1190 \\
\hline AY-102/C-106 MFDS & Cesium Carbonate Fusion/Pt & Blank & $<746$ & $<99.7$ & $<2580$ & $<1820$ & & $<196$ & $<179$ & $<1590$ & $<55.0$ & & $<6.32$ & & & $<344$ & $<34.4$ & $<18.3$ \\
\hline AY-102/C-106 MFDS & Cesium Carbonate Fusion/Pt & Trial & 30300 & 1190 & $<2470$ & $<1700$ & & 191 & 78500 & $<1170$ & 545 & & 18.8 & & & $<253$ & 2961 & 492 \\
\hline AY-102/C-106 MFDS & Cesium Carbonate Fusion/Pt & Trial & 30800 & 1258 & $<1920$ & $<1350$ & & 262 & 84800 & $<1530$ & 537 & & 15.7 & & & $<331$ & 3042 & 531 \\
\hline AY-102/C-106 MFDS & Cesium Carbonate Fusion/Pt & Trial & 30400 & 1210 & $<2220$ & 1730 & & 215 & 83200 & $<1370$ & 519 & & 17.5 & & & 1.94 & 2960 & 515 \\
\hline AY-102/C-106 MFDS & Cesium Carbonate Fusion/Pt & Trial & 30600 & 1190 & $<2180$ & $<1700$ & & 250 & 84400 & $<1340$ & 542 & & 18 & & & 1.94 & 2990 & 514 \\
\hline AY-102/C-106 MFDS & Cesium Carbonate Fusion/Pt & LCS & 80300 & 8190 & $<6300$ & $<4440$ & & 534 & 222000 & $<3890$ & 2100 & & 6980 & & & $<839$ & 199 & 864 \\
\hline AY-102/C-106 MFDS & Cesium Carbonate Fusion/Pt & LCS & 80100 & 8510 & $<6660$ & $<4690$ & & 814 & 225000 & $<4110$ & 2140 & & 7000 & & & $<887$ & 210 & 889 \\
\hline AY-102/C-106 MFDS & Sodium Peroxide Fusion/Ni & Blank & & & $<2580$ & $<1820$ & $<763$ & 1670 & $<179$ & $<1590$ & 80.9 & 67.9 & $<24.1$ & 375 & 259 & $<344$ & 1130 & 97.2 \\
\hline AY-102/C-106 MFDS & Sodium Peroxide Fusion/Ni & Trial & & & $<2020$ & 2070 & $<596$ & 1660 & 85200 & $<1240$ & 630 & 63.9 & $<18.8$ & 311 & $<134$ & $<268$ & 4040 & 634 \\
\hline AY-102/C-106 MFDS & Sodium Peroxide Fusion/Ni & Trial & & & $<1980$ & 2340 & $<586$ & 1540 & 87100 & $<1220$ & 656 & 55.5 & $<18.5$ & 382 & 284 & $<264$ & 4030 & 642 \\
\hline AY-102/C-106 MFDS & Sodium Peroxide Fusion/Ni & Trial & & & $<1880$ & 2230 & $<556$ & 1950 & 86300 & $<1160$ & 713 & 72.2 & $<17.5$ & 366 & 163 & 1.93 & 4350 & 664 \\
\hline AY-102/C-106 MFDS & Sodium Peroxide Fusion/Ni & Trial & & & $<1580$ & 2420 & $<467$ & 2470 & 85500 & $<975$ & 693 & 75.5 & $<14.7$ & 314 & $<105$ & 1.96 & 4660 & 676 \\
\hline AY-102/C-106 MFDS & Sodium Peroxide Fusion/Ni & LCS & & & $<5020$ & $<3540$ & $<1490$ & 4260 & 231000 & $<3100$ & 2710 & 175 & 7050 & 1100 & 679 & $<669$ & 2790 & 1110 \\
\hline AY-102/C-106 MFDS & Sodium Peroxide Fusion/Ni & LCS & & & $<4900$ & $<3450$ & $<1450$ & 3000 & 233000 & $<3020$ & 2640 & 143 & 7070 & 788 & 478 & $<652$ & 1840 & 1180 \\
\hline AY-102/C-106 MFVG & Sodium Peroxide Fusion/Ni & Blank & & & $<2580$ & $<1820$ & $<763$ & 1670 & $<179$ & $<1590$ & 80.9 & 67.9 & $<24.1$ & 375 & 259 & $<344$ & 1130 & 97.2 \\
\hline AY-102/C-106 MFVG & Sodium Peroxide Fusion/Ni & Trial & & & $<1930$ & 2100 & $<570$ & 1170 & 86400 & $<1190$ & 652 & 49.7 & $<18.0$ & 318 & 199 & $<257$ & 3750 & 639 \\
\hline AY-102/C-106 MFVG & Sodium Peroxide Fusion/Ni & Trial & & & $<1960$ & 3030 & $<578$ & 5450 & 85200 & 1370 & 682 & 186 & $<18.2$ & 647 & 207 & $<260$ & 6880 & 782 \\
\hline AY-102/C-106 MFVG & Sodium Peroxide Fusion/Ni & Trial & & & $<1780$ & 2540 & $<526$ & 2840 & 86000 & $<1100$ & 706 & 106 & $<16.6$ & 439 & 189 & 1.93 & 4980 & 695 \\
\hline AY-102/C-106 MFVG & Sodium Peroxide Fusion/Ni & Trial & & & $<2260$ & 2590 & $<667$ & 3330 & 88300 & $<1390$ & 775 & 103 & $<21.0$ & 494 & 200 & 1.93 & 5400 & 742 \\
\hline AY-102/C-106 MFVG & Sodium Peroxide Fusion/Ni & LCS & & & $<5020$ & $<3540$ & $<1490$ & 4260 & 231000 & $<3100$ & 2710 & 175 & 7050 & 1100 & 679 & $<669$ & 2790 & 1110 \\
\hline AY-102/C-106 MFVG & Sodium Peroxide Fusion/Ni & LCS & & & $<4900$ & $<3450$ & $<1450$ & 3000 & 233000 & $<3020$ & 2640 & 143 & 7070 & 788 & 478 & $<652$ & 1840 & 1180 \\
\hline AY-102/C-106 MFVG & Cesium Carbonate Fusion/Pt & Blank & 524 & $<57.4$ & $<1490$ & $<1050$ & & $<113$ & $<103$ & $<917$ & $<31.7$ & & $<3.64$ & & & $<198$ & $<19.8$ & $<10.5$ \\
\hline AY-102/C-106 MFVG & Cesium Carbonate Fusion/Pt & Trial & 32400 & 1160 & $<1360$ & $<958$ & & 226 & 85400 & $<839$ & 532 & & 19.1 & & & $<181$ & 3090 & 530 \\
\hline AY-102/C-106 MFVG & Cesium Carbonate Fusion/Pt & Trial & 33100 & 1190 & $<1700$ & $<1200$ & & 287 & 85900 & $<1050$ & 531 & & 20 & & & $<227$ & 3130 & 530 \\
\hline AY-102/C-106 MFVG & Cesium Carbonate Fusion/Pt & Trial & 32400 & 1210 & $<1430$ & $<1010$ & & 249 & 85900 & $<881$ & 541 & & 18.4 & & & $<190$ & 3090 & 539 \\
\hline AY-102/C-106 MFVG & Cesium Carbonate Fusion/Pt & Trial & 31900 & 1210 & $<1490$ & $<1050$ & & 224 & 83900 & $<918$ & 531 & & 18.3 & & & 2.5 & 3030 & 494 \\
\hline AY-102/C-106 MFVG & Cesium Carbonate Fusion/Pt & Trial & 31700 & 1180 & $<1190$ & $<839$ & & 182 & 83900 & $<734$ & 535 & & 18.9 & & & 2.52 & 3030 & 501 \\
\hline AY-102/C-106 MFVG & Cesium Carbonate Fusion/Pt & LCS & 85500 & 8330 & $<4100$ & $<2890$ & & 581 & 230000 & $<2530$ & 2130 & & 7020 & & & $<546$ & 220 & 904 \\
\hline AY-102/C-106 MFVG & Cesium Carbonate Fusion/Pt & LCS & 85600 & 8400 & $<4330$ & $<3050$ & & 709 & 232000 & $<2670$ & 2150 & & 7070 & & & $<577$ & 211 & 928 \\
\hline AY-102/C-106 MFVG & Potassium Carbonate/Pt & Blank & $<582$ & $<77.8$ & $<2010$ & $<1420$ & & $<153$ & $<139$ & $<1240$ & $<42.9$ & & $<4.93$ & & & $<268$ & $<26.8$ & $<14.2$ \\
\hline AY-102/C-106 MFVG & Potassium Carbonate/Pt & Trial & 29000 & 1090 & $<1510$ & 1520 & & 242 & 82000 & $<933$ & 543 & & 18.9 & & & 1.93 & 2960 & 494 \\
\hline AY-102/C-106 MFVG & Potassium Carbonate/Pt & Trial & 29100 & 565 & $<2250$ & $<1590$ & & 260 & 81300 & $<1390$ & 514 & & 20.1 & & & 1.92 & 2930 & 483 \\
\hline AY-102/C-106 MFVG & Potassium Carbonate/Pt & Trial & 29000 & 929 & $<1720$ & 1610 & & 297 & 82400 & $<1060$ & 533 & & 18.8 & & & 1.93 & 2970 & 504 \\
\hline
\end{tabular}


WSRC-TR-2005-00166

SRNL-RPP-2005-00029

Revision 0

\section{Exhibit A1. Elemental Measurements Plotted by Sample Type in Reported Sequence}

Type of Sample=Blank

Individual Measurement of Ag

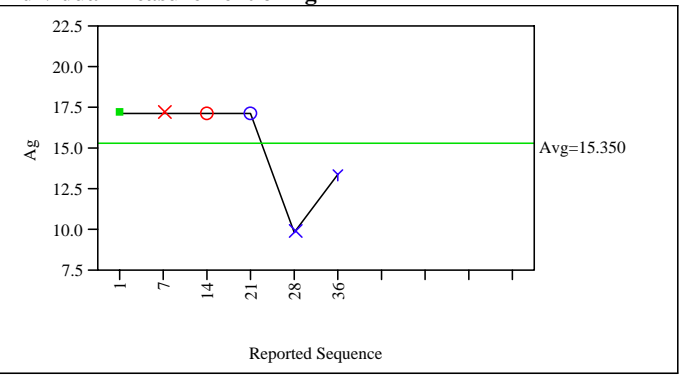

Individual Measurement of A

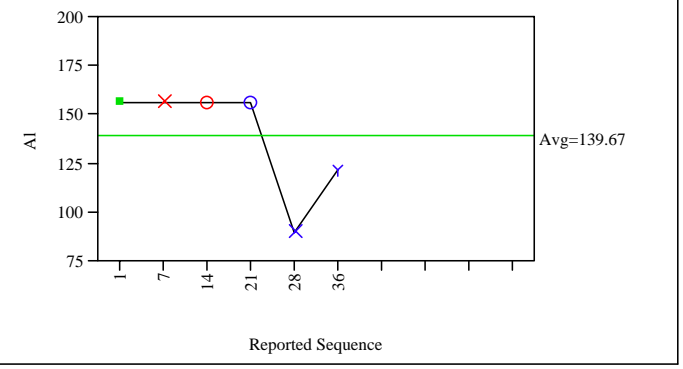

Individual Measurement of B

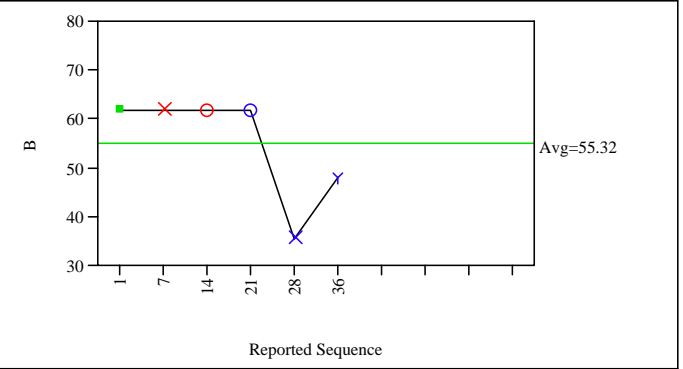

Individual Measurement of Ba

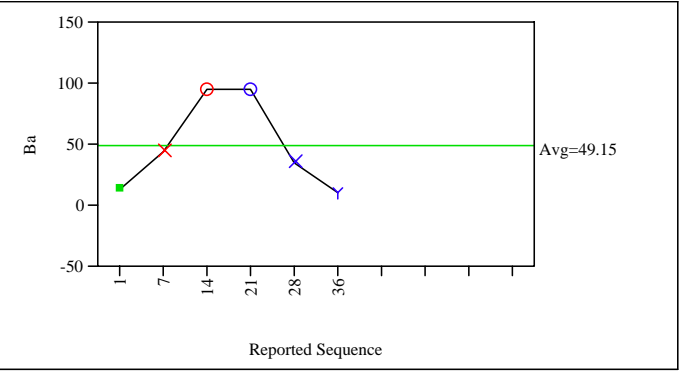

Individual Measurement of $\mathrm{Ca}$

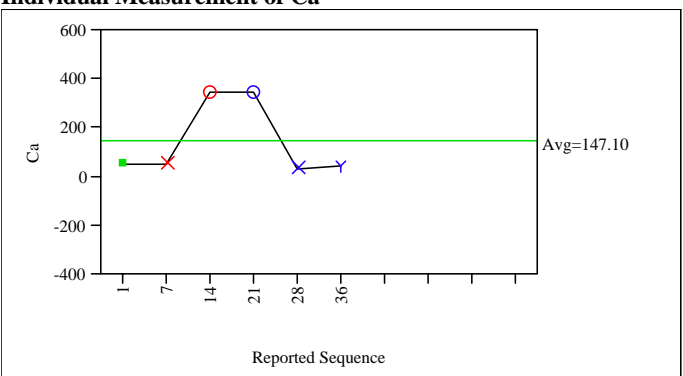

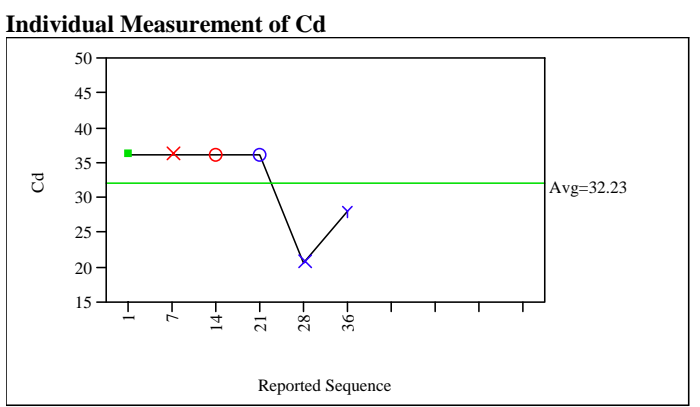
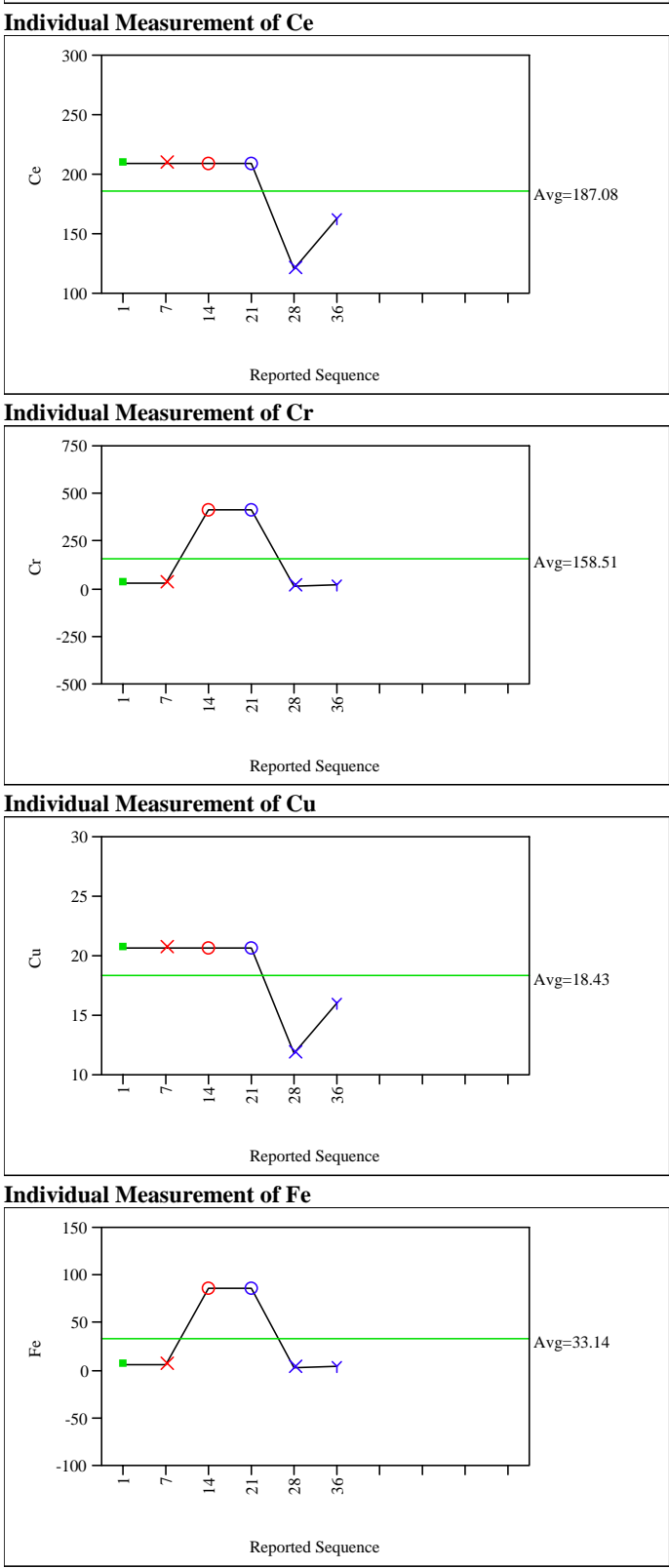
WSRC-TR-2005-00166

SRNL-RPP-2005-00029

Revision 0

\section{Exhibit A1. Elemental Measurements Plotted by Sample Type in Reported Sequence}

Type of Sample=Blank

Individual Measurement of $\mathrm{K}$

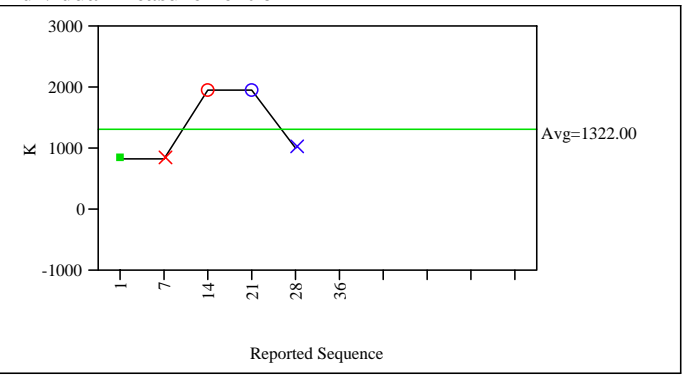

Individual Measurement of La

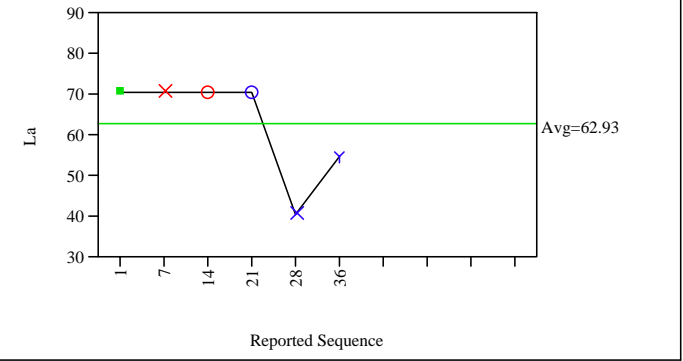

Individual Measurement of $\mathrm{Li}$

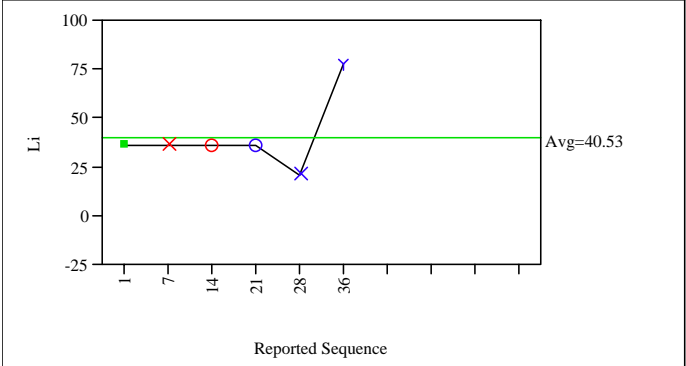

Individual Measurement of $\mathbf{M g}$

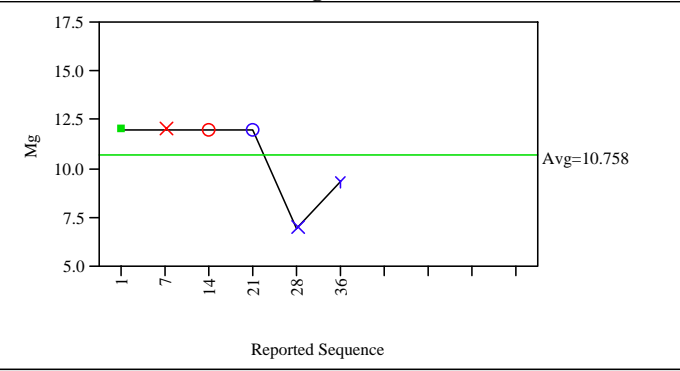

Individual Measurement of Mn

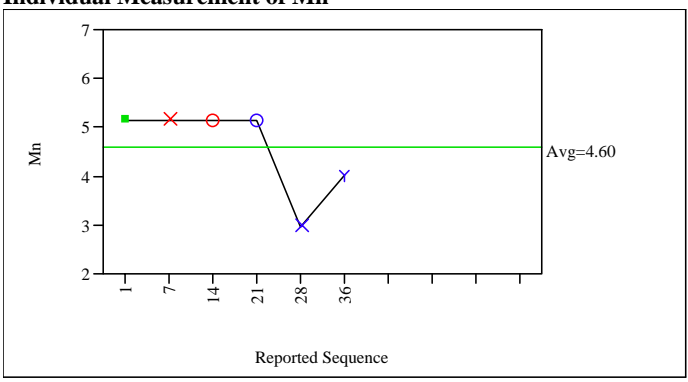

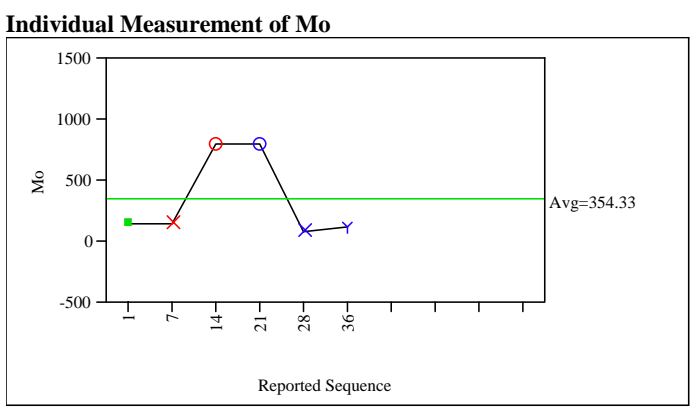
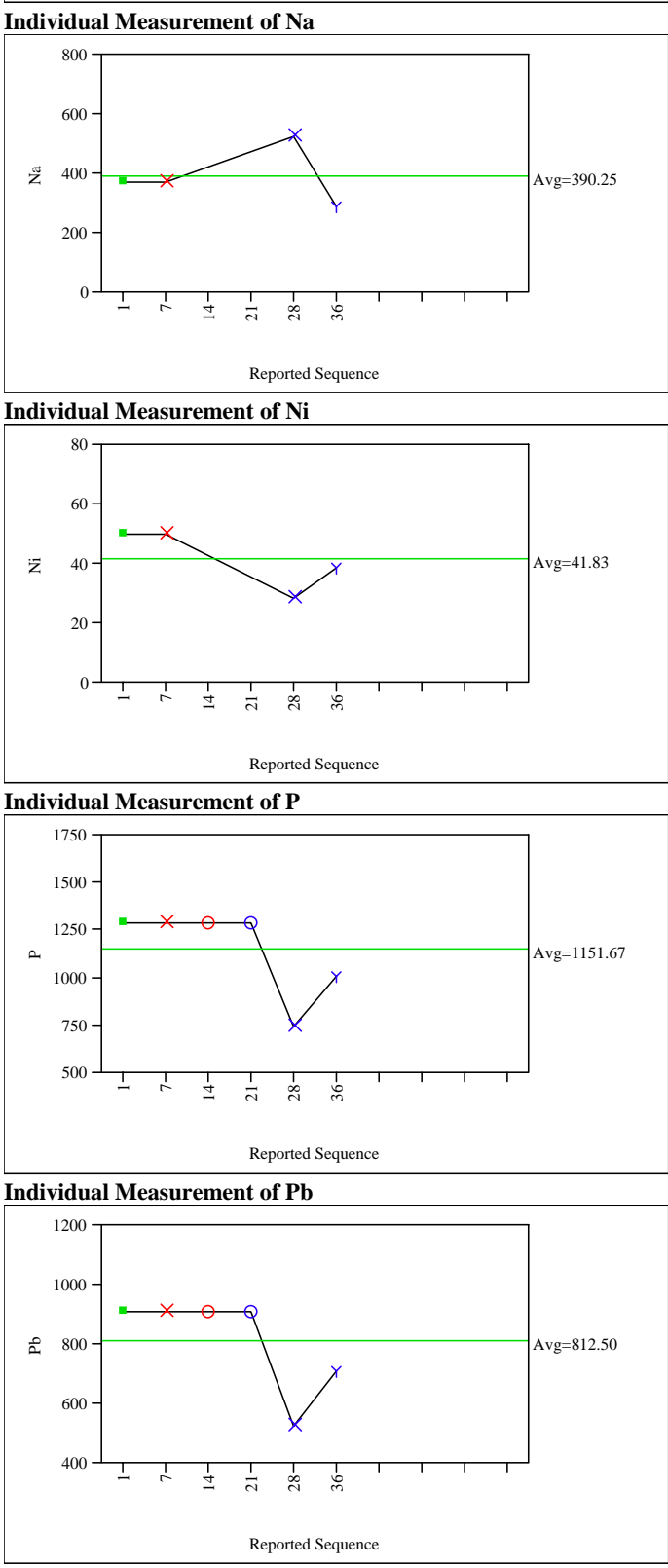
WSRC-TR-2005-00166

SRNL-RPP-2005-00029

Revision 0

\section{Exhibit A1. Elemental Measurements Plotted by Sample Type in Reported Sequence}

Type of Sample=Blank

Individual Measurement of S

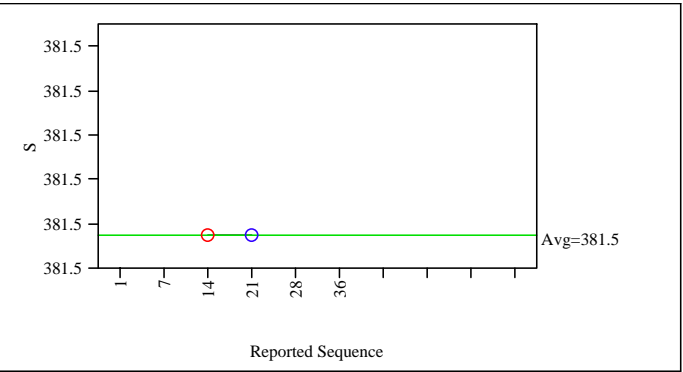

Individual Measurement of Sb

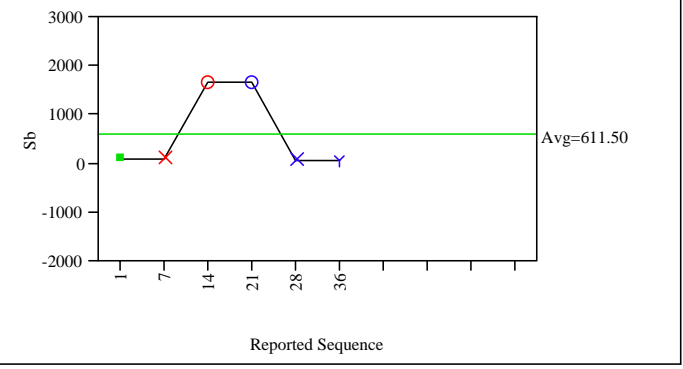

Individual Measurement of $\mathrm{Si}$

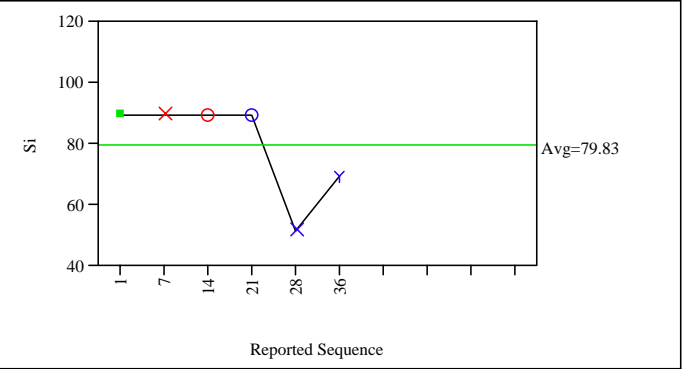

Individual Measurement of Sn

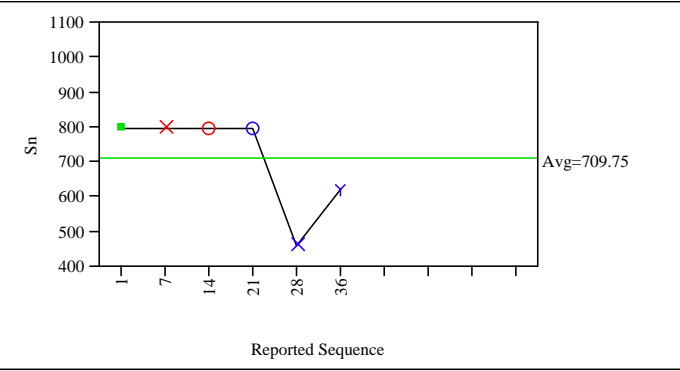

Individual Measurement of $\mathrm{Sr}$

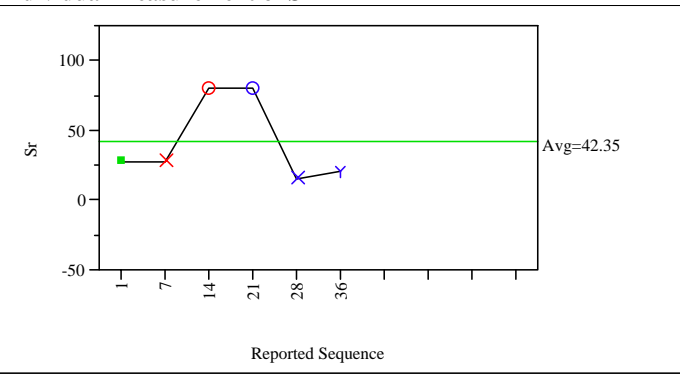

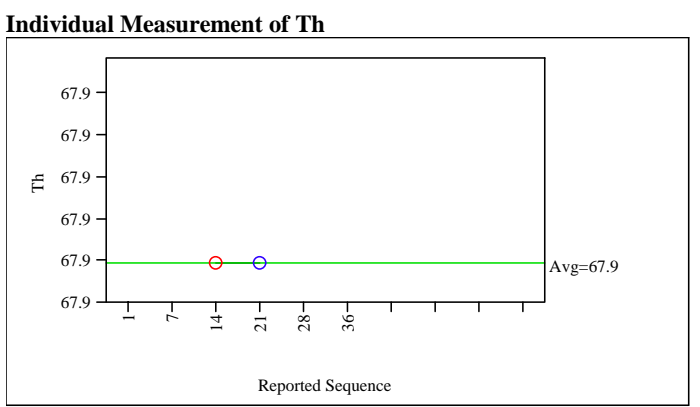

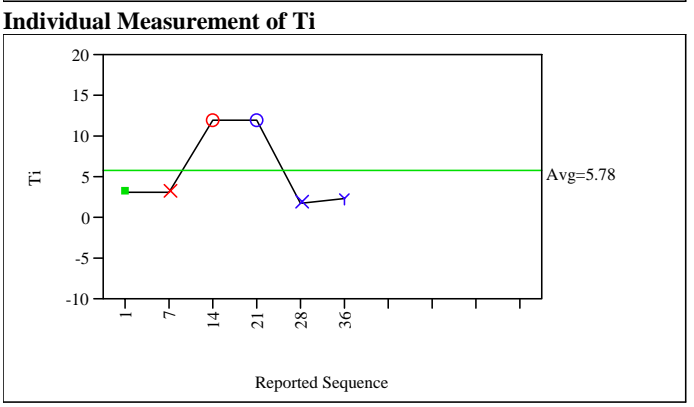

Individual Measurement of Tl
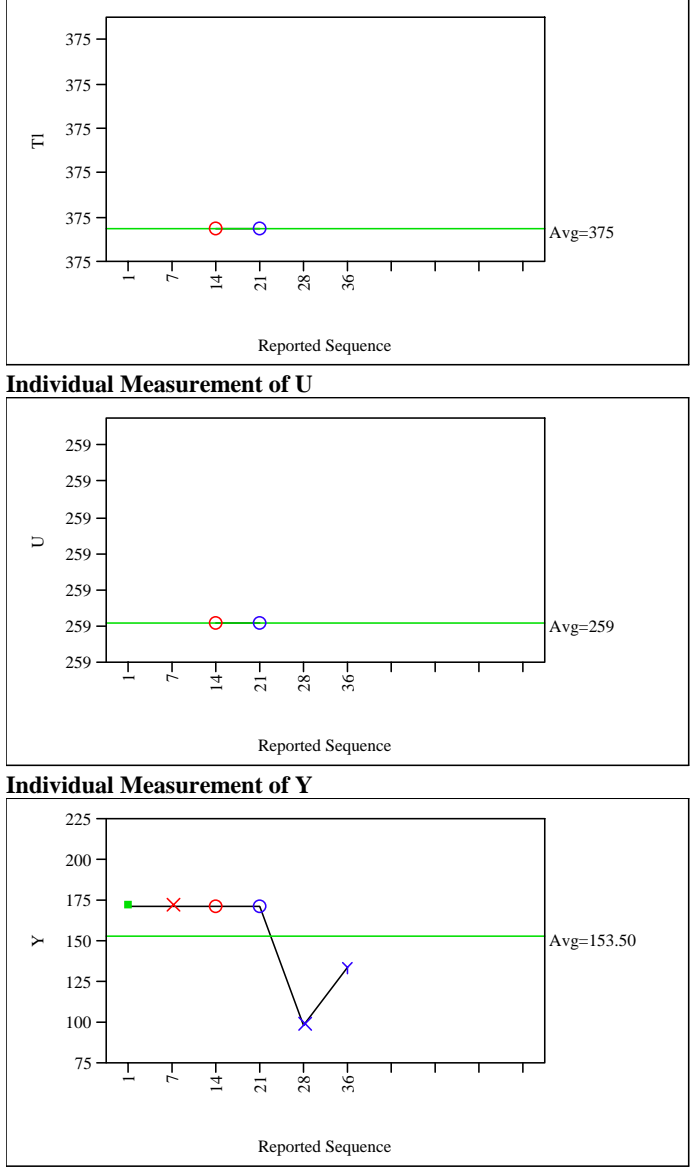
WSRC-TR-2005-00166

SRNL-RPP-2005-00029

Revision 0

\section{Exhibit A1. Elemental Measurements Plotted by Sample Type in Reported Sequence}

Type of Sample=Blank

Individual Measurement of $\mathbf{Z n}$

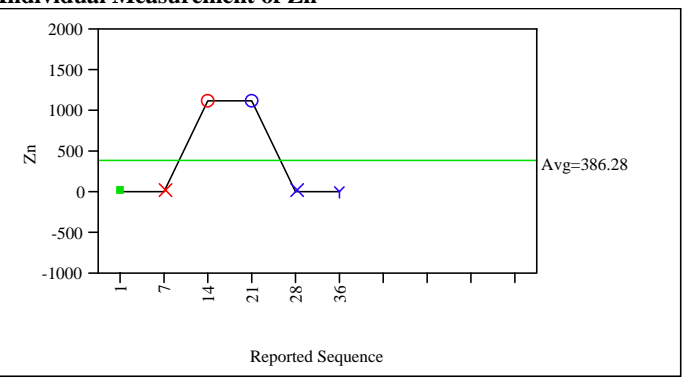

Individual Measurement of $\mathrm{Zr}$

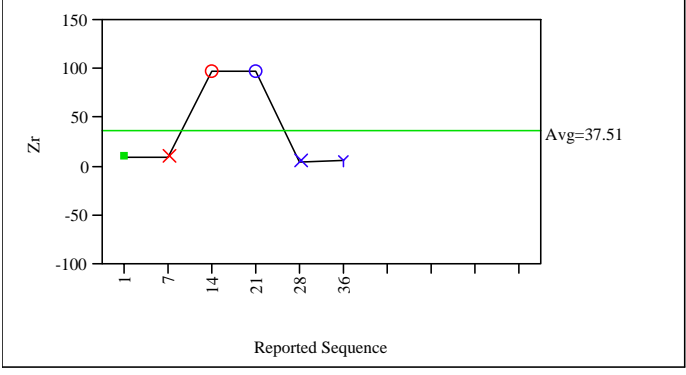

Type of Sample=LCS

Individual Measurement of Ag

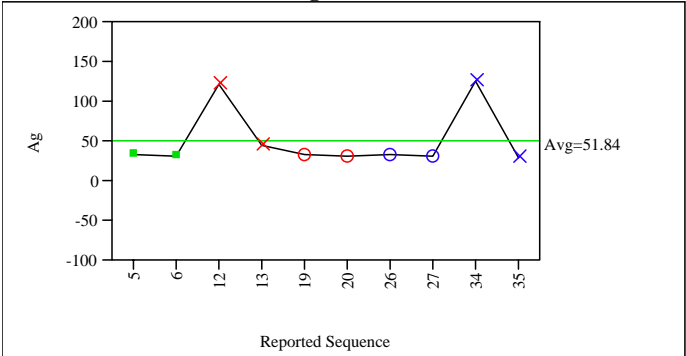

Individual Measurement of Al

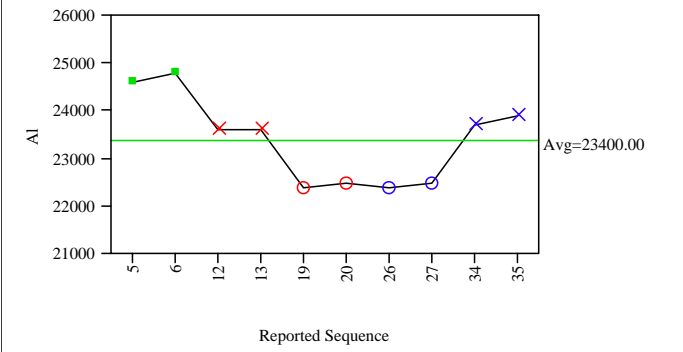

Individual Measurement of $B$

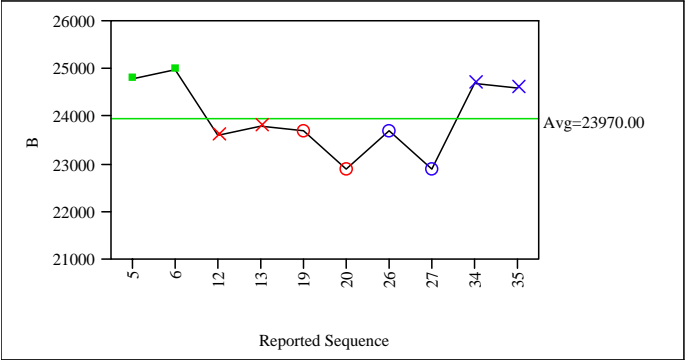

Type of Sample=LCS

Individual Measurement of Ba

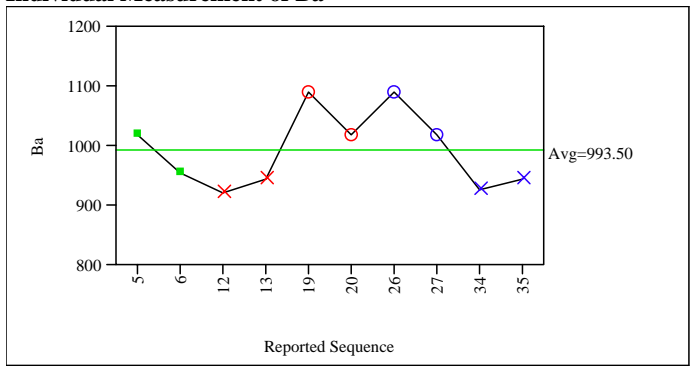

Individual Measurement of $\mathrm{Ca}$

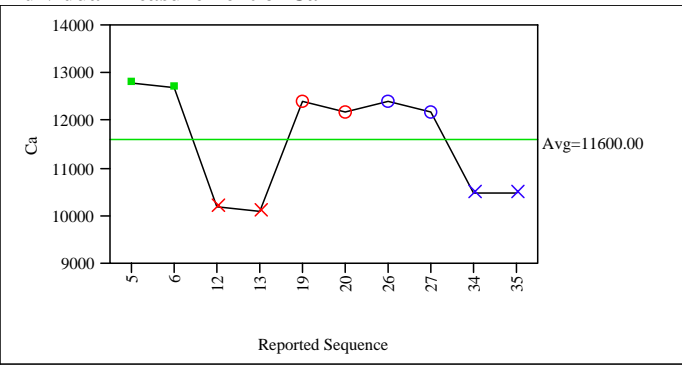

Individual Measurement of Cd

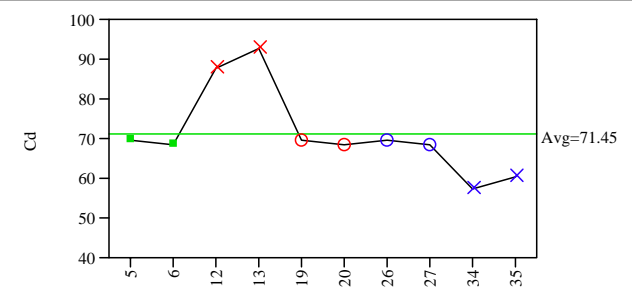

Reported Sequence

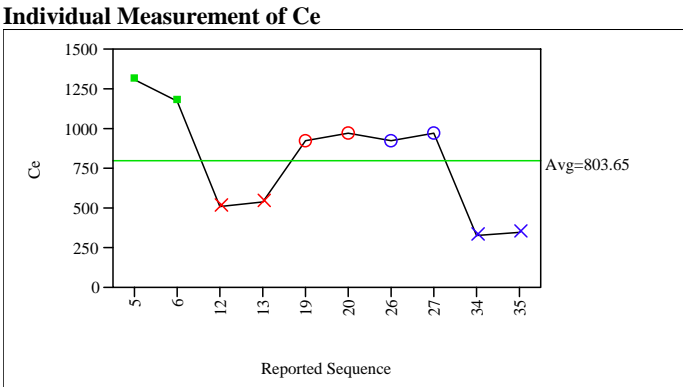

Individual Measurement of $\mathrm{Cr}$

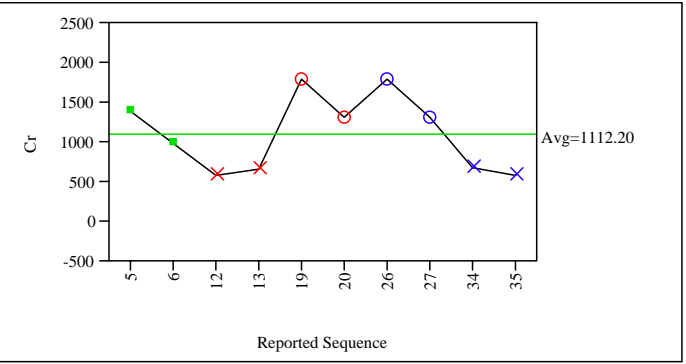


WSRC-TR-2005-00166

SRNL-RPP-2005-00029

Revision 0

\section{Exhibit A1. Elemental Measurements Plotted by Sample Type in Reported Sequence}

Type of Sample=LCS

Individual Measurement of $\mathrm{Cu}$

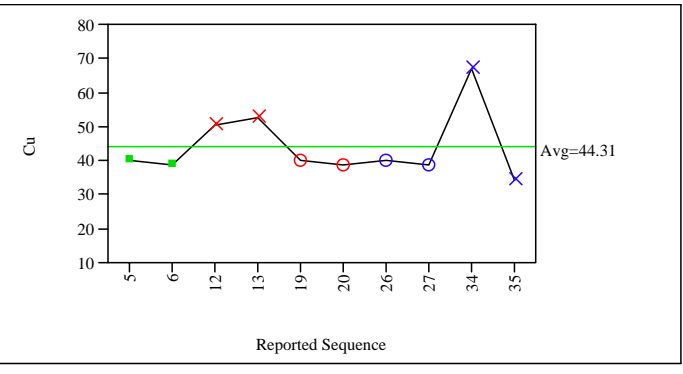

Individual Measurement of Fe

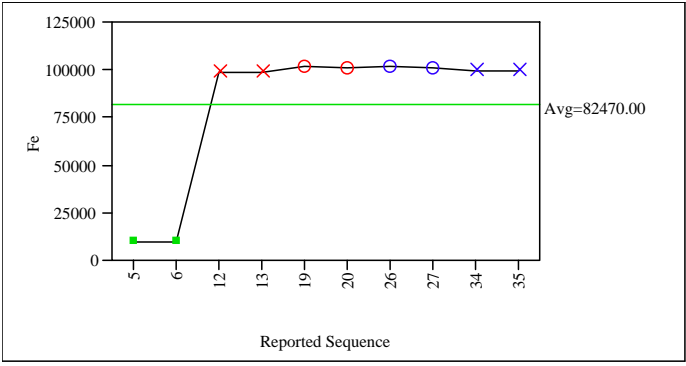

Individual Measurement of $\mathbf{K}$

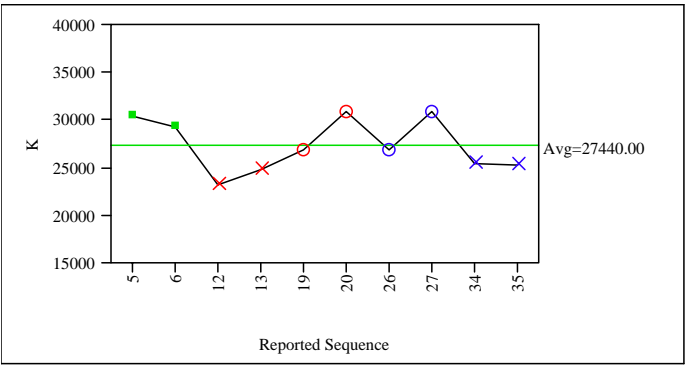

Individual Measurement of La

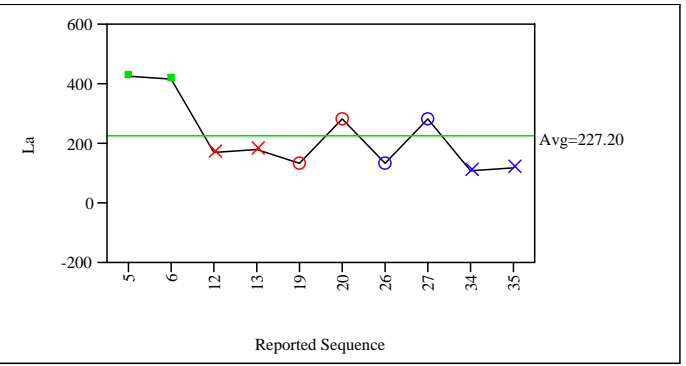

Individual Measurement of $\mathrm{Li}$

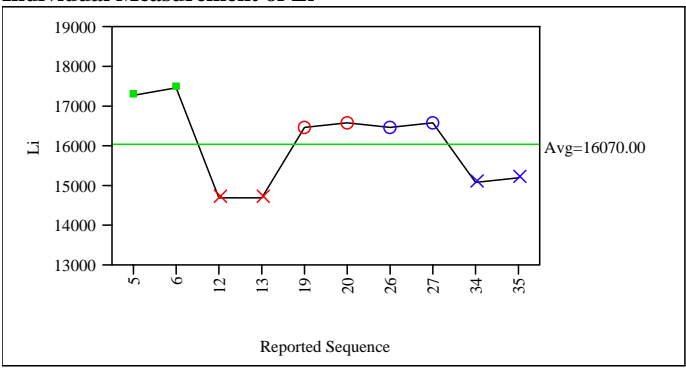

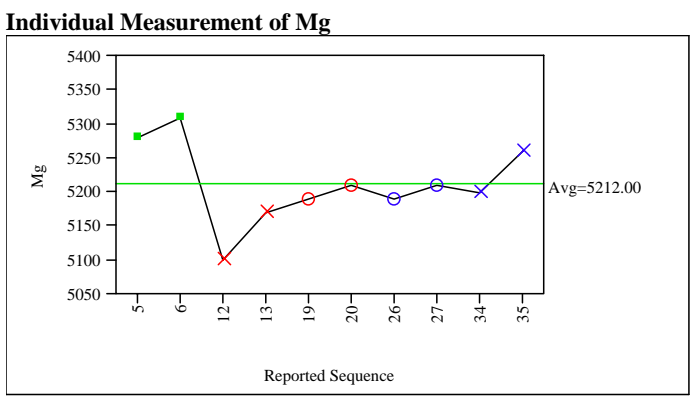

Individual Measurement of Mn

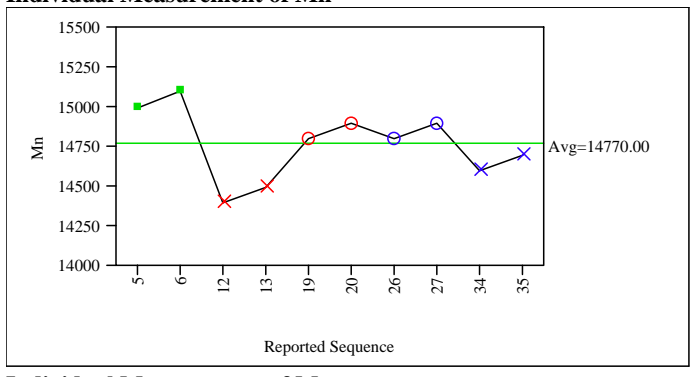

Individual Measurement of Mo

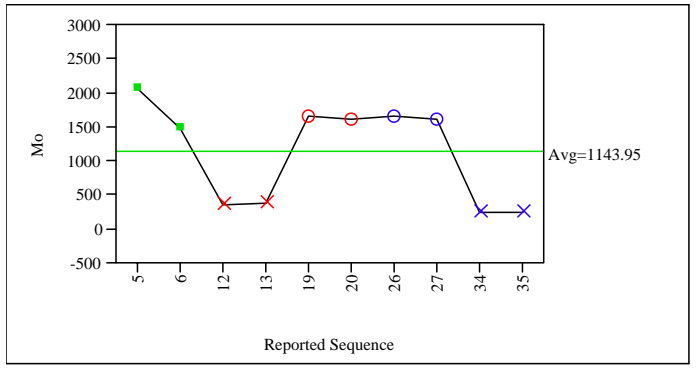

Individual Measurement of Na

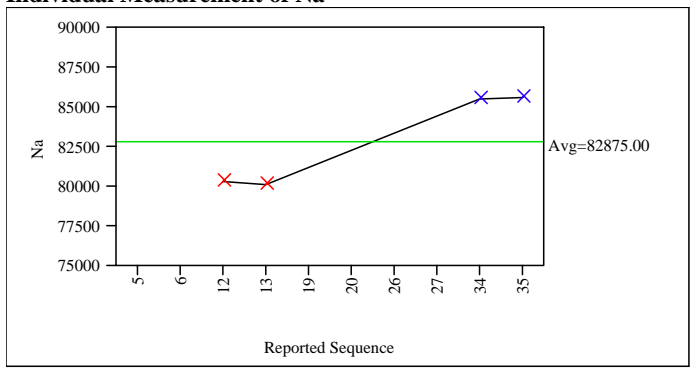

Individual Measurement of $\mathrm{Ni}$

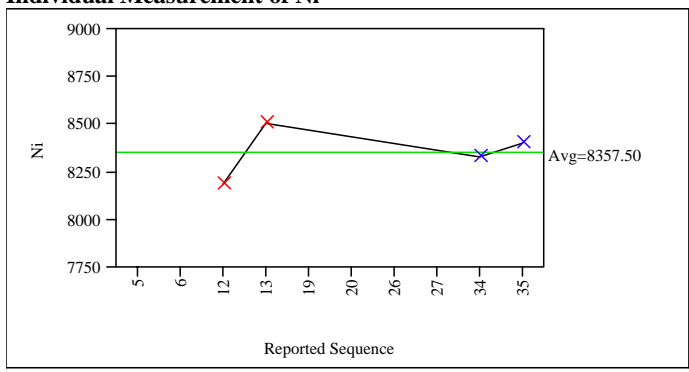


WSRC-TR-2005-00166

SRNL-RPP-2005-00029

Revision 0

\section{Exhibit A1. Elemental Measurements Plotted by Sample Type in Reported Sequence}

Type of Sample=LCS

Individual Measurement of $P$

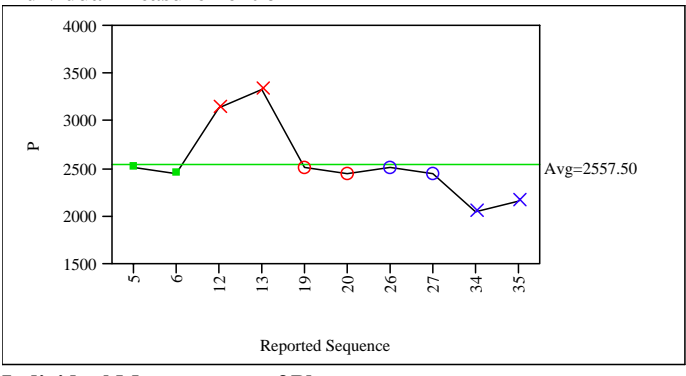

Individual Measurement of Pb

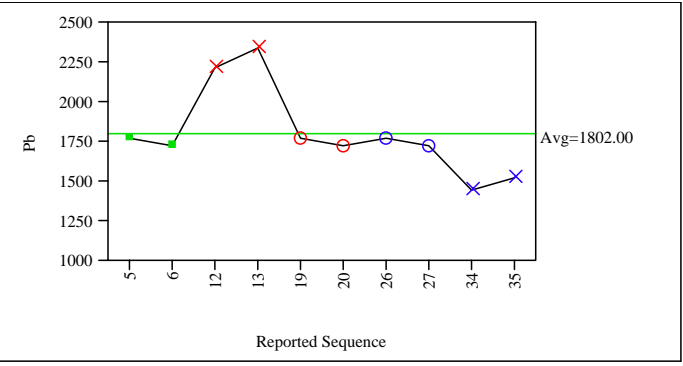

Individual Measurement of S

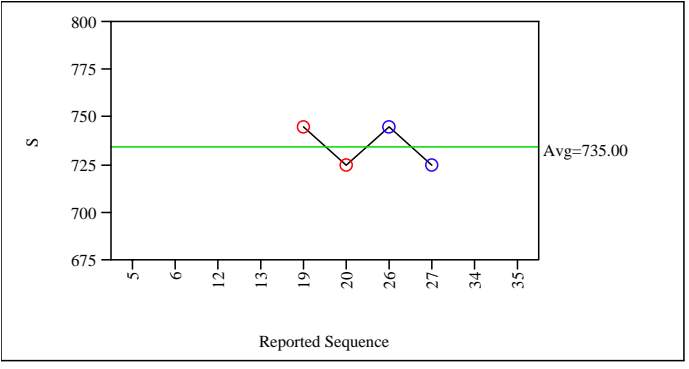

Individual Measurement of Sb

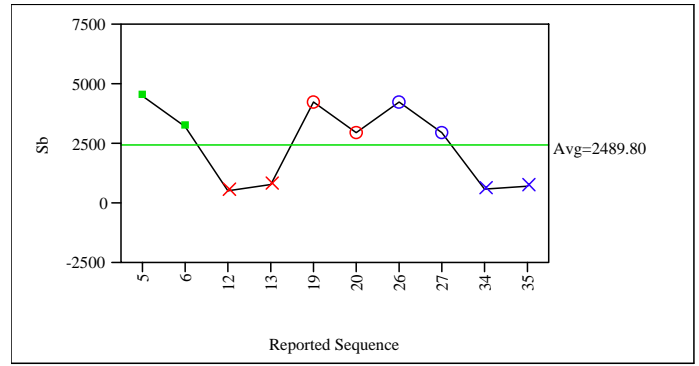

Individual Measurement of Si

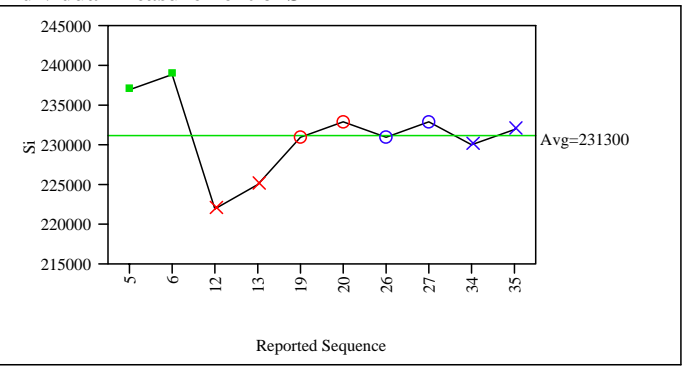

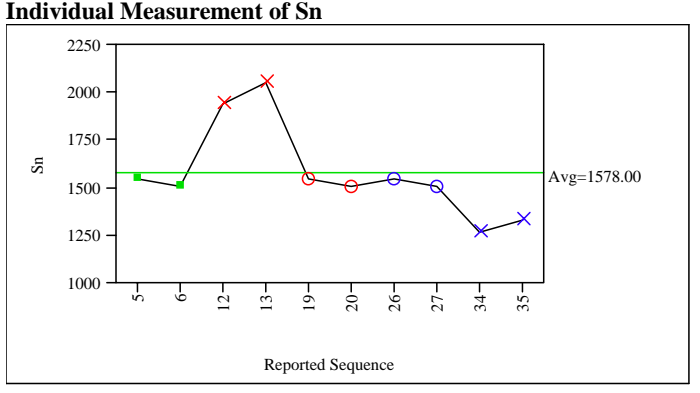

Individual Measurement of Sr

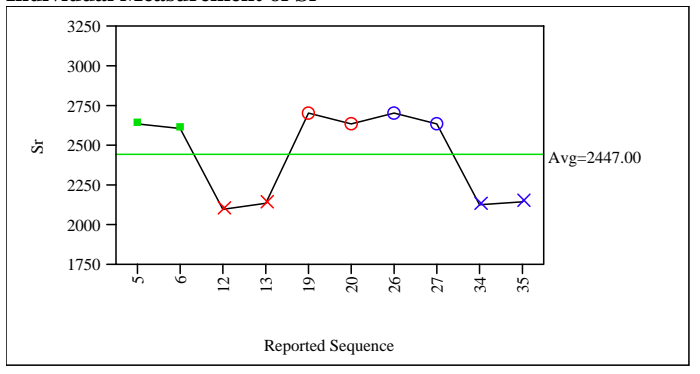

Individual Measurement of Th

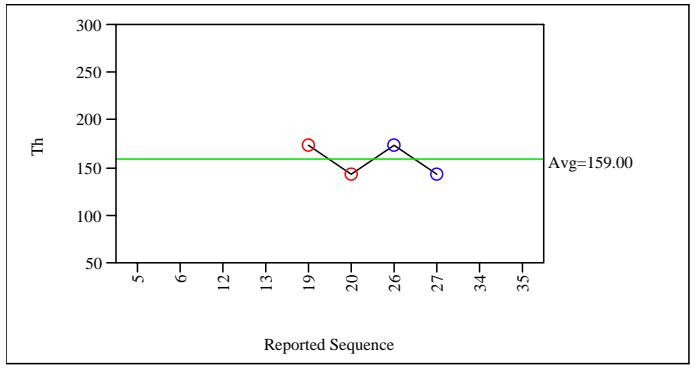

Individual Measurement of $\mathrm{T}$

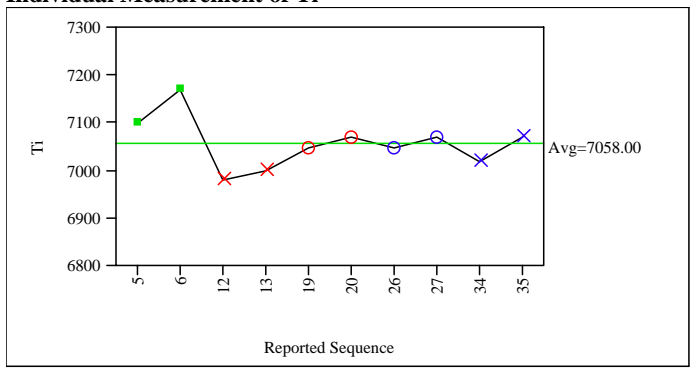

Individual Measurement of Tl

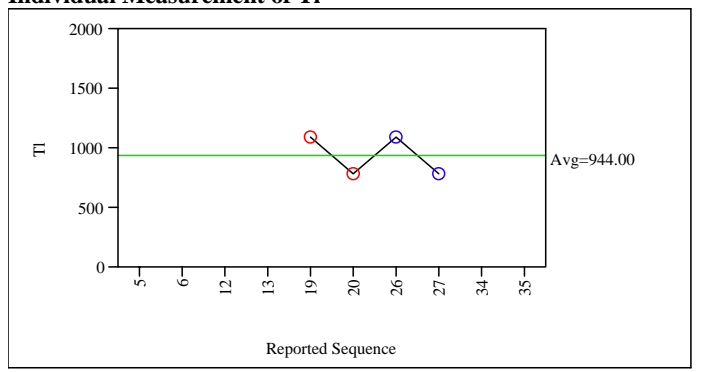


WSRC-TR-2005-00166

SRNL-RPP-2005-00029

Revision 0

\section{Exhibit A1. Elemental Measurements Plotted by Sample Type in Reported Sequence}

Type of Sample=LCS

Individual Measurement of $\mathbf{U}$

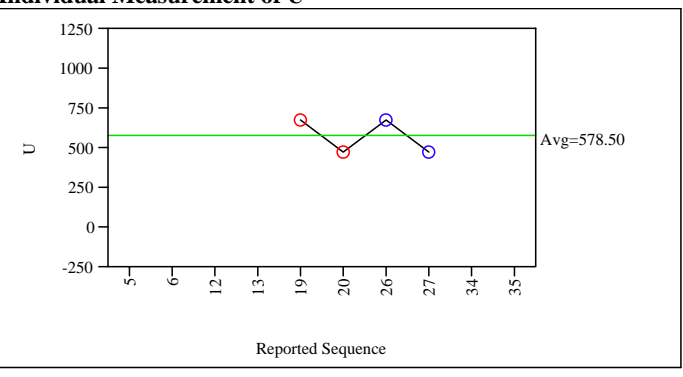

Individual Measurement of Y

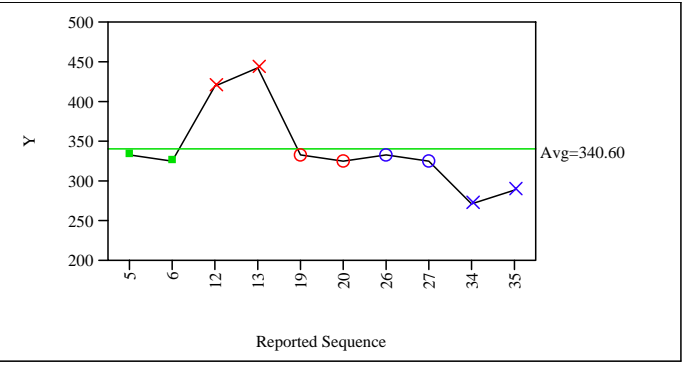

Individual Measurement of $\mathrm{Zn}$

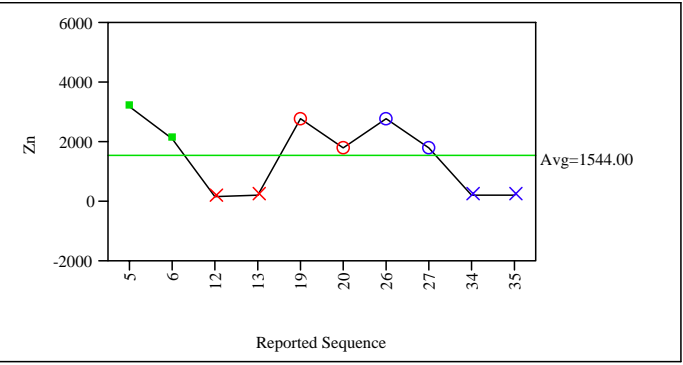

Individual Measurement of $\mathrm{Zr}$

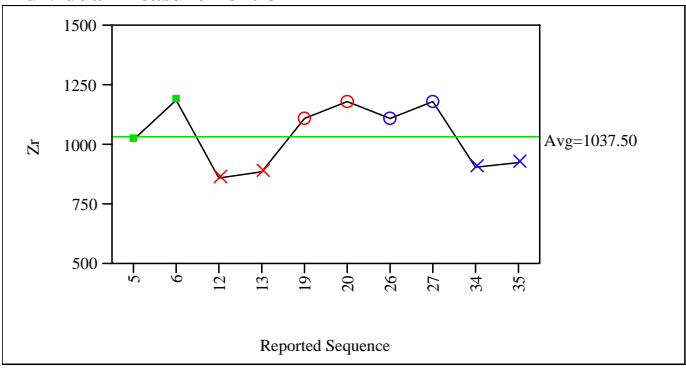

Type of Sample=Trial

Individual Measurement of Ag

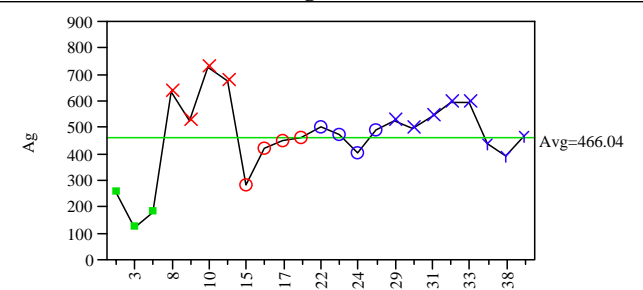

Reported Sequence
Type of Sample= $=$ Trial

Individual Measurement of Al

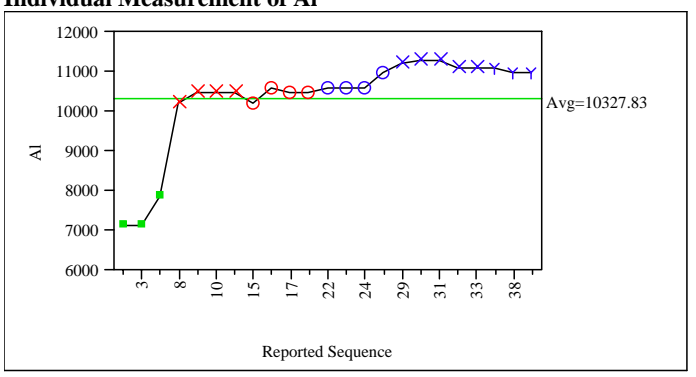

Individual Measurement of B

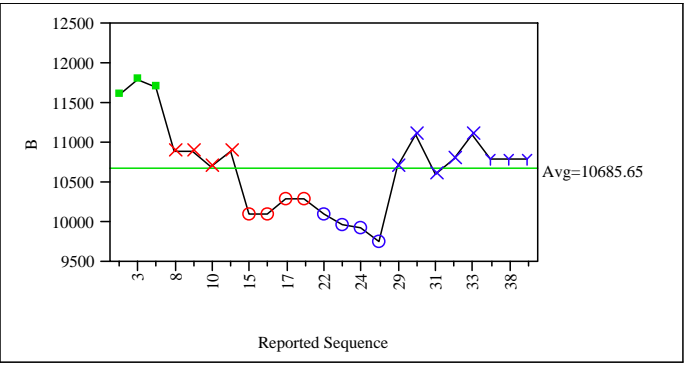

Individual Measurement of Ba

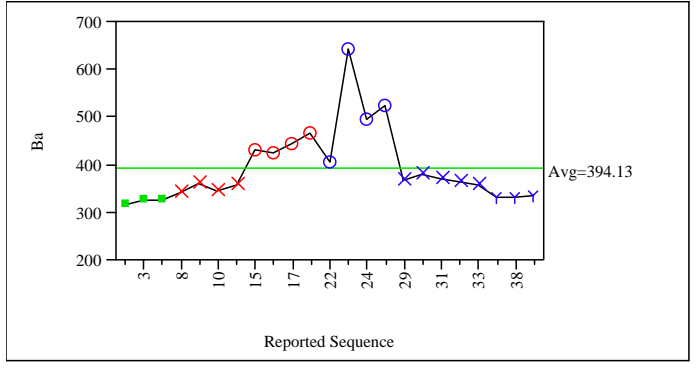

Individual Measurement of $\mathrm{Ca}$

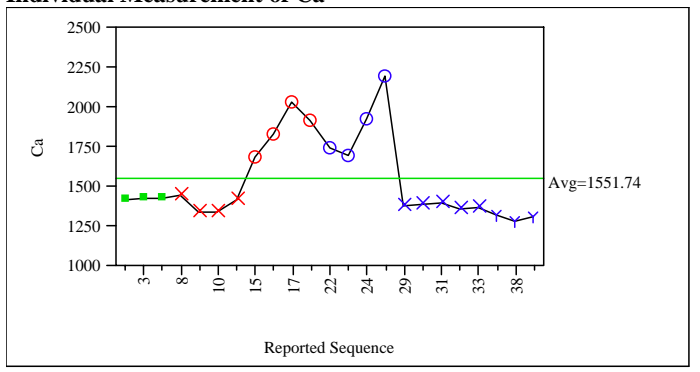

Individual Measurement of Cd

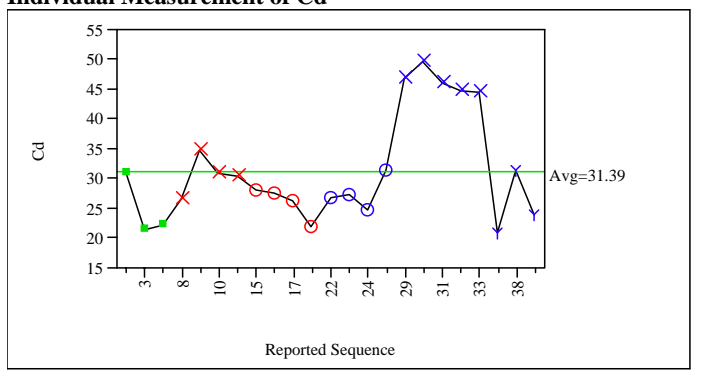


WSRC-TR-2005-00166

SRNL-RPP-2005-00029

Revision 0

\section{Exhibit A1. Elemental Measurements Plotted by Sample Type in Reported Sequence}

Type of Sample=Trial

Individual Measurement of $\mathrm{Ce}$

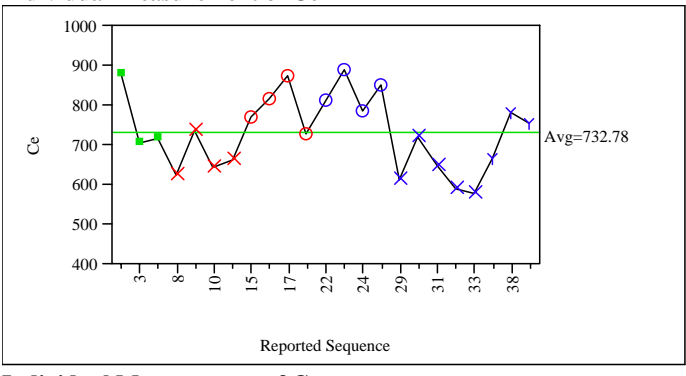

Individual Measurement of $\mathrm{Cr}$

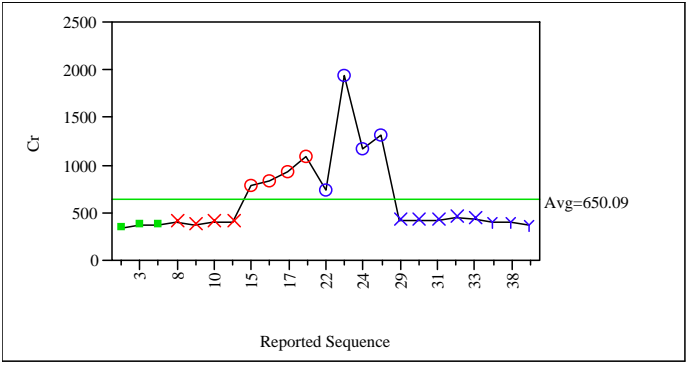

Individual Measurement of $\mathrm{Cu}$

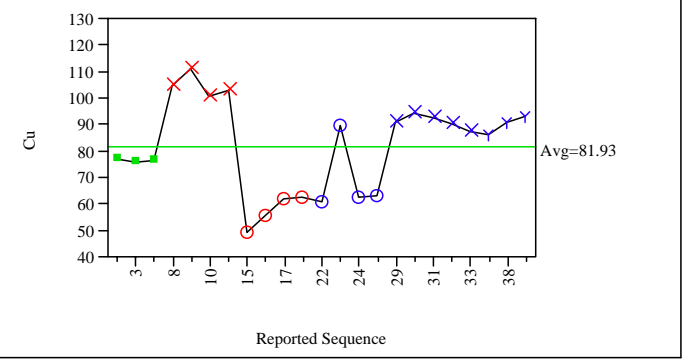

Individual Measurement of $\mathrm{Fe}$

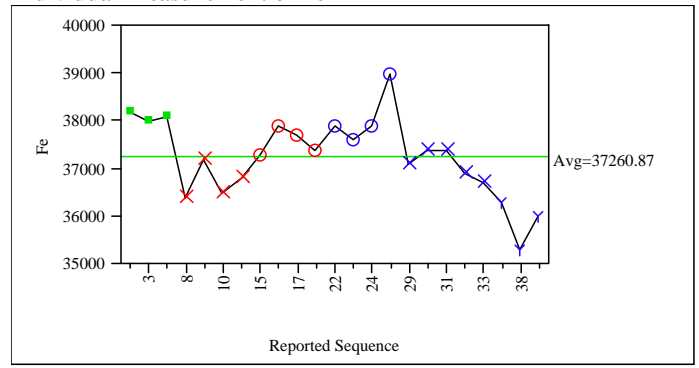

Individual Measurement of $\mathbf{K}$

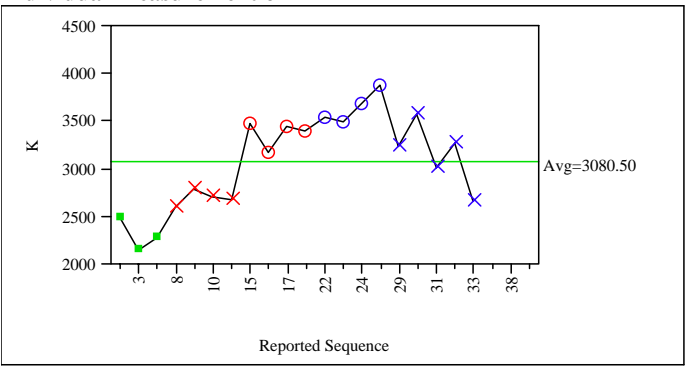

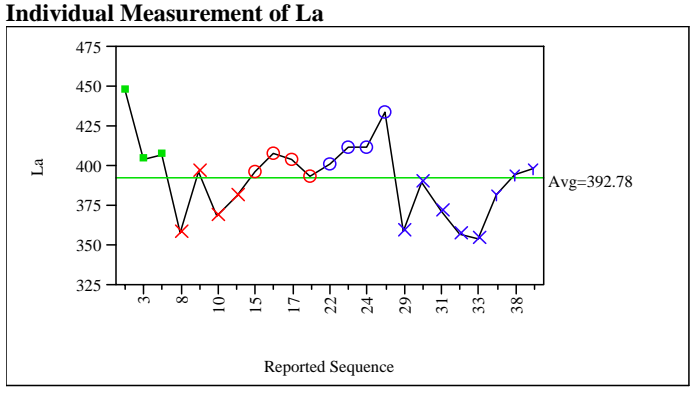

Individual Measurement of $\mathrm{Li}$

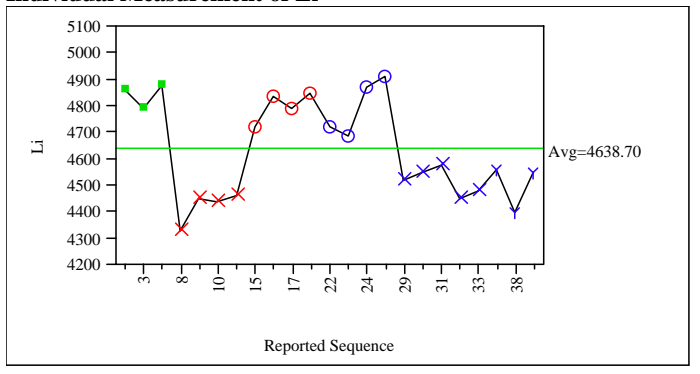

Individual Measurement of $\mathrm{Mg}$

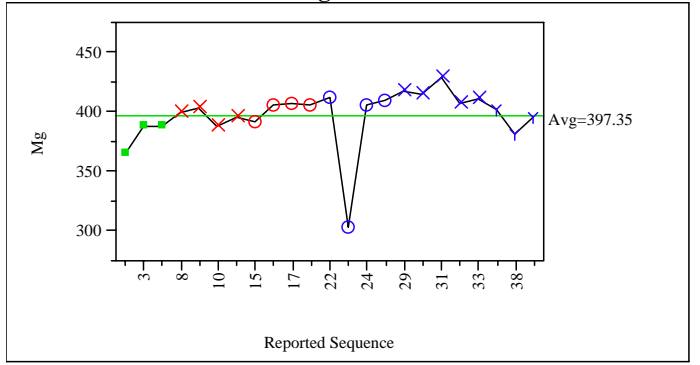

Individual Measurement of Mn

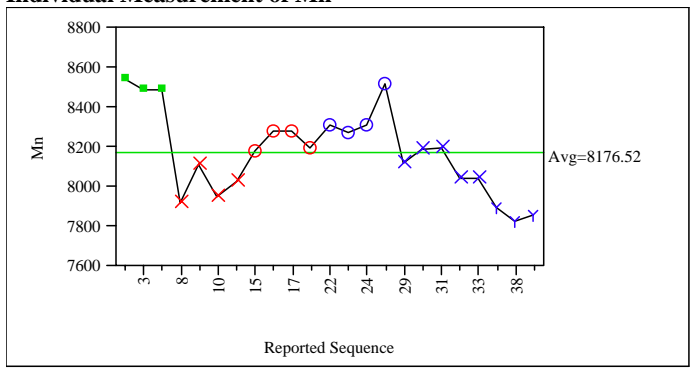

Individual Measurement of Mo

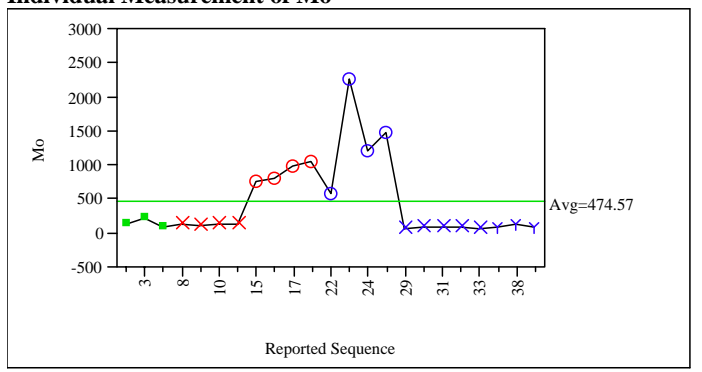


WSRC-TR-2005-00166

SRNL-RPP-2005-00029

Revision 0

\section{Exhibit A1. Elemental Measurements Plotted by Sample Type in Reported Sequence}

Type of Sample=Trial

Individual Measurement of $\mathrm{Na}$

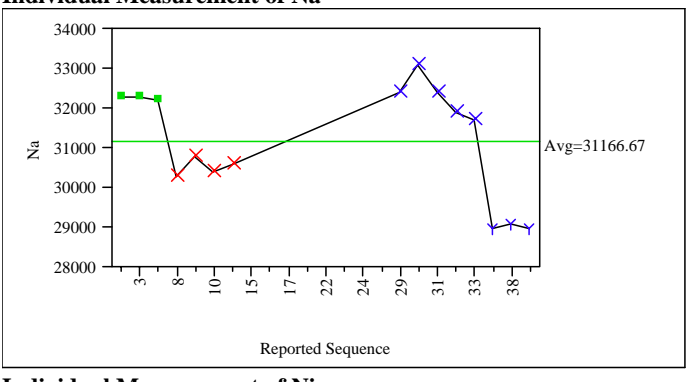

Individual Measurement of $\mathrm{Ni}$

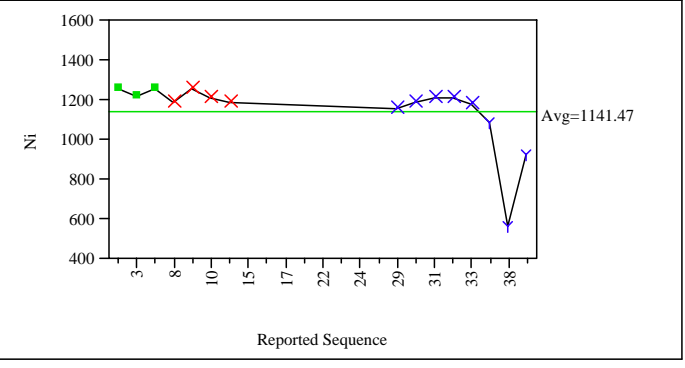

Individual Measurement of $\mathbf{P}$

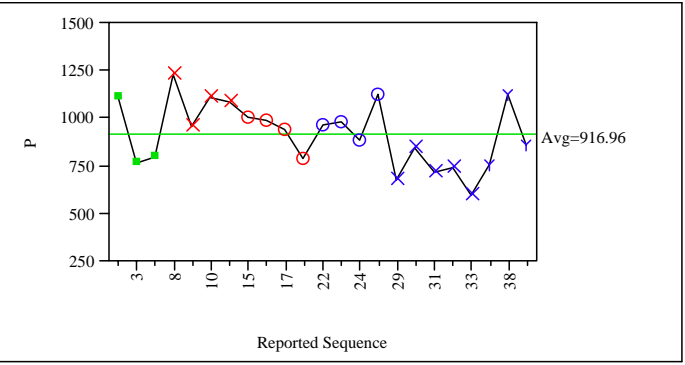

Individual Measurement of $\mathbf{P b}$

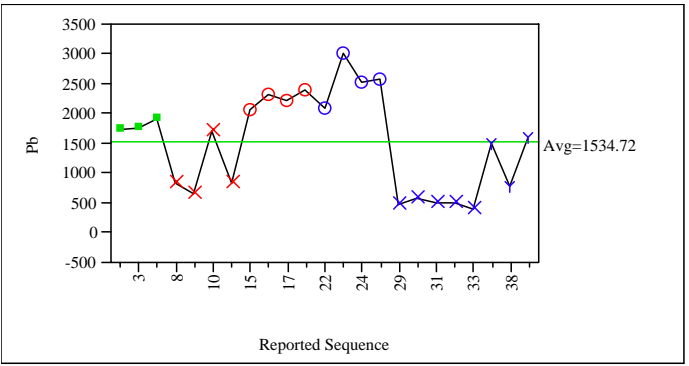

Individual Measurement of S

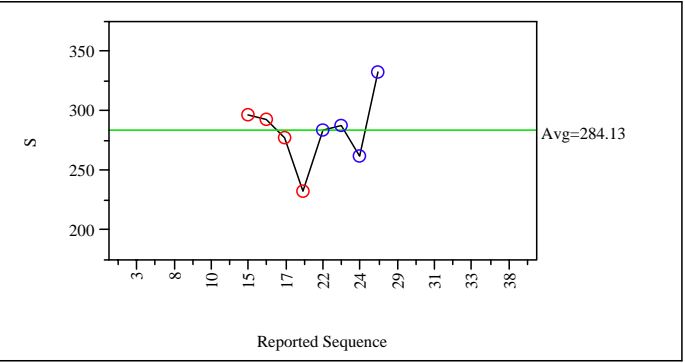

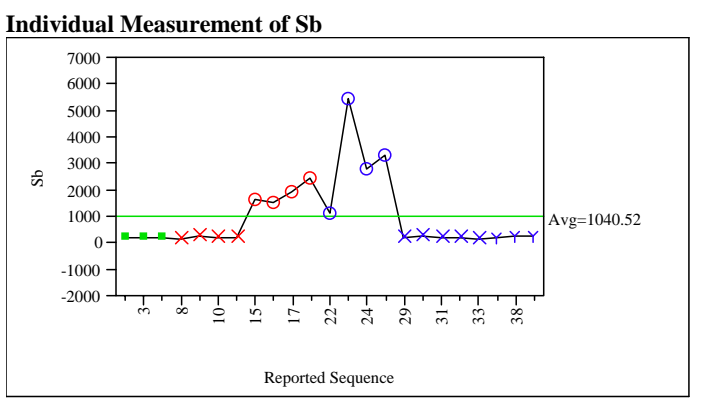

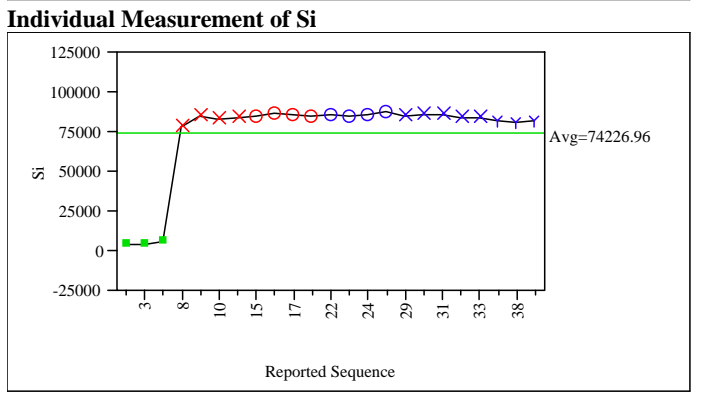

Individual Measurement of Sn

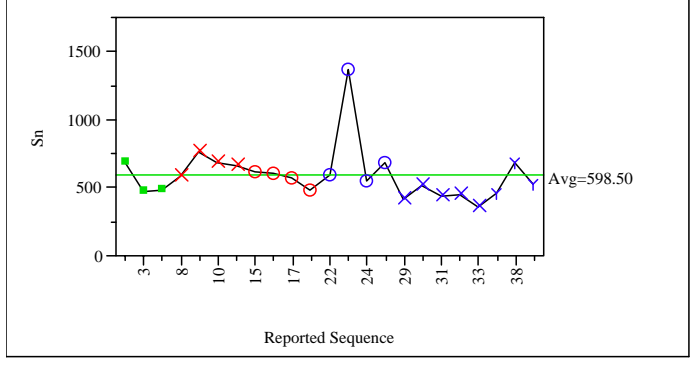

Individual Measurement of Sr

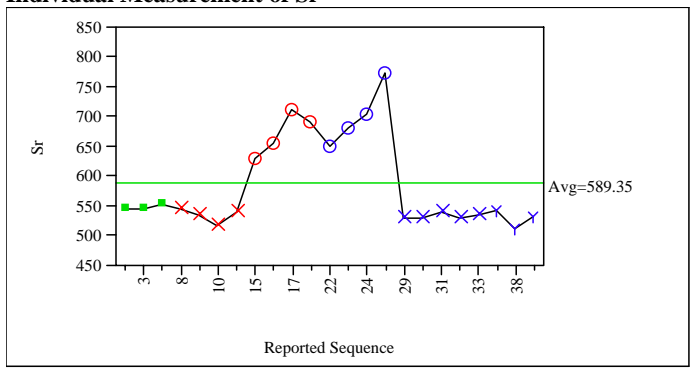

Individual Measurement of Th

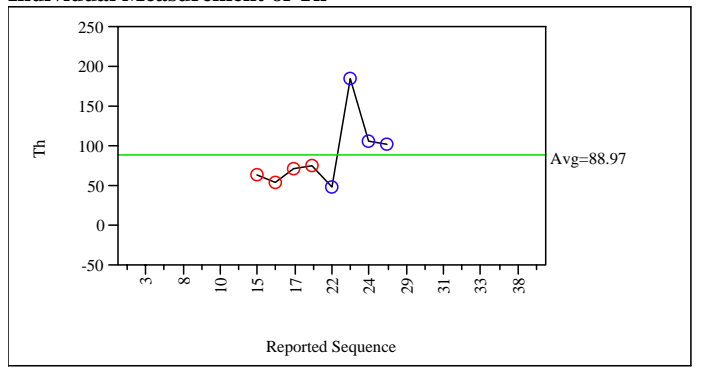


WSRC-TR-2005-00166

SRNL-RPP-2005-00029

Revision 0

\section{Exhibit A1. Elemental Measurements Plotted by Sample Type in Reported Sequence}

Type of Sample=Trial

Individual Measurement of $\mathbf{T i}$

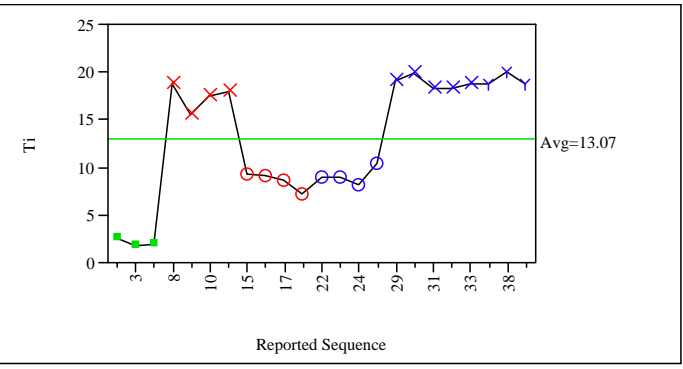

Individual Measurement of Tl

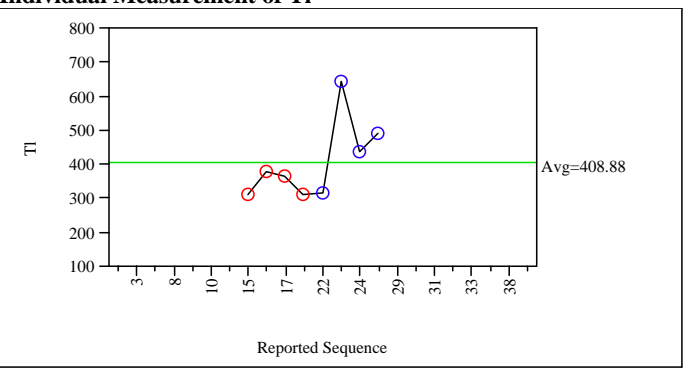

Individual Measurement of U

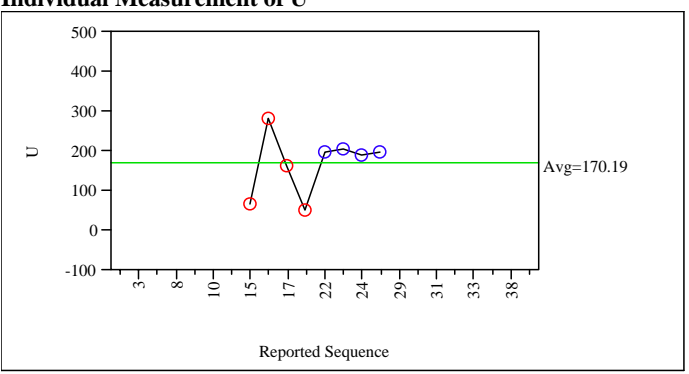

Individual Measurement of Y

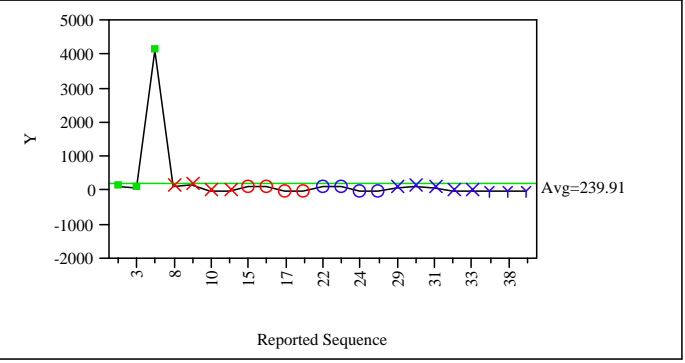

Individual Measurement of Zn

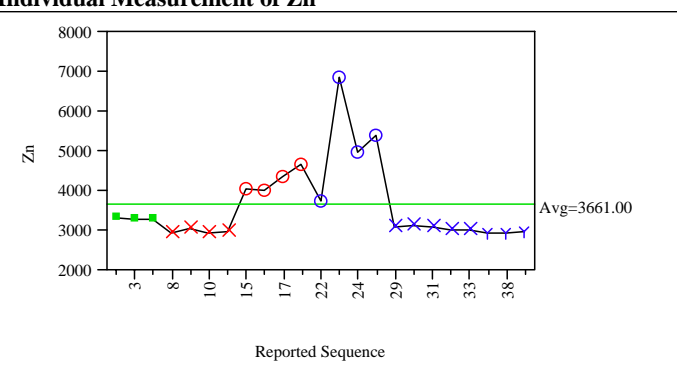

Individual Measurement of $\mathrm{Zr}$

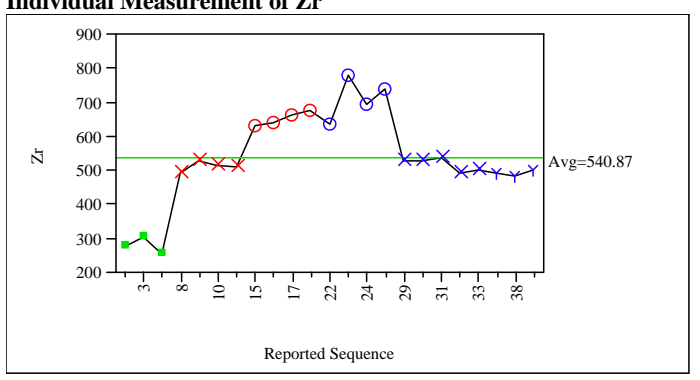


WSRC-TR-2005-00166

SRNL-RPP-2005-00029

Revision 0

Exhibit A2. Statistical Comparisons of Preparation/Crucible Measurements by Element by Type of Sample

Type of Sample=Blank

Oneway Analysis of Ag By Simulant/Preparation/Crucible

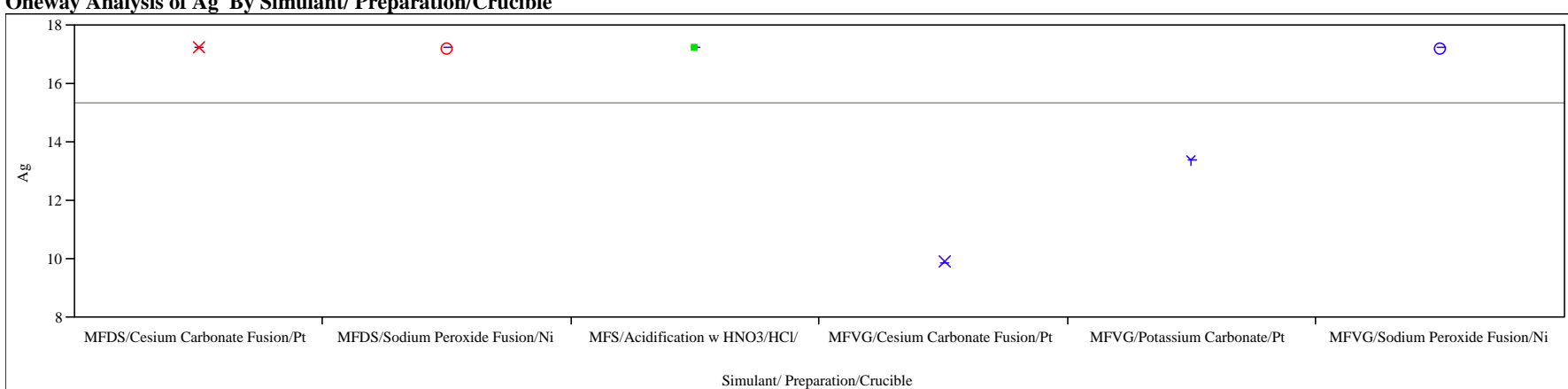

Oneway Anova

Summary of Fit

Rsquare

Adj Rsquare

Root Mean Square Error

Mean of Response

Observations (or Sum Wgts)

Analysis of Variance

Source

Simulant/ Preparation/Crucible

Error

Means for Oneway Anova

Means

Level

MFDS/Cesium Carbonate Fusion/Pt

MFS/Aodidin Peroxide Fusion/Ni

MFVG/Cesim Ca WHOB/HCl/

MFVG/Cesium Carbonate Fusion/Pt

MFVG/Sodium Peabose

Ste

Preparation/Cruc

Means and Std Deviations

Level

MFDS/Cesium Carbonate Fusion/Pt

TFS/Sodin Peroxide Fusion/Ni

MFVG/Cicaton w HNO3/HCl/

MFVG/Cosiun Catbonate Fusion

MFVG/Soliom Peroxide Fusior

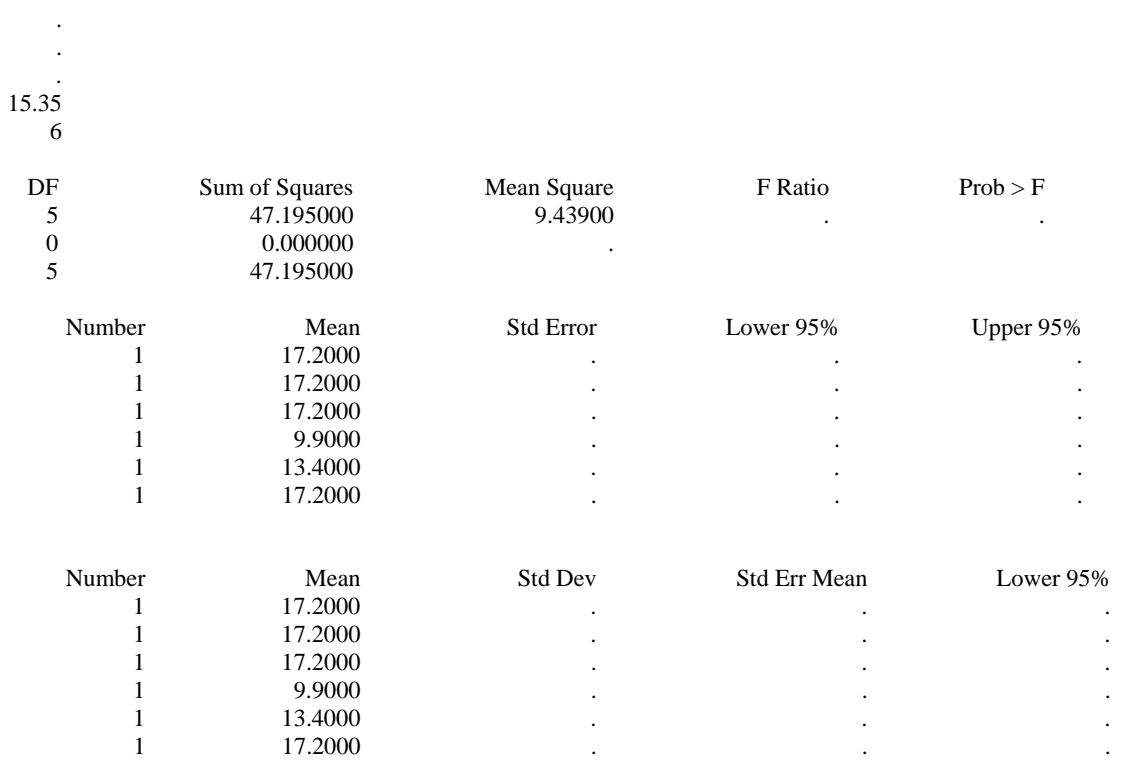

Upper 95\% 
WSRC-TR-2005-00166

SRNL-RPP-2005-00029

Revision 0

Exhibit A2. Statistical Comparisons of Preparation/Crucible Measurements by Element by Type of Sample

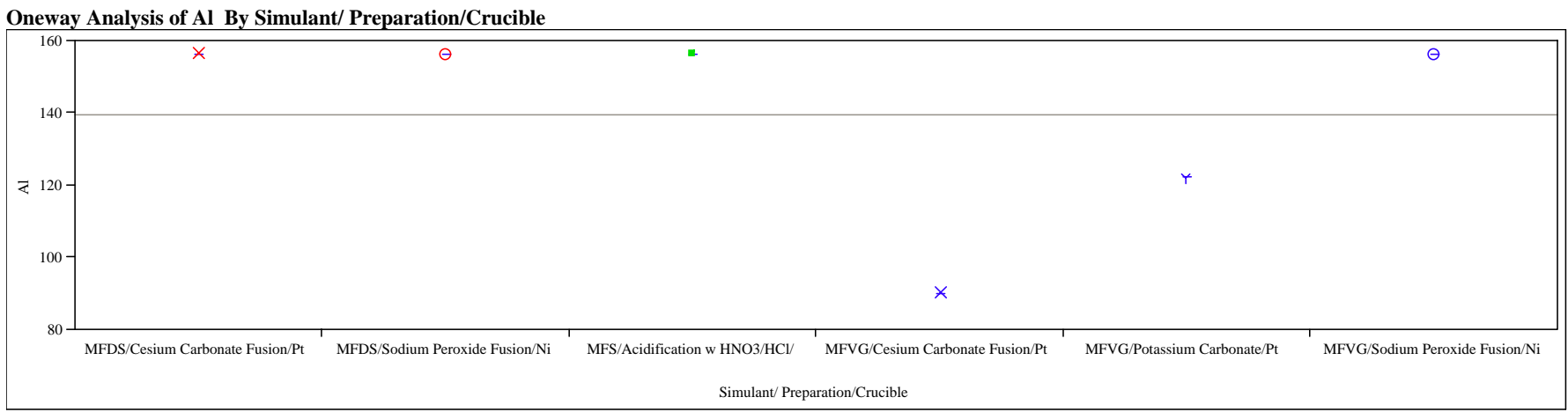

Oneway Anova

Summary of Fit

Rsquare

Adj Rsquare

Root Mean Square Error

Mean of Response

Observations (or Sum Wgts)

Analysis of

Source

Simulant/ Preparation/Crucible

Error

C. Total

Means

MFDS/Cesium Carbonate Fusion/Pt

/

MFVG/Cesium Carbonate Fusion/Pt

MFVG/Potassium Carbonate/Pt

MFVG/Sodium Peroxide Fusion/Ni

Std Error uses a pooled estimate of error variance

Means and Std Deviations

Level

MFDS/Cesium Carbonate Fusion/Pt

MFDS/Sodium Peroxide Fusion/N

MFS/Acidification w HNO3/HCl

MFVG/Cesium Carbonate Fusion/Pt

MFVG/Potassium Carbonate/Pt

MFVG/Sodium Peroxide Fusion/Ni

\begin{tabular}{|c|c|c|c|c|}
\hline DF & Sum of Squares & Mean Square & F Ratio & Prob $>$ F \\
\hline 5 & 3912.3333 & 782.467 & & . \\
\hline 0 & 0.0000 & & & \\
\hline 5 & 3912.3333 & & & \\
\hline Number & Mean & Std Error & Lower 95\% & Upper 95\% \\
\hline 1 & 156.500 & & . & . \\
\hline 1 & 156.500 & . & . & . \\
\hline 1 & 156.500 & . & . & . \\
\hline 1 & 90.000 & . & . & . \\
\hline 1 & 122.000 & . & . & . \\
\hline 1 & 156.500 & . & . & . \\
\hline Number & Mean & Std Dev & Std Err Mean & Lower 95\% \\
\hline 1 & 156.500 & & . & \\
\hline 1 & 156.500 & & . & \\
\hline 1 & 156.500 & . & . & \\
\hline 1 & 90.000 & . & & \\
\hline 1 & 122.000 & & . & \\
\hline 1 & 156.500 & . & . & \\
\hline
\end{tabular}

Upper 95\% 
WSRC-TR-2005-00166

SRNL-RPP-2005-00029

Revision 0

Exhibit A2. Statistical Comparisons of Preparation/Crucible Measurements by Element by Type of Sample

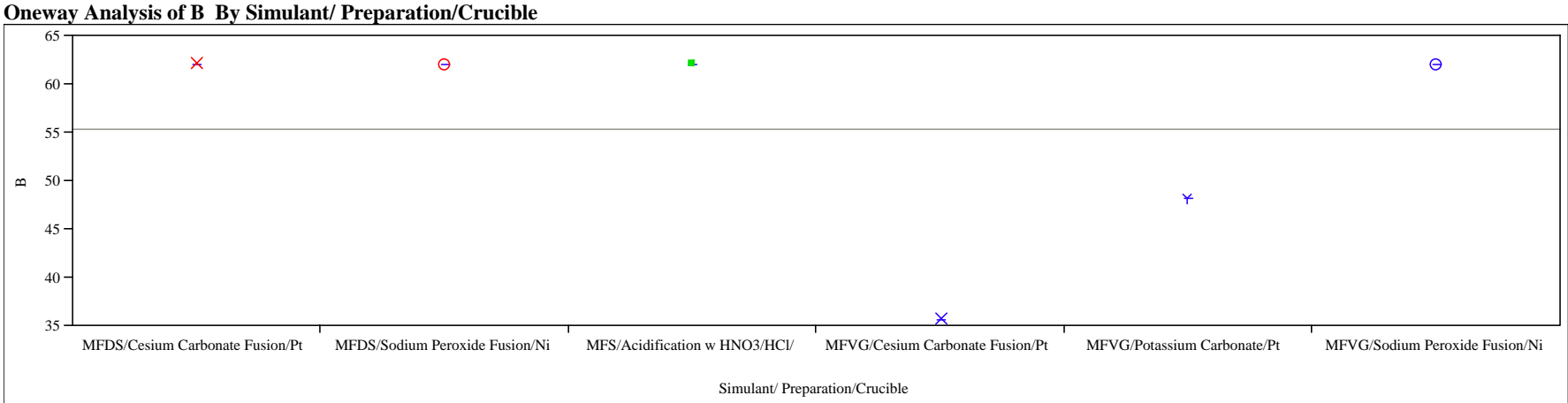

Oneway Anova

Summary of Fit

Rsquare

Adj Rsquare

Root Mean Square Error

Mean of Response

Observations (or Sum Wgts)

Analysis of

Source

Simulant/ Preparation/Crucible

Error

C. Total

Level

Preparation/Crucible

MFDS/Cesium Carbonate Fusion/Pt

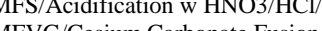

MFVG/Potassium Carbona/Pt

MFVG/Sodium Parox Fusion

of error variance

Means and

MFDel

MFDS/Cesium Carbonate Fusion/Pt

MFDS/Sodium Peroxide Fusion/Ni

MFS/Acidification w HNO3/HCl

MFVG/Cesium Carbonate Fusion/Pt

MFVG/Potassium Carbonate/Pt

MFVG/Sodium Peroxide Fusion/Ni

\begin{tabular}{|c|c|c|c|c|}
\hline DF & Sum of Squares & Mean Square & F Ratio & Prob $>$ F \\
\hline 5 & 615.38333 & 123.077 & & \\
\hline 0 & 0.00000 & & & \\
\hline 5 & 615.38333 & & & \\
\hline Number & Mean & Std Error & Lower 95\% & Upper 95\% \\
\hline 1 & 62.0000 & & . & . \\
\hline 1 & 62.0000 & & . & . \\
\hline 1 & 62.0000 & & . & . \\
\hline 1 & 35.6500 & . & . & . \\
\hline 1 & 48.2500 & & . & . \\
\hline 1 & 62.0000 & . & . & . \\
\hline Number & Mean & Std Dev & Std Err Mean & Lower 95\% \\
\hline 1 & 62.0000 & & 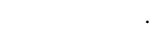 & \\
\hline 1 & 62.0000 & . & . & \\
\hline 1 & 62.0000 & & . & \\
\hline 1 & 35.6500 & . & . & \\
\hline 1 & 48.2500 & & . & \\
\hline 1 & 62.0000 & & . & \\
\hline
\end{tabular}

Upper 95\% 
WSRC-TR-2005-00166

SRNL-RPP-2005-00029

Revision 0

Exhibit A2. Statistical Comparisons of Preparation/Crucible Measurements by Element by Type of Sample

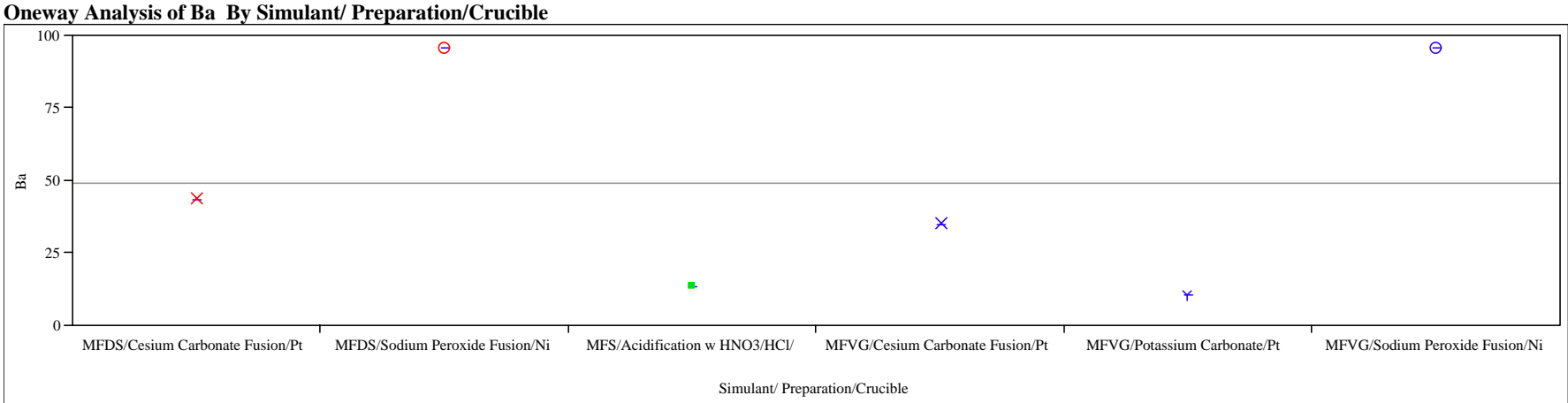

Oneway Anova

Summary of Fit

Rsquare

Adj Rsquare

Root Mean Square Erro

Mean of Response

Observations (or Sum Wgts)

Analysis of

Source

Simulant/ Preparation/Crucible

Error

C. Total

Means

MFDS/Cesium Carbonate Fusion/Pt

A

MFVG/Cesium Carbonate Fusion/Pt

MFVG/Potassium Carbonate/Pt

MFVG/Sodium Peroxide Fusion/Ni

Std Error uses a pooled estimate of error variance

Means and Std Deviations

Level

MFDS/Cesium Carbonate Fusion/Pt

MFDS/Sodium Peroxide Fusion/N

MFS/Acidification w HNO3/HCl

MFVG/Cesium Carbonate Fusion/Pt

MFVG/Potassium Carbonate/Pt

MFVG/Sodium Peroxide Fusion/Ni

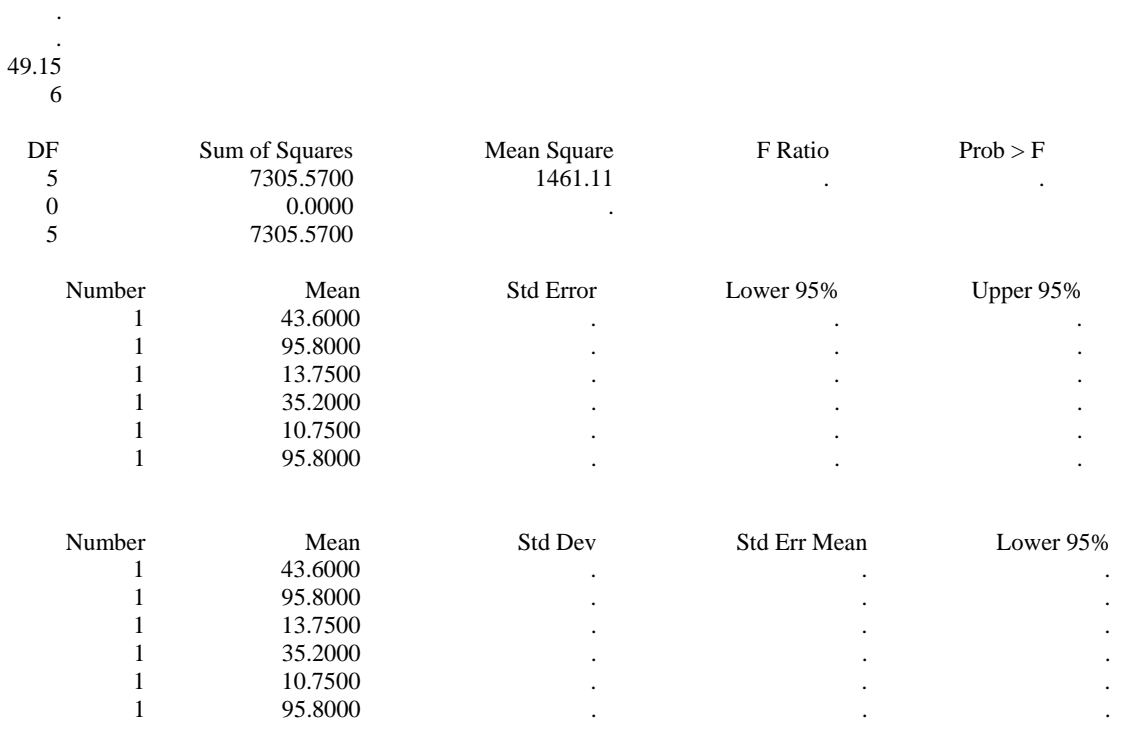

Upper 95\% 
WSRC-TR-2005-00166

SRNL-RPP-2005-00029

Revision 0

Exhibit A2. Statistical Comparisons of Preparation/Crucible Measurements by Element by Type of Sample

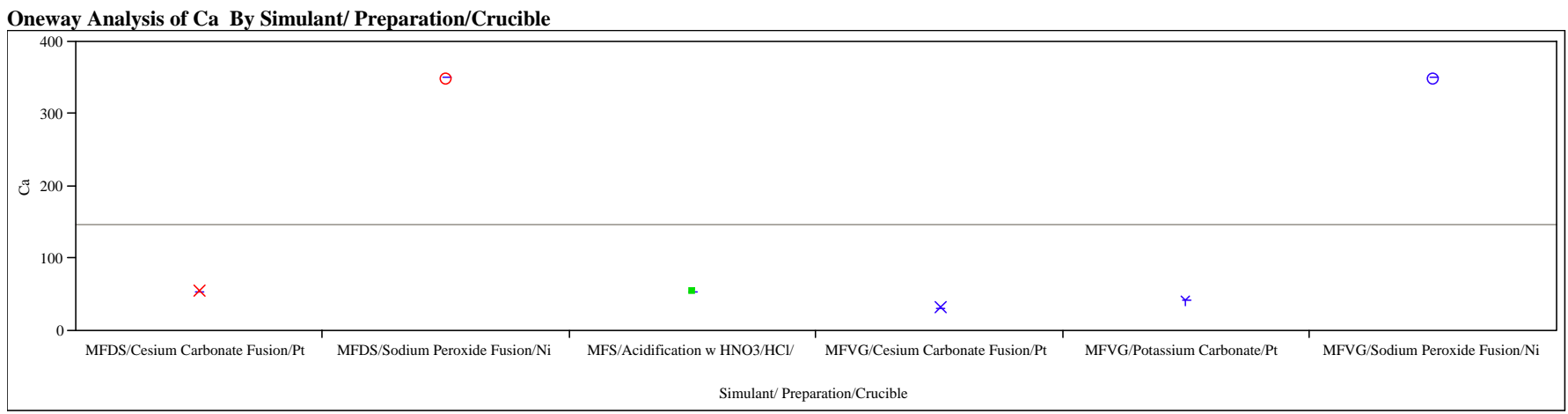

Oneway Anova

Summary of Fit

Rsquare

Adj Rsquare

Root Mean Square Erro

Mean of Response

Observations (or Sum Wgts)

Analysis of

Source

Simulant/ Preparation/Crucible

Error

C. Total

Means

MFDS/Cesium Carbonate Fusion/Pt

$\mathrm{FS} /$ Acidification w $\mathrm{HNO} / \mathrm{HCl} /$

MFVG/Cesium Carbonate Fusion/Pt

MFVG/Potassium Carbonate/Pt

MFVG/Sodium Peroxide Fusion/Ni

Std Error uses a pooled estimate of error variance

Means and Std Deviations

Level

MFDS/Cesium Carbonate Fusion/Pt

MFDS/Sodium Peroxide Fusion/Ni

MFS/Acidification w HNO3/HCl

MFVG/Cesium Carbonate Fusion/Pt

MFVG/Potassium Carbonate/Pt

MFVG/Sodium Peroxide Fusion/Ni

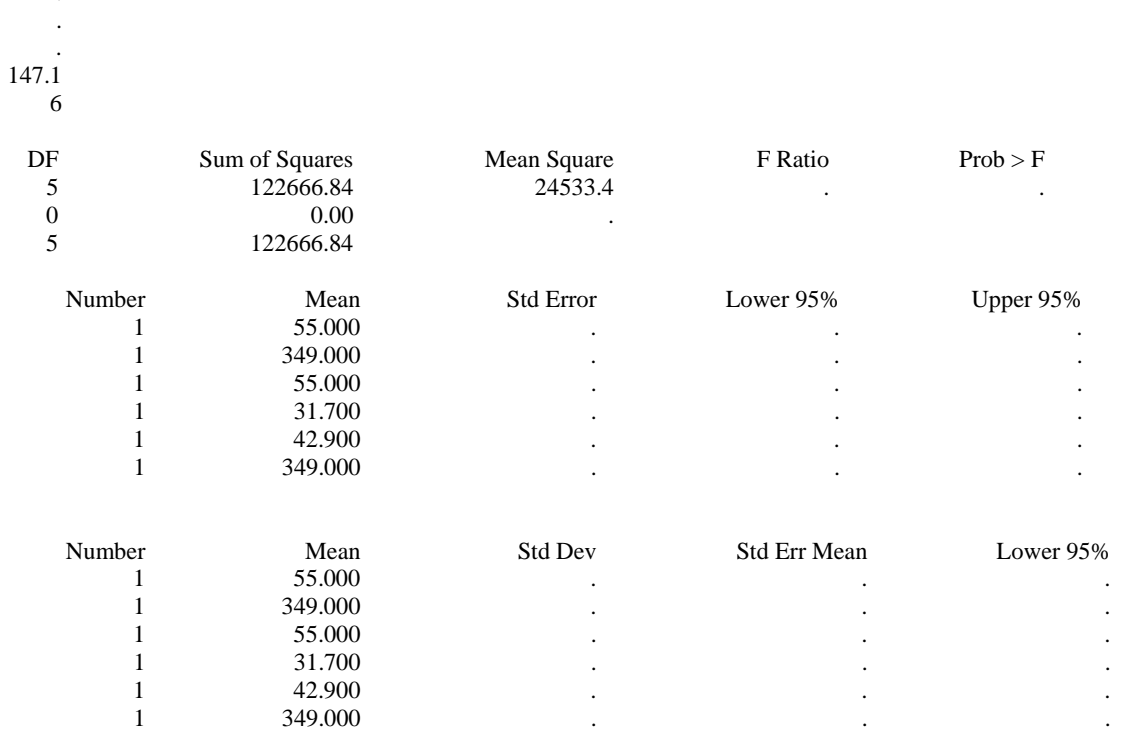

Upper 95\% 
WSRC-TR-2005-00166

SRNL-RPP-2005-00029

Revision 0

Exhibit A2. Statistical Comparisons of Preparation/Crucible Measurements by Element by Type of Sample

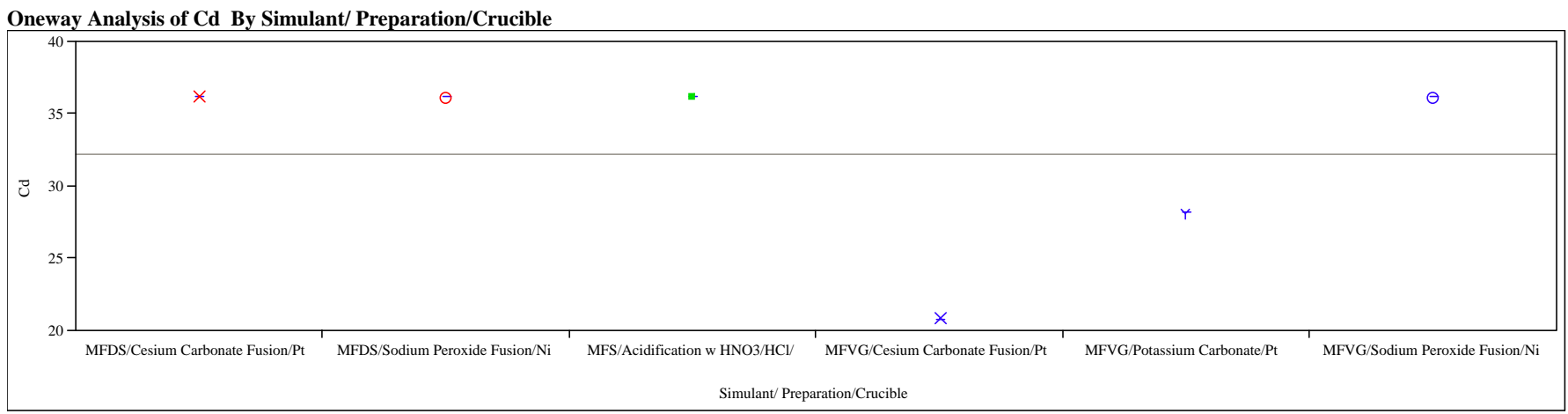

Oneway Anova

Summary of Fit

Rsquare

Adj Rsquare

Root Mean Square Erro

Mean of Response

Observations (or Sum Wgts)

Analysis of

Simulant/ Preparation/Crucible

Error

C. Total

Means for Oneway Anova

Level

Preparation/Crucible

MFDS/Cesium Carbonate Fusion/Pt

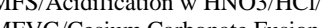

MFVG/Potassium Carbona/Pt

MFVG/Sodium Paboride

of error variance

viations

MFDel

MFDS/Cesium Carbonate Fusion/Pt

MFDS/Sodium Peroxide Fusion/Ni

MFS/Acidification w HNO3/HCl

MFVG/Cesium Carbonate Fusion/Pt

MFVG/Potassium Carbonate/Pt

MFVG/Sodium Peroxide Fusion/Ni

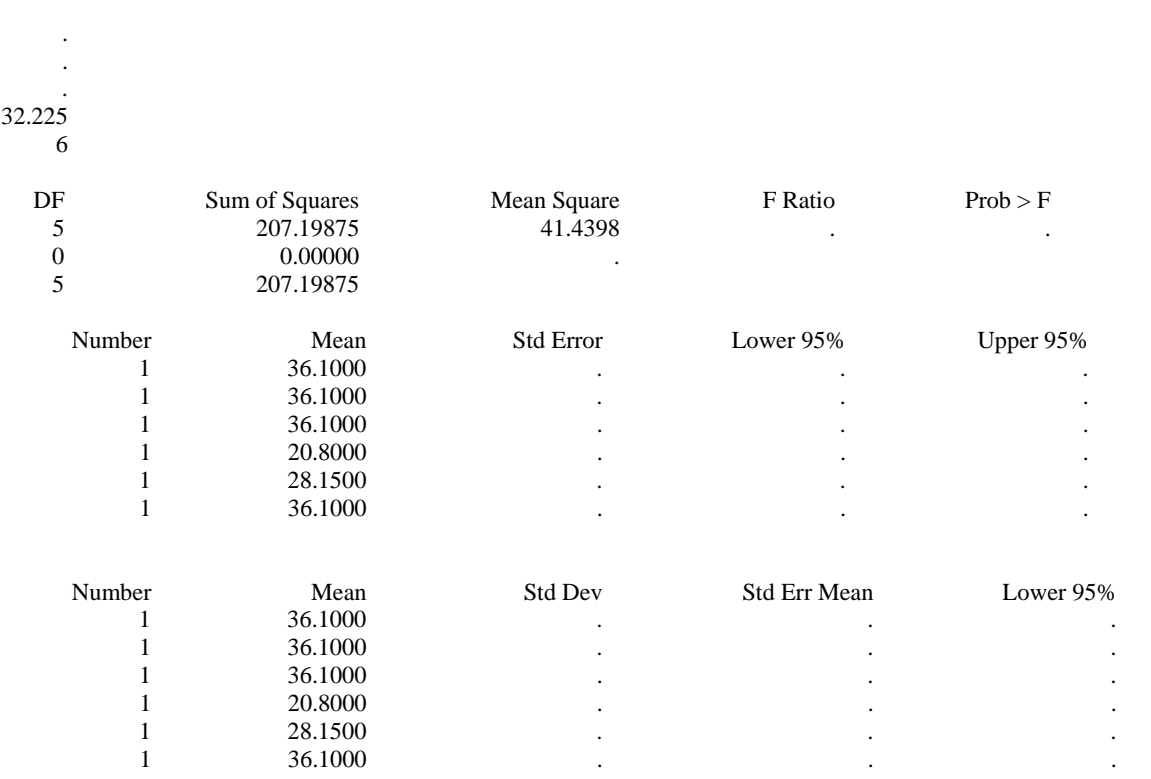

Upper 95\% 
WSRC-TR-2005-00166

SRNL-RPP-2005-00029

Revision 0

Exhibit A2. Statistical Comparisons of Preparation/Crucible Measurements by Element by Type of Sample

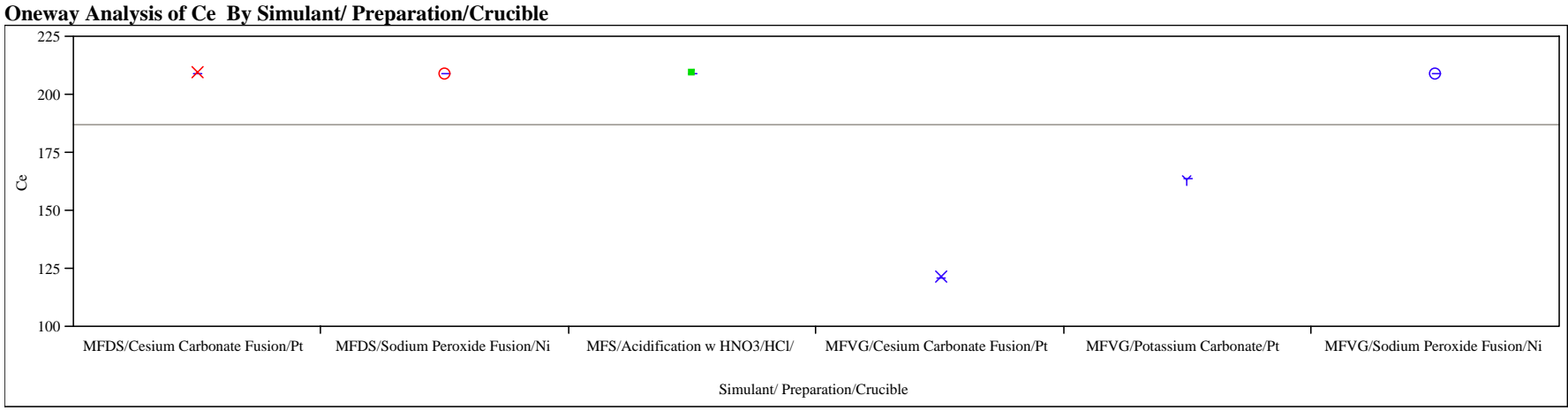

Oneway Anova

Summary of Fit

Rsquare

Adj Rsquare

oot Mean Square Error

Mean of Response

Observations (or Sum Wgts)

Analysis of

Simulant/ Preparation/Crucible

Error

C. Total

Means

MFDS/Cesium Carbonate Fusion/Pt

S

MFVG/Cesium Carbonate Fusion/Pt

MFVG/Potassium Carbonate/Pt

MFVG/Sodium Peroxide Fusion/Ni

Std Error uses a pooled estimate of error variance

Means and Std Deviations

Level

MFDS/Cesium Carbonate Fusion/Pt

MFDS/Sodium Peroxide Fusion/N

MFS/Acidification w HNO3/HCl

MFVG/Cesium Carbonate Fusion/Pt

MFVG/Potassium Carbonate/Pt

MFVG/Sodium Peroxide Fusion/Ni

\begin{tabular}{|c|c|c|c|c|}
\hline DF & Sum of Squares & Mean Square & F Ratio & Prob $>$ F \\
\hline 5 & 6933.208 & 1386.64 & & \\
\hline 0 & $-9.095 e-13$ & & & \\
\hline 5 & 6933.208 & & & \\
\hline Number & Mean & Std Error & Lower 95\% & Upper 95\% \\
\hline 1 & 209.500 & & . & . \\
\hline 1 & 209.500 & & . & . \\
\hline 1 & 209.500 & & . & . \\
\hline 1 & 121.000 & & . & . \\
\hline 1 & 163.500 & & . & . \\
\hline 1 & 209.500 & . & . & . \\
\hline Number & Mean & Std Dev & Std Err Mean & Lower 95\% \\
\hline 1 & 209.500 & & 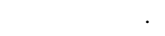 & \\
\hline 1 & 209.500 & . & . & \\
\hline 1 & 209.500 & & . & \\
\hline 1 & 121.000 & & . & \\
\hline 1 & 163.500 & & . & \\
\hline 1 & 209.500 & & . & \\
\hline
\end{tabular}

Upper 95\% 
WSRC-TR-2005-00166

SRNL-RPP-2005-00029

Revision 0

Exhibit A2. Statistical Comparisons of Preparation/Crucible Measurements by Element by Type of Sample

Oneway Analysis of $\mathrm{Cr}$ By Simulant/Preparation/Crucible

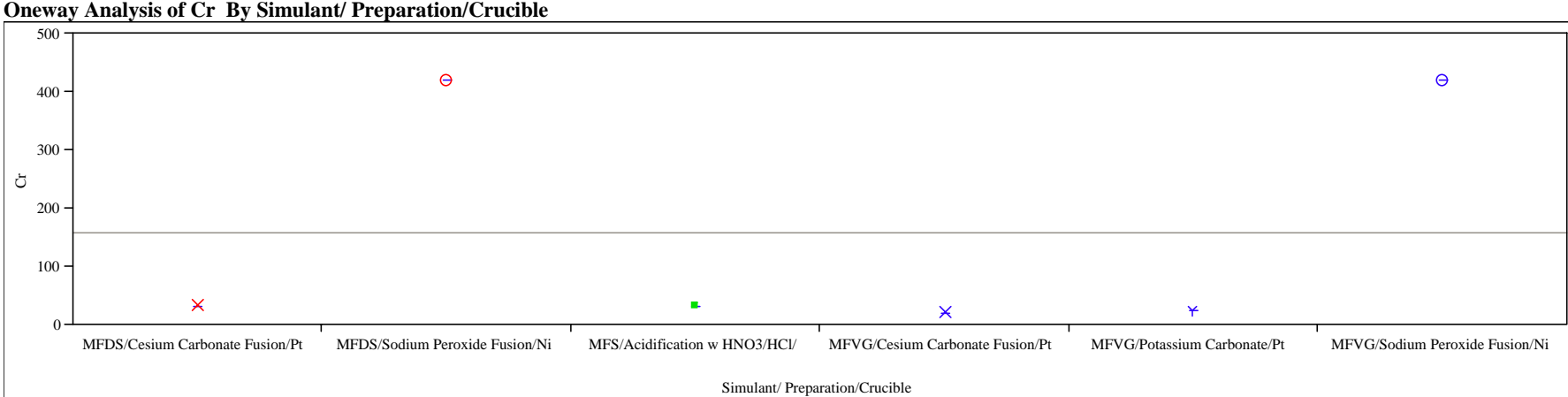

Oneway Anova

Summary of Fit

Rsquare

Adj Rsquare

Root Mean Square Error

Mean of Response

Observations (or Sum Wgts)

Analysis of Variance

Source

Simulant/ Preparation/Crucible

Error

Means for Oneway Anova

Level

MFDS/Cesium Carbonate Fusion/Pt

TFS/Aodidin Peroxide Fusion/Ni

MFVG/Cesiut W W HNO3/HCl/

MFVG/Cesium Carbonate Fusion/Pt

MFVG/Sodium Peabonater

MFV

Preparation/Crucible

Means and Std Deviations

Level

MFDS/Cesium Carbonate Fusion/Pt

TFS/Aodun Peroxide Fusion/N/

MFVG/Cin

MFVG Cesium Carbonate Fusion

MFVG/Solim Peroxide Fusior

\begin{tabular}{|c|c|c|c|c|}
\hline DF & Sum of Squares & Mean Square & F Ratio & Prob $>F$ \\
\hline 5 & 205270.41 & 41054.1 & . & . \\
\hline 0 & 2.9104e-11 & & & \\
\hline 5 & 205270.41 & & & \\
\hline Number & Mean & Std Error & Lower 95\% & Upper 95\% \\
\hline 1 & 33.100 & . & 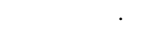 & 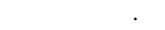 \\
\hline 1 & 420.000 & . & . & . \\
\hline 1 & 33.100 & . & . & . \\
\hline 1 & 19.050 & . & . & . \\
\hline 1 & 25.800 & . & . & . \\
\hline 1 & 420.000 & . & . & . \\
\hline Number & Mean & Std Dev & Std Err Mean & Lower 95\% \\
\hline 1 & 33.100 & . & 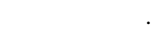 & \\
\hline 1 & 420.000 & . & . & . \\
\hline 1 & 33.100 & . & . & \\
\hline 1 & 19.050 & . & . & \\
\hline 1 & 25.800 & . & . & \\
\hline 1 & 420.000 & . & . & . \\
\hline
\end{tabular}

Upper 95\% 
WSRC-TR-2005-00166

SRNL-RPP-2005-00029

Revision 0

Exhibit A2. Statistical Comparisons of Preparation/Crucible Measurements by Element by Type of Sample

Oneway Analysis of Cu By Simulant/Preparation/Crucible

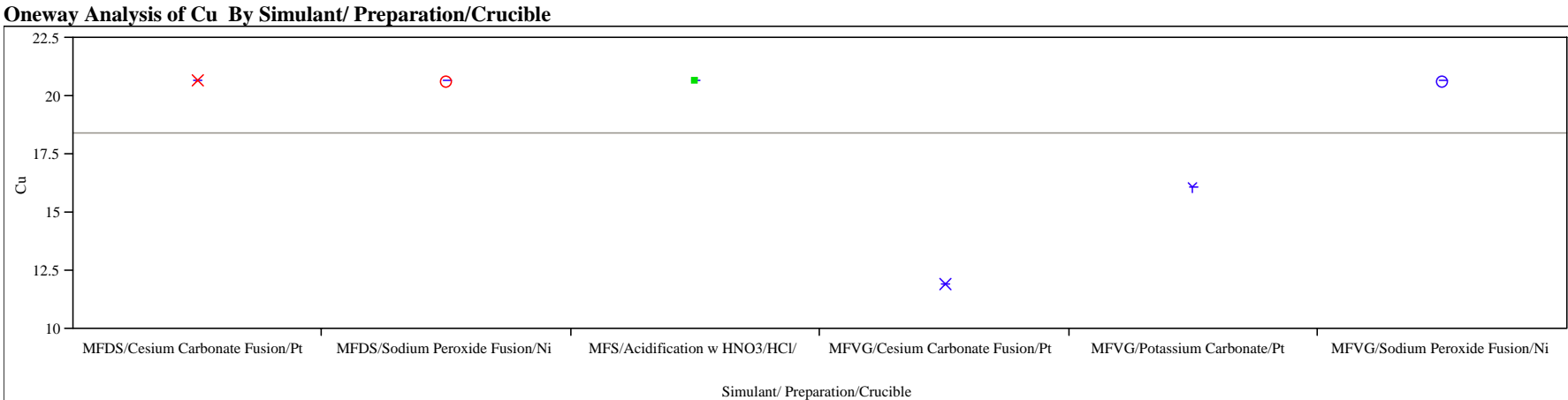

Oneway Anova

Simulant/Preparation/Crucible

ummary of Fit

Rsquare

Adj Rsquare

Root Mean Square Error

Mean of Response

Observations (or Sum Wgts)

Analysis of Variance

Source

Simulant/ Preparation/Crucible

Error

C. Total

Means for Oneway Anova

Level

MFDS/Cesium Carbonate Fusion/Pt

MFS/Acidifin Peroxide Fusion/Ni

MFVG/Cesiul Ca HNOB/HCl/

MFVG/Cesium Carbonate Fusion/Pt

MFVG/Sodium Peabonater

SFV

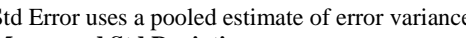

Means and Std Deviations

Level

MFDS/Cesium Carbonate Fusion/Pt

THS/Sodun Peroxide Fusion/Ni

MFVG/Cinit W wNOB/HCl/

MFVG/Cesium Carbonate Fusion/Pt

MFVG/Sodium Peroxide Fusion

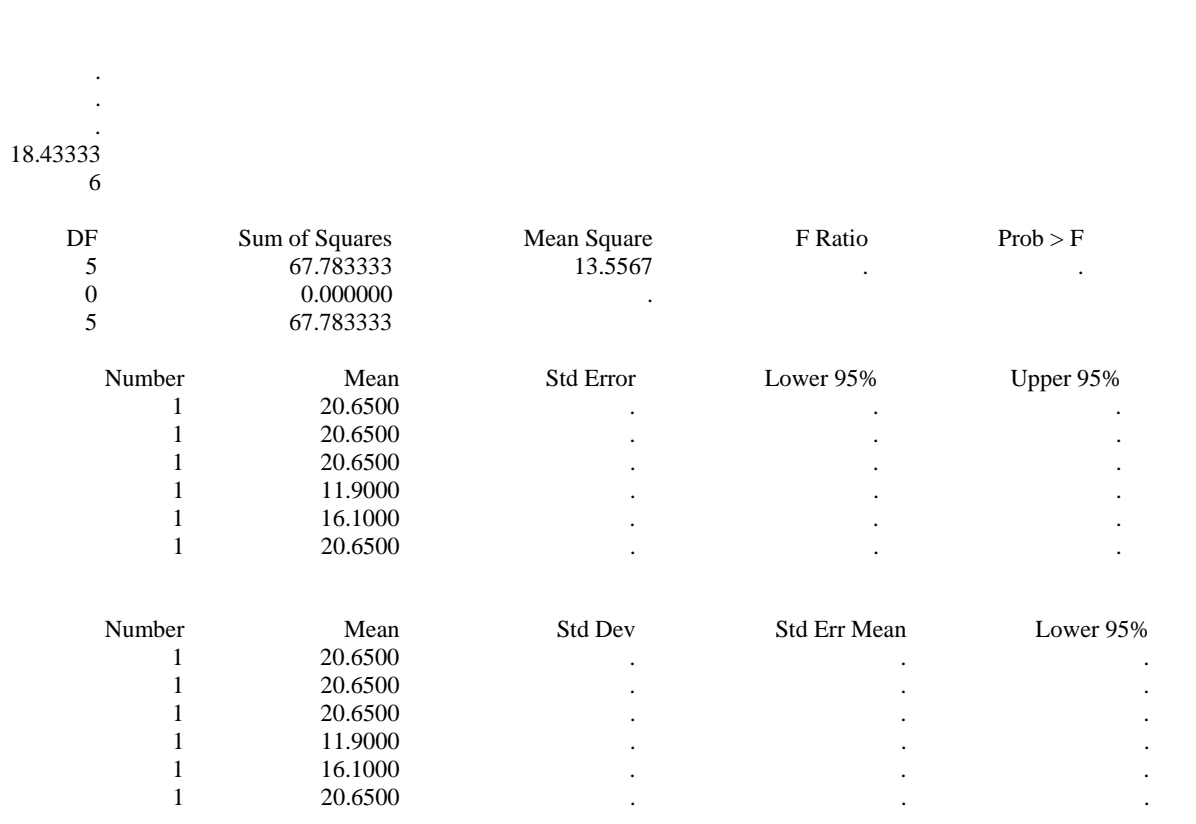


WSRC-TR-2005-00166

SRNL-RPP-2005-00029

Revision 0

Exhibit A2. Statistical Comparisons of Preparation/Crucible Measurements by Element by Type of Sample

Oneway Analysis of Fe By Simulant/Preparation/Crucible

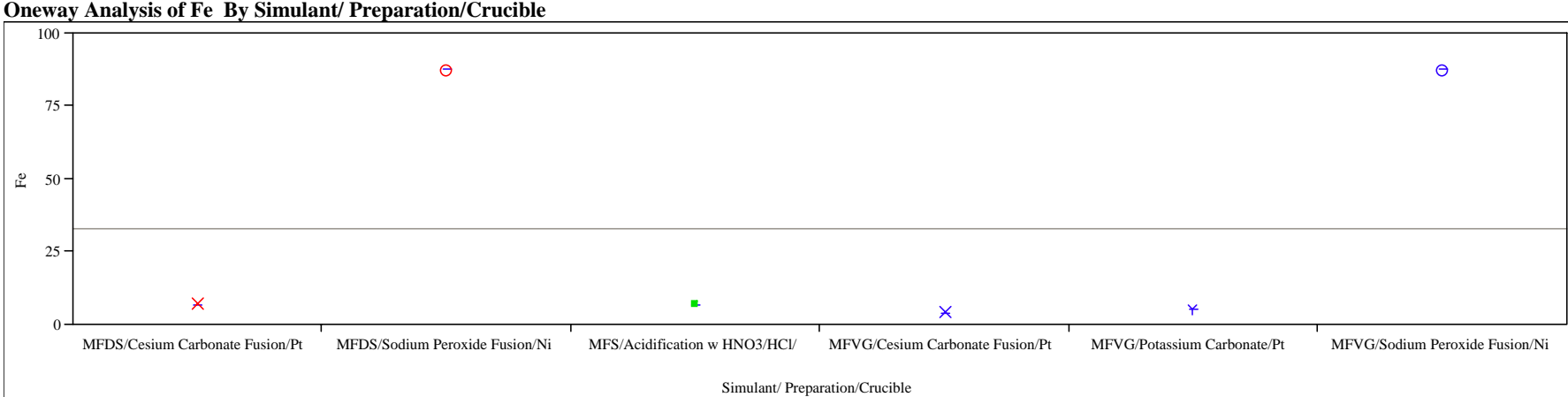

Oneway Anova

Summary of Fit

Rsquare

Adj Rsquare

Root Mean Square Error

Mean of Response

Observations (or Sum Wgts)

Analysis of Variance

Source

Simulant/ Preparation/Crucible

Error

Means for Oneway Anova

Level

MFDS/Cesium Carbonate Fusion/Pt

TFS/Aodum Peroxide Fusion//

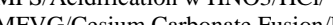

MFVG/Cesium Carbonate Fusion/

MFVG/Sodium Pexbonatert

MFV/Sodium Peroxide Fusion/Ni

t/ Preparation/Crucible

Means and Std Deviations

Level

MFDS/Cesium Carbonate Fusion/Pt

MFS/Sodum Peroxide Fusion/Ni

MFVG/Cesim W HNO3/HCl/

MFVG/Cesiun Catbonate Fusion

MFVG/Solim Peroxide Fusion

\begin{tabular}{|c|c|c|c|c|}
\hline DF & Sum of Squares & Mean Square & F Ratio & Prob $>$ F \\
\hline 5 & 8870.7121 & 1774.14 & . & . \\
\hline 0 & 0.0000 & & & \\
\hline 5 & 8870.7121 & & & \\
\hline Number & Mean & Std Error & Lower 95\% & Upper 95\% \\
\hline 1 & 7.1000 & . & . & Prentos. \\
\hline 1 & 87.5000 & . & . & . \\
\hline 1 & 7.1000 & . & . & . \\
\hline 1 & 4.1000 & . & . & . \\
\hline 1 & 5.5500 & . & . & . \\
\hline 1 & 87.5000 & . & . & . \\
\hline Number & Mean & Std Dev & Std Err Mean & Lower 95\% \\
\hline 1 & 7.1000 & & & \\
\hline 1 & 87.5000 & . & . & . \\
\hline 1 & 7.1000 & . & . & . \\
\hline 1 & 4.1000 & . & . & \\
\hline 1 & 5.5500 & . & . & . \\
\hline 1 & 87.5000 & . & . & \\
\hline
\end{tabular}

Upper 95\% 
WSRC-TR-2005-00166

SRNL-RPP-2005-00029

Revision 0

Exhibit A2. Statistical Comparisons of Preparation/Crucible Measurements by Element by Type of Sample

Oneway Analysis of K By Simulant/ Preparation/Crucible

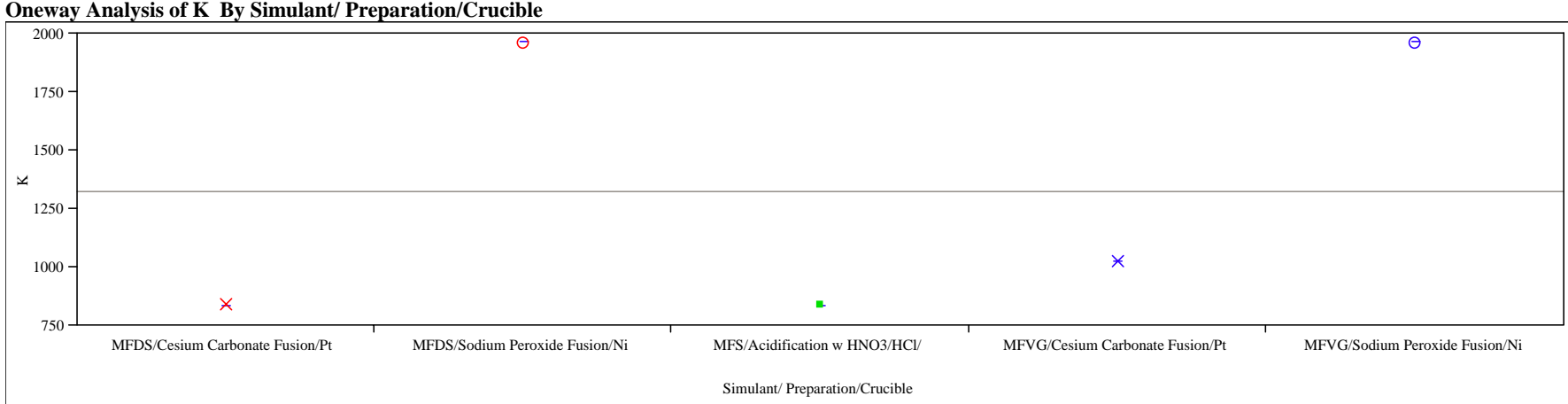

Missing Rows

eway Anova

Summary of Fit

Rsquare

Adj Rsquare

Root Mean Square Erro

Mean of Response

Observations (or Sum Wgts)

Analysis of Variance

Source

Simulant/ Preparation/Crucible

Error

C. Total

Means for Oneway Anova

Level

MFDS/Cesium Carbonate Fusion/Pt

MFS/Sodium Peroxide Fusion/N/

MFVG/Cesium $\mathrm{W}$ HNO3/HCl/

MFVG/Sodium Peroxide Fusion/Pt

S G/Sodiun Peroxide Fusion/Ni

Ste Enor uses a pooled estimate of enror

Means and Std Deviations

Level

MFDS/Cesium Carbonate Fusion/Pt

FFS/Acidifin Peroxide Fusion/MV

MFVG/Cin w H H

MFVG/Sodium Peroxide Fusion/Ni

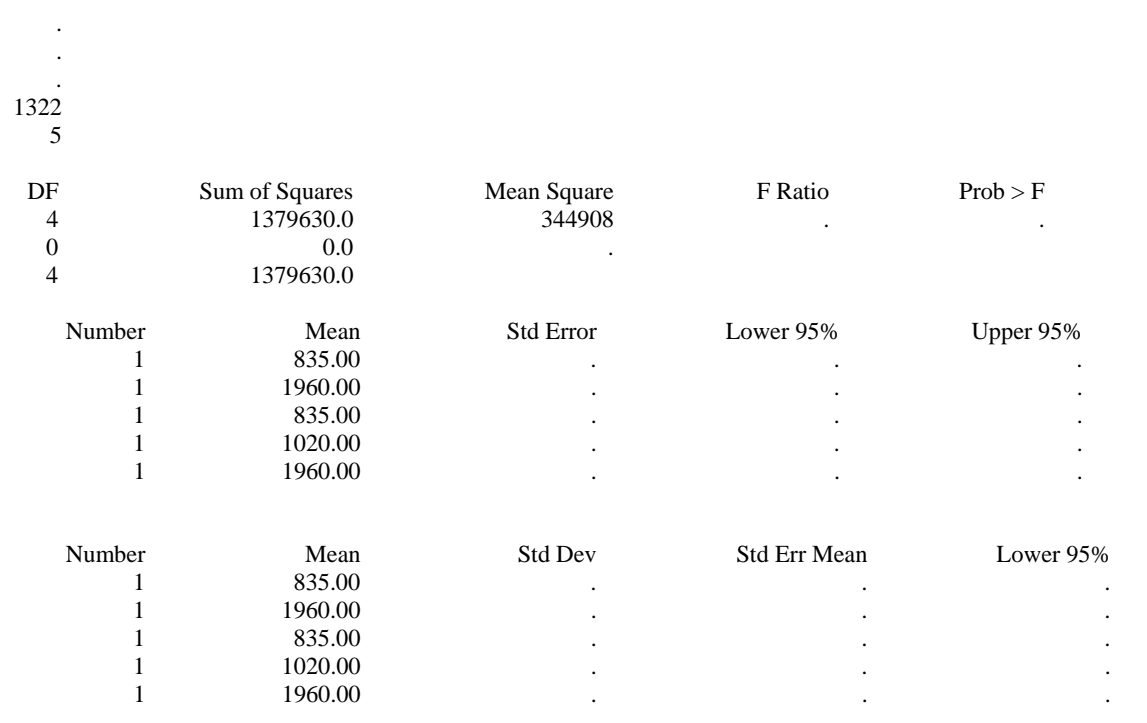

Upper 95\% 
WSRC-TR-2005-00166

SRNL-RPP-2005-00029

Revision 0

Exhibit A2. Statistical Comparisons of Preparation/Crucible Measurements by Element by Type of Sample

Oneway Analysis of La By Simulant/ Preparation/Crucible

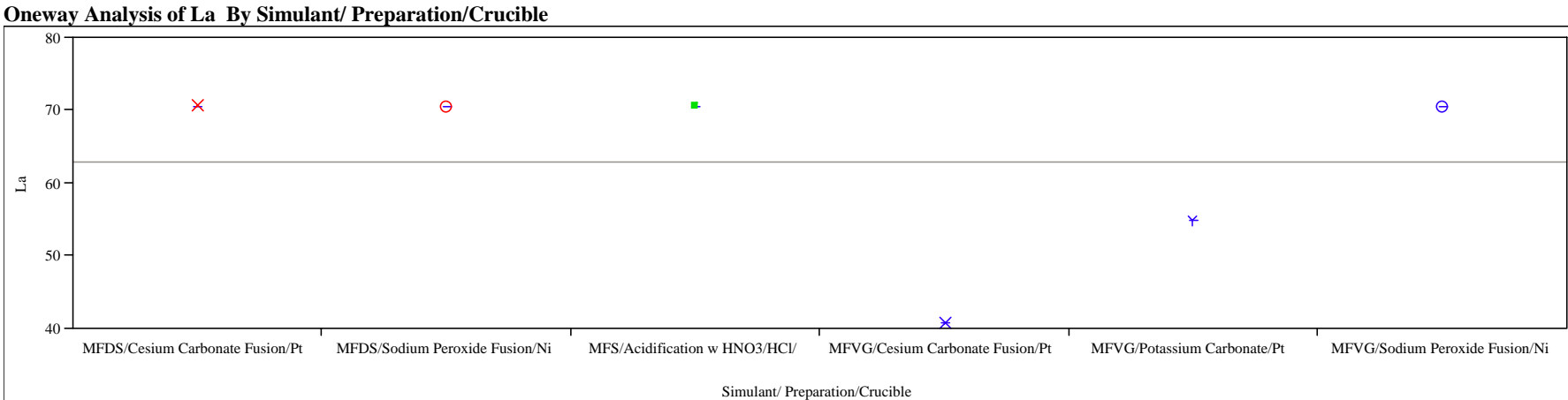

Oneway Anova

Simulant/Preparation/Crucible

ummary of Fit

Rsquare

Adj Rsquare

Root Mean Square Error

Mean of Response

Observations (or Sum Wgts)

Analysis of Variance

Source

Simulant/ Preparation/Crucible

Error

C. Total

Means for Oneway Anova

Level

MFDS/Cesium Carbonate Fusion/Pt

MFS/Aodin Peroxide Fusin/N/

MFVG/C i C W HNOB/HCl/

MFVG/Cesium Carbonate Fusion/Pt

MFVG/Sodium Pexbonatert

MFV/Sodium Peroide Fusion/Ni

Stror uniance

Means and Std Deviatio

MFDel

MFDS/Cesium Carbonate Fusion/Pt

TFS/Aodum Peroxide Fusion/N/

MFVG/Cinit W WNOB/HCI/

MFVG/Cosium Carbonate Fusion/Pt

MFVG/Sodium Peroxide Fusion

\begin{tabular}{|c|c|c|c|c|}
\hline \multicolumn{5}{|l|}{$\begin{array}{r}62.93333 \\
6\end{array}$} \\
\hline DF & Sum of Squares & Mean Square & F Ratio & Prob $>$ F \\
\hline 5 & 790.73333 & 158.147 & & 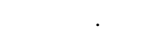 \\
\hline 0 & 0.00000 & & & \\
\hline 5 & 790.73333 & & & \\
\hline Number & Mean & Std Error & Lower 95\% & Upper 95\% \\
\hline 1 & 70.5000 & . & 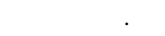 & . \\
\hline 1 & 70.5000 & . & . & . \\
\hline 1 & 70.5000 & . & . & . \\
\hline 1 & 40.6000 & . & . & . \\
\hline 1 & 55.0000 & . & . & . \\
\hline 1 & 70.5000 & . & . & . \\
\hline Number & Mean & Std Dev & Std Err Mean & Lower 95\% \\
\hline 1 & 70.5000 & & . & \\
\hline 1 & 70.5000 & . & . & \\
\hline 1 & 70.5000 & . & . & \\
\hline 1 & 40.6000 & . & . & \\
\hline 1 & 55.0000 & . & . & . \\
\hline 1 & 70.5000 & . & . & \\
\hline
\end{tabular}


WSRC-TR-2005-00166

SRNL-RPP-2005-00029

Revision 0

Exhibit A2. Statistical Comparisons of Preparation/Crucible Measurements by Element by Type of Sample

Oneway Analysis of Li By Simulant/Preparation/Crucible

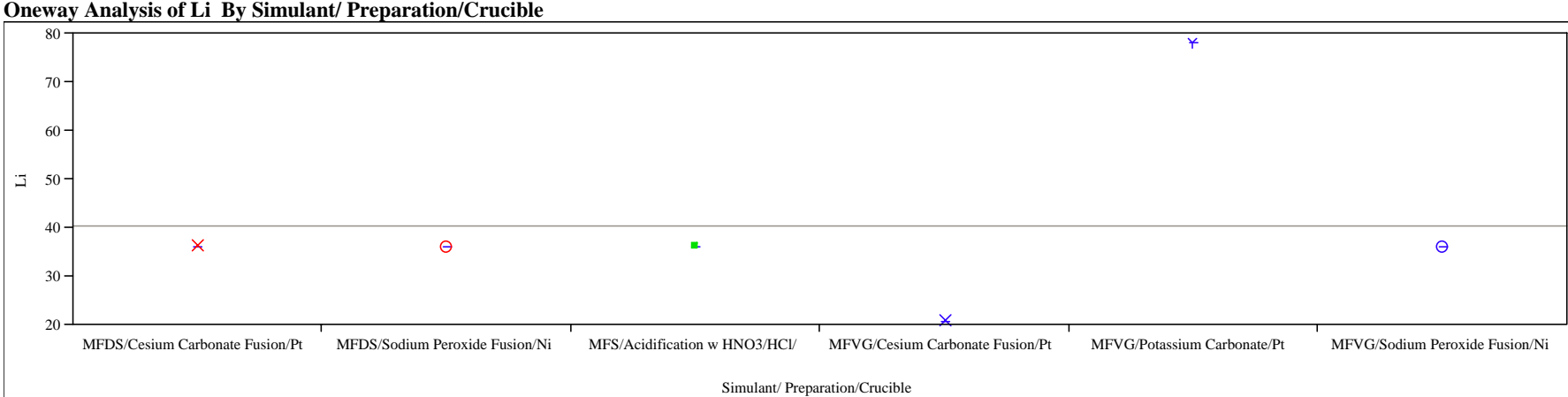

Oneway Anova

Summary of Fit

Rsquare

Adj Rsquare

Root Mean Square Error

Mean of Response

Observations (or Sum Wgts)

Analysis of Variance

Simulant/ Preparation/Crucible

Error

Means for Oneway Anova

Level

MFDS/Cesium Carbonate Fusion/Pt

MFS/Aodidid Peroxide Fusion/N/

MFVG/C i C W HNO3/HCl/

MFVG/Cesium Carbonate Fusion/Pt

MFVG/Sodium Paboster

MFV/Sodium Peroxide Fusion/Ni

Preparation/Crucible

Means and Std Deviations

Level

MFDS/Cesium Carbonate Fusion/Pt

TFS/Aodum Peroxide Fusion/N/

MFVG/Cinit W WNOB/HCl/

MFVG/Cosium Carbonate Fusion

MFVG/Sodium Peroxide Fusion

\begin{tabular}{|c|c|c|c|c|}
\hline DF & Sum of Squares & Mean Square & F Ratio & Prob $>$ F \\
\hline 5 & 1871.7733 & 374.355 & . & . \\
\hline 0 & $2.2737 \mathrm{e}-13$ & & & \\
\hline 5 & 1871.7733 & & & \\
\hline Number & Mean & Std Error & Lower 95\% & Upper 95\% \\
\hline 1 & 36.1000 & . & . & Prentos. \\
\hline 1 & 36.1000 & . & . & . \\
\hline 1 & 36.1000 & . & . & . \\
\hline 1 & 20.8000 & . & . & . \\
\hline 1 & 78.0000 & . & . & . \\
\hline 1 & 36.1000 & . & . & . \\
\hline Number & Mean & Std Dev & Std Err Mean & Lower 95\% \\
\hline 1 & 36.1000 & & & \\
\hline 1 & 36.1000 & . & . & \\
\hline 1 & 36.1000 & . & . & . \\
\hline 1 & 20.8000 & . & . & \\
\hline 1 & 78.0000 & . & . & . \\
\hline 1 & 36.1000 & . & . & \\
\hline
\end{tabular}

Upper 95\% 
WSRC-TR-2005-00166

SRNL-RPP-2005-00029

Revision 0

Exhibit A2. Statistical Comparisons of Preparation/Crucible Measurements by Element by Type of Sample

Oneway Analysis of Mg By Simulant/Preparation/Crucible

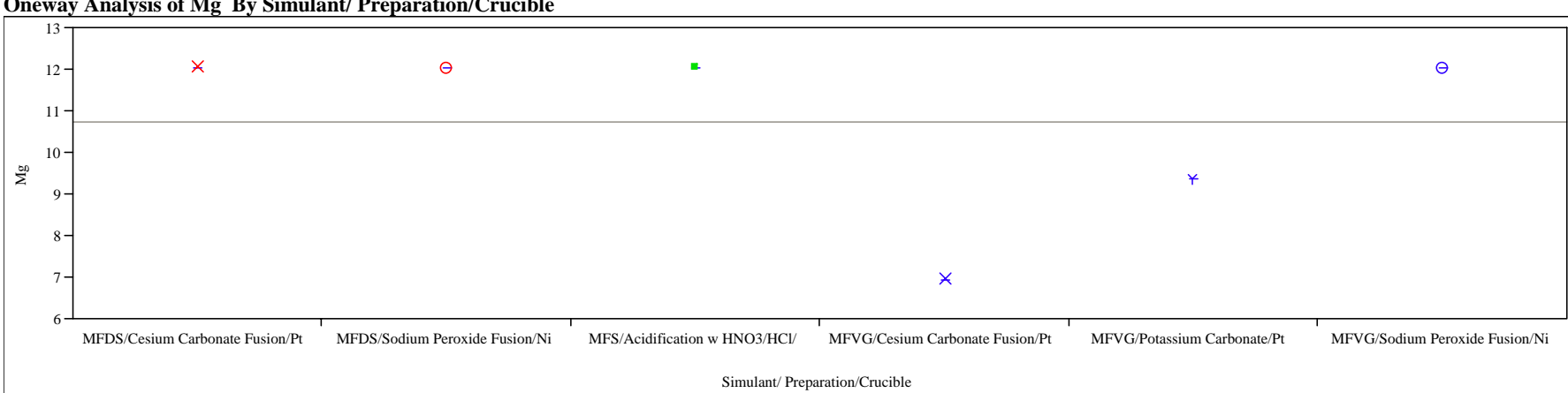

Oneway Anova

Summary of Fit

Rsquare

Adj Rsquare

Root Mean Square Erro

Mean of Response

Observations (or Sum Wgts)

Analysis of Variance

Source

Simulant/ Preparation/Crucible

Error

Means for Oneway Anova

Level

MFDS/Cesium Carbonate Fusion/Pt

TFS/Aodidi Peroxide Fusion/Ni

MFVG/Cesim CabNo3/HCl

MFVG/Cesium Carbonate Fusion/Pt

MFVG/Sodium Peronde Fusio

SFV

Preparation/Cruc

Means and Std Deviations

Level

MFDS/Cesium Carbonate Fusion/Pt

TFS/Aodun Peroxide Fusion/N/

MFVG/Cilo w

MFVG Cesium Carbonate Fusion

MFVG/Sodium Peroxide Fusion

\begin{tabular}{|c|c|c|c|c|}
\hline \multicolumn{5}{|l|}{$\begin{array}{r}10.75833 \\
6\end{array}$} \\
\hline $\mathrm{DF}$ & Sum of Squares & Mean Square & F Ratio & Prob $>$ F \\
\hline 5 & 23.022083 & 4.60442 & . & . \\
\hline 0 & 3.5527e-15 & & & \\
\hline 5 & 23.022083 & & & \\
\hline Number & Mean & Std Error & Lower 95\% & Upper 95\% \\
\hline 1 & 12.0500 & . & 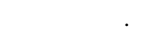 & . \\
\hline 1 & 12.0500 & . & . & . \\
\hline 1 & 12.0500 & . & . & . \\
\hline 1 & 6.9500 & . & . & . \\
\hline 1 & 9.4000 & . & . & . \\
\hline 1 & 12.0500 & . & . & . \\
\hline Number & Mean & Std Dev & Std Err Mean & Lower 95\% \\
\hline 1 & 12.0500 & . & . & \\
\hline 1 & 12.0500 & . & . & \\
\hline 1 & 12.0500 & . & . & \\
\hline 1 & 6.9500 & . & . & \\
\hline 1 & 9.4000 & . & . & \\
\hline 1 & 12.0500 & . & . & . \\
\hline
\end{tabular}

Upper 95\% 
WSRC-TR-2005-00166

SRNL-RPP-2005-00029

Revision 0

Exhibit A2. Statistical Comparisons of Preparation/Crucible Measurements by Element by Type of Sample

Oneway Analysis of Mn By Simulant/Preparation/Crucible

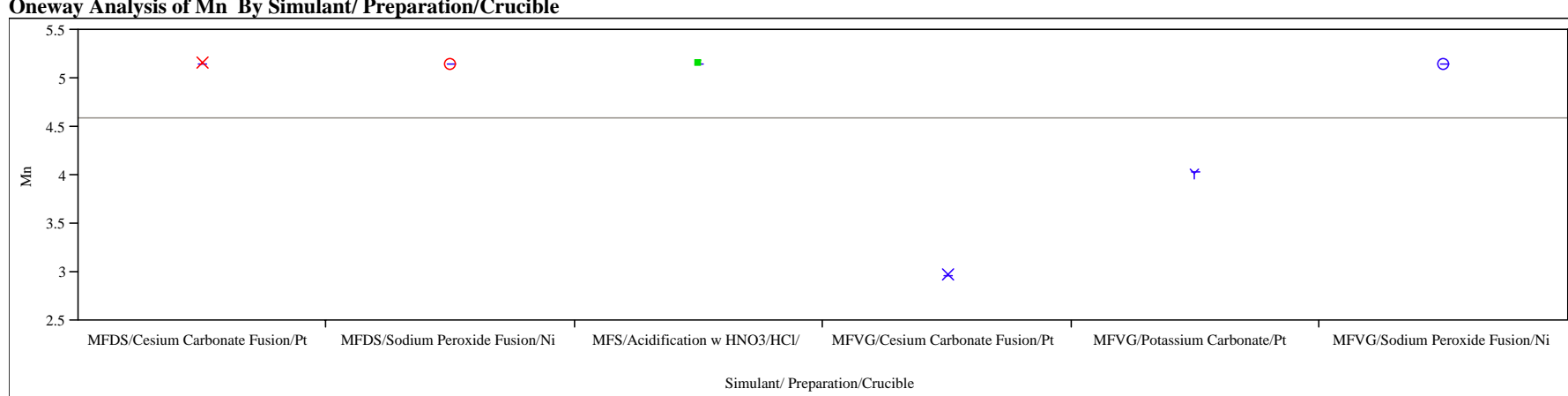

Oneway Anova

Simulant/Preparation/Crucible

ummary of Fit

Rsquare

Adj Rsquare

Root Mean Square Error

Mean of Response

Observations (or Sum Wgts)

Analysis of Variance

Source

Simulant/ Preparation/Crucible

Error

Means for Oneway Anova

Level

MFDS/Cesium Carbonate Fusion/Pt

MFS/Aodin Peroxide Fusion/N/

(1)

MFVG/Cesium Carbonate Fusion/Pt

MFVG/Sodium PerbonatPr

ST/Sodium Peroide Fusion/Ni

Std Erisor uses a poever

Means and Std Deviations

MFDel

MFDS/Cesium Carbonate Fusion/Pt

TFS/Aodum Peroxide Fusion/Ny

MFVG/Cesim W WNOB/HCl/

MFVG Cosium Carbonate Fusion

MFVG/Solim Peroxide Fusiont

\begin{tabular}{|c|c|c|c|c|}
\hline DF & Sum of Squares & Mean Square & F Ratio & Prob $>$ F \\
\hline 5 & 4.2032833 & 0.840657 & . & . \\
\hline 0 & 0.0000000 & & & \\
\hline 5 & 4.2032833 & & & \\
\hline Number & Mean & Std Error & Lower 95\% & Upper 95\% \\
\hline 1 & 5.15000 & . & . & . \\
\hline 1 & 5.15000 & . & . & . \\
\hline 1 & 5.15000 & . & . & . \\
\hline 1 & 2.97000 & . & . & . \\
\hline 1 & 4.02000 & . & . & . \\
\hline 1 & 5.15000 & . & . & . \\
\hline Number & Mean & Std Dev & Std Err Mean & Lower $95 \%$ \\
\hline 1 & 5.15000 & . & . & שv \\
\hline 1 & 5.15000 & . & . & . \\
\hline 1 & 5.15000 & . & . & \\
\hline 1 & 2.97000 & . & . & \\
\hline 1 & 4.02000 & . & . & \\
\hline 1 & 5.15000 & . & . & . \\
\hline
\end{tabular}

Upper 95\% 
WSRC-TR-2005-00166

SRNL-RPP-2005-00029

Revision 0

Exhibit A2. Statistical Comparisons of Preparation/Crucible Measurements by Element by Type of Sample

Oneway Analysis of Mo By Simulant/Preparation/Crucible

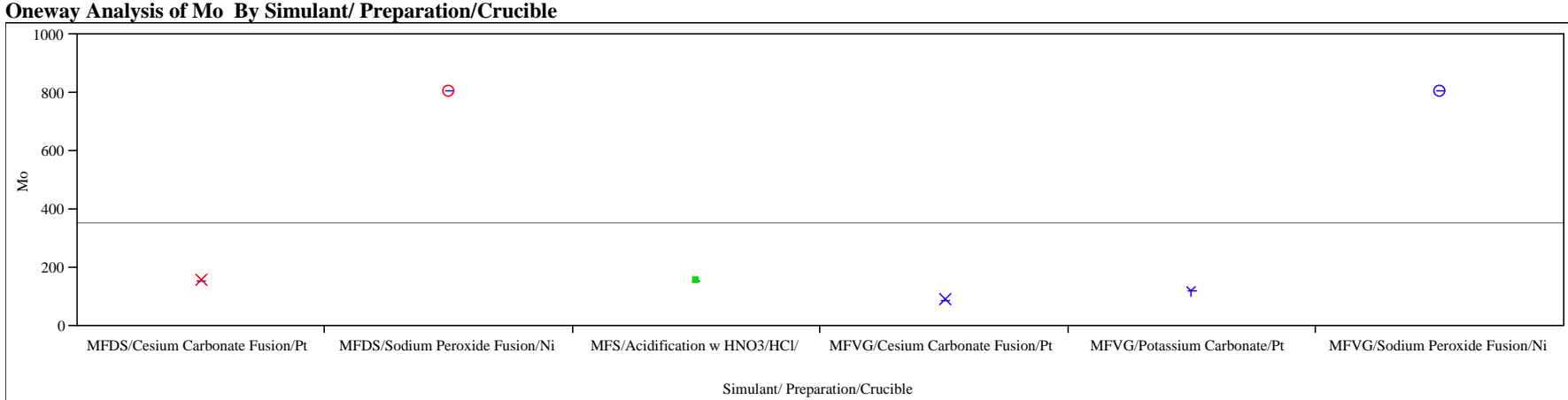

Oneway Anova

ummary of Fit

Rsquare

Adj Rsquare

Root Mean Square Error

Mean of Response

Observations (or Sum Wgts)

Analysis of Variance

Source

Simulant/ Preparation/Crucible

Error

Means for Oneway Anova

Level

MFDS/Cesium Carbonate Fusion/Pt

MFS/Aodidin Peroxide Fusion//

MFVG/C i Cation wh/

MFVG/Cesium Carbonate Fusion/Pt

MFVG/Sodium Pexhonte Fusio

Ster/Sodium Peroxide Fusion/Ni

Preparation/Cruc

Means and Std Deviations

Level

MFDS/Cesium Carbonate Fusion/Pt

TFS/Aodun Peroxide Fusion/N/

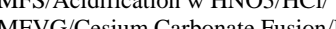

MFVG/Cosium Carbonate Fusion

MFVG/Sodium Peroxide Fusion

\begin{tabular}{|c|c|c|c|c|}
\hline \multicolumn{5}{|l|}{$\begin{array}{r}354.3333 \\
6\end{array}$} \\
\hline DF & Sum of Squares & Mean Square & F Ratio & Prob $>$ F \\
\hline 5 & 614889.8 & 122978 & . & . \\
\hline 0 & $-1.164 \mathrm{e}-10$ & & & \\
\hline 5 & 614889.8 & & & \\
\hline Number & Mean & Std Error & Lower 95\% & Upper 95\% \\
\hline 1 & 153.000 & & 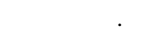 & . \\
\hline 1 & 806.000 & & . & . \\
\hline 1 & 153.000 & & . & . \\
\hline 1 & 88.500 & . & . & . \\
\hline 1 & 119.500 & & . & . \\
\hline 1 & 806.000 & . & . & . \\
\hline Number & Mean & Std Dev & Std Err Mean & Lower 95\% \\
\hline 1 & 153.000 & & . & \\
\hline 1 & 806.000 & & . & \\
\hline 1 & 153.000 & & . & \\
\hline 1 & 88.500 & & . & \\
\hline 1 & 119.500 & & . & \\
\hline 1 & 806.000 & & . & \\
\hline
\end{tabular}

Upper 95\% 
WSRC-TR-2005-00166

SRNL-RPP-2005-00029

Revision 0

Exhibit A2. Statistical Comparisons of Preparation/Crucible Measurements by Element by Type of Sample

Oneway Analysis of Na By Simulant/Preparation/Crucible

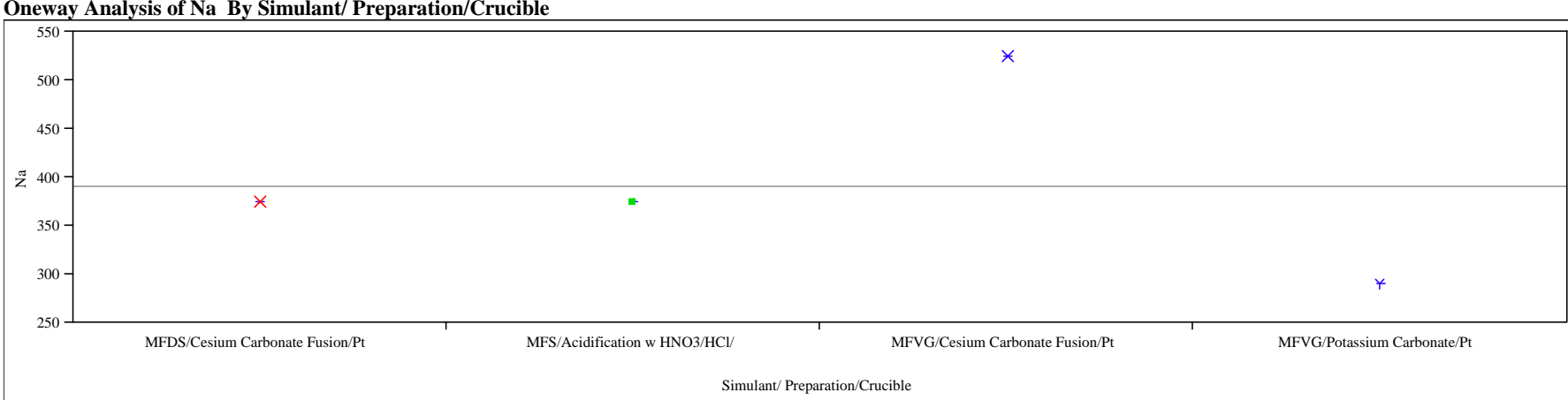

Missing Rows

nulant/Preparation/Crucis

Summary of Fit

Rsquare

Adj Rsquare

Root Mean Square Error

Mean of Response

Observations (or Sum Wgts)

Analysis of Variance

Source

Simulant/ Preparation/Crucible

C. Total

Means for Oneway Anova

Level

MFDS/Cesium Carbonate Fusion/Pt

MFS/Acidification w HNO3/HCl/

MFVG Cesium Carbonate Fusion/Pt

MFV G/Potassium Carbonate/Pt

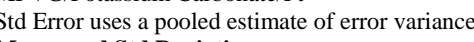

Means and Std Deviations

Level

MFDS/Cesium Carbonate Fusion/Pt

MFS/Acidification w HNO3/HCl/

MFV Cesium Carbonate Fusion/Pt

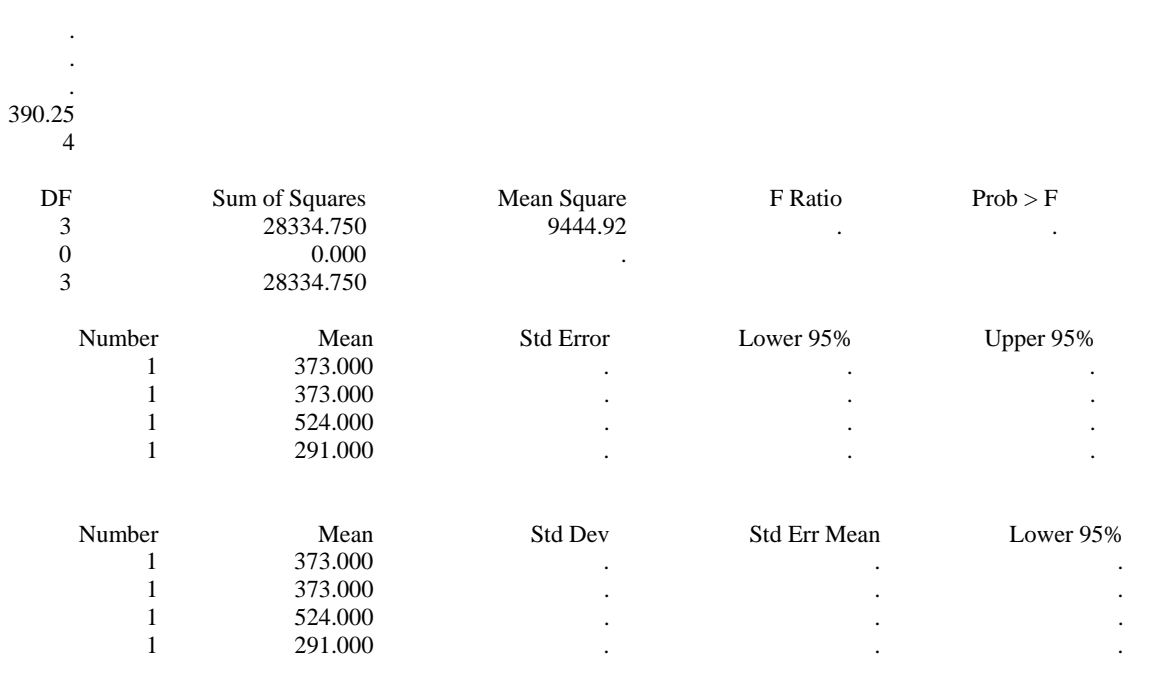

Upper 95\% 
WSRC-TR-2005-00166

SRNL-RPP-2005-00029

Revision 0

Exhibit A2. Statistical Comparisons of Preparation/Crucible Measurements by Element by Type of Sample

Oneway Analysis of Ni By Simulant/Preparation/Crucible

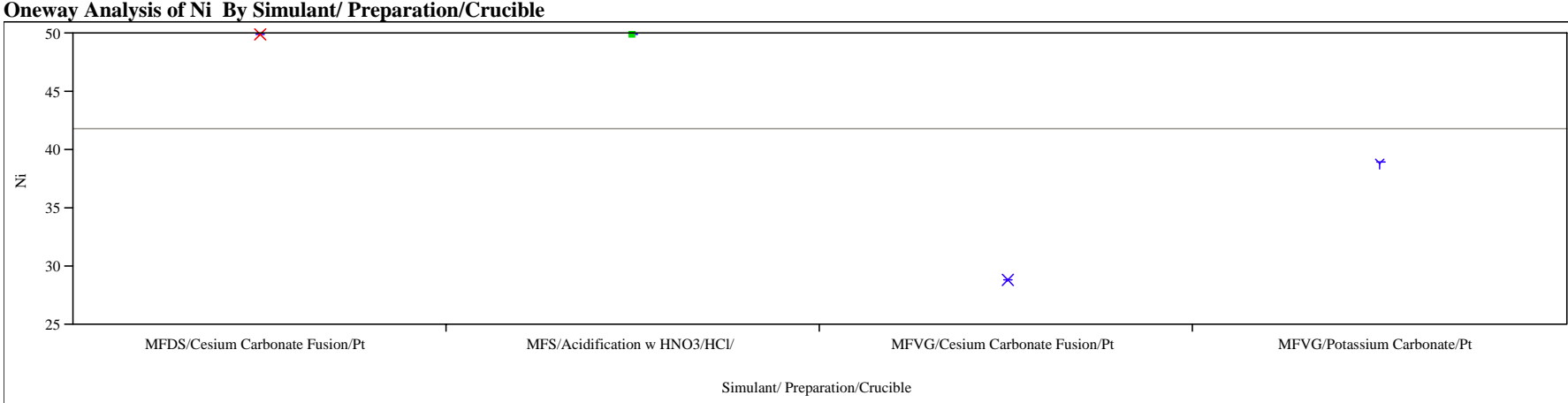

Missing Rows

ulant/Preparation/Cruciteste

Summary of Fit

Rsquare

Adj Rsquare

Root Mean Square Error

Mean of Response

Observations (or Sum Wgts)

Analysis of Variance

Source

Simulant/ Preparation/Crucible

C. Total

Means for Oneway Anova

Level

MFDS/Cesium Carbonate Fusion/Pt

MFS/Acidification w HNO3/HCl/

MFVG Cesium Carbonae Fusion/Pt

MFVG/Potassium Carbonate/Pt

Serror variance

Means and Std Deviation

Level

MFDS/Cesium Carbonate Fusion/Pt

MFS/Acidification w HNO3/HCl/

MFVG Postassiur Canate Fusion

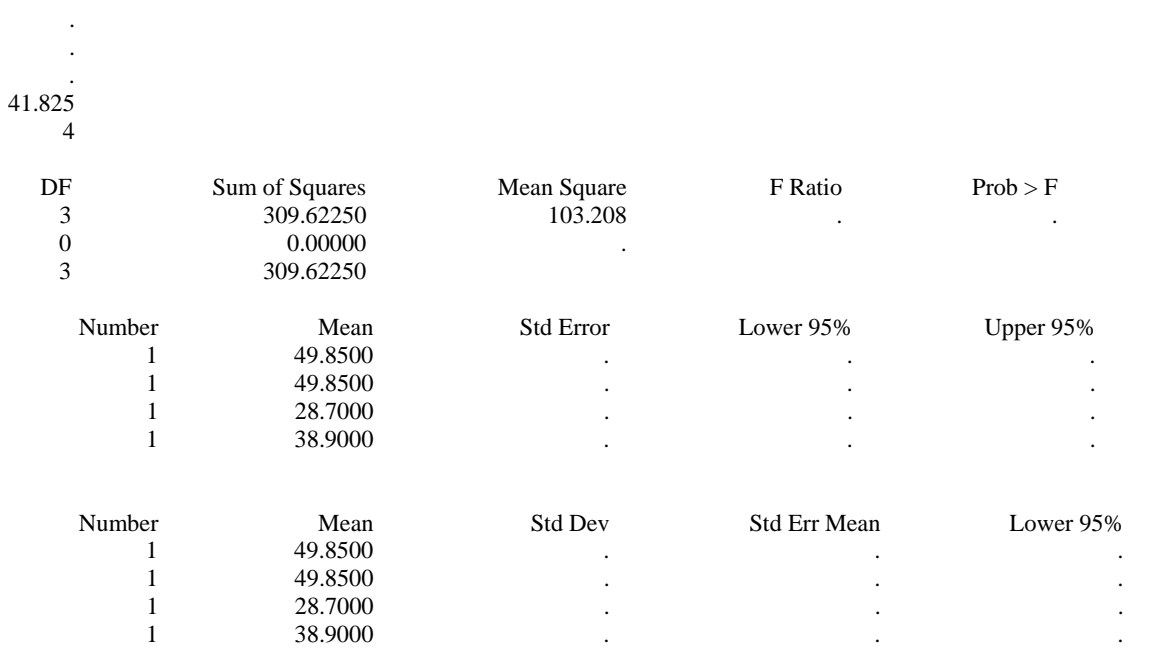

Upper 95\% 
WSRC-TR-2005-00166

SRNL-RPP-2005-00029

Revision 0

Exhibit A2. Statistical Comparisons of Preparation/Crucible Measurements by Element by Type of Sample

Oneway Analysis of P By Simulant/Preparation/Crucible

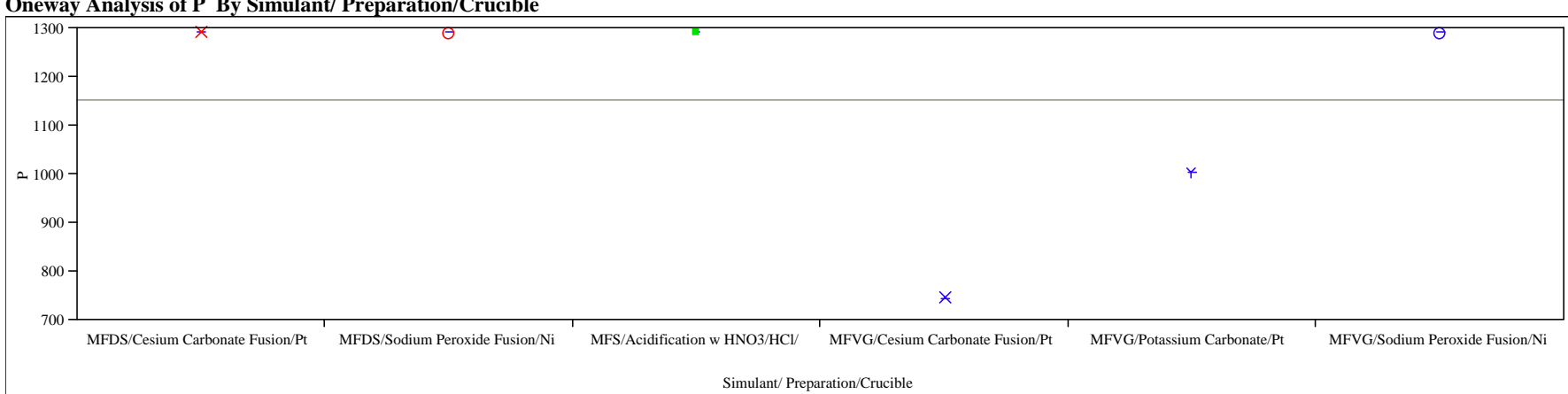

Oneway Anova

ummary of Fit

Rsquare

Adj Rsquare

Root Mean Square Error

Mean of Response

Observations (or Sum Wgts)

Analysis of Variance

Source

Simulant/ Preparation/Crucible

Error

C. Total

Means for Oneway Anova

Level

MFDS/Cesium Carbonate Fusion/Pt

MFS/Aodin Peroxide Fusin/N/

MFVG/C im Ca WHACl

MFVG/Cesium Carbonate Fusion/

MFVG/Sodium Pexhate

ST G/Sodium Peroxide Fusion/Ni

Preparation/Cruci

Means and Std Deviations

Level

MFDS/Cesium Carbonate Fusion/Pt

MFS/Aodifin Peroxide Fusion/N/

MFVG/Cision wNOB/HCl/

MFVG Cesium Carbonate Fusion

MFVG/Solim Peroxide Fusion

\begin{tabular}{|c|c|c|c|c|}
\hline DF & Sum of Squares & Mean Square & F Ratio & Prob $>$ F \\
\hline 5 & 263433.33 & 52686.7 & . & . \\
\hline 0 & 0.00 & & & \\
\hline 5 & 263433.33 & & & \\
\hline Number & Mean & Std Error & Lower 95\% & Upper 95\% \\
\hline 1 & 1290.00 & & . & . \\
\hline 1 & 1290.00 & & . & . \\
\hline 1 & 1290.00 & & . & . \\
\hline 1 & 745.00 & . & . & . \\
\hline 1 & 1005.00 & & . & . \\
\hline 1 & 1290.00 & . & . & . \\
\hline Number & Mean & Std Dev & Std Err Mean & Lower 95\% \\
\hline 1 & 1290.00 & & & \\
\hline 1 & 1290.00 & & . & \\
\hline 1 & 1290.00 & & . & \\
\hline 1 & 745.00 & . & . & \\
\hline 1 & 1005.00 & & . & \\
\hline 1 & 1290.00 & & . & \\
\hline
\end{tabular}

Upper 95\% 
WSRC-TR-2005-00166

SRNL-RPP-2005-00029

Revision 0

Exhibit A2. Statistical Comparisons of Preparation/Crucible Measurements by Element by Type of Sample

Oneway Analysis of Pb By Simulant/ Preparation/Crucible

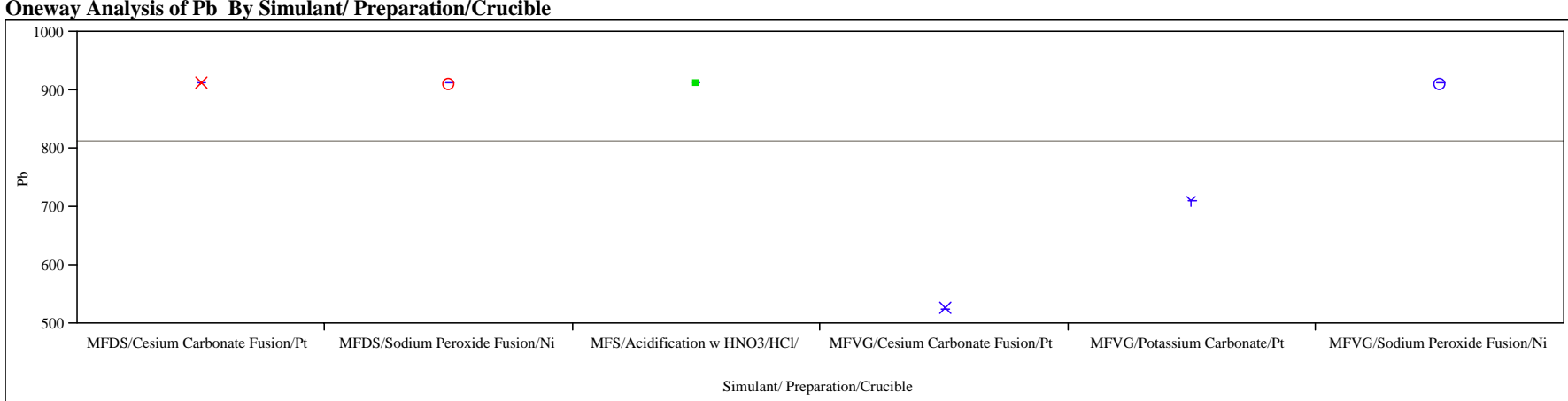

Oneway Anova

ummary of Fit

Rsquare

Adj Rsquare

Root Mean Square Error

Mean of Response

Observations (or Sum Wgts)

Analysis of Variance

Source

Simulant/ Preparation/Crucible

Error

Means for Oneway Anova

Level

MFDS/Cesium Carbonate Fusion/Pt

MFS/Aodidin Peroxide Fusion//

MFVG/Cesim Ca HNOB/HCl

MFVG/Cesium Carbonate Fusion/

MFVG/Sodium Peroxide Fusion

ST G/Sodium Peroxide Fusion/Ni

Preparation/Cruci

Means and Std Deviations

Level

MFDS/Cesium Carbonate Fusion/Pt

TFS/Aodun Peroxide Fusion/N/

MFVG/C i

MFVG Cesium Carbonate Fusiont

MFVG/Sodium Peroxide Fusion

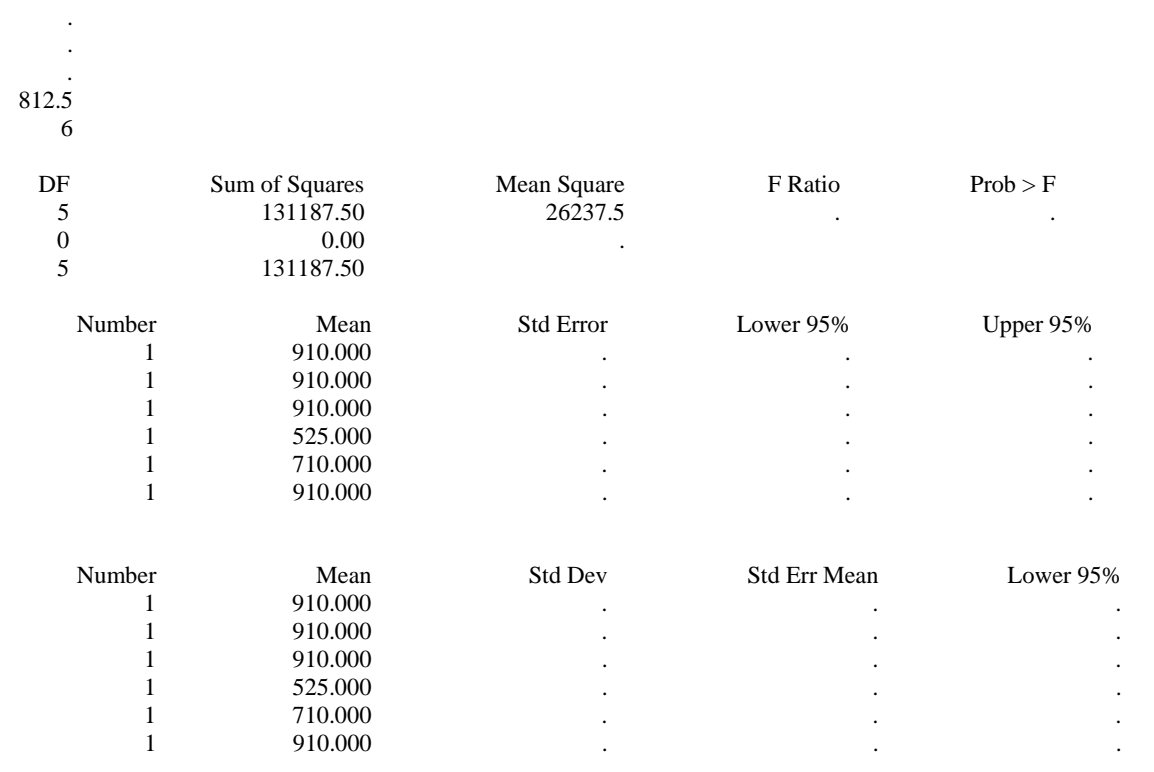

Upper 95\% 


\section{Exhibit A2. Statistical Comparisons of Preparation/Crucible Measurements by Element by Type of Sample}

Oneway Analysis of S By Simulant/ Preparation/Crucible

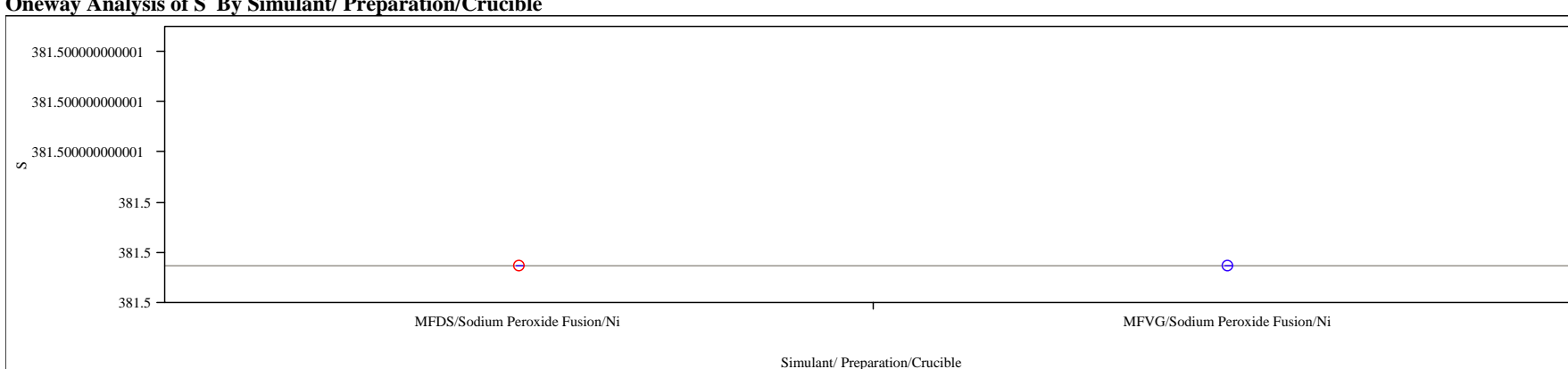

Missing Rows

Simulant/ Preparation/Crucible

4Oneway Anova

Summary of Fit

Rsquare

Adj Rsquare

Root Mean Square Erro

Mean of Response

Observations (or Sum Wgts)

MFDS/Sodium Peroxide Fusion/Ni-MFVG/Sodium Peroxide Fusion/N

Assuming equal variances

Difference

Std Err Dif

Upper CL Dif

Lower CL Dif

0 t Ratio

Prob $>|t|$

. Prob $>t$

Analysis of Variance

Source$$
0.95 \text { Prob }<\mathrm{t}
$$

Simulant/ Preparation/Crucible

Error

Means for Oneway Anova

Mear

MFDS/Sodium Peroxide Fusion/Ni



error variance

Means and Std Deviations

MFDS/Sodium Peroxide Fusion/N

MFVG/Sodium Peroxide Fusion/NI

1.0000
0.5000
0.500

0
.0000
5000
5000

\begin{tabular}{|c|c|c|c|c|c|}
\hline $\begin{array}{r}\text { DF } \\
1\end{array}$ & $\begin{array}{r}\text { Sum of Squares } \\
0\end{array}$ & $\begin{array}{r}\text { Mean Square } \\
0\end{array}$ & F Ratio & Prob $>$ F & \\
\hline 0 & 0 & . & & & \\
\hline 1 & 0 & & & & \\
\hline Number & Mean & Std Error & Lower 95\% & Upper 95\% & \\
\hline 1 & 381.500 & & 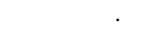 & & \\
\hline 1 & 381.500 & . & . & . & \\
\hline Number & Mean & Std Dev & Std Err Mean & Lower 95\% & Upper 95\% \\
\hline 1 & 381.500 & & & & \\
\hline 1 & 381.500 & & & & \\
\hline
\end{tabular}


WSRC-TR-2005-00166

SRNL-RPP-2005-00029

Revision 0

Exhibit A2. Statistical Comparisons of Preparation/Crucible Measurements by Element by Type of Sample

Oneway Analysis of Sb By Simulant/Preparation/Crucible

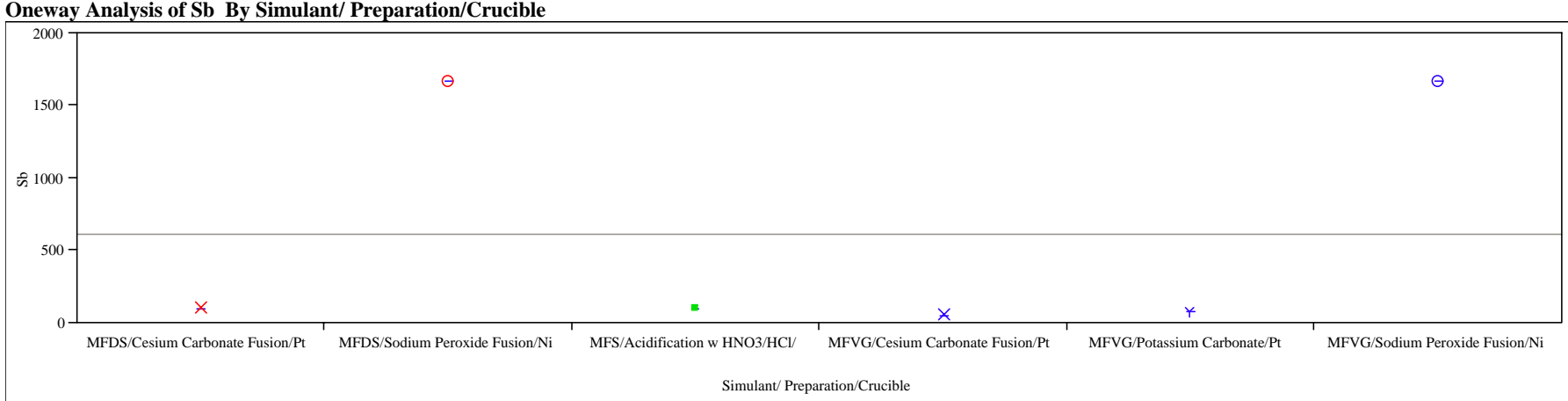

Oneway Anova

ummary of Fit

Rsquare

Adj Rsquare

Root Mean Square Error

Mean of Response

Observations (or Sum Wgts)

Analysis of Variance

Source

Simulant/ Preparation/Crucible

Error

Means for Oneway Anova

Level

MFDS/Cesium Carbonate Fusion/Pt

TFS/Aodidin Peroxide Fusion/Ni

MFVG/Cesiul CabNo3/HCl

MFVG/Cesium Carbonate Fusion/Pt

MFVG/Sodium Peabonater

MFision/Ni

Stror uniance

Means and Std Deviations

Level

MFDS/Cesium Carbonate Fusion/Pt

TFS/Aodum Peroxide Fusion/N/

MFVG/Cinit W WNOB/HCI

MFVG/Cotass Carbonate Fusion

MFVG/Solim Peroxide Fusior

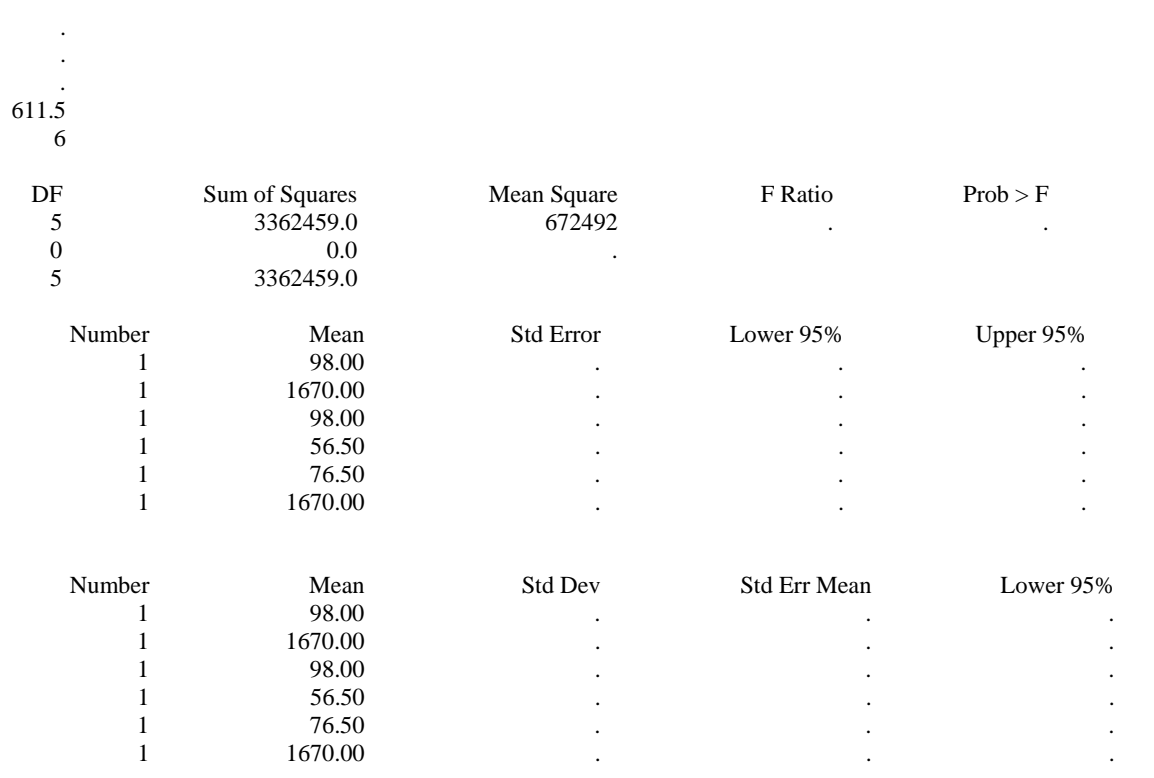

Upper 95\% 
WSRC-TR-2005-00166

SRNL-RPP-2005-00029

Revision 0

Exhibit A2. Statistical Comparisons of Preparation/Crucible Measurements by Element by Type of Sample

Oneway Analysis of Si By Simulant/ Preparation/Crucible

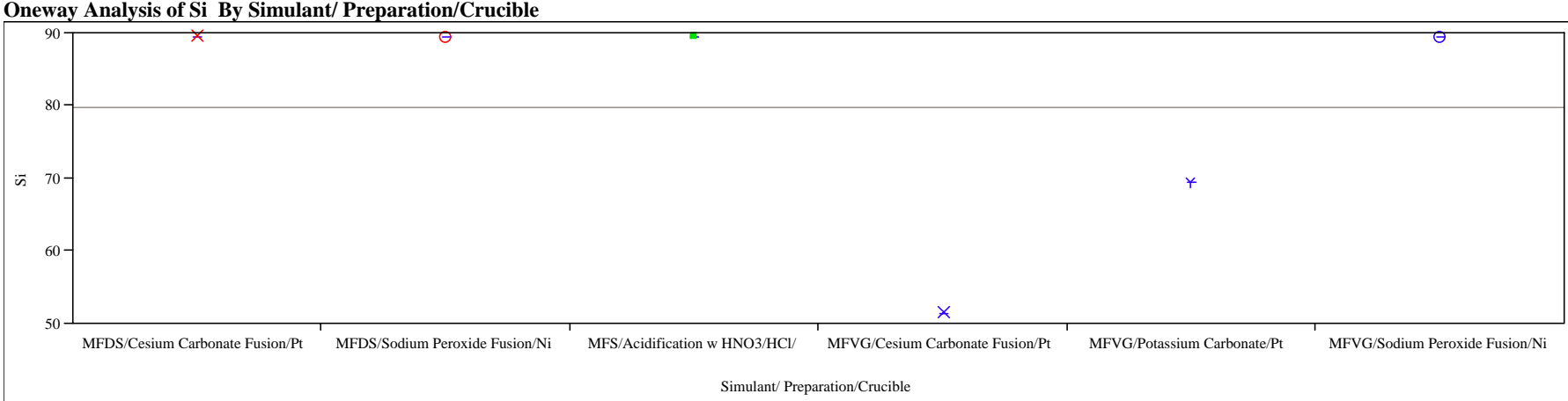

Oneway Anova

Summary of Fit

Rsquare

Adj Rsquare

Root Mean Square Erro

Mean of Response

Observations (or Sum Wgts)

Analysis of Variance

Source

Simulant/ Preparation/Crucible

Error

C. Total

MFDS/Cesium Carbonate Fusion/Pt

MFS/Sodim Peroxide Fusion/N/

MFVG/Ciction w HNO3/HCl/

MFVG/Cesium Carbonate Fusion/Pt

MFVG/Possim Carbonate/Pt

ST G/Sodium Peroxide Fusion/Ni

lant/ Preparation/Crucib

Means and Std Deviations

Level

FFDS/Cesium Carbonate Fusion/Pt

TFS/Acidicin Peroxide Fusion/Ny

MFVG/Cicaton w HNO3/HCl/

MFV/Cesium Carbonate Fusion

MFVG/Sodism Peroxide Fusiont

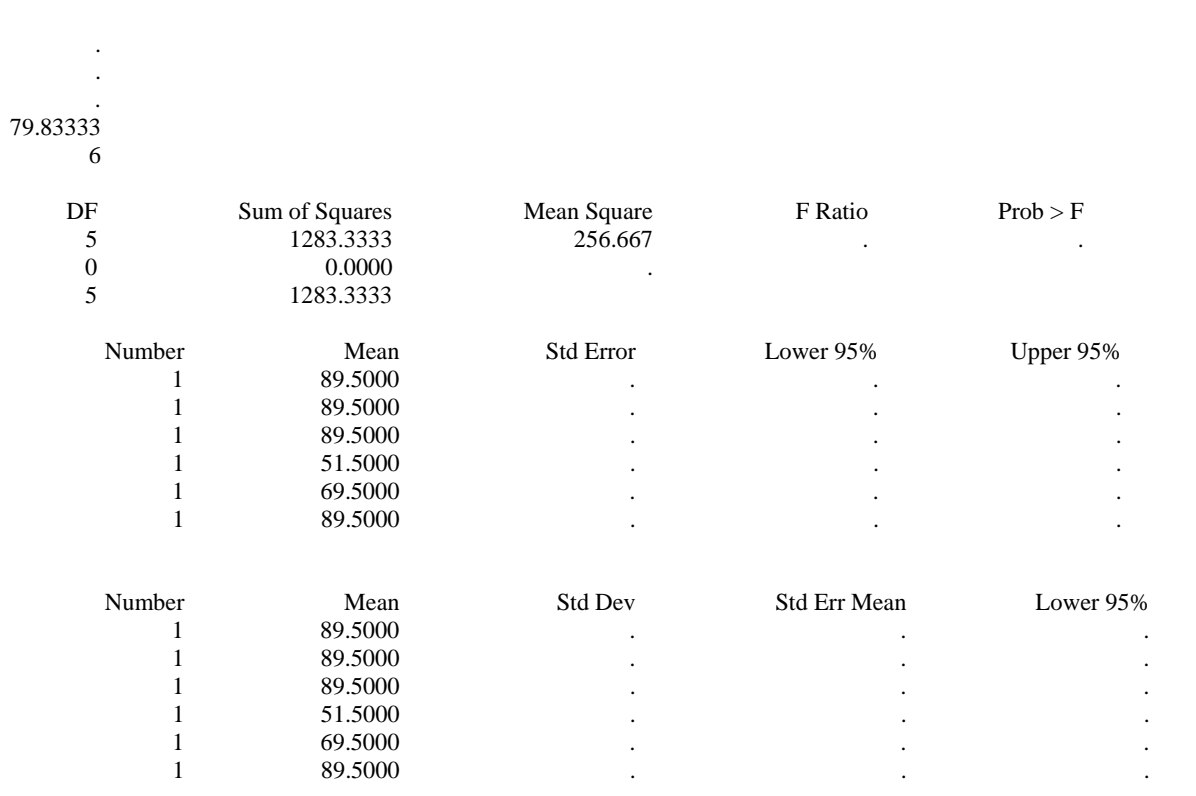

Upper 95\% 
WSRC-TR-2005-00166

SRNL-RPP-2005-00029

Revision 0

Exhibit A2. Statistical Comparisons of Preparation/Crucible Measurements by Element by Type of Sample

Oneway Analysis of Sn By Simulant/Preparation/Crucible

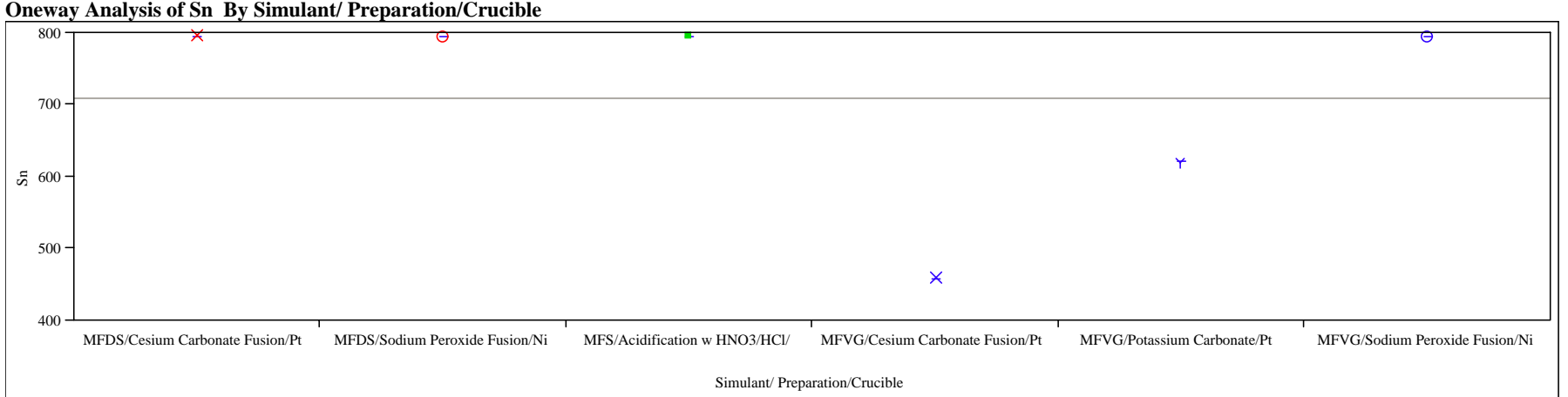

Oneway Anova

Summary of Fit

Rsquare

Adj Rsquare

Root Mean Square Erro

Mean of Response

Observations (or Sum Wgts)

Analysis of Variance

Source

Simulant/ Preparation/Crucible

Error

C. Total

MFDS/Cesium Carbonate Fusion/Pt

MFS/Sodim Peroxide Fusion/N/

FS/Acidification w HNO3/HCl/

MFVG/Cesium Carbonate Fusion/

MFVG/Sossinn Carbonate/Pr

Stention

nt/Preparation/Crucible

Means and Std Deviations

Level

FDDS/Cesium Carbonate Fusion/Pt

FF/Acidin Pexide Fusion/Ni

MFVG/Cicaton w HNO3/HCl/

MFVG Cosium Carbonate Fusion

MFVG/Sodium Peroxide Fusion

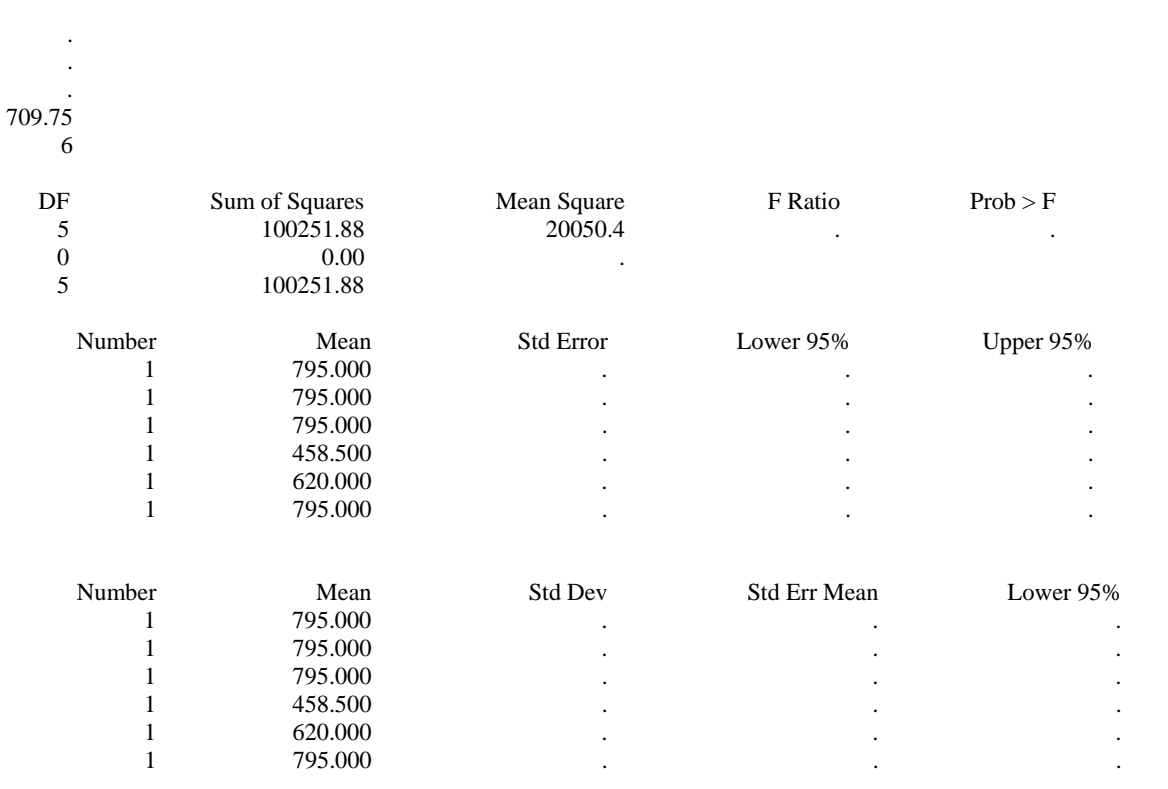

Upper 95\% 
WSRC-TR-2005-00166

SRNL-RPP-2005-00029

Revision 0

Exhibit A2. Statistical Comparisons of Preparation/Crucible Measurements by Element by Type of Sample

Oneway Analysis of Sr By Simulant/ Preparation/Crucible

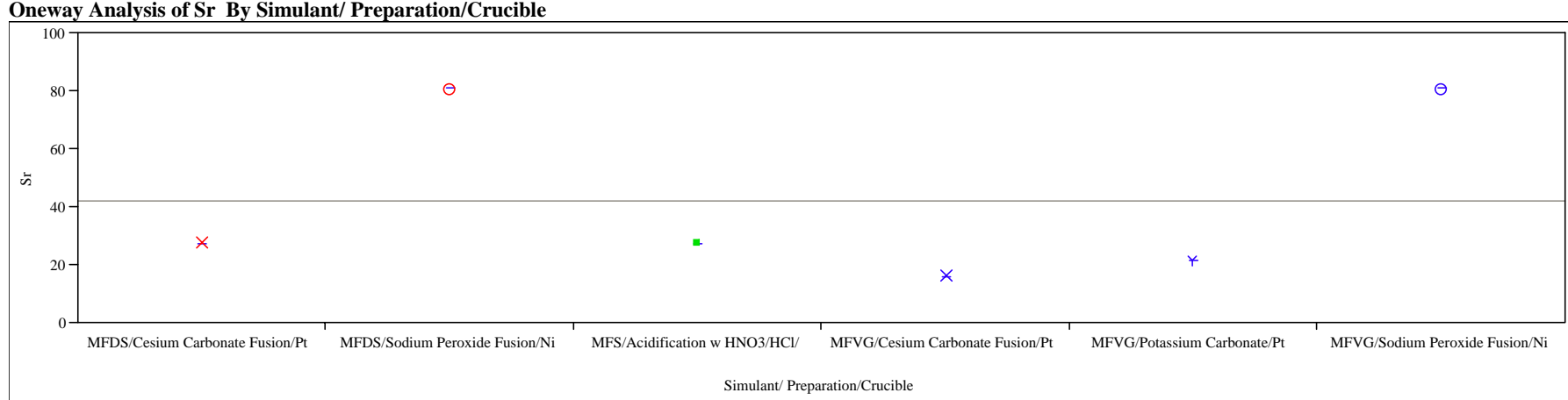

Oneway Anova

ummary of Fit

Rsquare

Adj Rsquare

Root Mean Square Erro

Mean of Response

Observations (or Sum Wgts)

Analysis of Variance

Source

Simulant/ Preparation/Crucible

Error

Means for Oneway Anova

MFDS/Cesium Carbonate Fusion/Pt

MFS/Sodium Peroxide Fusion/Ni

MFVG/Cicion w HNO3/HCl/

MFVG/Cesium Carbonate Fusion/

MFVG/Potassilum Carbonate/Pt

Serion $/ \mathrm{Ni}$

lant/ Preparation/Crucible

Means and Std Deviations

Level

MFDS/Cesium Carbonate Fusion/Pt

MS/Aolim Peroxide Fusion/Ni

MFVG/Cicaton w HNO3/HCl/

MFV/Cesium Carbonate Fusion

MFVG/Sodium Perbide Fusion

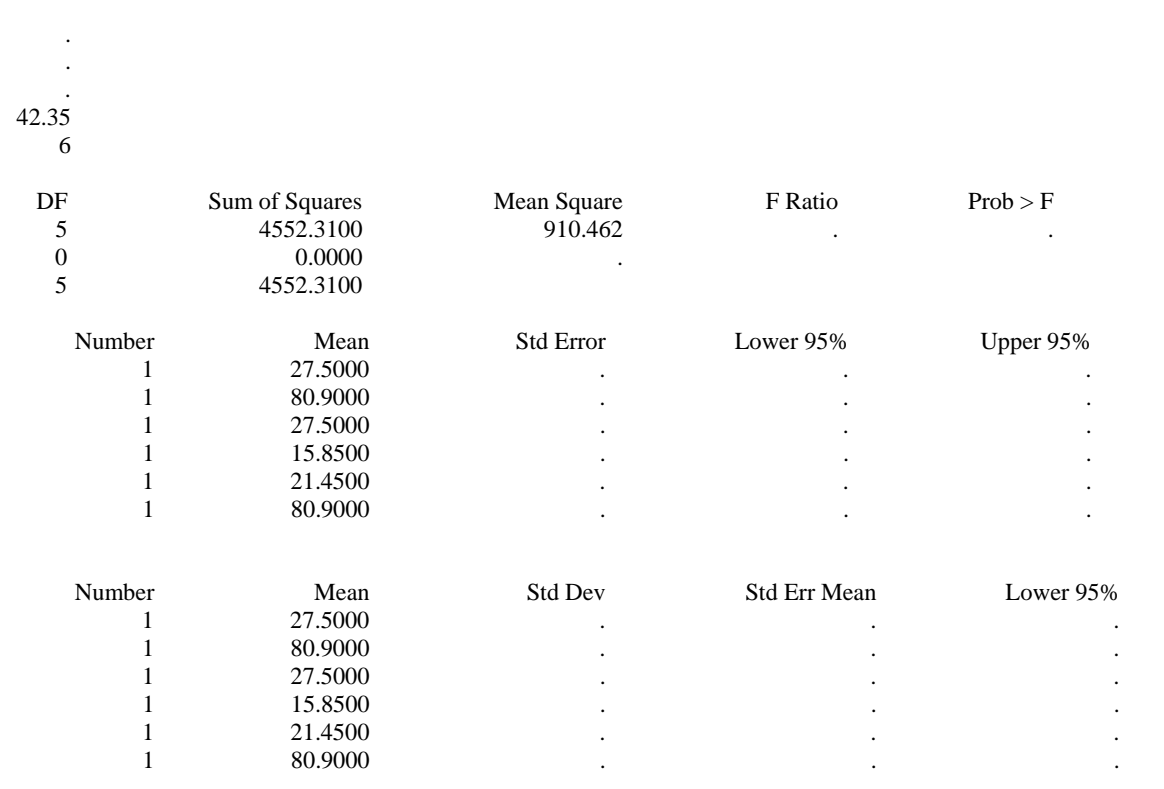

Upper 95\% 


\section{Exhibit A2. Statistical Comparisons of Preparation/Crucible Measurements by Element by Type of Sample}

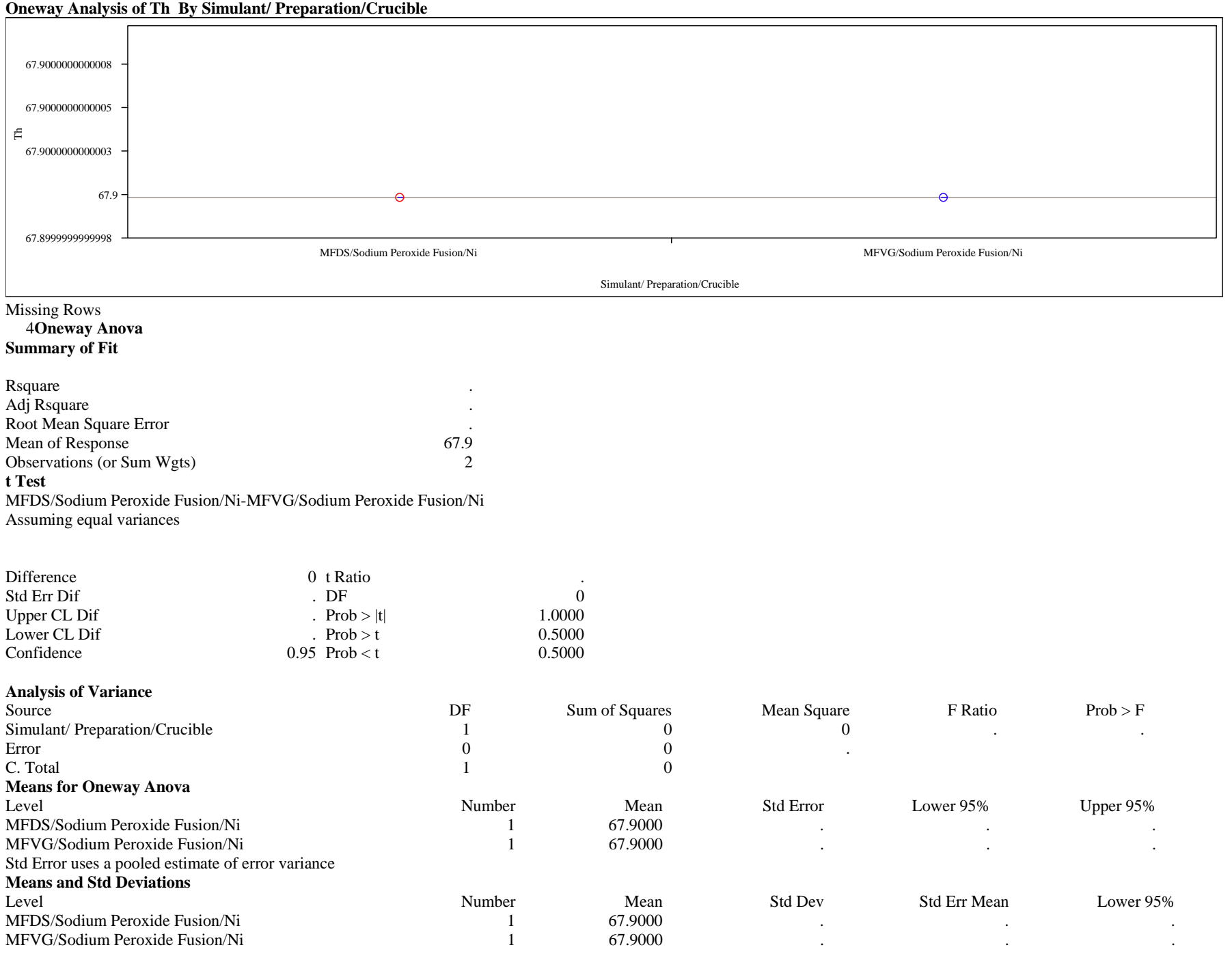


WSRC-TR-2005-00166

SRNL-RPP-2005-00029

Revision 0

Exhibit A2. Statistical Comparisons of Preparation/Crucible Measurements by Element by Type of Sample

Oneway Analysis of Ti By Simulant/ Preparation/Crucible

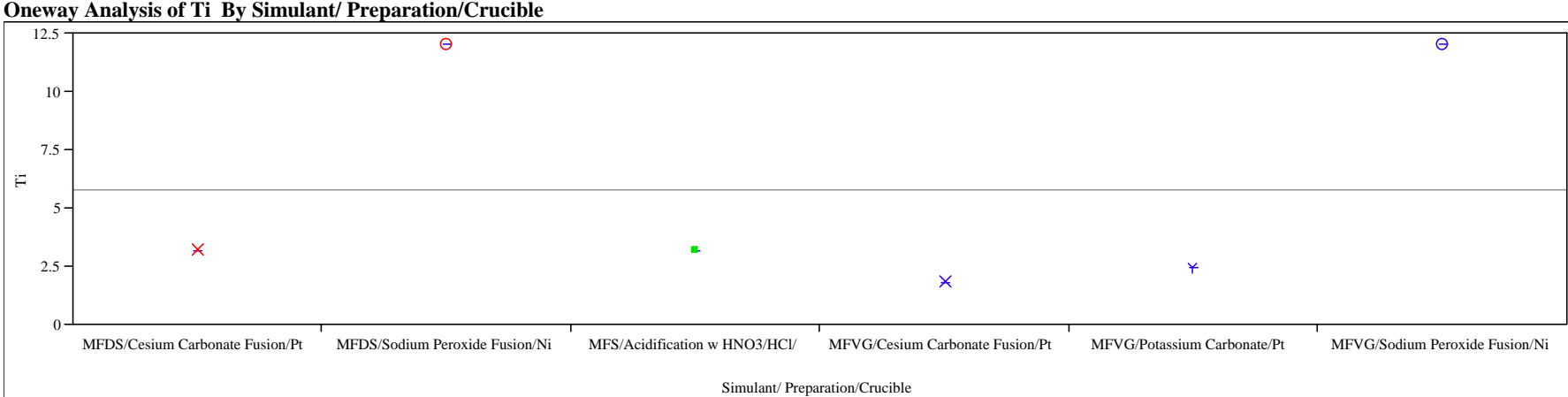

Oneway Anova

Summary of Fit

Rsquare

Adj Rsquare

Root Mean Square Erro

Mean of Response

Observations (or Sum Wgts)

Analysis of Variance

Source

Simulant/ Preparation/Crucible

Error

Means for Oneway Anova

Level

MFDS/Cesium Carbonate Fusion/Pt

TFS/Aodum Peroxide Fusion/Ni/

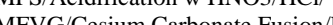

MFVG/Cesium Carbonate Fusion/Pt

MFVG/Sodium Pabost

MFV/Sodium Peroxide Fusion/Ni

/ Preparation/Cruc

Means and Std Deviations

Level

MFDS/Cesium Carbonate Fusion/Pt

MFS/Sodum Peroxide Fusion/Ni

MFVG/Cesim W HNO3/HCl/

MFVG Cesium Carbonate Fusion

MFVG/Sodium Peroxide Fusion

\begin{tabular}{|c|c|c|c|c|}
\hline DF & Sum of Squares & Mean Square & F Ratio & Prob $>$ F \\
\hline 5 & 119.02532 & 23.8051 & . & . \\
\hline 0 & 0.00000 & & & \\
\hline 5 & 119.02532 & & & \\
\hline Number & Mean & Std Error & Lower 95\% & Upper 95\% \\
\hline 1 & 3.1600 & . & . & Prentos. \\
\hline 1 & 12.0500 & . & . & . \\
\hline 1 & 3.1600 & . & . & . \\
\hline 1 & 1.8200 & . & . & . \\
\hline 1 & 2.4650 & . & . & . \\
\hline 1 & 12.0500 & . & . & . \\
\hline Number & Mean & Std Dev & Std Err Mean & Lower 95\% \\
\hline 1 & 3.1600 & & & \\
\hline 1 & 12.0500 & . & . & . \\
\hline 1 & 3.1600 & . & . & . \\
\hline 1 & 1.8200 & . & . & \\
\hline 1 & 2.4650 & . & . & . \\
\hline 1 & 12.0500 & . & . & \\
\hline
\end{tabular}

Upper 95\% 


\section{Exhibit A2. Statistical Comparisons of Preparation/Crucible Measurements by Element by Type of Sample}

Oneway Analysis of Tl By Simulant/Preparation/Crucible

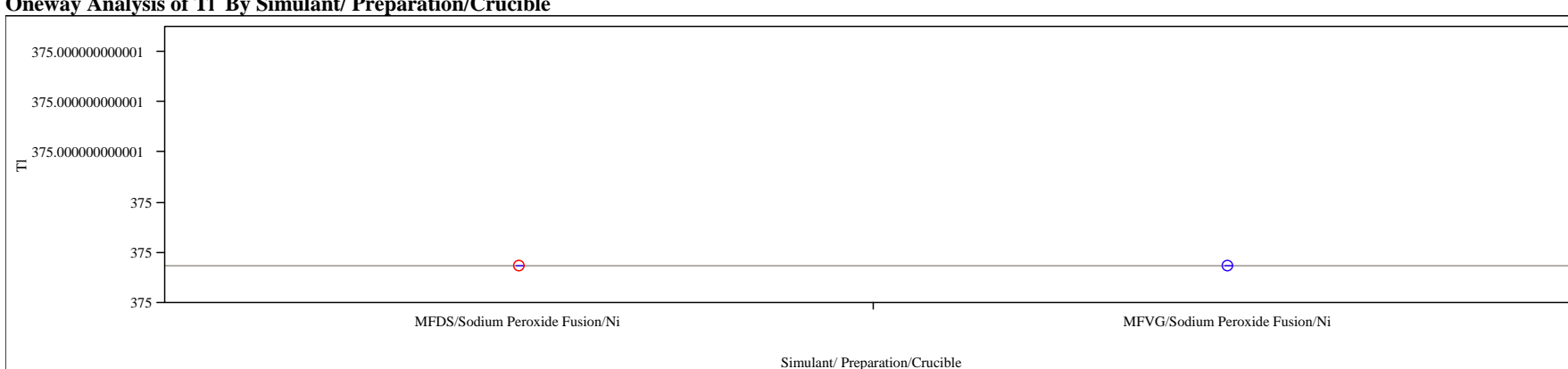

Missing Rows

Simulant/ Preparation/Crucible

Oneway Anov

Summary of Fit

Rsquare

Adj Rsquare

Root Mean Square Erro

Mean of Response

Observations (or Sum Wgts)

Test

MFDS/Sodium Peroxide Fusion/Ni-MFVG/Sodium Peroxide Fusion/N

Assuming equal variances

Difference

Std Err Dif

Upper CL Dif

Lower CL Dif

0 t Ratio

Prob $>|t|$

. Prob $>t$

0.5000

0.95 Prob $<$

5000

Analysis of Variance

Source

Simulant/ Preparation/Crucible

Error

C. Total

Means for Oneway Anova

Level

MFDS/Sodium Peroxide Fusion/N

MFV

Steror uses a pooled estinate of error variance

Means and Std Deviations

MFDS/Sodium Peroxide Fusion/N

MFVG/Sodium Peroxide Fusion/N

$\begin{array}{rrrrr}\text { DF } & \text { Sum of Squares } & \text { Mean Square } & \text { F Ratio } & \text { Prob > F } \\ 1 & 0 & 0 & & \\ 0 & 0 & & & \\ 1 & 0 & & & \\ & & & & \\ \text { Number } & \text { Mean } & \text { Std Error } & \text { Lower 95\% } & \text { Upper 95\% } \\ 1 & 375.000 & . & . & . \\ 1 & 375.000 & . & & \\ & & & & \text { Lower 95\% } \\ & \text { Mean } & \text { Std Dev } & \text { Std Err Mean } & .\end{array}$

Upper 95\% 


\section{Exhibit A2. Statistical Comparisons of Preparation/Crucible Measurements by Element by Type of Sample}

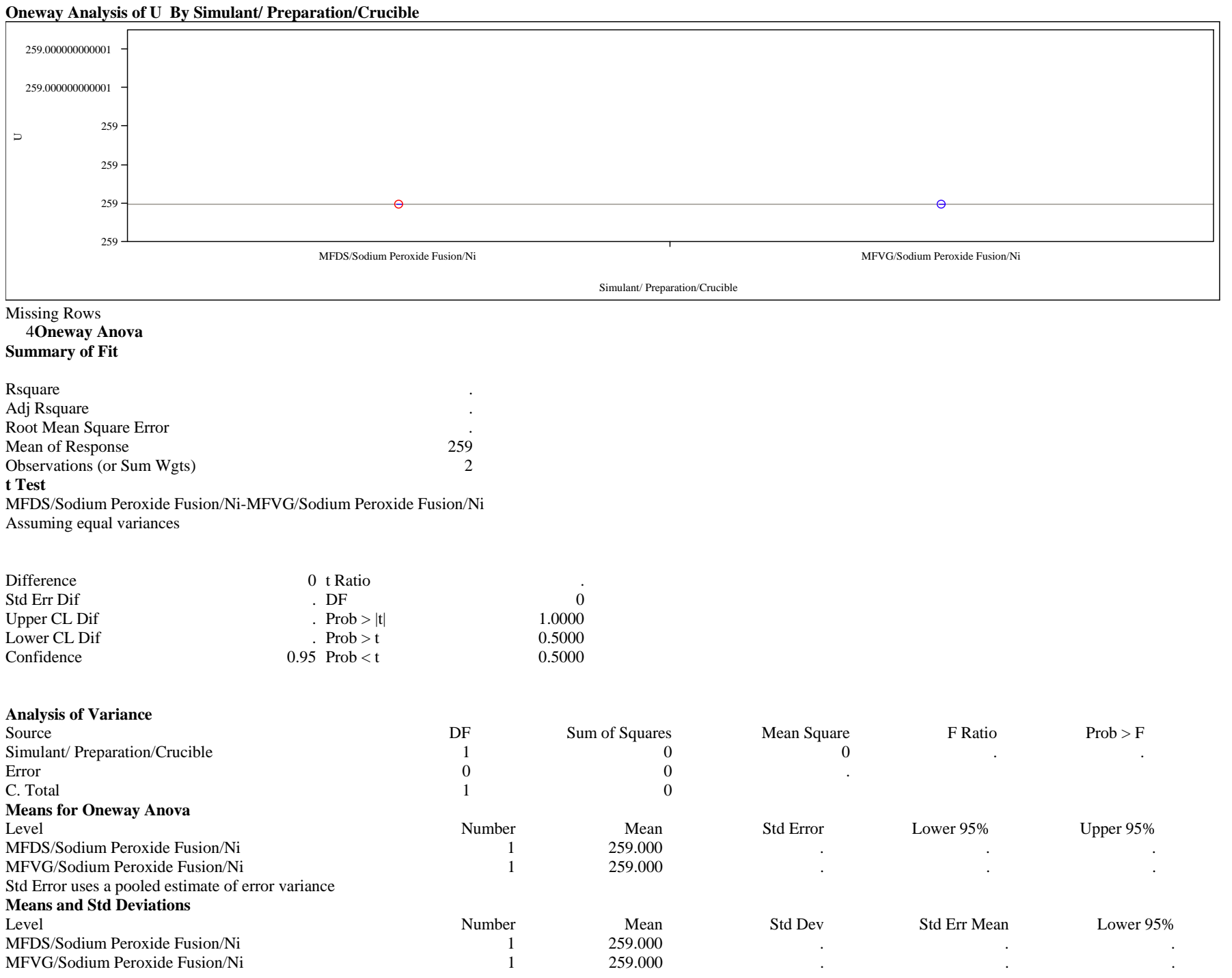


WSRC-TR-2005-00166

SRNL-RPP-2005-00029

Revision 0

Exhibit A2. Statistical Comparisons of Preparation/Crucible Measurements by Element by Type of Sample

Oneway Analysis of Y By Simulant/ Preparation/Crucible

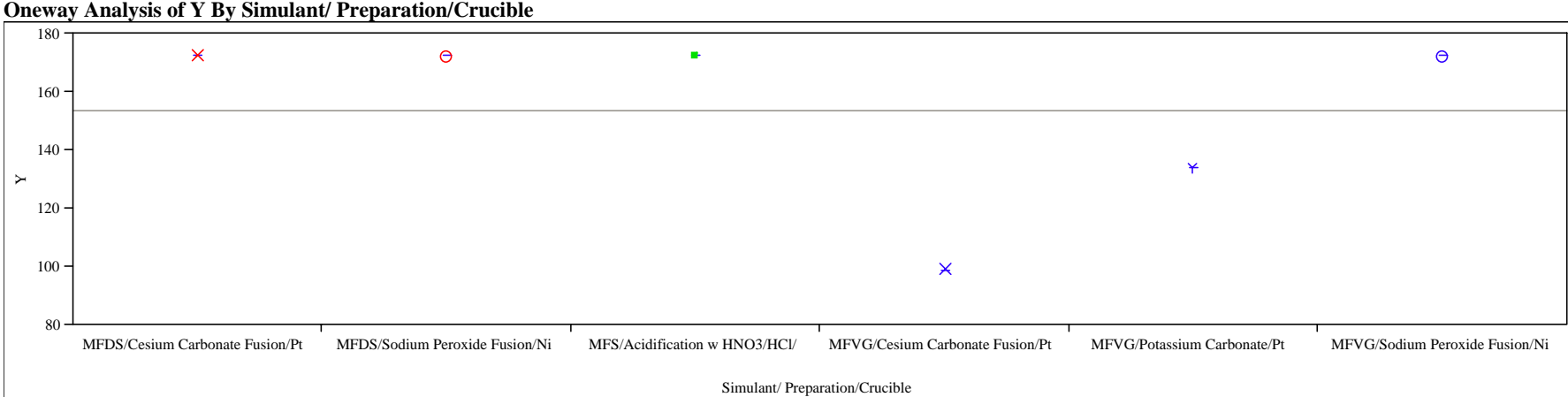

Oneway Anova

Simulant/Preparation/Crucible

ummary of Fit

Rsquare

Adj Rsquare

Root Mean Square Error

Mean of Response

Observations (or Sum Wgts)

Analysis of Variance

Source

Simulant/ Preparation/Crucible

Error

Means for Oneway Anova

Level

MFDS/Cesium Carbonate Fusion/Pt

MFS/Acidifiction w

MFVG/Cesiul Ca HNOB/HCl/

MFVG/Cesium Carbonate Fusion/Pt

MFVG/Sodium Paboste

ST/Sodium Peroide Fusion/Ni

Std Erisor uses a poever error variance

Means and Std Deviation

MFDel

MFDS/Cesium Carbonate Fusion/Pt

TFS/Aodum Peroxide Fusion/N/

MFVG/Cinit $\mathrm{W}$ HNO3/HCl/

MFVG/Potass Carbonate Fusion/Pt

MFVG/Solim Peroxide Fusiont

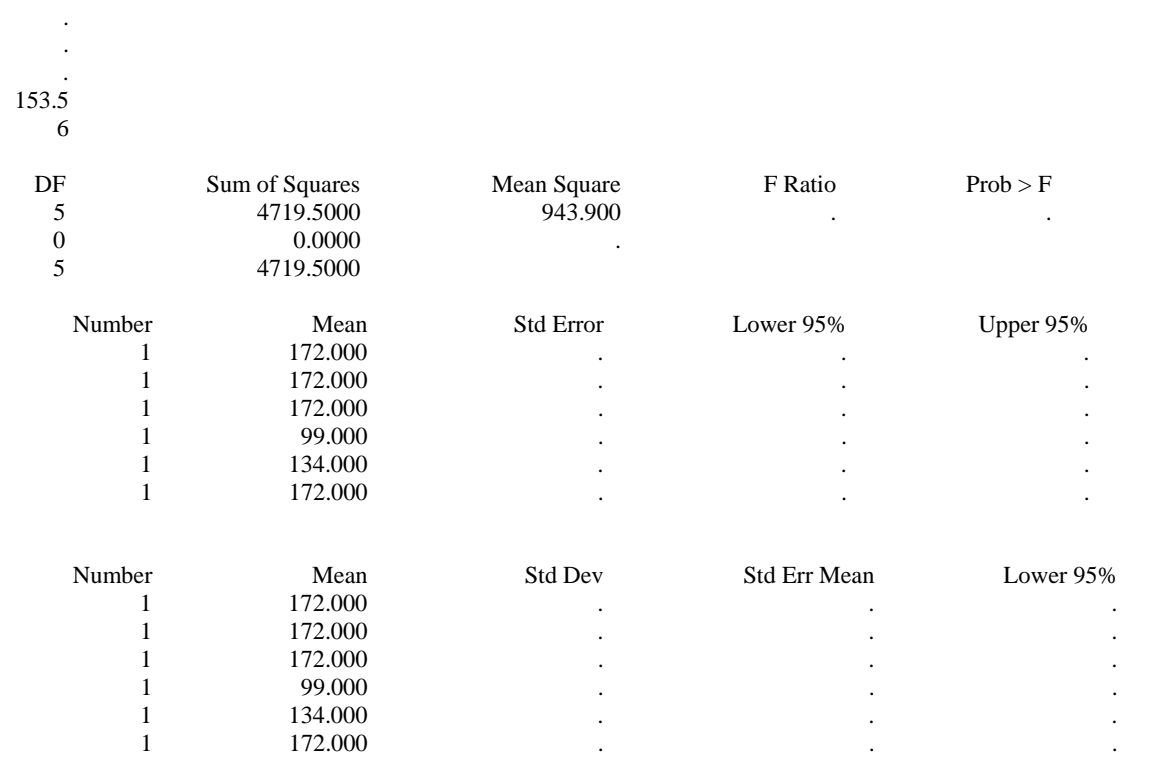

Upper 95\% 
WSRC-TR-2005-00166

SRNL-RPP-2005-00029

Revision 0

Exhibit A2. Statistical Comparisons of Preparation/Crucible Measurements by Element by Type of Sample

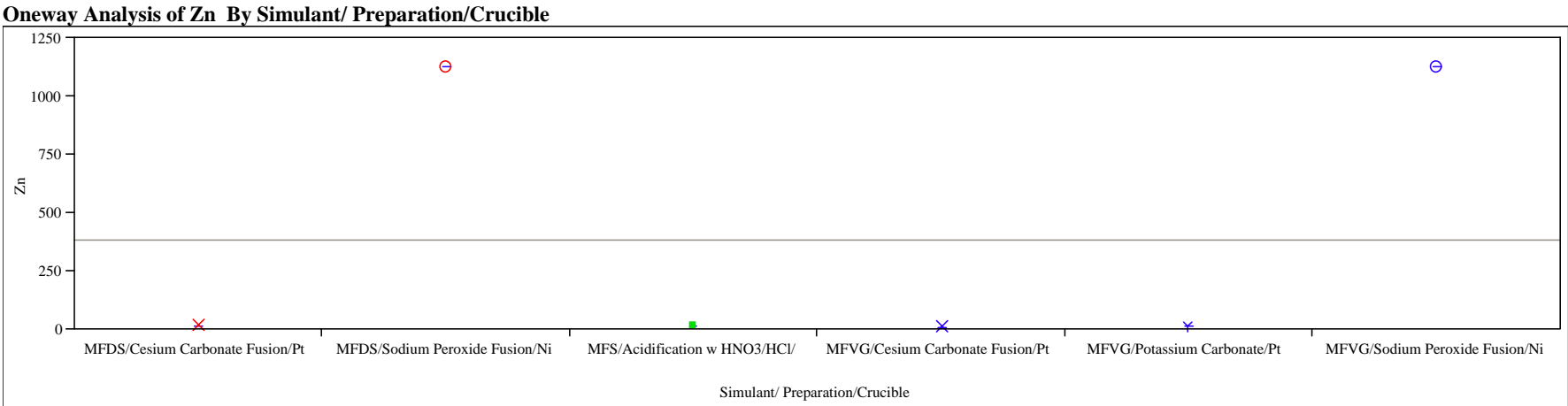

Oneway Anova

Summary of Fit

Rsquare

Adj Rsquare

Root Mean Square Erro

Mean of Response

Observations (or Sum Wgts)

Analysis of Variance

Source

imulant/ Preparation/Crucible

Error

Means for Oneway Anova

Level

MFDS/Cesium Carbonate Fusion/Pt

MFS/Aodum Peroxide Fusion/N/

MFVG/C i Caton WNOB/HCI/

MFVG/Cesium Carbonate Fusion/Pt

MFVG/Sodium Paboste

MFVG/Sodium Peroide Fusion/Ni

/ Preparation/Crucil

Means and Std Deviations

Level

MFDS/Cesium Carbonate Fusion/Pt

TFS/Aodum Peroxide Fusion/N/

MFVG/Cisit $\mathrm{W}$ HNO3/HCl/

MFVG Cesium Carbonate Fusion

MFVG/Sodium Peroxide Fusion

\begin{tabular}{|c|c|c|c|c|}
\hline $\mathrm{DF}$ & Sum of Squares & Mean Square & F Ratio & Prob $>$ F \\
\hline 5 & 1659380 & 331876 & . & . \\
\hline 0 & $-2.328 \mathrm{e}-10$ & & & \\
\hline 5 & 1659380 & & & \\
\hline Number & Mean & Std Error & Lower 95\% & Upper 95\% \\
\hline 1 & 17.20 & . & . & . \\
\hline 1 & 1130.00 & . & . & . \\
\hline 1 & 17.20 & . & . & . \\
\hline 1 & 9.90 & . & . & . \\
\hline 1 & 13.40 & . & . & . \\
\hline 1 & 1130.00 & . & . & . \\
\hline Number & Mean & Std Dev & Std Err Mean & Lower 95\% \\
\hline 1 & 17.20 & & & \\
\hline 1 & 1130.00 & . & . & . \\
\hline 1 & 17.20 & . & . & . \\
\hline 1 & 9.90 & . & . & . \\
\hline 1 & 13.40 & . & . & . \\
\hline 1 & 1130.00 & . & . & . \\
\hline
\end{tabular}

Upper 95\% 
WSRC-TR-2005-00166

SRNL-RPP-2005-00029

Revision 0

Exhibit A2. Statistical Comparisons of Preparation/Crucible Measurements by Element by Type of Sample

Oneway Analysis of Zr By Simulant/ Preparation/Crucible

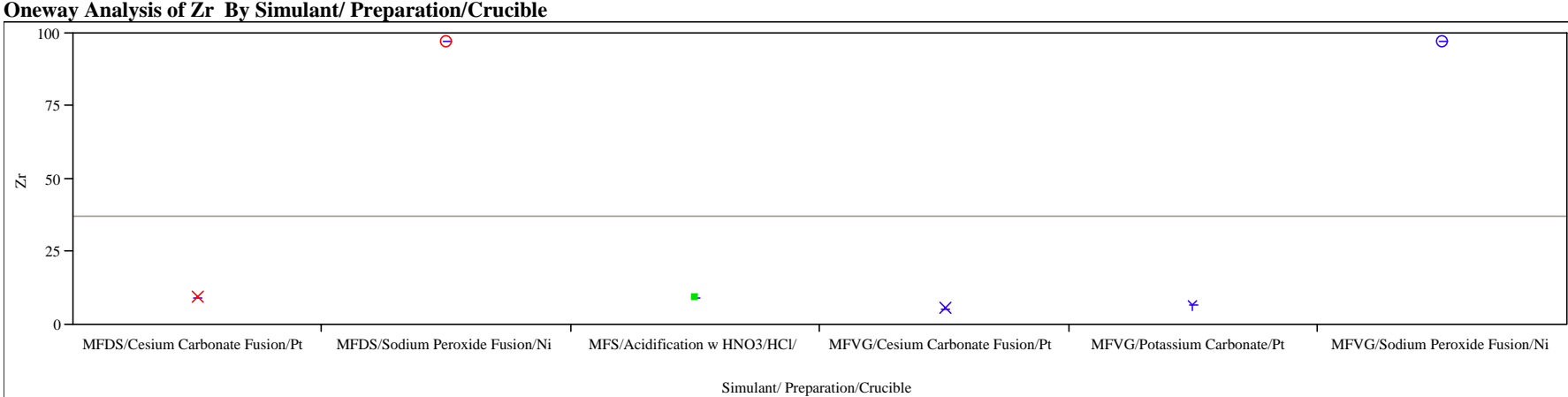

Oneway Anova

Simulant/Preparation/Crucible

ummary of Fi

Rsquare

Adj Rsquare

Root Mean Square Error

Mean of Response

Observations (or Sum Wgts)

Analysis of Variance

Source

Simulant/ Preparation/Crucible

Error

Means for Oneway Anova

Level

MFDS/Cesium Carbonate Fusion/Pt

MFS/Aodin Peroxide Fusin/N/

MFVG/C i Ca W

MFVG/Cesium Carbonate Fusion/Pt

MFVG/Sodium Pexbonatert

ST/Sodium Peroide Fusion/Ni

Stror uniance

Means and Std Deviatio

Level

MFDS/Cesium Carbonate Fusion/Pt

MFS/Sodum Peroxide Fusion/Ni

MFVG/Cision wNOB/HCl/

MFVG Cesium Carbonate Fusion

MFVG/Solium Peroxide Fusion

\begin{tabular}{|c|c|c|c|c|}
\hline DF & Sum of Squares & Mean Square & F Ratio & Prob $>$ F \\
\hline 5 & 10699.847 & 2139.97 & . & . \\
\hline 0 & 0.000 & & & \\
\hline 5 & 10699.847 & & & \\
\hline Number & Mean & Std Error & Lower 95\% & Upper 95\% \\
\hline 1 & 9.1500 & . & . & . \\
\hline 1 & 97.2000 & . & . & . \\
\hline 1 & 9.1500 & . & . & . \\
\hline 1 & 5.2500 & . & . & . \\
\hline 1 & 7.1000 & . & . & . \\
\hline 1 & 97.2000 & . & . & . \\
\hline Number & Mean & Std Dev & Std Err Mean & Lower 95\% \\
\hline 1 & 9.1500 & . & . & 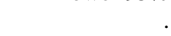 \\
\hline 1 & 97.2000 & . & . & . \\
\hline 1 & 9.1500 & . & . & \\
\hline 1 & 5.2500 & . & . & . \\
\hline 1 & 7.1000 & . & . & \\
\hline 1 & 97.2000 & . & . & . \\
\hline
\end{tabular}

Upper 95\% 
WSRC-TR-2005-00166

SRNL-RPP-2005-00029

Revision 0

\section{Exhibit A2. Statistical Comparisons of Preparation/Crucible Measurements by Element by Type of Sample}

Type of Sample=LCS

Oneway Analysis of Ag By Simulant/Preparation/Crucible

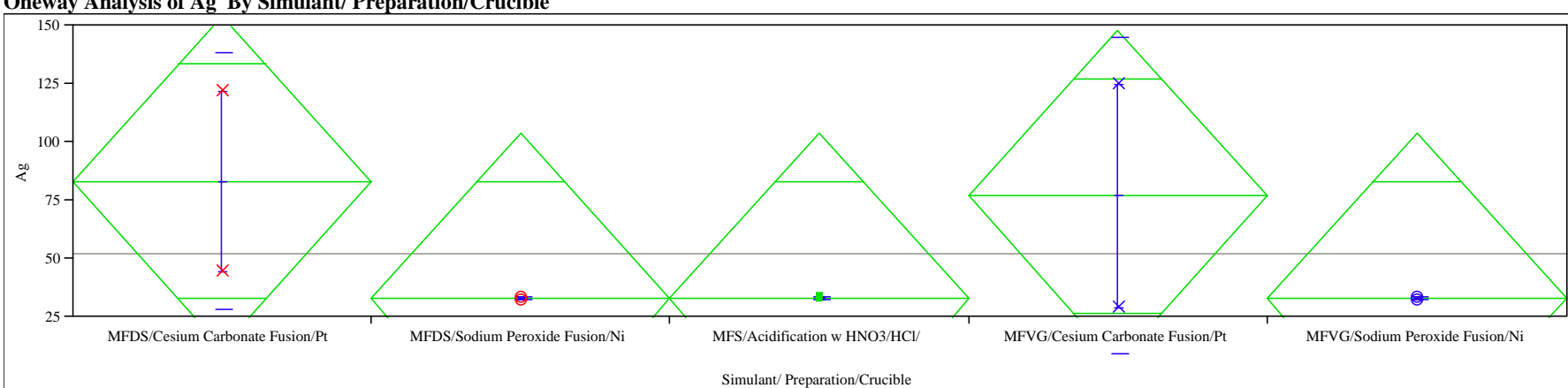

Oneway Anova

Summary of Fit

Rsquare

Adj Rsquare

Root Mean Square

Mean of Response

Analysis of Variance

Source

Simulant/ Preparation/Crucible

Error

Means for Oneway Anova

Means

MFDS/Cesium Carbonate Fusion/Pt

MFS/Aodicin Peroxide Fusion/M/

MFVG/Cesium W WNOB/HCI/

MFVG/Sodium Peroxide Fusion/Pt

ST/Sodiun Peroxide Fusion/Ni

variance

Means and Std Deviations

Level

MFDS/Cesium Carbonate Fusion/Pt

MFS/Aodum Peroxide Fusion/N/

MFVG/C i C W HNOB/

MFVG/Sodin Carbonate Fusion/Pt

0.411743

39.08518

51.835

835
10

DF
4
5
9

5346.304

7638.256

Number

Mean
83.1750
33.0250
33.0250
76.9250
33.0250

1336.58

F Ratio

Prob $>$ F

27.637
27.637

27.637

27.637
27.637

27.637
27.637

Number

Mean
83.1750
33.0250
33.0250
76.9250
33.0250

Std Dev
54.9068

54.9068
0.6010

0.6010

0.6010

$\begin{array}{rr}33.0250 & 0.6010\end{array}$

$\begin{array}{rr}\text { Lower 95\% } & \text { Upper 95\% } \\ 12.13 & 154.22 \\ -38.02 & 104.07 \\ -38.02 & 104.07 \\ 5.88 & 147.97 \\ -38.02 & 104.07 \\ & \\ & \\ \text { Std Err Mean } & \text { Lower } 95 \% \\ 38.825 & -410.1 \\ 0.425 & 27.6 \\ 0.425 & 27.6 \\ 48.075 & -533.9 \\ 0.425 & 27.6\end{array}$

Upper $95 \%$

576.49

38.43

387.78
38.43 
WSRC-TR-2005-00166

SRNL-RPP-2005-00029

Revision 0

Exhibit A2. Statistical Comparisons of Preparation/Crucible Measurements by Element by Type of Sample

Oneway Analysis of Al By Simulant/Preparation/Crucible

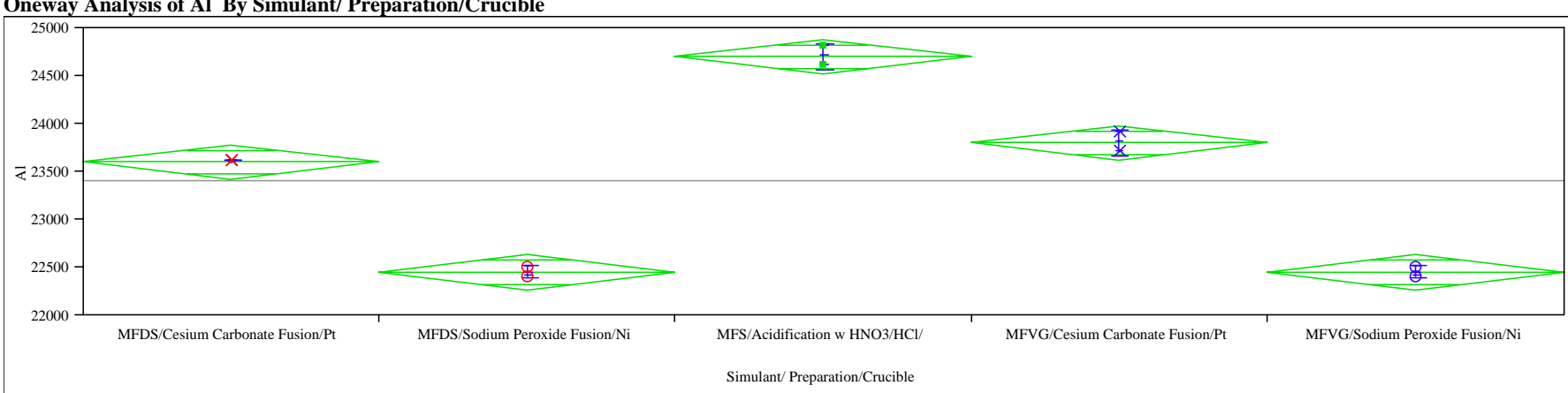

Oneway Anova

Summary of Fit

Rsquare

Adj Rsquare

Root Mean Square Erro

Mean of Response

Observations (or Sum Wgts)

Analysis of Variance

Simulant/Preparation/Crucible

C. Total

C. Toans for Oneway Anova

Level

MFDS/Cesium Carbonate Fusion/Pt

MFDS/Sodium Peroxide Fusion/Ni

MFS/Acidification w $\mathrm{HNO} / \mathrm{HCl} /$

MFVG/Cesium Carbonate Fusion/Pt

Std Error uses a pooled estimate of error variance

Means and Std Deviations

Level

MFDS/Cesium Carbonate Fusion/Pt

MFDS/Sodium Peroxide Fusion/Ni

MFS/Acidification w $\mathrm{HNO} / \mathrm{HCl}$

MFVG/Cesium Carbonate Fusion/Pt

MFVG/Sodium Peroxide Fusion/Ni
0.99328

0.987903

100

10

DF
4
5
9

Sum of Squares

7390000.0

$\begin{array}{rr}5 & 50000.0 \\ 9 & 7440000.0\end{array}$

Mean Square

1847500

F Ratio

Prob $>$ F

Number

$$
\begin{array}{r}
\text { Mean } \\
23600.0
\end{array}
$$

23600.0

22450.0

24700.0

23800.0

Std Error

70.711
70.711

70.711
70.711

70.711
70.711

70.711

$\begin{array}{rr}\text { Mean } & \text { Std Dev } \\ 23600.0 & 0.000\end{array}$

23600.0

22450.0

24700.0

23800.0

70.711

141.421

141.421
70.711

$\begin{array}{rr}23418 & 23782 \\ 22268 & 22632 \\ 24518 & 24882 \\ 23618 & 23982 \\ 22268 & 22632 \\ & \\ \text { Std Err Mean } & \text { Lower } 95 \% \\ 0.00 & 23600 \\ 50.00 & 21815 \\ 100.00 & 23429 \\ 100.00 & 22529 \\ 50.00 & 21815\end{array}$

Upper 95\%

23600
23085

23085

25071 
WSRC-TR-2005-00166

SRNL-RPP-2005-00029

Revision 0

Exhibit A2. Statistical Comparisons of Preparation/Crucible Measurements by Element by Type of Sample

Oneway Analysis of B By Simulant/Preparation/Crucible

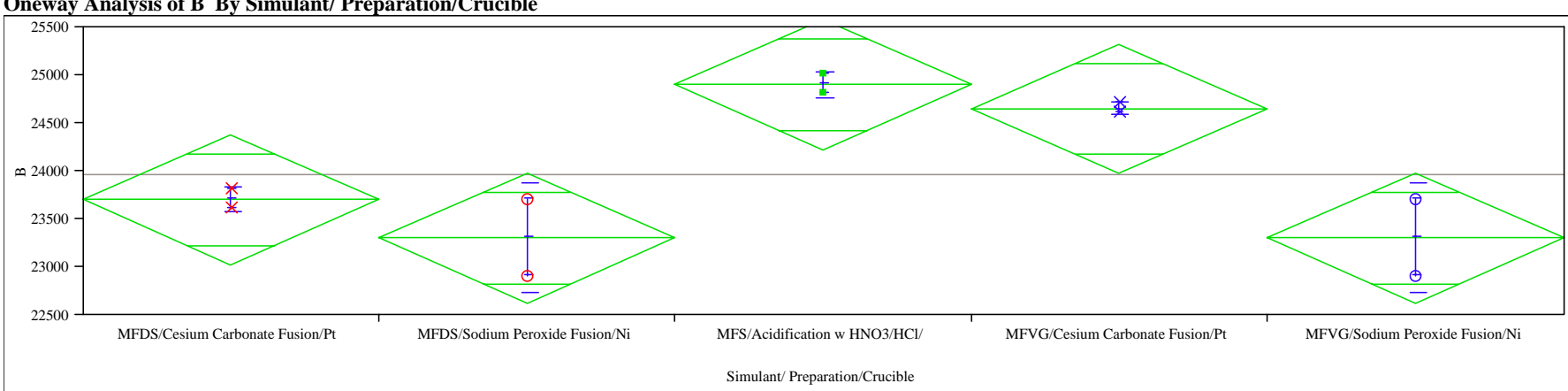

Oneway Anova

Summary of Fit

Rsquare

Adj Rsquare

Root Mean Square Erro

Mean of Response

Observations (or Sum Wgts)

Analysis of Variance

Simulant/Preparation/Crucible

C. Total

Level

MFDS/Cesium Carbonate Fusion/Pt

MFDS/Sodium Peroxide Fusion/Ni

MFS/Acidification w $\mathrm{HNO} / \mathrm{HCl} /$

MFVG/Cesium Carbonate Fusion/Pt

odium Peroxide Fusion/N

Std Error uses a pooled estimate of error variance

Means and Std Deviations

Level

MFDS/Cesium Carbonate Fusion/Pt

MFDS/Sodium Peroxide Fusion/Ni

MFS/Acidification w HNO3/HCl/

MFVG/Cesium Carbonate Fusion/Pt

MFVG/Sodium Peroxide Fusion/Ni
0.87029

0.766521

370.1351
23970

10

DF
4
5
9

Sum of Squares

4596000.0
685000.0

$\begin{array}{lr}5 & 685000.0 \\ 9 & 5281000.0\end{array}$

Mean Square

1149000

F Ratio

Prob > F

Number

Mean
23700.0
23300.0

23300.0

24900.0

24650.0

Std Error

261.73

261.73

261.73

261.73

Number

Mean

23700.0

23300.0

24900.0
24650.0

24650.0

Std Dev

141.421
565.685

565.685

141.421
70.711

565.685
23027
22627

22627

23977

22627

Std Err Mean

$$
\begin{array}{r}
\text { Err Mean } \\
100.00 \\
400.00
\end{array}
$$

400.00
100.00

100.00
50.00

50.00
400.00
Upper 95\%

24971
28382

28382

26171

25285
28382 
WSRC-TR-2005-00166

SRNL-RPP-2005-00029

Revision 0

Exhibit A2. Statistical Comparisons of Preparation/Crucible Measurements by Element by Type of Sample

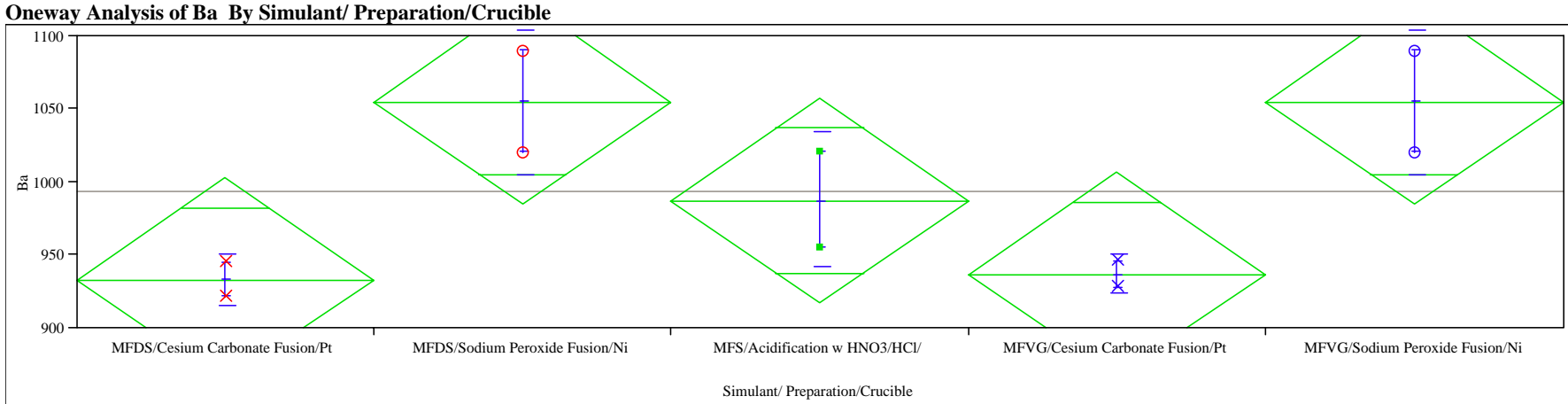

Oneway Anova

Summary of Fit

Rsquare

Adj Rsquare

Root Mean Square Error

Mean of Response

Observations (or Sum Wgts)

Analysis of Variance

Simulant/ Preparation/Crucible

Error

Means for Oneway Anova

Means

Level

MFDS/Cesium Carbonate Fusion/Pt

MFS/Aodicin Peroxide Fusion/N/

MFVG/Cicaton w HNO3/HCl/

MFVG/Sodium Peroxide Fusion/Pt

M G/Sodium Peroxide Fusion/Ni

or variance

Means and Std Deviations

Level

MFDS/Cesium Carbonate Fusion/Pt

MFDS/Sodium Peroxide Fusion/N/

MFVG/C i C W HNO3/HCI/

MFVG/Sodin Carbonate Fusion/Pt

0.794809

0.630656

38.63289

10

4
5
9

Sum of Squares

7406.000

7462.500

Number

Mean

933.00

1055.00

937.00

937.00

Mean

933.00

1055.00

987.50

937.00
7226.50
1492.50

27.318

27.318

27.318

27.318
27.318

Std Dev

16.9706

49.4975

45.9619

12.7279
49.4975

$\begin{array}{rr}\text { F Ratio } & \text { Prob > F } \\ 4.8419 & 0.0570\end{array}$

Lower 95\%

862.78
984.78

984.78
917.28

917.28
866.78

984.78

Std Err Mean

12.000

35.000

32.500

35.000
Upper 95\%

1003.2

1125.2

1057.7

1125.2

Lower $95 \%$
780.53
610.28
574.55
822.64
610.28

Upper 95\% 1085.5 1499.7

1400.5

1051.4
1499.7 
WSRC-TR-2005-00166

SRNL-RPP-2005-00029

Revision 0

Exhibit A2. Statistical Comparisons of Preparation/Crucible Measurements by Element by Type of Sample

Oneway Analysis of Ca By Simulant/ Preparation/Crucible

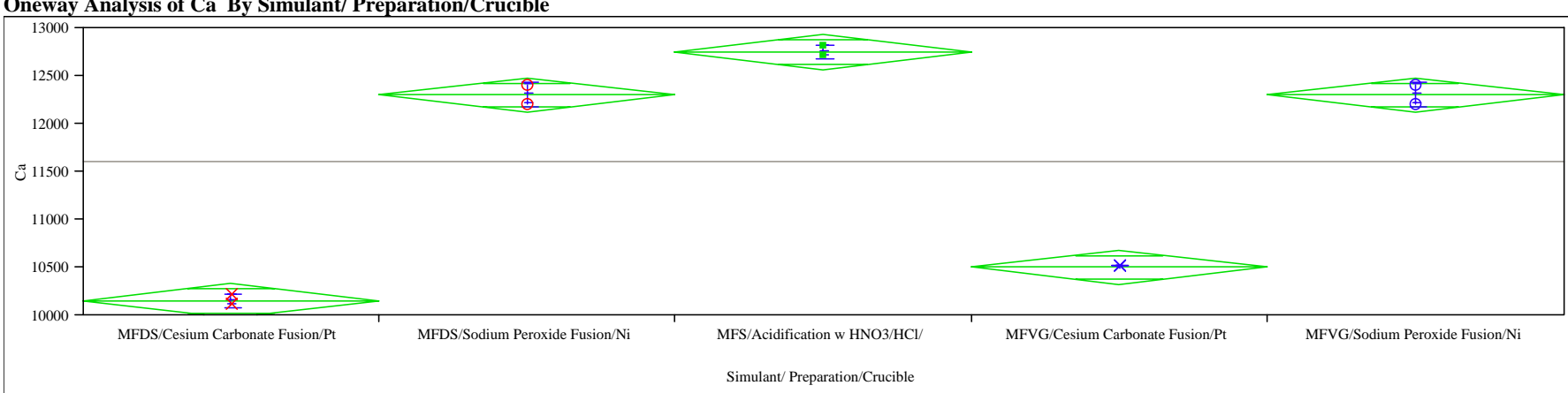

Oneway Anova

Summary of Fit

Rsquare

Adj Rsquare

Root Mean Square Erro

Mean of Response

Observations (or Sum Wgts)

Analysis of Variance

Simulant/ Preparation/Crucible

Error

Means for Oneway Anova

Level

MFDS/Sodium Peroxide Fusion/Ni

MFS/Acidification w $\mathrm{HNO} / \mathrm{HCl} /$

MFVG/Cesium Carbonate Fusion/Pt

MFVG/Sodium Peroxide Fusion/Ni

Std Error uses a pooled estimate of error variance

Means and Std Deviations

Level

MFDS/Cesium Carbonate Fusion/Pt

MFDS/Sodium Peroxide Fusion/Ni

MFS/Acidification w $\mathrm{HNO} / \mathrm{HCl} /$

MFVG/Cesium Carbonate Fusion/Pt

MFVG/Sodium Peroxide Fusion/Ni

0.995567
0.992021
100
11600
10
DF
4
5
9

100

DF
4
5
9

Sum of Squares$$
\begin{array}{r}
11230000 \\
50000
\end{array}
$$

50000
11280000

Number

$$
\begin{array}{r}
\text { Mean } \\
10150.0 \\
12300.0 \\
12750.0 \\
10500.0 \\
12300.0
\end{array}
$$

Number

Mean
10150.0
12300.0
12750.0
10500.0
12300.0
Mean Square

$$
\begin{array}{r}
2807500 \\
10000
\end{array}
$$$$
10000
$$

Std Error
70.711
70.711

70.711
70.711
70.711

70.711
70.711

70.711

Std Dev

70.711

141.421
70.711
0.000

70.711

141.421
F Ratio

280.7500

Prob > F

$\begin{array}{rr}\text { Lower 95\% } & \text { Upper 95\% } \\ 9968 & 10332 \\ 12118 & 12482 \\ 12568 & 12932 \\ 10318 & 10682 \\ 12118 & 12482 \\ & \end{array}$

Std Err Mean

50.00
100.00
50.00
0.00
100.00

100.00
50.00
0.00

50.00
100.00

Lower $95 \%$
9515
11029
12115
10500
11029

Upper 95\%

10785

13571

13385

10500
13571 
WSRC-TR-2005-00166

SRNL-RPP-2005-00029

Revision 0

Exhibit A2. Statistical Comparisons of Preparation/Crucible Measurements by Element by Type of Sample

Oneway Analysis of Cd By Simulant/ Preparation/Crucible

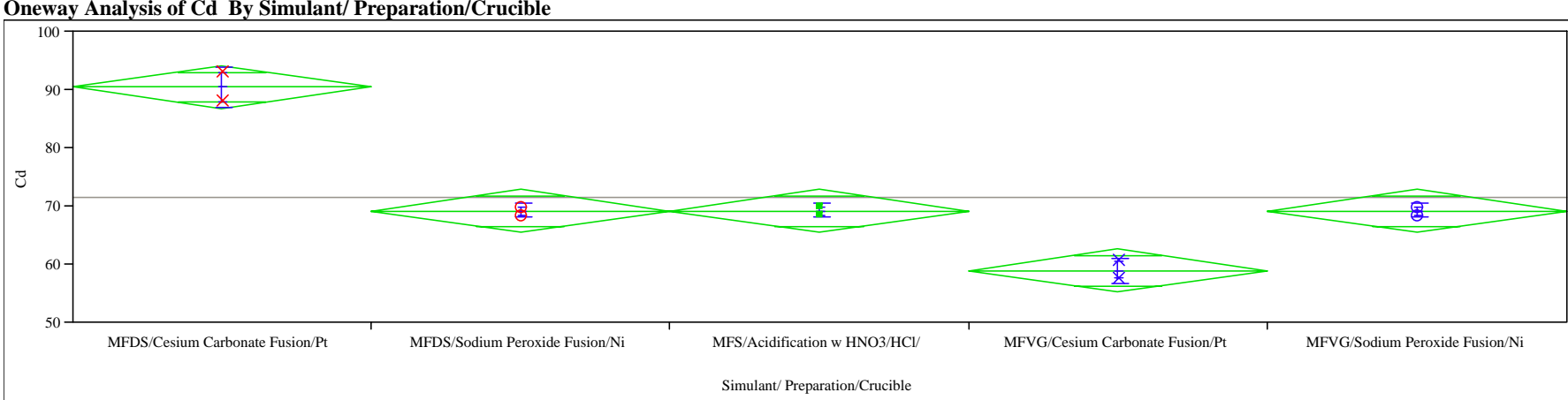

Oneway Anova

Summary of Fit

Rsquare

Adj Rsquare

Root Mean Square Error

Mean of Response

Observations (or Sum Wgts)

Analysis of Variance

Simulant/ Preparation/Crucible

Error

C. Total

Means for Oneway Anova

Level

MFDS/Cesium Carbonate Fusion/Pt

MFS/Sodium Peroxide Fusion/Ny

MFVG/Cicaton w HNOB/HCI/

MFVG/Sodium Peroxide Fusion/P.

C.Sodiun Peroxide Fusion/Ni

or variance

Means and Std Deviations

Level

MFDS/Cesium Carbonate Fusion/Pt

MFS/Sodin Peroxide Fusion/N/

MFVG/Cicaton W HNO3/HCl/

MFVG/Sodin Carbonate Fusion/Pt

0.981225

0.966205

2.018663

10

4
5
9

Sum of Squares

1064.8500

20.3750

Numbe

Mean
90.5000

90.5000
69.2500

69.2500

59.0000

69.2500

Number

Mean

90.5000

69.2500

59.0000

69.2500
266.213

$$
\begin{array}{r}
\text { F Ratio } \\
65.3282
\end{array}
$$

Prob $>$ F

Std Error

1.4274

1.4274

1.4274

1.4274

Std Dev

3.53553
1.06066

1.06066

1.06066

2.12132
1.06066

Lower 95\%
86.831
65.581
65.581
55.331
65.581


Std Err Mean
2.5000
0.7500
0.7500
1.5000
0.7500

0.0002

Upper 95\%

94.169

72.919

72.919

62.669
72.919

Lower 95\%

58.734

59.720

59.720

39.941
59.720
Upper 95\% 122.27
78.78
78.78

78.78
78.06

78.06
78.78 
WSRC-TR-2005-00166

SRNL-RPP-2005-00029

Revision 0

Exhibit A2. Statistical Comparisons of Preparation/Crucible Measurements by Element by Type of Sample

Oneway Analysis of Ce By Simulant/ Preparation/Crucible

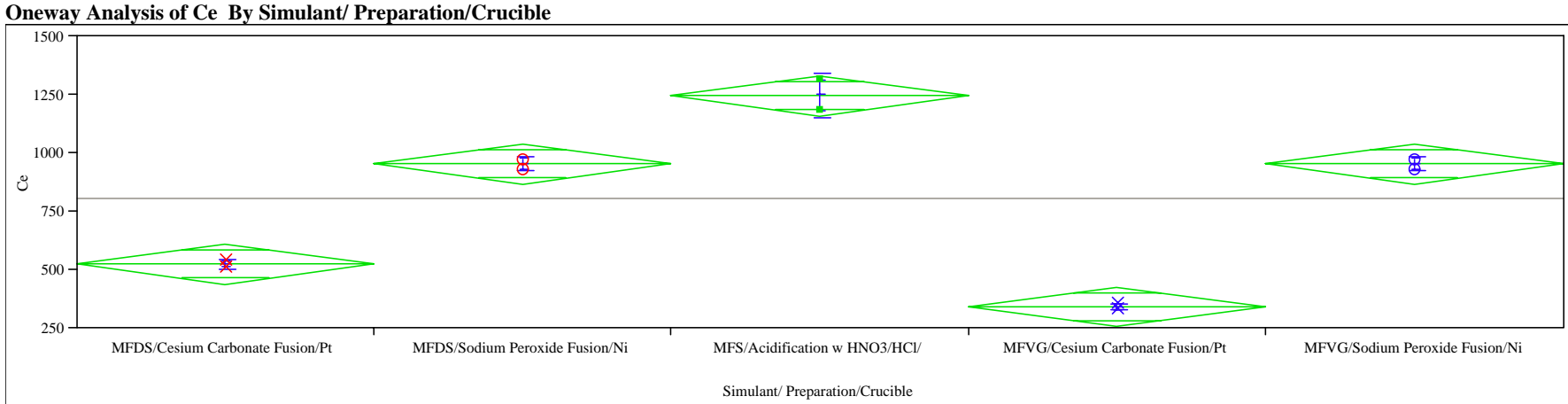

Oneway Anova

Summary of Fit

Rsquare

Adj Rsquare

Root Mean Square Error

Mean of Response

Observations (or Sum Wgts)

Analysis of Variance

Source

Simulant/ Preparation/Crucible

Error

Means for Oneway Anova

Level

MFDS/Cesium Carbonate Fusion/Pt

MFS/Aodidn Peroxide Fusion/N/

MFVG/Cicaton w HNO3/HCI/

MFVG/Sodium Peroxide Fusion/Pt

S G/Sodium Peroxide Fusion/Ni

ror variance

Means and Std Deviations

Level

MFDS/Cesium Carbonate Fusion/Pt

MFDS/Sodium Peroxide Fusion/N/

MFVG/C i C W HNOB/HCl/

MFVG/Sodin Carbonate Fusion/Pt

0.98988

0.981785

46.55132

10

4
5
9

Sum of Squares

059872.9

$\begin{array}{rr}5 & 10835.1 \\ 9 & 1070708.0\end{array}$

Mean Square

264968

F Ratio

Prob $>$ F

Number

Mean Std Error

Std Error
32.917

32.917
32.917

953.00

1245.00

342.25

32.917

32.917

440.4
868.4

868.4
1160.4

257.6
868.4

Std Dev

Number

Mean
525.00

525.00

953.00

(245.00

342.25

21.2132

29.6985

91.9239

13.0815

Std Err Mean

15.000

21.000

6.000

21.000

$$
<.0001
$$

Upper 95\%

609.6

1037.6

1329.6
426.9

426.9
1037.6

Lower 95\%
334.41
686.17
419.10
224.72
686.17

Upper 95\%

715.6

1219.8
2070.9

459.8 
WSRC-TR-2005-00166

SRNL-RPP-2005-00029

Revision 0

Exhibit A2. Statistical Comparisons of Preparation/Crucible Measurements by Element by Type of Sample

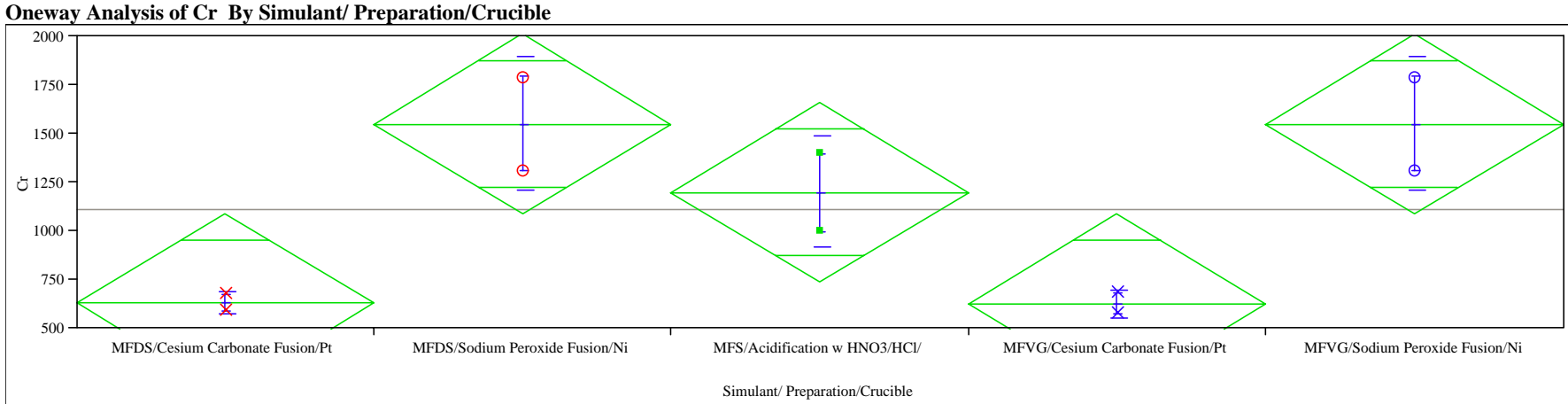

Oneway Anova

Summary of Fit

Rsquare

Adj Rsquare

Root Mean Square Erro

Mean of Response

Observations (or Sum Wgts)

Analysis of Variance

Simulant/ Preparation/Crucible

Error

Means for Oneway Anova

Means

MFDS/Cesium Carbonate Fusion/Pt

MFDS/Sodium Peroxide Fusion/ $\mathrm{N}$

MFVG/Cesiuton w HNO3/HCI/

MFVG/Sodium Peronide Fusion/Pt

ST/Sodium Peroxide Fusion/Ni

ror variance

Means and Std Deviations

Level

MFDS/Cesium Carbonate Fusion/Pt

MFS/Sodium Peroxide Fusion/N/

MFVG/Cinition w HNO3/HCl/

MFVG/Sodin Carbonate Fusion/Pt

0.84289

0.717202

252.4999

10.2
10

4
5
9

Sum of Squares

1710248.6

318781.0
2029029.6

Numbe

Mean

632.50

1550.00

1200.00
628.50

1550.00

Number

Mean
632.50
1550.00
1200.00
628.50
1550.00

Mean

632.50
1550.00

1550.00
Mean Square
42756
td Error
178.54
178.54
178.54
178.5
Std Dev
57.276
339.411
282.843
71.418
339.411

F Ratio

Prob > F

Lower 95\%
173.5
1091.0
741.0
169.5
1091.0

Std Err Mean
40.50
240.00
200.00
50.50
240.00

Upper 95\%

1091.5

2009.0

1659.0

1087.5
Lower 95\%
118
-1499
-1341
-13
-1499


WSRC-TR-2005-00166

SRNL-RPP-2005-00029

Revision 0

Exhibit A2. Statistical Comparisons of Preparation/Crucible Measurements by Element by Type of Sample

Oneway Analysis of $\mathrm{Cu}$ By Simulant/Preparation/Crucible

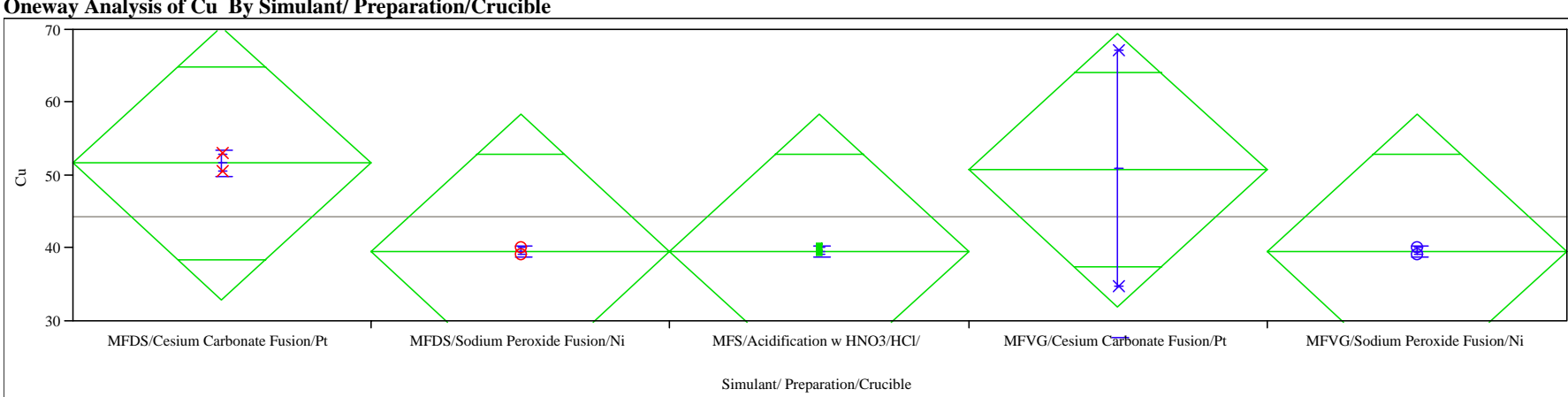

Oneway Anova

Summary of Fit

Rsquare

Adj Rsquare

Root Mean Square Error

Mean of Response

Observations (or Sum Wgts)

Analysis of Variance

Simulant/ Preparation/Crucible

Error

Means for Oneway Anova

Means

MFDS/Cesium Carbonate Fusion/Pt

MFS/Aodicim Peroxide Fusion/T/

MFVG/Cesiuto W HNOB/HCI/

MFVG/Sodium Peroxide Fusion/Pt

Sterions

ror variance

Means and Std Deviations

Level

MFDS/Cesium Carbonate Fusion/Pt

MFS/Sodin Peroxide Fusion/Ny

MFVG/C i C W HNOB/HCl/

MFVG/Sodin Carbonate Fusion/Pt

0.380014

$-0.11597$

44.31

Sum of Squares

326.54400

532.75000

Numbe

$$
\begin{array}{r}
\text { Mean } \\
51.7500
\end{array}
$$

51.7500

39.6500

50.8500

50.8500

(1)

Number

Mean
51.7500
39.6500
39.6500
50.8500
39.6500
81.63

$$
106.550
$$

Std Error

7.2990

7.2990

7.2990

7.2990

Std Dev

1.7678

0.7071

0.7071

0.7071
F Ratio

0.7662

Prob $>$ F

0.5902

$\begin{array}{rr}\text { Lower 95\% } & \text { Upper } 95 \% \\ 32.987 & 70.513 \\ 20.887 & 58.413 \\ 20.887 & 58.413 \\ 32.087 & 69.613 \\ 20.887 & 58.413\end{array}$

Std Err Mean

1.250
0.500

0.500

0.500
16.250

0.500

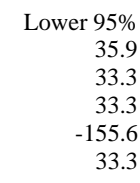

Upper 95\%

67.63

46.00

257.33
46.00 
WSRC-TR-2005-00166

SRNL-RPP-2005-00029

Revision 0

Exhibit A2. Statistical Comparisons of Preparation/Crucible Measurements by Element by Type of Sample

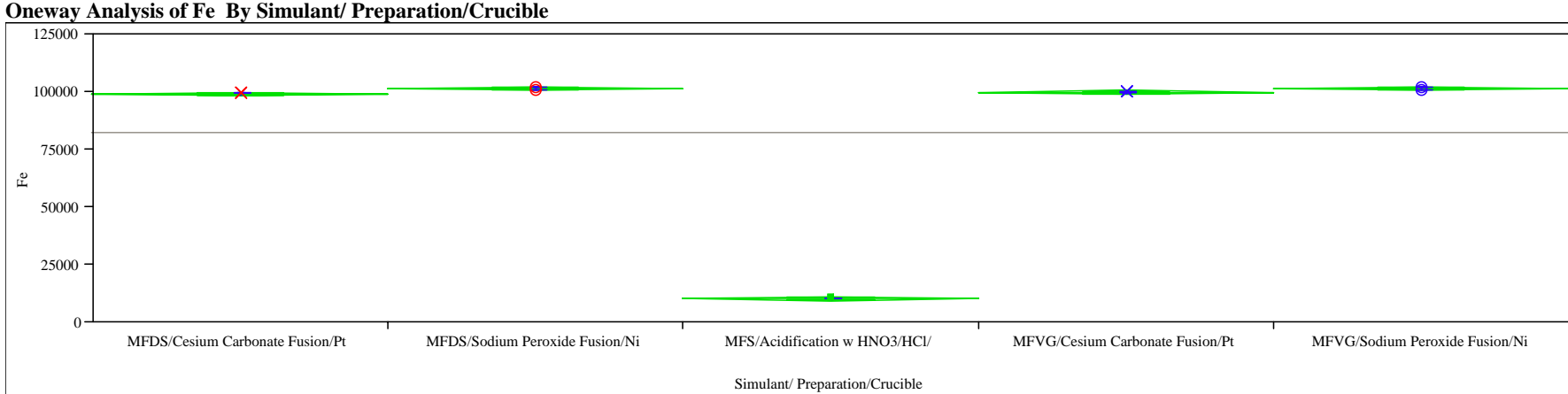

Oneway Anova

Summary of Fit

Rsquare

Adj Rsquare

Root Mean Square Erro

Mean of Response

Observations (or Sum Wgts)

Analysis of Variance

Simulant/Preparation/Crucible

C. Total

Means for Oneway Anova

Level

MFDS/Cesium Carbonate Fusion/Pt

MFDS/Sodium Peroxide Fusion/Ni

MFS/Acidification w $\mathrm{HNO} / \mathrm{HCl}$

MFVG/Cesium Carbonate Fusion/Pt

Std Error uses a pooled estimate of error variance

Means and Std Deviations

Level

MFDS/Cesium Carbonate Fusion/Pt

MFDS/Sodium Peroxide Fusion/Ni

MFS/Acidification w $\mathrm{HNO} / \mathrm{HCl} /$

MFVG/Cesium Carbonate Fusion/Pt

MFVG/Sodium Peroxide Fusion/Ni

0.999921
0.999858
452.7693
82470
10
DF
4
5
9

DF
4
5
9

Sum of Squares $1.30298 \mathrm{e} 10$

$1.30308 \mathrm{e} 10$

$\begin{array}{rr}\text { Number } & \text { Mean } \\ 2 & 99100 \\ 2 & 101500 \\ 2 & 10300 \\ 2 & 99950 \\ 2 & 101500 \\ & \\ & \\ \text { Number } & \text { Mean } \\ 2 & 99100 \\ 2 & 101500 \\ 2 & 10300 \\ 2 & 99950 \\ 2 & 101500\end{array}$

Mean Square

$3.25745 \mathrm{e} 9$

F Ratio

Prob $>$ F

205000

Std Error

320.16

320.16

320.16

320.16

Std Dev

141.421
707.107

0.000

0.000
70.711

707.107

$\begin{array}{rr} & \\ 98277 & \text { Upper } 95 \% \\ 100677 & 99923 \\ 9477 & 102323 \\ 99127 & 11123 \\ 100677 & 100773 \\ & 102323\end{array}$

Std Err Mean

100.00
500.00
0.00

500.00
0.00
50.00

50.00
Upper 95\%

100371
107853

10300

10300
100585

100585
107853 
WSRC-TR-2005-00166

SRNL-RPP-2005-00029

Revision 0

Exhibit A2. Statistical Comparisons of Preparation/Crucible Measurements by Element by Type of Sample

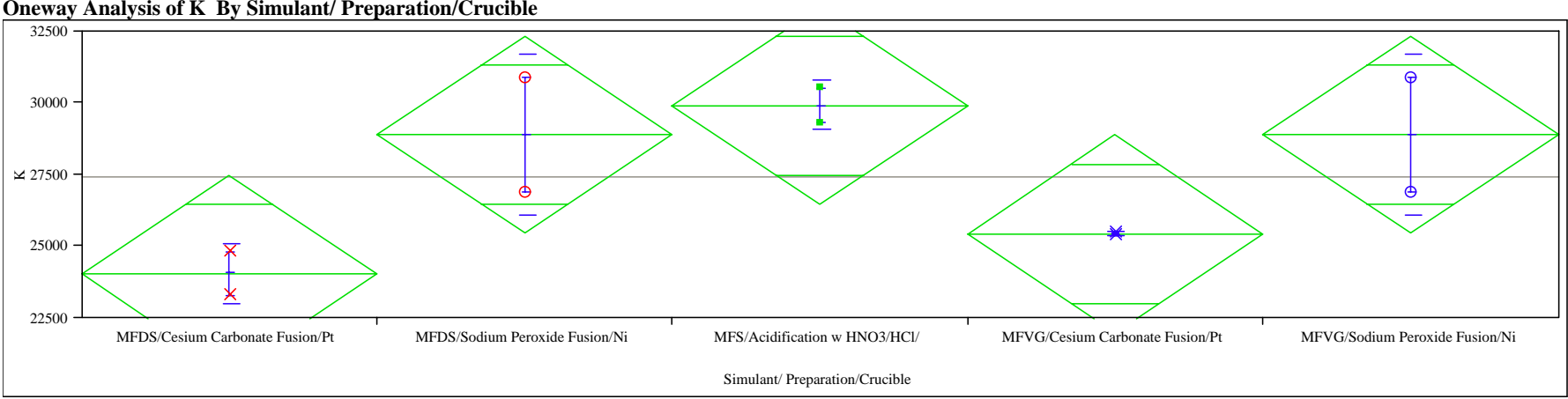

Oneway Anova

Summary of Fit

Rsquare

Adj Rsquare

Root Mean Square Erro

Mean of Response

Observations (or Sum Wgts)

Analysis of Variance

Simulant/ Preparation/Crucible

Error

Means for Oneway Anova

Level

MFDS/Sodium Peroxide Fusion/Ni

MFS/Acidification w $\mathrm{HNO} / \mathrm{HCl} /$

MFVG/Cesium Carbonate Fusion/Pt

MFVG/Sodium Peroxide Fusion/Ni

Std Error uses a pooled estimate of error variance

Means and Std Deviations

Level

MFDS/Cesium Carbonate Fusion/Pt

MFDS/Sodium Peroxide Fusion/Ni

MFS/Acidification w $\mathrm{HNO} / \mathrm{HCl}$

MFVG/Cesium Carbonate Fusion/Pt

MFVG/Sodium Peroxide Fusion/Ni

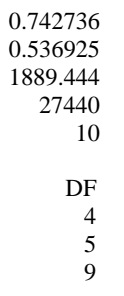

0.742736

0.536925

1889.444

27440
10

DF
4
5
9

Sum of Squares

51534000

17850000

69384000

Numbe

$$
\begin{array}{r}
\text { Mean } \\
24050.0 \\
28900.0 \\
29900.0 \\
25450.0 \\
28900.0
\end{array}
$$

$\begin{array}{rr}\text { Number } & \text { Mean } \\ 2 & 24050.0 \\ 2 & 28900.0 \\ 2 & 29900.0 \\ 2 & 25450.0 \\ 2 & 28900.0\end{array}$

Mean
24050.0
28900.0
29900.0
25450.0
28900.0
Mean Square

12883500
3570000

3.6088

Prob > F

Std Error

1336.0
1336.0

1336.0

1336.0

1336.0

Std Dev

1060.66
2828.43

2828.43
848.53

848.53
70.71

2828.43

$\begin{array}{rr}\text { Lower 95\% } & \text { Upper 95\% } \\ 20616 & 27484 \\ 25466 & 32334 \\ 26466 & 33334 \\ 22016 & 28884 \\ 25466 & 32334\end{array}$

Std Err Mean

750.0
2000.0
600.0
50.0
2000.0

Lower 95\%

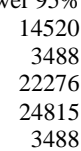

Upper 95\%

33580
54312

54312
37524

57524
26085

54312 
WSRC-TR-2005-00166

SRNL-RPP-2005-00029

Revision 0

Exhibit A2. Statistical Comparisons of Preparation/Crucible Measurements by Element by Type of Sample

Oneway Analysis of La By Simulant/ Preparation/Crucible

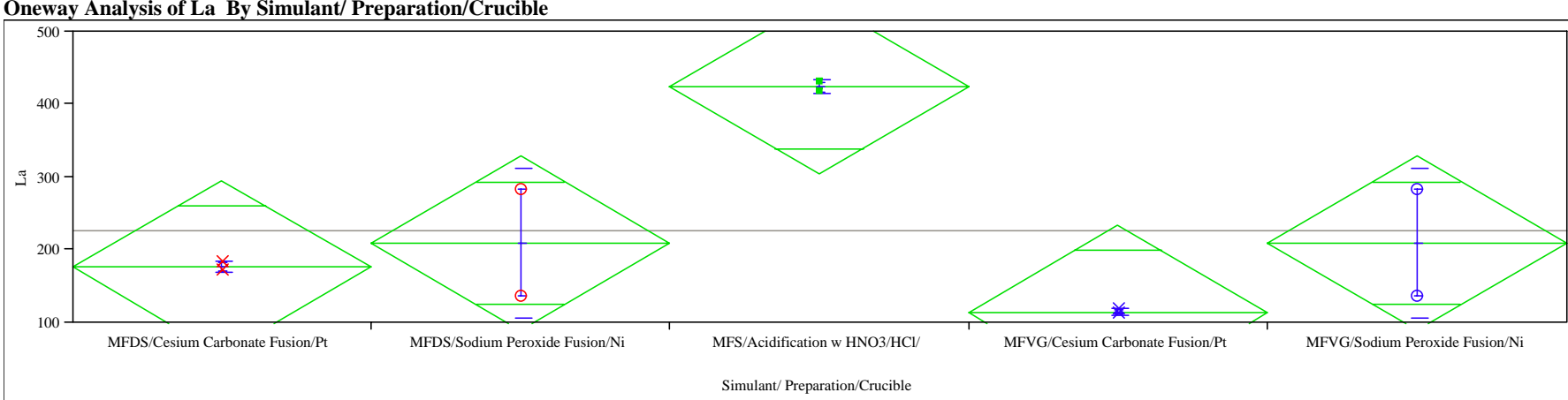

Oneway Anova

Summary of Fit

Rsquare

Adj Rsquare

Root Mean Square Error

Mean of Response

Observations (or Sum Wgts)

Analysis of Variance

Source

Simulant/ Preparation/Crucible

Error

C. Total

Means for Oneway Anova

Level

MFDS/Cesium Carbonate Fusion/Pt

MFS/Aodin Peroxide Fusion/Ni

MFVG/Cicaton w HNOB/HCI/

MFVG/Sodium Peroxide Fusion/Pt

S G/Sodiun Peroxide Fusion/Ni

or variance

Means and Std Deviations

MFDel

MFDS/Cesium Carbonate Fusion/Pt

MTS/Sodin Peroxide Fusion/Ny

MFVG/Cicaton w HNOB/HCl/

MFVG/Sodin Carbonate Fusion/Pt

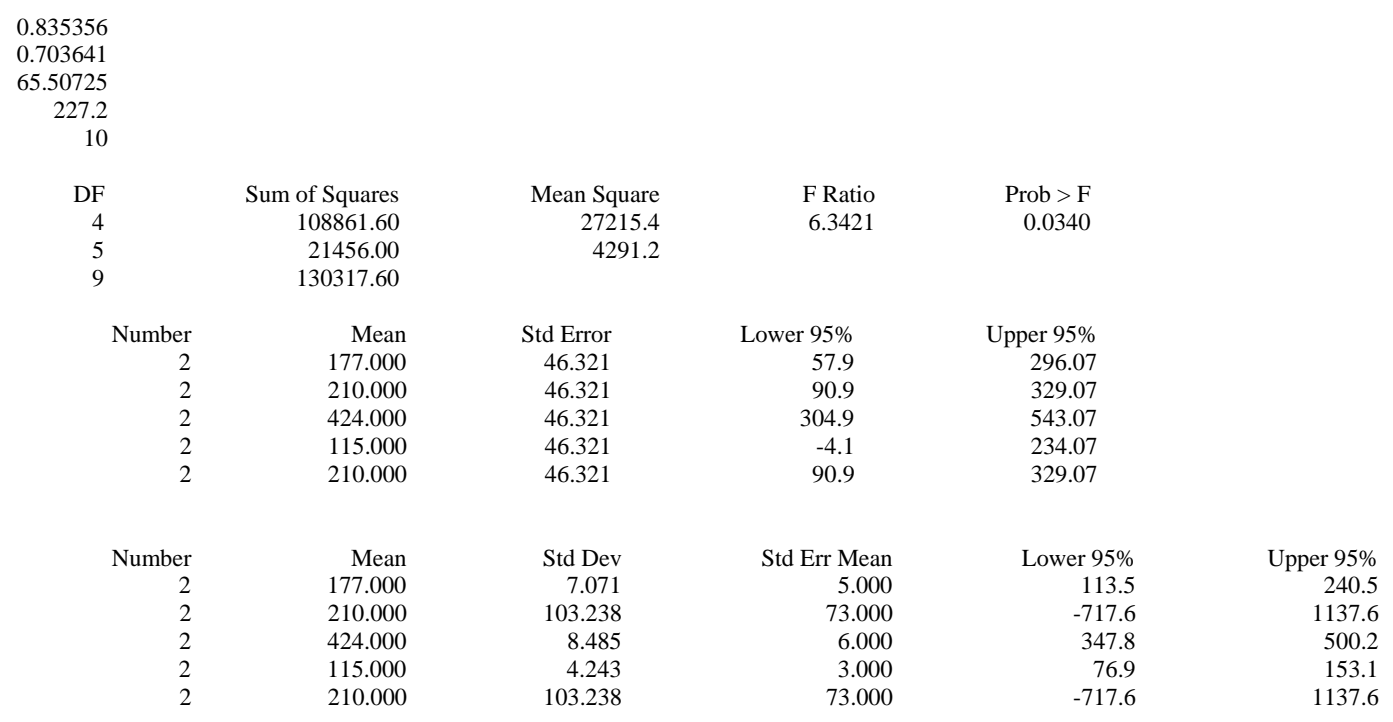


WSRC-TR-2005-00166

SRNL-RPP-2005-00029

Revision 0

Exhibit A2. Statistical Comparisons of Preparation/Crucible Measurements by Element by Type of Sample

Oneway Analysis of Li By Simulant/Preparation/Crucible

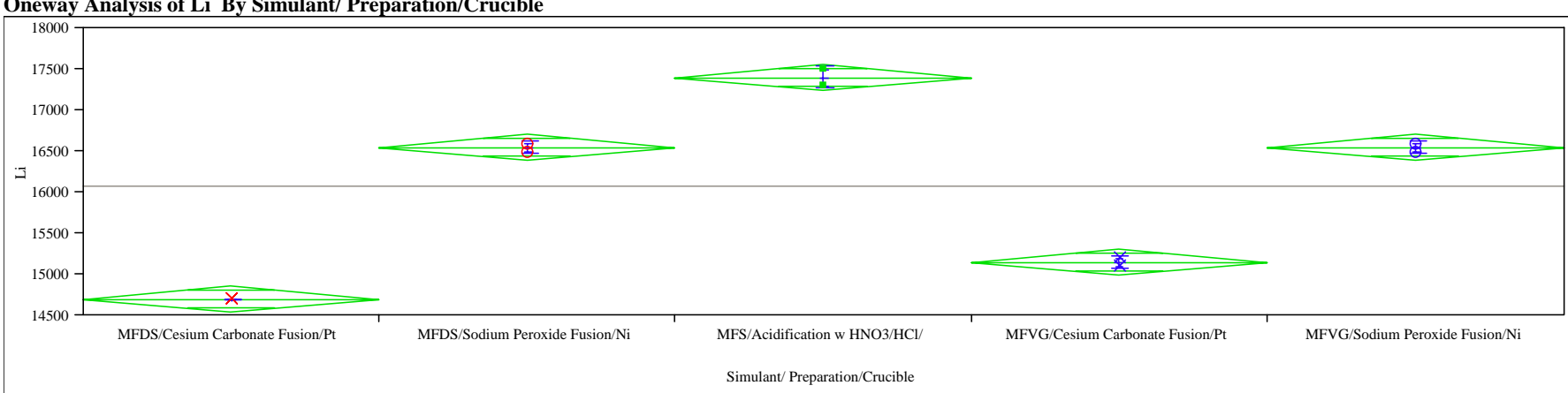

Oneway Anova

Summary of Fit

Rsquare

Adj Rsquare

Root Mean Square Erro

Mean of Response

Observations (or Sum Wgts)

Analysis of Variance

Simulant/ Preparation/Crucible

Error

Means for Oneway Anova

Level

MFDS/Sodium Peroxide Fusion/Ni

MFS/Acidification w $\mathrm{HNO} / \mathrm{HCl} /$

MFVG/Cesium Carbonate Fusion/Pt

MFVG/Sodium Peroxide Fusion/Ni

Std Error uses a pooled estimate of error variance

Means and Std Deviations

Level

MFDS/Cesium Carbonate Fusion/Pt

MFDS/Sodium Peroxide Fusion/Ni

MFS/Acidification w HNO3/HCl/

MFVG/Cesium Carbonate Fusion/Pt

MFVG/Sodium Peroxide Fusion/Ni

0.996479
0.993663
83.666
16070
10
DF
4
5
9

4
5
9

Sum of Squares

9906000.0

9941000.0

$\begin{array}{rr}\text { Number } & \text { Mean } \\ 2 & 14700.0 \\ 2 & 16550.0 \\ 2 & 17400.0 \\ 2 & 15150.0 \\ 2 & 16550.0 \\ & \\ \text { Number } & \text { Mean } \\ 2 & 14700.0 \\ 2 & 16550.0 \\ 2 & 17400.0 \\ 2 & 15150.0 \\ 2 & 16550.0\end{array}$

$$
\begin{array}{r}
\text { Mean Square } \\
2476500 \\
7000 \\
\\
\\
\text { Std Error } \\
59.161 \\
59.161 \\
59.161 \\
59.161 \\
59.161 \\
\\
\\
\text { Std Dev } \\
0.000 \\
70.711 \\
141.421 \\
70.711 \\
70.711
\end{array}
$$$$
2476500
$$$$
\begin{array}{r}
2476500 \\
7000
\end{array}
$$

Prob $>$ F

353.7857

$<.0001$

$\begin{array}{rr}\text { Lower } 95 \% & \text { Upper } 95 \% \\ 14548 & 14852 \\ 16398 & 16702 \\ 17248 & 17552 \\ 14998 & 15302 \\ 16398 & 16702 \\ & \end{array}$

Std Err Mean

0.00
50.00
100.00

100.00

50.00
50.00
Upper 95\%

14700
17185

17185
18671

18671
15785

15785
17185 
WSRC-TR-2005-00166

SRNL-RPP-2005-00029

Revision 0

Exhibit A2. Statistical Comparisons of Preparation/Crucible Measurements by Element by Type of Sample

Oneway Analysis of Mg By Simulant/Preparation/Crucible

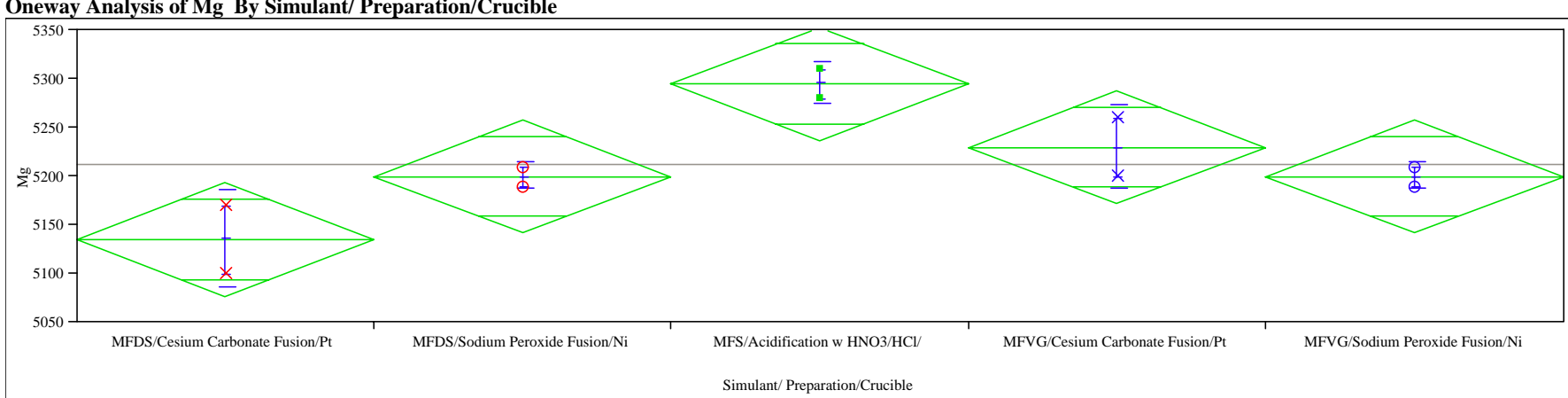

Oneway Anova

Summary of Fit

Rsquare

Adj Rsquare

Root Mean Square Erro

Mean of Response

Observations (or Sum Wgts)

Analysis of Variance

Simulant/ Preparation/Crucible

Error

C. Total

Means for Oneway Anova

Level

MFDS/Cesium Carbonate Fusion/Pt

MFS/Sodium Peroxide Fusion/N/

MFVG/Cesiut Can wNOB/

MFVG/Sodium Peroxide Fusion/Pt

MFV G/Sodium Peroxide Fusion/Ni

ror variance

Means and Std Deviations

Level

MFDS/Cesium Carbonate Fusion/Pt

MFDS/Sodium Peroxide Fusion/N

MFVG/Cicaton w HNO3/HCl/

MFVG/Sodin Carbonate Fusion/Pt

0.840426

0.712766

51.93744

5212
10
DF
4
5
9

Sum of Squares

5100.000

5100.000

Numbe

$$
\begin{array}{r}
\text { Mean } \\
5135.00 \\
5200.00 \\
5295.00 \\
5230.00 \\
5200.00
\end{array}
$$

Number

Mean
5135.00
5200.00
5295.00
5230.00
5200.00

Mean Square 6715.00 1020.00

Std Error

$$
\begin{aligned}
& 22.583 \\
& 22.583
\end{aligned}
$$$$
22.583
$$$$
22.583
$$$$
-5
$$

Std Dev

49.4975

14.1421

21.2132

14.1421
F Ratio

6.5833 Prob > F

Lower 95\% Upper 95\%

$5076.9-5193.1$

$\begin{array}{ll}5141.9 & 5258.1 \\ 5236.9 & 5353.1\end{array}$

$\begin{array}{ll}5236.9 & 5353.1 \\ 5171.9 & 5288.1\end{array}$

$\begin{array}{lr}517.9 & 5353.1 \\ 5141.9- & 5258.1\end{array}$

Std Err Mean

35.000

10.000

15.000

10.000

Lower $95 \%$
4690.3
5072.9
5104.4
4848.8
5072.9

Upper 95\%

5579.7

5327.1

5485.6

5327.1 
WSRC-TR-2005-00166

SRNL-RPP-2005-00029

Revision 0

Exhibit A2. Statistical Comparisons of Preparation/Crucible Measurements by Element by Type of Sample

Oneway Analysis of Mn By Simulant/Preparation/Crucible

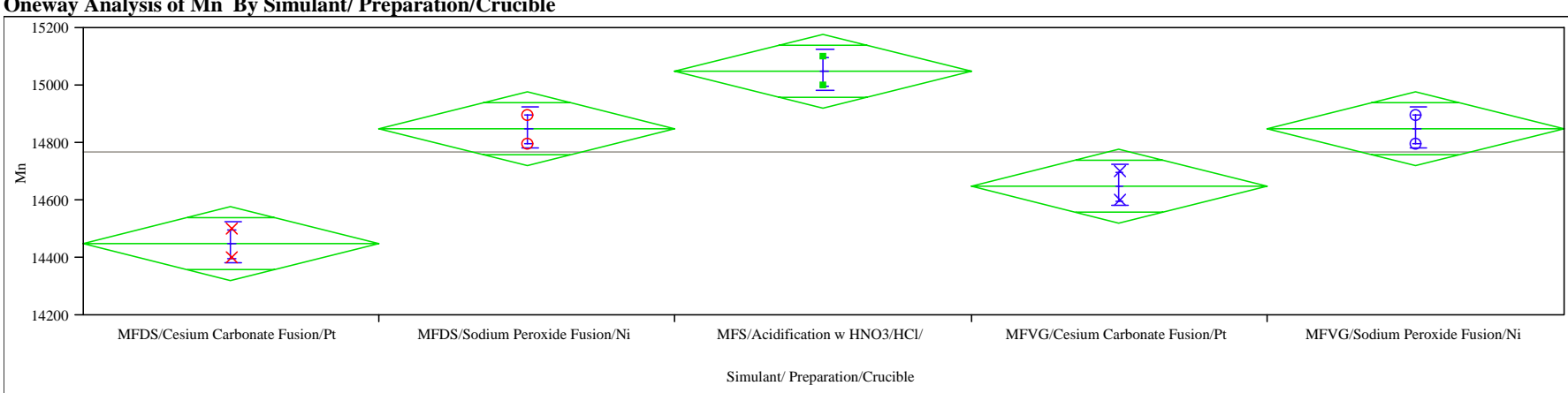

Oneway Anova

Summary of Fit

Rsquare

Adj Rsquare

Root Mean Square Erro

Mean of Response

Observations (or Sum Wgts)

Analysis of Variance

Simulant/Preparation/Crucible

C. Total

Means for Oneway Anova

Level

MFDS/Cesium Carbonate Fusion/Pt

MFDS/Sodium Peroxide Fusion/Ni

MFS/Acidification w $\mathrm{HNO} / \mathrm{HCl} /$

MFVG/Cesium Carbonate Fusion/Pt

Std Error uses a pooled estimate of error variance

Means and Std Deviations

Level

MFDS/Cesium Carbonate Fusion/Pt

MFDS/Sodium Peroxide Fusion/Ni

MFS/Acidification w HNO3/HCl/

MFVG/Cesium Carbonate Fusion/Pt

MFVG/Sodium Peroxide Fusion/Ni

0.943311
0.897959
70.71068
14770
10
DF
4
5
9

.943311

10

F
5
5
9

Sum of Squares

416000.00

25000.00
441000.00

Number Mean

Mean
14450.0

14850.0

15050.0
14650.0

14850.0

Number

Mean
14450.0
14850.0
15050.0
14650.0
14850.0
Mean Square

$$
\begin{array}{r}
104000 \\
5000
\end{array}
$$

F Ratio

20.8000

Prob $>$ F

Std Error
50.000

50.000
50.000
50.000

50.000

50.000

Std Dev
70.7107

70.7107
70.7107

70.7107
70.7107

70.7107
70.7107

70.7107
Lower 95\%

14321
14721
14921

14921
14521
14721

Std Err Mean

50.000
50.000
50.000

50.000

50.000
50.000
Upper 95\%

14579
14979

14979
15179

15179
14779

14779
14979

Lower 95\%
13815

13815
14215
14415

14215
14415
14015

14015
14215
Upper 95\%

15085
15485

15485
15685

15285

15285
15485 
WSRC-TR-2005-00166

SRNL-RPP-2005-00029

Revision 0

Exhibit A2. Statistical Comparisons of Preparation/Crucible Measurements by Element by Type of Sample

Oneway Analysis of Mo By Simulant/ Preparation/Crucible

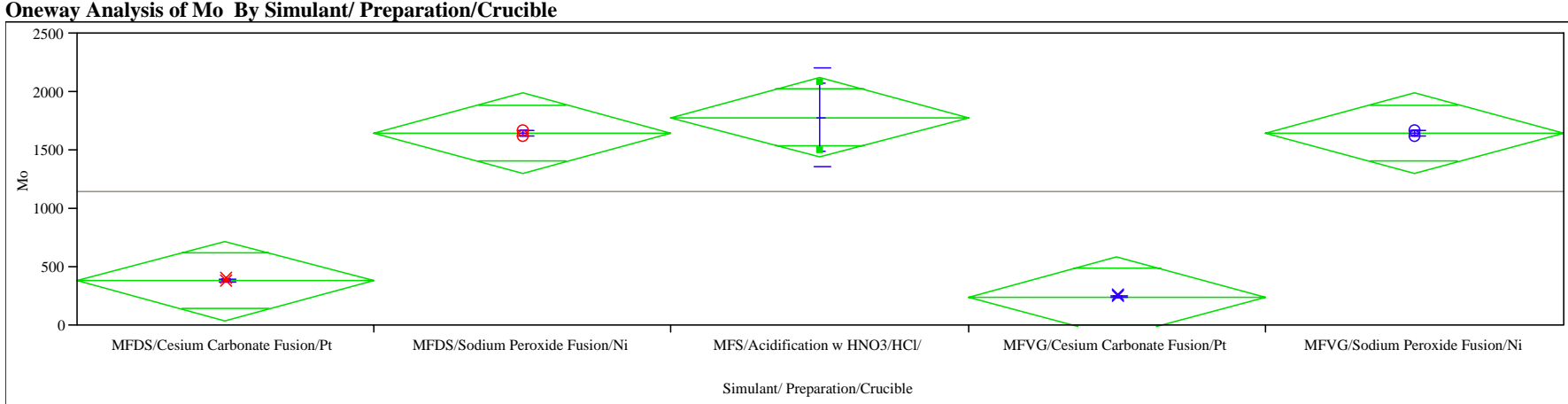

Oneway Anova

Summary of Fit

Rsquare

Adj Rsquare

Root Mean Square Error

Mean of Response

Observations (or Sum Wgts)

Analysis of Variance

Simulant/ Preparation/Crucible

Error

Means for Oneway Anova

Level

MFDS/Cesium Carbonate Fusion/Pt

MFS/Aodidin Pexoxide Fusion//T/

MFVG/Cesiuton W HNO3/HCl/

MFVG/Sodium Peroxide Fusion/Pt

S G/Sodium Peroxide Fusion/Ni

ror variance

Means and Std Deviations

Level

MFDS/Cesium Carbonate Fusion/Pt

MFS/Sodin Peroxide Fusion/Ny

MFVG/Cin w

MFVG/Sodium Peroxide Fusion/Ni

0.963132

0.933638

187.605

10

DF

4
5
9

Sum of Squares 4597299.1

175979.1

Numbe

Mean

384.75

1650.00

1785.00
250.00

1650.00

Number

Mean
384.75
1650.00
1785.00
250.00
1650.00

Mean
384.75

1650.00

1785.00

1650.00
1149325

35196

Std Error

132.66

132.66

132.66

132.66

Std Dev

15.203
28.284

417.193

9.899
28.284
F Ratio

32.6551

Prob $>$ F

Lower 95\% _ _ Upper 95\%

pper $95 \%$
725.8

725.8
1991.0

2126.0

591.0

$\begin{array}{rr}1444 & 2126.0 \\ -91 & 591.0 \\ 1309 & 1991.0\end{array}$

Std Err Mean

10.75
20.00
295.00
7.00

Lower 95\%

248
1396
-1963
161

1396
Upper 95\%

521.3

1904.1
5533.3

338.9 
WSRC-TR-2005-00166

SRNL-RPP-2005-00029

Revision 0

Exhibit A2. Statistical Comparisons of Preparation/Crucible Measurements by Element by Type of Sample

Oneway Analysis of Na By Simulant/Preparation/Crucible

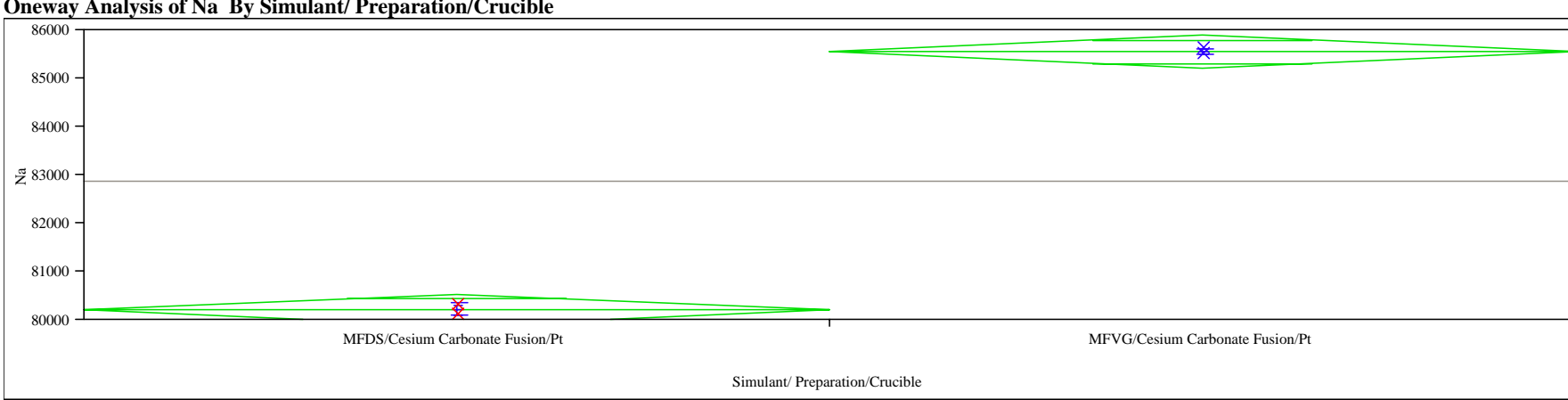

Missing Rows

6Oneway Anova

Summary of Fit

Rsquare

Adj Rsquare

Root Mean Square Error

Mean of Response

Observations (or Sum Wgts)

MFDS/Cesium Carbonate Fusion/Pt-MFVG/Cesium Carbonate Fusion/Pt

Assuming equal variances

Difference

Std Err Dif

Upper CL Dif

Lower CL Dif

-5350.0 t Ratio

$111.8 \mathrm{DF}$
$-4868.9 \mathrm{Prob}>\mathrm{t}$

-4868.9 Prob $>|t|$

0.95 Prob $<\mathrm{t}$

$-47.8519$

2
0.0004

0.9998

0.0002

Analysis of Variance

Analysis
Source
Simulat

Simulant/ Preparation/Crucible

Error

Means for Oneway Anova

Level

MFDS/Cesium Carbonate Fusion/Pt

MFVG/Cesium Carbonate Fusion/Pt

Std Error uses a pooled estimate of error variance

Means and Std Deviations

Level

MFDS/Cesium Carbonate Fusion/Pt

MFVG/Cesium Carbonate Fusion/Pt

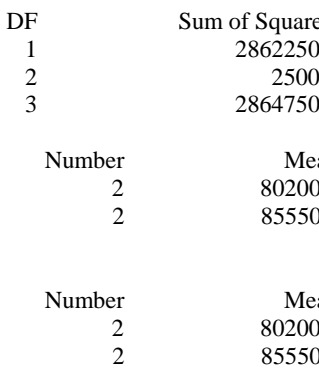

$\begin{array}{rrr}\text { Mean Square } & \text { F Ratio } & \text { Prob }>\text { F } \\ 28622500 & 2289.8 & 0.0004 \\ 12500 & & \\ & & \\ \text { Std Error } & \text { Lower 95\% } & \text { Upper 95\% } \\ 79.057 & 79860 & 80540 \\ 79.057 & 85210 & 85890 \\ & & \\ \text { Std Dev } & \text { Std Err Mean } & \text { Lower 95\% } \\ 141.421 & 100.00 & 78929 \\ 70.711 & 50.00 & 84915\end{array}$

Upper 95\%

$\begin{array}{ll}78929 & 81471 \\ 84915 & 86185\end{array}$


WSRC-TR-2005-00166

SRNL-RPP-2005-00029

Revision 0

Exhibit A2. Statistical Comparisons of Preparation/Crucible Measurements by Element by Type of Sample

Oneway Analysis of Ni By Simulant/Preparation/Crucible

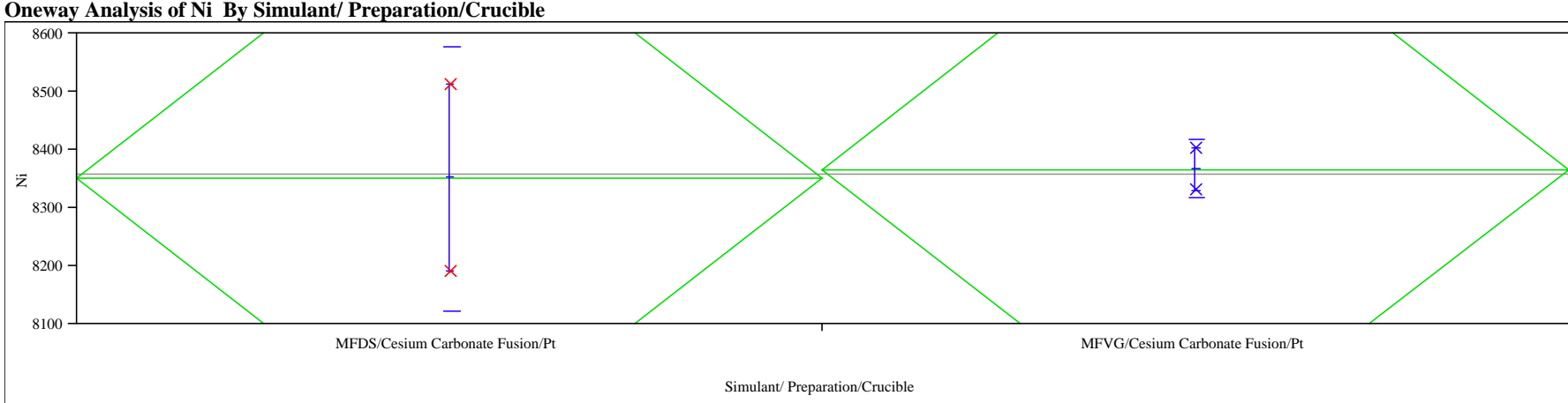

Missing Rows

eway Anova

Summary of Fit

Rsquare

Adj Rsquare

Root Mean Square Error

Mean of Respons

Observations (or Sum Wgts)

nnt/ Preparation/Crucible

MFDS/Cesium Ca bonate Fusion/Pt-MFVG/Cesinn Ca bonat FusontPt

Assuming equal variances

Difference

Std Err Dif

Upper CL Dif

Lower CL Dif

-15.00 t Ratio

163.78 DF

$\begin{array}{rl}-719.70 \text { Prob }>t & t\end{array}$

-0.09158
2
0.9354

0.95 Prob $<\mathrm{t}$

0.9354

0.5323

Analysis of Variance

Source

Simulant/ Preparation/Crucible

Error

C. Total

Means for Oneway Anova

Level

MFDS/Cesium Carbonate Fusion/Pt

(1)

error variance

(a)

MFDS/Cesium Carbonate Fusion/Pt

MFVG/Cesium Carbonate Fusion/Pt

\begin{tabular}{|c|c|c|c|c|c|}
\hline DF & Sum of Squares & Mean Square & F Ratio & Prob $>F$ & \\
\hline 1 & 225.000 & 225.0 & 0.0084 & 0.9354 & \\
\hline 2 & 53650.000 & 26825.0 & & & \\
\hline 3 & 53875.000 & & & & \\
\hline Number & Mean & Std Error & Lower 95\% & Upper 95\% & \\
\hline 2 & 8350.00 & 115.81 & 7851.7 & 8848.3 & \\
\hline 2 & 8365.00 & 115.81 & 7866.7 & 8863.3 & \\
\hline Number & Mean & Std Dev & Std Err Mean & Lower 95\% & Upper 95\% \\
\hline 2 & 8350.00 & 226.274 & 160.00 & 6317.0 & 10383 \\
\hline 2 & 8365.00 & 49.497 & 35.00 & 7920.3 & 8810 \\
\hline
\end{tabular}


WSRC-TR-2005-00166

SRNL-RPP-2005-00029

Revision 0

Exhibit A2. Statistical Comparisons of Preparation/Crucible Measurements by Element by Type of Sample

Oneway Analysis of P By Simulant/Preparation/Crucible

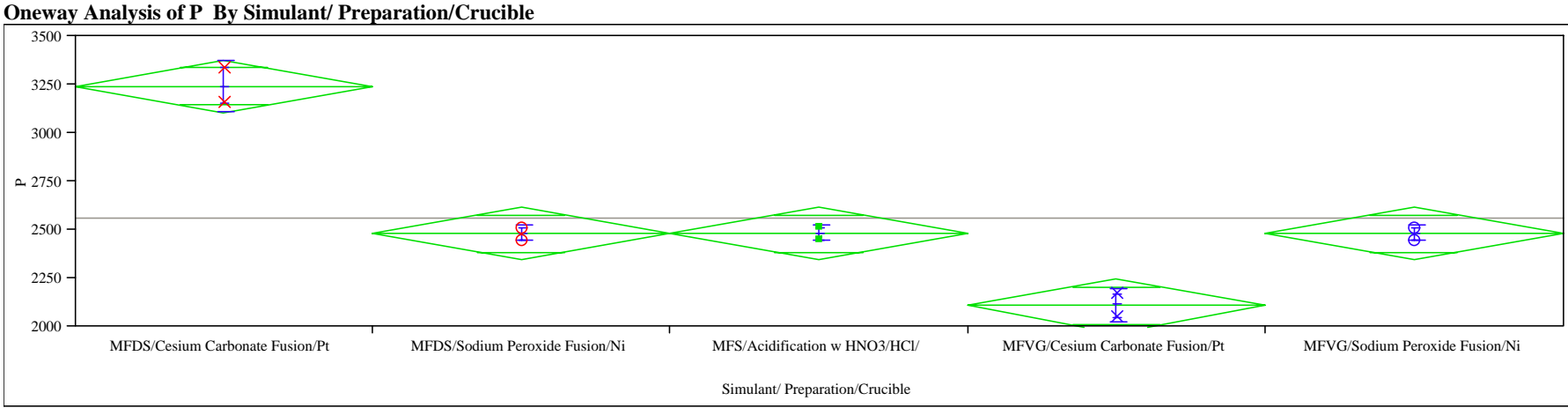

Oneway Anova

Summary of Fit

Rsquare

Adj Rsquare

Mean of Response

Observations (or Sum Wgts)

Analysis of Variance

Source

Simulant/ Preparation/Crucible

Error

C. Total

Mens for Oneway Anova

Level

MFDS/Cesium Carbonate Fusion/Pt

MFS/Sodum Peroxide Fusion/Ni

MFVG/Cinication w HNO3/HCI/

MFVG/Sodium Paroxion/Pt

S G/Sodium Peroxide Fusion/Ni

ror variance

Means and Std Deviations

Level

MFDS/Cesium Carbonate Fusion/Pt

MDS/Sodin Peroxide Fusion/N

MFVG/Cicaton w HNO3/HCl/

MFVG/Sodin Carbonate Fusion/Pt

\subsection{1}

0.963749

75.11658
2557.5

10

DF
4
5
9

Sum of Squares

372650.0

28212.5

Number

Mean

3240.00

2480.00

2480.00

2107.50

(1)

Number

Mean
3240.00
2480.00
2480.00
2107.50
2480.00
343163

Std Error

53.115

53.115

53.115

53.115
53.115

Std Dev

127.279

42.426

42.426

81.317
42.426
F Ratio

60.8175

Prob > F

Lower 95\% $\quad$ Upper 95\%

3103.5

2343.5

2343.5

2343.5

Std Err Mean

90.000

30.000

30.000
57.500

30.000
3376.5
2616.5

2616.5

2616.5

2616.5

Lower $95 \%$
2096.4
2098.8
2098.8
1376.9
2098.8

Upper 95\% 4383.6 2861.2
2861.2 2861.2 2838.1 
WSRC-TR-2005-00166

SRNL-RPP-2005-00029

Revision 0

Exhibit A2. Statistical Comparisons of Preparation/Crucible Measurements by Element by Type of Sample

Oneway Analysis of Pb By Simulant/ Preparation/Crucible

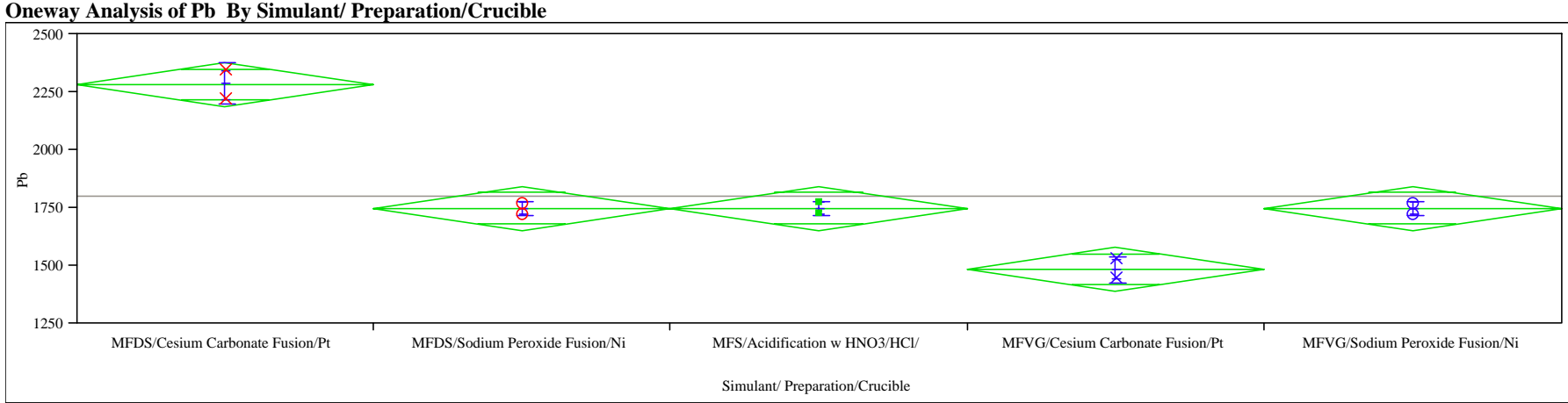

Oneway Anova

Summary of Fit

Rsquare

Adj Rsquare
Root Mean Square Error

Root Mean Square E
Mean of Response

Mean of Response
Observations (or Sum Wgts)

Analysis of Variance

Source

Simulant/ Preparation/Crucible

Error

C. Total

Means for Oneway Anova

Level

MFDS/Cesium Carbonate Fusion/Pt

MFS/Sodium Peroxide Fusion/N/

MFVG/Cesium w HO3/HCl/

MFVG/Sodium Peroxide Fusion/P

St E/Sodium Peroxide Fusion/Ni

ror variance

Means and Std Deviations

Level

MFDS/Cesium Carbonate Fusion/Pt

MFDS/Sodium Peroxide Fusion/N/

MFVG/Cicaton W HNO3/HCl/

MFVG/Sodin Carbonate Fusion/Pt

0.963591

53.00943

10

4
5
9

Sum of Squares

14050.00

14050.00

Numbe

$$
\text { Mean }
$$$$
\begin{array}{r}
\text { Mean } \\
2282.50 \\
1747.50 \\
1747.50 \\
1485.00 \\
1747.50
\end{array}
$$

170140

F Ratio

60.5480

Prob $>$ F

(1)

Std Error

37.483

37.483

37.483

37.483
37.483

Number

$\begin{array}{rr}\text { Mean } & \text { Std Dev } \\ 2282.50 & 88.3883 \\ 1747.50 & 31.8198 \\ 1747.50 & 31.8198 \\ 1485.00 & 56.5685 \\ 1747.50 & 31.8198\end{array}$

Lower 95\%
2186.1
1651.1
1651.1
1388.6
1651.1


Std Err Mean
62.500
22.500
22.500
40.000
22.500

0.0002

Upper 95\%

2378.9

1843.9
1843.9

1843.9
1581.4

1581.4

Lower 95\%

1488.4

1461.6

1461.6

1461.6
Upper 95\%

3076.6

2033.4
2033.4

1993.2 
WSRC-TR-2005-00166

SRNL-RPP-2005-00029

Revision 0

Exhibit A2. Statistical Comparisons of Preparation/Crucible Measurements by Element by Type of Sample

Oneway Analysis of S By Simulant/ Preparation/Crucible

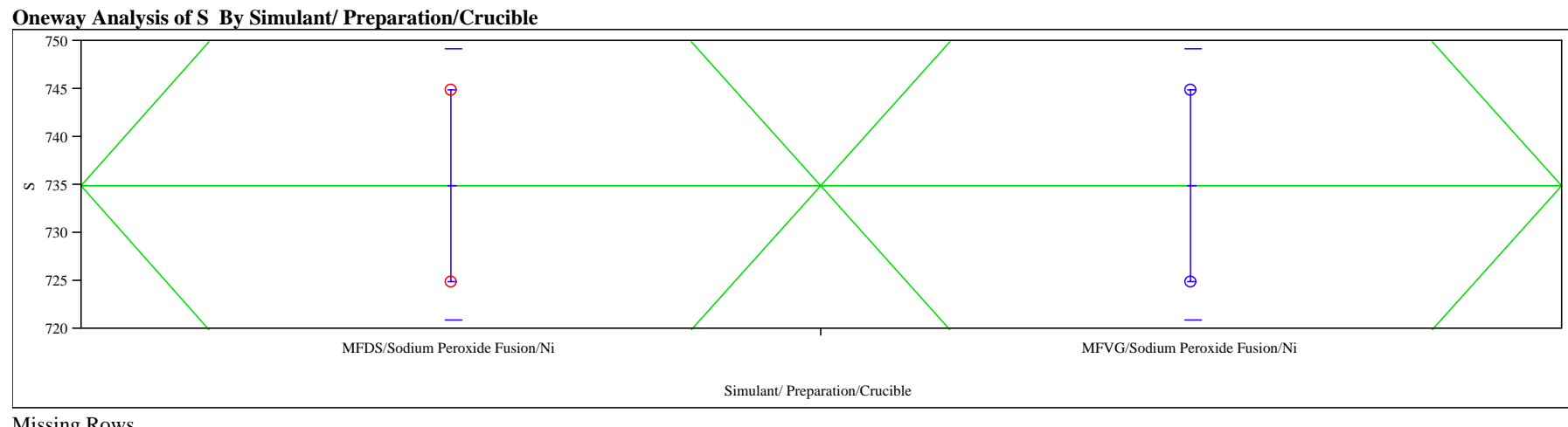

Missing Rows

Summary of Fit

Rsquare

Adj Rsquare

Root Mean Square Error

Mean of Respons

735

Observations (or Sum Wgts)

t Test

MFDS/Sodium Peroxide Fusion/Ni-MFVG/Sodium Peroxide Fusion/Ni

Assuming equal variances

Difference

Std Err Dif

Upper CL Dif

Lower CL Dif

14.142 DF

60.849 Prob $>|t|$

0.95 Prob $<\mathrm{t}$

2
1.0000
0.5000

1.0000
0.5000

Analysis of Variance

Analys

Source
Simulant/ Preparation/Crucible

Error

C. Total

Level

MFDS/Sodium Peroxide Fusion/Ni

MFVG/Sodium Peroxide Fusion/N

Std Error uses a pooled estimate of error variance

Means and Std Deviations

Level

MFDS/Sodium Peroxide Fusion/Ni

MFVG/Sodium Peroxide Fusion/Ni

\begin{tabular}{rrrrrr}
\multicolumn{1}{c}{ DF } & Sum of Squares & Mean Square & F Ratio & Prob > F & \\
1 & 0.00000 & 0.000 & 0.0000 & 1.0000 & \\
2 & 400.00000 & 200.000 & & & \\
3 & 400.00000 & & & & \\
& & & & & \\
Number & Mean & Std Error & Lower 95\% & Upper 95\% \\
2 & 735.000 & 10.000 & 691.97 & 778.03 & \\
2 & 735.000 & 10.000 & 691.97 & 778.03 & \\
& & & & & Upper 95\% \\
& & & & & \\
Number & Mean & Std Dev & Std Err Mean & Lower 95\% & 807.94 \\
2 & 735.000 & 14.1421 & 10.000 & 607.94 & 862.06
\end{tabular}


WSRC-TR-2005-00166

SRNL-RPP-2005-00029

Revision 0

Exhibit A2. Statistical Comparisons of Preparation/Crucible Measurements by Element by Type of Sample

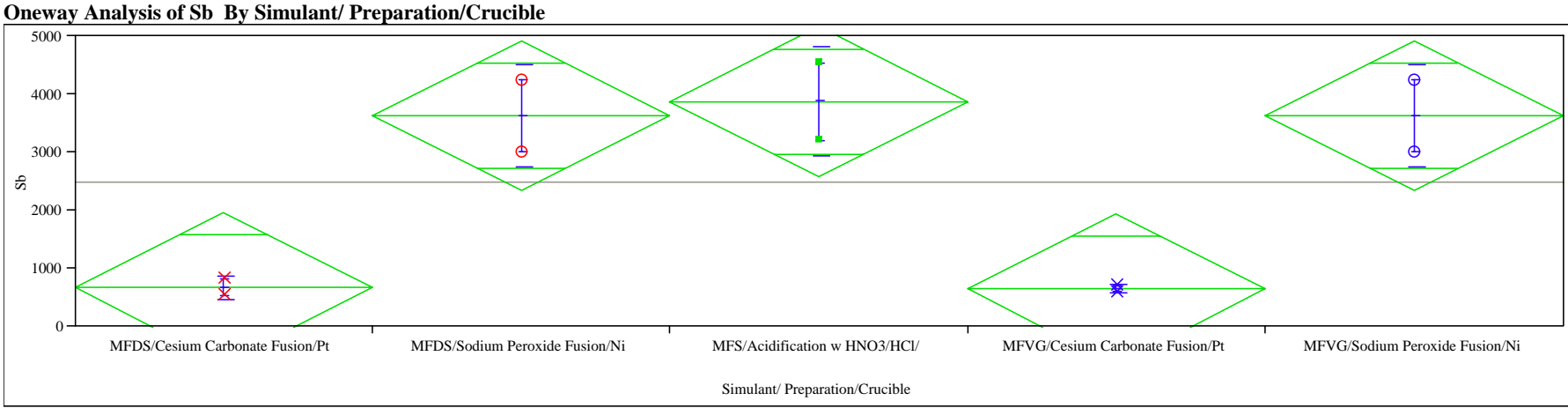

Oneway Anova

Summary of Fit

Rsquare

Adj Rsquare

Root Mean Square Erro

Mean of Response

Observations (or Sum Wgts)

Analysis of Variance

Simulant/ Preparation/Crucible

Error

Means for Oneway Anova

Means

MFDS/Cesium Carbonate Fusion/Pt

MFS/Sodium Peroxide Fusion/N/

MFVG/Cicaton w HNO3/HCI/

MFVG/Sodium Peroxide Fusion/Pt

Stersodium Peroxide Fusion/Ni

ror variance

Means and Std Deviations

Level

MFDS/Cesium Carbonate Fusion/Pt

MFDS/Sodium Peroxide Fusion/ $/$

MFVG/Cinition w HNO3/HCl/

MFVG/Sodin Carbonate Fusion/Pt

imulant/Preparation/Crucible

0.899419

0.81895

707.9819

89.8
10
DF
4
5
9

Sum of Squares 22410962 2506192 24917154

Number

$$
\begin{array}{r}
\text { Mean } \\
674.00 \\
3630.00 \\
3870.00 \\
645.00 \\
3630.00
\end{array}
$$

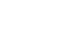

Number

Mean
674.00
3630.00
3870.00
645.00
3630.00

Mean
674.00
3630.00
3870.00
645.00
3630.00

197.990

890.955

933.381

90.510
890.955

Mean Squa
560274
501238


Std Error
500.62
500.62
500.62
500.62
500.62


Std Dev
197.990
890.955
933.381
90.510
890.955

Square

$$
\begin{array}{r}
\text { F Ratio } \\
11.1778
\end{array}
$$

Prob $>$ F

.0104

$\begin{array}{rr}\text { Lower 95\% } & \text { Upper 95\% } \\ -613 & 1960.9 \\ 2343 & 4916.9 \\ 2583 & 5156.9 \\ -642 & 1931.9 \\ 2343 & 4916.9\end{array}$

Std Err Mean

140.00

630.00

660.00
64.00

630.00
2453

11635
12256

1458
11635 
WSRC-TR-2005-00166

SRNL-RPP-2005-00029

Revision 0

Exhibit A2. Statistical Comparisons of Preparation/Crucible Measurements by Element by Type of Sample

Oneway Analysis of Si By Simulant/ Preparation/Crucible

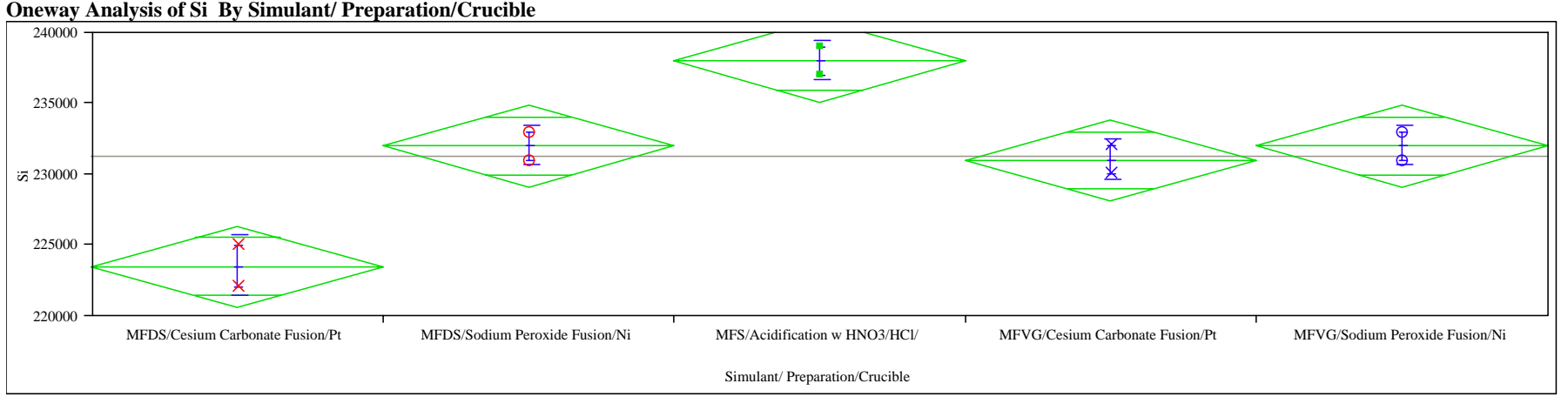

Oneway Anova

\section{Rsquare}

Adj Rsquare

Root Mean Square Erro

Mean of Response

Observations (or Sum Wgts)

Analysis of Variance

Simulant/Preparation/Crucible

C. Total

C. Teans for Oneway Anova

Level

MFDS/Cesium Carbonate Fusion/Pt

MFDS/Sodium Peroxide Fusion/Ni

MFS/Acidification w $\mathrm{HNO} / \mathrm{HCl} /$

MFVG/Cesium Carbonate Fusion/Pt

Std Error uses a pooled estimate of error variance

Means and Std Deviations

Level

MFDS/Cesium Carbonate Fusion/Pt

MFDS/Sodium Peroxide Fusion/Ni

MFS/Acidification w $\mathrm{HNO} / \mathrm{HCl} /$

MFVG/Cesium Carbonate Fusion/Pt

MFVG/Sodium Peroxide Fusion/Ni

0.944715
0.900487
1581.139
231300
10
DF
4
5
9

4
5
9

Sum of Squares

213600000

226100000

Number
2
2
2
2
2

$$
\begin{array}{r}
\text { Mean } \\
223500 \\
232000 \\
238000 \\
231000 \\
232000
\end{array}
$$

Numbe

Mean
223500
232000
238000
231000
232000

Mean Square

$$
\begin{array}{r}
53400000 \\
2500000
\end{array}
$$

F Ratio

Prob $>$ F

Error

Std Error
1118.0
1118.0
1118.0

1118.0

1118.0

Std Dev

Std Dev

1414.21
1414.21

1414.21
1414.21

1414.21
Lower 95\%
220626
229126
235126
228126
Std Err Mean

$$
\begin{array}{r}
\text { Err Mean } \\
1500.0 \\
1000.0 \\
1000.0 \\
1000.0
\end{array}
$$$$
\begin{aligned}
& 1000.0 \\
& 1000.0
\end{aligned}
$$

226374

234874

240874

233874
Lower 95\%
204441
219294
225294
218294


WSRC-TR-2005-00166

SRNL-RPP-2005-00029

Revision 0

Exhibit A2. Statistical Comparisons of Preparation/Crucible Measurements by Element by Type of Sample

Oneway Analysis of Sn By Simulant/Preparation/Crucible

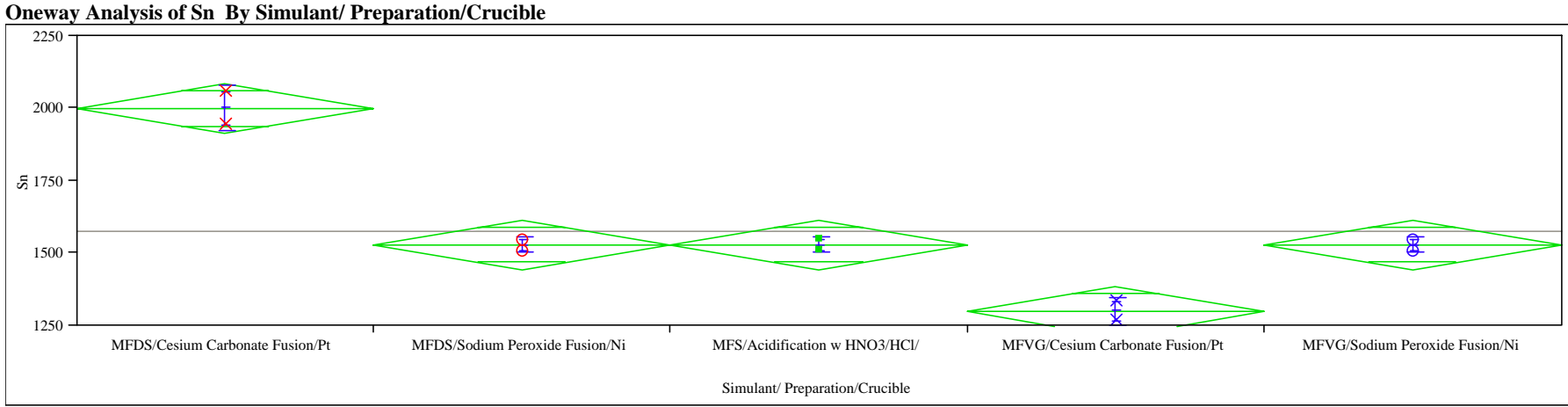

Oneway Anova

Summary of Fit

Rsquare

Adj Rsquare

Root Mean Square

Mean of Response
Observations (or Sum Wgts)

Analysis of Variance

Analysis of

Source

Error

C. Total

Means for Oneway Anova

Level

MFDS/Cesium Carbonate Fusion/Pt

MFS/Sodium Peroxide Fusion/N/

MFVG/Cesiuton w HNO3/HCI/

MFVG/Sodium Peroxide Fusion/Pt

S G/Sodium Peroxide Fusion/Ni

ror variance

Means and Std Deviations

Level

MFDS/Cesium Carbonate Fusion/Pt

MFDS/Sodium Peroxide Fusion/N/

MFVG/Cicaton W HNO3/HCl/

MFVG/Sodin Carbonate Fusion/Pt

0.979644

0.963359

6.69047

578
10

4
5
9

Sum of Squares

24560.00

535460.00
9

Mean Square

131140

F Ratio

Prob $>$ F

Number

Mean
2000.00

2000.00
1530.00

1530.00
1530.00

1530.00

Std Error

33.015

33.015

33.015

33.015
33.015

Number

Mean

2000.00

1530.00

1300.00

1530.00

Std Dev

77.7817

-1
-1

28.2843

28.2843

\section{.}

Lower 95\%

1915.1

1445.1

1445.1

1445.1

55.000

20.000

20.000

20.000
Std Err Mean
0.0002

Upper 95\%

2084.9

1614.9

1614.9

1614.9

Lower 95\%

1301.2

1275.9

1275.9

1275.9
Upper 95\%

2698.8

1784.1
1784.1

1744.7 
WSRC-TR-2005-00166

SRNL-RPP-2005-00029

Revision 0

Exhibit A2. Statistical Comparisons of Preparation/Crucible Measurements by Element by Type of Sample

Oneway Analysis of Sr By Simulant/Preparation/Crucible

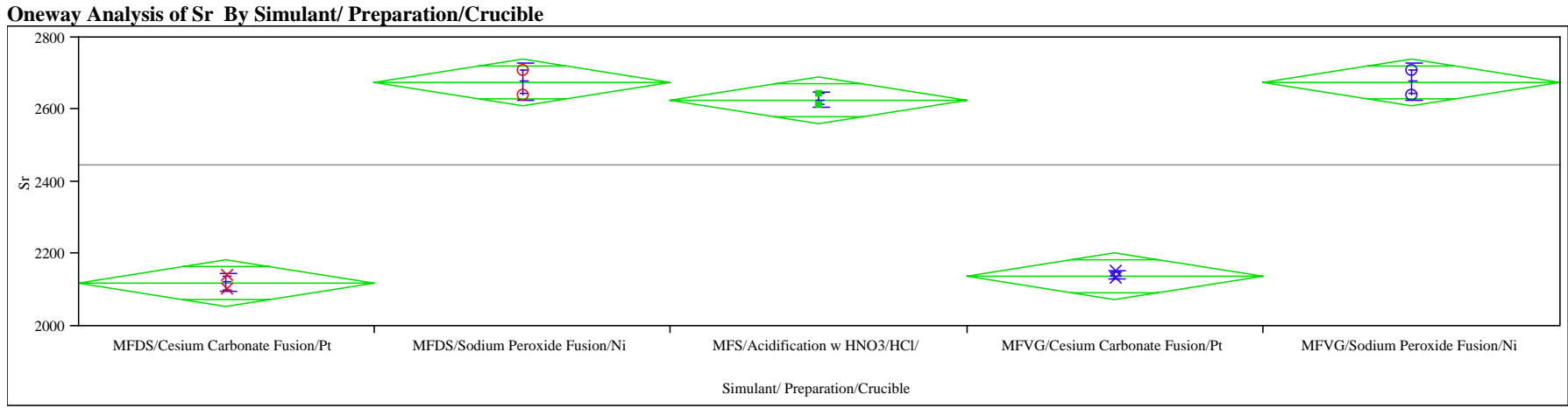

Oneway Anova

Summary of Fit

Rsquare

Adj Rsquare

Root Mean Square Error

Mean of Response

Observations (or Sum Wgts)

Analysis of Variance

Simulant/ Preparation/Crucible

Error

Means for Oneway Anova

Means

MFDS/Cesium Carbonate Fusion/Pt

MFS/Sodium Peroxide Fusion/N/

MFVG/Cesium w HO3/HCl/

MFVG/Sodium Peroxide Fusion/Pt

ST/Sodium Peroxide Fusion/Ni

rror variance

Means and Std Deviations

Level

MFDS/Cesium Carbonate Fusion/Pt

MFDS/Sodium Peroxide Fusion/N/

MFVG/Cicaton w HNO3/HCl/

MFVG/Sodin Carbonate Fusion/Pt

simulant/Preparation//Crucible

0.990662

0.983191

2447

2447
10
DF
4
5
9

Sum of Squares

673660.00

6350.00

Numbe

\section{Mean}

2120.00

2675.00

2625.00

2140.00

Number

Mean
2120.00
2675.00
2625.00
2140.00
2675.00

Mean
2120.00

2675.00

2675.00
Mean Square

168415

Std Error

25.199

25.199

25.199

25.199

Std Dev

28.2843

49.4975

21.2132

14.1421
49.4975 132.6102

Prob $>$ F

$<.000$

$\begin{array}{rr}\text { Lower } 95 \% & \text { Upper } 95 \% \\ 2055.2 & 2184.8 \\ 2610.2 & 2739.8 \\ 2560.2 & 2689.8 \\ 2075.2 & 2204.8 \\ 2610.2 & 2739.8\end{array}$

Std Err Mean

20.000

35.000

15.000

10.000
35.000
Upper 95\% 2374.1
3119.7

3119.7

2815.6

3119.7 
WSRC-TR-2005-00166

SRNL-RPP-2005-00029

Revision 0

Exhibit A2. Statistical Comparisons of Preparation/Crucible Measurements by Element by Type of Sample

Oneway Analysis of Th By Simulant/ Preparation/Crucible

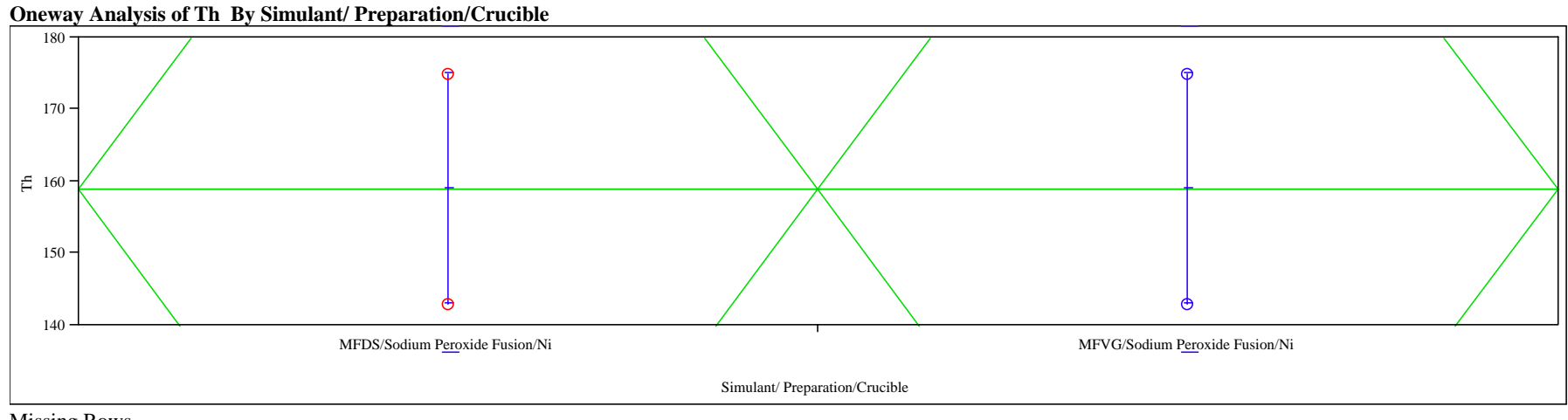

Missing Rows

Summary of Fit

Rsquare

Adj Rsquare

Root Mean Square Error

Mean of Response

742

Observations (or Sum Wgts)

MFDS/Sodium Peroxide Fusion/Ni-MFVG/Sodium Peroxide Fusion/Ni

Assuming equal variances

Difference

Std Err Dif

Upper CL Dif

Lower CL Dif

0.000 t Ratio

22.627 DF

97.358 Prob $>|t|$
-97.358 Prob $>t$

0.95 Prob $<t$

2
1.0000
0.5000

1.0000
0.5000

Analysis of Variance

Analys

Source
Simulant/ Preparation/Crucible

Error

C. Total

Means

MFDS/Sodium Peroxide Fusion/N

MFVG/Sodium Peroxide Fusion/N

Std Error uses a pooled estimate of error variance

Means and Std Deviations

Level

MFDS/Sodium Peroxide Fusion/Ni

MFVG/Sodium Peroxide Fusion/N

\begin{tabular}{rrrrrr}
\multicolumn{1}{c}{ DF } & Sum of Squares & Mean Square & F Ratio & Prob > F & \\
1 & 0.0000 & 0.000 & 0.0000 & 1.0000 & \\
2 & 1024.0000 & 512.000 & & & \\
3 & 1024.0000 & & & & \\
& & & & & \\
Number & Mean & Std Error & Lower 95\% & Upper 95\% \\
2 & 159.000 & 16.000 & 90.158 & 227.84 & \\
2 & 159.000 & 16.000 & 90.158 & 227.84 & \\
& & & & & Upper 95\% \\
& & & & & \\
Number & Mean & Std Dev & Std Err Mean & Lower 95\% & 362.30 \\
2 & 159.000 & 22.6274 & 16.000 & -44.30 & 362.30 \\
2 & 159.000 & 22.6274 & 16.000 & -44.30 &
\end{tabular}


WSRC-TR-2005-00166

SRNL-RPP-2005-00029

Revision 0

Exhibit A2. Statistical Comparisons of Preparation/Crucible Measurements by Element by Type of Sample

Oneway Analysis of Ti By Simulant/ Preparation/Crucible

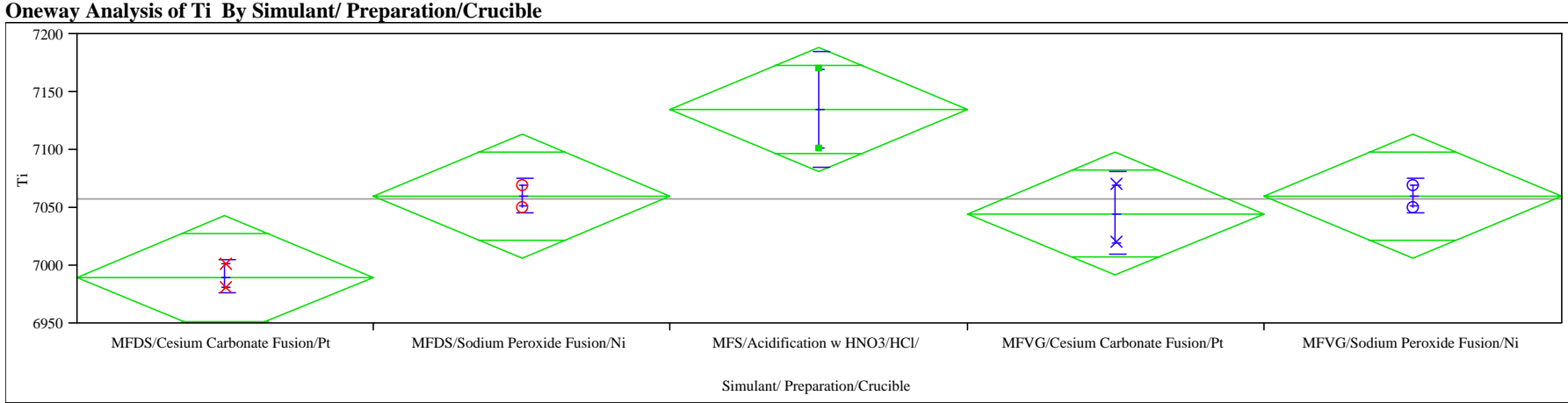

Oneway Anova

Summary of Fit

Rsquare

Adj Rsquare

Root Mean of Response

Mean of Response
Observations (or Sum Wgts)

Analysis of Variance

Source

Simulant/ Preparation/Crucible

Error

C. Total

Means for Oneway Anova

Level

MFDS/Cesium Carbonate Fusion/Pt

MFS/Sodum Peroxide Fusion/Mi

MFVG/Cesiton wNO3/HCI/

MFVG/Sodium Peroxide Fusion/Pt

Sterion

ror variance

Means and Std Deviations

Level

MFDS/Cesium Carbonate Fusion/Pt

MTS/Sodin Peroxide Fusion/N

MFVG/Cicaton w HNO3/HCl/

MFV/Sesinn Carbonate Fusion/Pt
0.833075

0.699534

29.32576

058
10

4
5
9

Sum of Squares 21460.000 4300.000
25760.000

Number

$$
\begin{array}{r}
\text { Mean } \\
6990.00
\end{array}
$$

6990.00

7135.00

7135.00

7060.00

Number

Mean
6990.00
7060.00
7135.00
7045.00
7060.00

Mean
6990.00

7060.00

7045.00

7060.00
Mean Square 5365.00 860.00

Std Error

$$
20.736
$$$$
20.736
$$$$
20.736
$$$$
20.736
$$

Std Dev

14.1421

14.1421

49.4975

14.1421
F Ratio

6.2384 Prob > F

Lower 95\% _ _ Upper 95\%

$\begin{array}{rr}6936.7 & 7043.3 \\ 70067 & 713.3\end{array}$

$\begin{array}{ll}7006.7 & 7113.3 \\ 7081.7 & 7188.3\end{array}$

$\begin{array}{ll}7081.7 & 7188.3 \\ 6991.7 & 7098.3\end{array}$

$\begin{array}{ll}6991.7 & 7098.3 \\ 7006.7 & 7113.3\end{array}$

Std Err Mean

10.000

10.000

35.000

10.000
7117.1

7187.1
7579.7

7579.7

7362.7
7187.1 
WSRC-TR-2005-00166

SRNL-RPP-2005-00029

Revision 0

Exhibit A2. Statistical Comparisons of Preparation/Crucible Measurements by Element by Type of Sample

Oneway Analysis of Tl By Simulant/ Preparation/Crucible

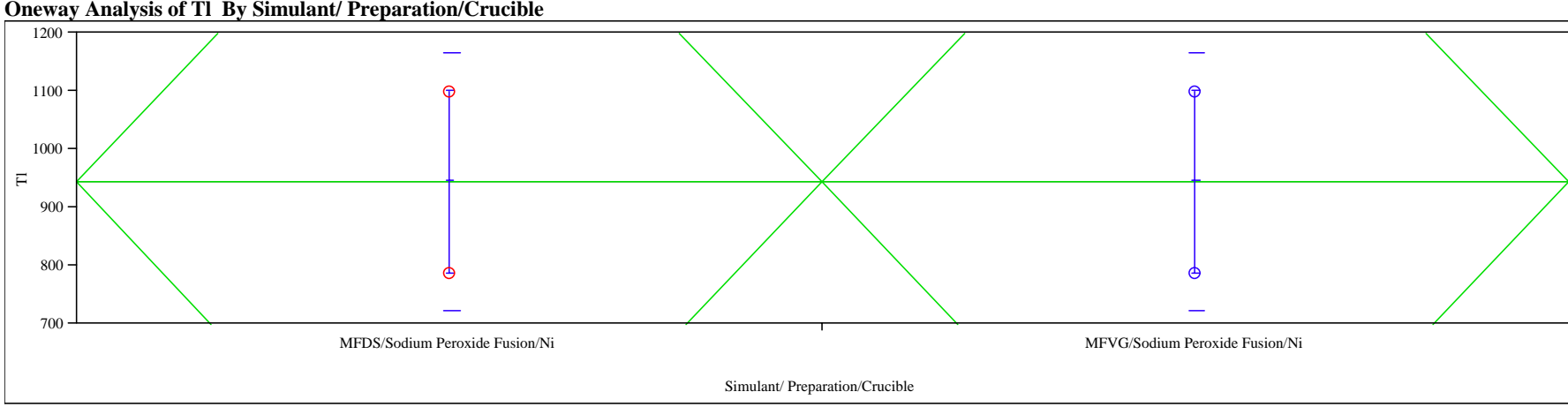

Missing Rows

Summary of Fit

Rsquare

Adj Rsquare

Root Mean Square Error

Mean of Response

Observations (or Sum Wgts)

MFDS/Sodium Peroxide Fusion/Ni-MFVG/Sodium Peroxide Fusion/Ni

Assuming equal variances

Difference

Std Err Dif

Upper CL Dif

Lower CL Dif

0.00 t Ratio

220.62 DF

(9)

0.95 Prob $<\mathrm{t}$

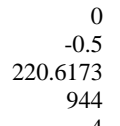

-0.5
6173
944

944
4

Analysis of Variance

Source

Simulant/ Preparation/Crucible

Error

C. Total

Level

MFDS/Sodium Peroxide Fusion/N

GFV/Sodium Peroxide Fusion/

of error variance

Means and Std D

MFDS/Sodium Peroxide Fusion/Ni

2
1.0000
0.5000

0.5000

MFVG/Sodium Peroxide Fusion/Ni

\begin{tabular}{|c|c|c|c|c|c|}
\hline DF & Sum of Squares & Mean Square & F Ratio & Prob $>F$ & \\
\hline 1 & 0.000 & 0.0 & 0.0000 & 1.0000 & \\
\hline 2 & 97344.000 & 48672.0 & & & \\
\hline 3 & 97344.000 & & & & \\
\hline Number & Mean & Std Error & Lower 95\% & Upper 95\% & \\
\hline 2 & 944.000 & 156.00 & 272.79 & 1615.2 & \\
\hline 2 & 944.000 & 156.00 & 272.79 & 1615.2 & \\
\hline Number & Mean & Std Dev & Std Err Mean & Lower 95\% & Upper 95\% \\
\hline 2 & 944.000 & 220.617 & 156.00 & -1038 & 2926.2 \\
\hline 2 & 944.000 & 220.617 & 156.00 & -1038 & 2926.2 \\
\hline
\end{tabular}


WSRC-TR-2005-00166

SRNL-RPP-2005-00029

Revision 0

Exhibit A2. Statistical Comparisons of Preparation/Crucible Measurements by Element by Type of Sample

Oneway Analysis of U By Simulant/ Preparation/Crucible

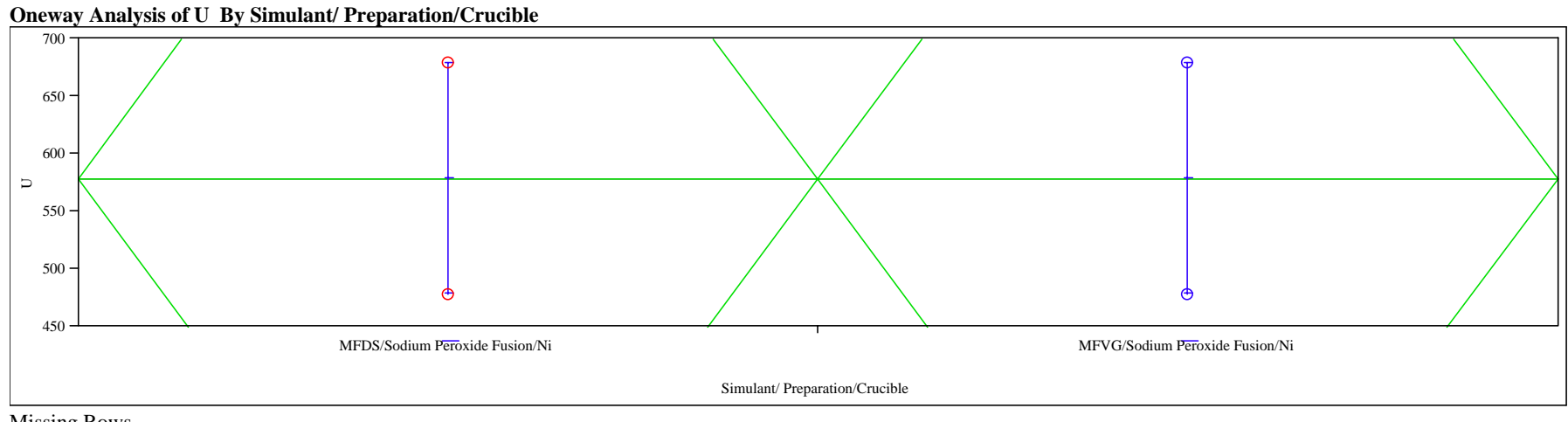

Missing Rows

Summary of Fit

Rsquare

Adj Rsquare

Root Mean Square Error

Mean of Response

285

Observations (or Sum Wgts)

t Test

MFDS/Sodium Peroxide Fusion/Ni-MFVG/Sodium Peroxide Fusion/Ni

Assuming equal variances

Difference

Std Err Dif

Upper CL Dif

Lower CL Dif

142.13 DF

611.53 Prob $>|t|$

0.95 Prob $<\mathrm{t}$

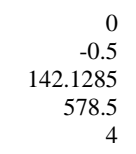

Analysis of Variance

Analysis of Varian

Simulant/ Preparation/Crucible

Error

C. Total

Means for Oneway Anova

Level

MFDS/Sodium Peroxide Fusion/N

MFVG/Sodium Peroxide Fusion/Ni

of error variance

Means and Std Do

MFDS/Sodium Peroxide Fusion/Ni

MFVG/Sodium Peroxide Fusion/Ni

\begin{tabular}{|c|c|c|c|c|c|}
\hline DF & Sum of Squares & Mean Square & F Ratio & Prob $>$ F & \\
\hline 1 & 0.000 & 0.0 & 0.0000 & 1.0000 & \\
\hline 2 & 40401.000 & 20200.5 & & & \\
\hline 3 & 40401.000 & & & & \\
\hline Number & Mean & Std Error & Lower 95\% & Upper 95\% & \\
\hline 2 & 578.500 & 100.50 & 146.08 & 1010.9 & \\
\hline 2 & 578.500 & 100.50 & 146.08 & 1010.9 & \\
\hline Number & Mean & Std Dev & Std Err Mean & Lower 95\% & Upper 95\% \\
\hline 2 & 578.500 & 142.128 & 100.50 & -698.5 & 1855 \\
\hline 2 & 578.500 & 142.128 & 100.50 & -698.5 & 185 \\
\hline
\end{tabular}


WSRC-TR-2005-00166

SRNL-RPP-2005-00029

Revision 0

Exhibit A2. Statistical Comparisons of Preparation/Crucible Measurements by Element by Type of Sample

Oneway Analysis of Y By Simulant/Preparation/Crucible

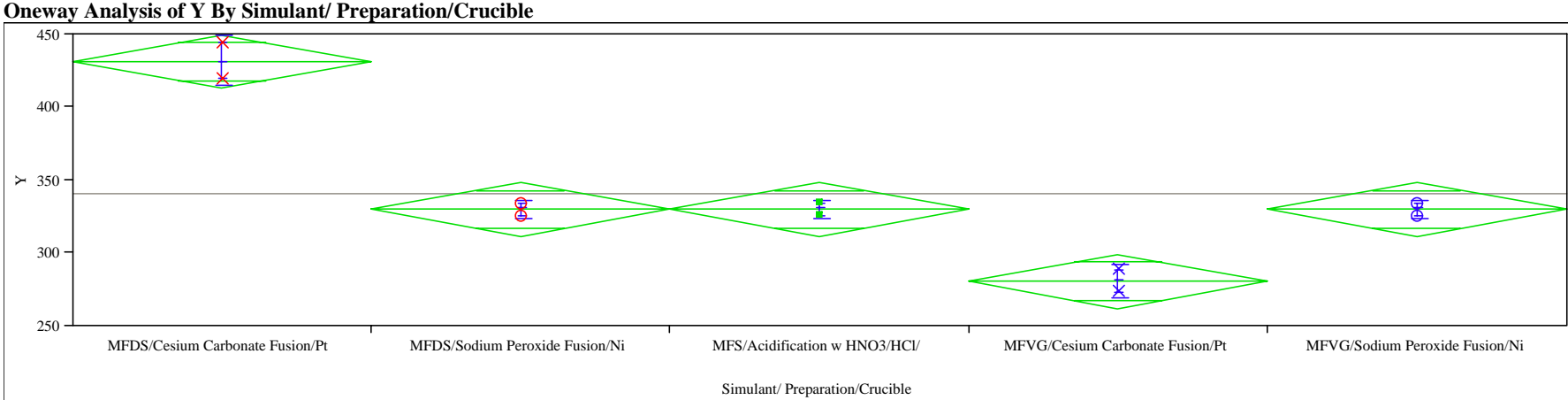

Oneway Anova

Summary of Fit

Rsquare

Adj Rsquare

Root Mean Square Error

Mean of Response

Observations (or Sum Wgts)

Analysis of Variance

Source

Simulant/ Preparation/Crucible

Error

Means for Oneway Anova

Means

Level

MFDS/Cesium Carbonate Fusion/Pt

MFS/Sodium Peroxide Fusion/Ny

MFVG/Cicaton w HNO3/HCI/

MFVG/Sosium Peroxide Fusion/P

S G/Sodium Peroxide Fusion/Ni

or variance

Means and Std Deviations

Level

MFDS/Cesium Carbonate Fusion/Pt

TFS/Sodiam Peroxide Fusion/N/

MFVG/C i C W HNOB/HCl/

MFVG/Sodin Carbonite Fusion/Pt

0.979214

0.962586

10.16366

10
DF
4
5
9

Sum of Squares

24332.400

516.500

Numbe

$$
\begin{array}{r}
\text { Mean } \\
431.500 \\
330.250 \\
330.250 \\
280.750 \\
330.250
\end{array}
$$

Number

Mean
431.500
330.250
330.250
280.750
330.250

Mean
431.500
330.250
330.250
280.750
330.250

Std Dev

6.0104

6.0104

6.0104

6.0104
58.8877

Prob $>$ F

6083.10

103.30

Lower 95\%

413.03

311.78

311.78

262.28
311.78

Std Err Mean

12.000
4.250

4.250
4.250
7.750

7.750
4.250
Upper 95\%

583.97
384.25

384.25

37.22 
WSRC-TR-2005-00166

SRNL-RPP-2005-00029

Revision 0

Exhibit A2. Statistical Comparisons of Preparation/Crucible Measurements by Element by Type of Sample

Oneway Analysis of Zn By Simulant/Preparation/Crucible

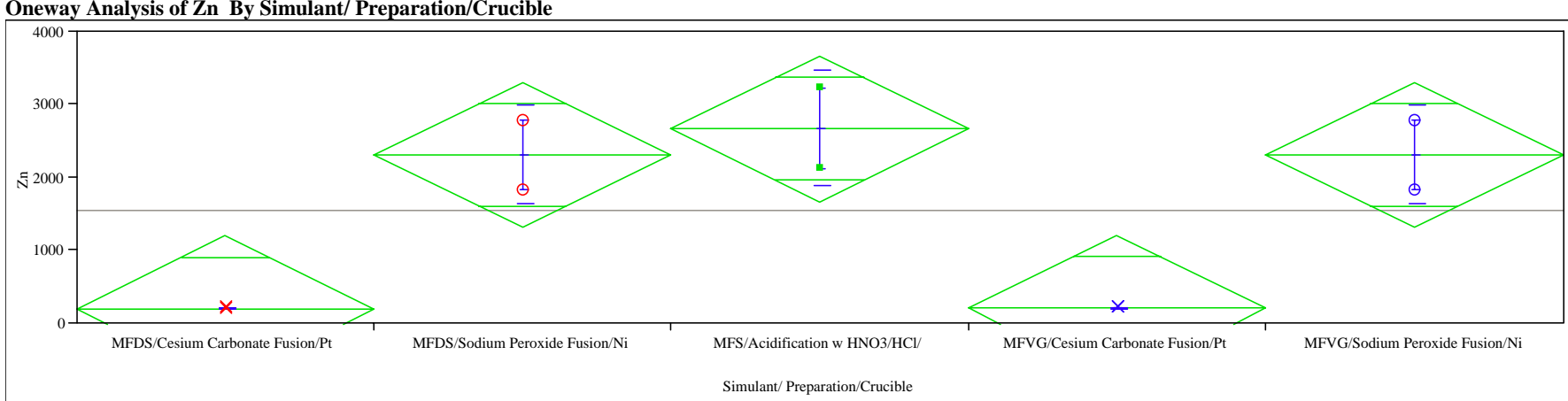

Oneway Anova

Summary of Fit

Rsquare

Adj Rsquare

Mean of Response

Mean of Response
Observations (or Sum Wgts)

Analysis of Variance

Source

Simulant/ Preparation/Crucible

Error

C. Total

Mens for Oneway Anova

Level

MFDS/Cesium Carbonate Fusion/Pt

MFS/Andifict Pexoxide Fusion/N/

MFVG/C siuton wNO3/HCl/

MFVG/Sodium Peroxide Fusion/Pt

S G/Sodium Peroxide Fusion/Ni

or variance

Means and Std Deviations

Level

MFDS/Cesium Carbonate Fusion/Pt

MFSS/Sodian Peroxide Fusion/N/

MFVG/Cinition w HNO3/HCl/

MFVG/Sodin Carbonate Fusion/Pt

\begin{tabular}{|c|c|c|c|c|c|}
\hline $\begin{array}{r}0.888651 \\
0.799572 \\
549.1086 \\
1544 \\
10\end{array}$ & & & & & \\
\hline $\mathrm{DF}$ & Sum of Squares & Mean Square & F Ratio & Prob $>$ F & \\
\hline 4 & 12031861 & 3007965 & 9.9760 & 0.0133 & \\
\hline 5 & 1507601 & 301520 & & & \\
\hline 9 & 13539462 & & & & \\
\hline Number & Mean & Std Error & Lower 95\% & Upper 95\% & \\
\hline 2 & 204.50 & 388.28 & -794 & 1202.6 & \\
\hline 2 & 2315.00 & 388.28 & 1317 & 3313.1 & \\
\hline 2 & 2670.00 & 388.28 & 1672 & 3668.1 & \\
\hline 2 & 215.50 & 388.28 & -783 & 1213.6 & \\
\hline 2 & 2315.00 & 388.28 & 1317 & 3313.1 & \\
\hline Number & Mean & Std Dev & Std Err Mean & Lower 95\% & Upper 95\% \\
\hline 2 & 204.50 & 7.778 & 5.50 & 135 & 274.4 \\
\hline 2 & 2315.00 & 671.751 & 475.00 & -3720 & 8350.4 \\
\hline 2 & 2670.00 & 777.817 & 550.00 & -4318 & 9658.4 \\
\hline 2 & 215.50 & 6.364 & 4.50 & 158 & 272.7 \\
\hline 2 & 2315.00 & 671.751 & 475.00 & -3720 & 8350.4 \\
\hline
\end{tabular}


WSRC-TR-2005-00166

SRNL-RPP-2005-00029

Revision 0

Exhibit A2. Statistical Comparisons of Preparation/Crucible Measurements by Element by Type of Sample

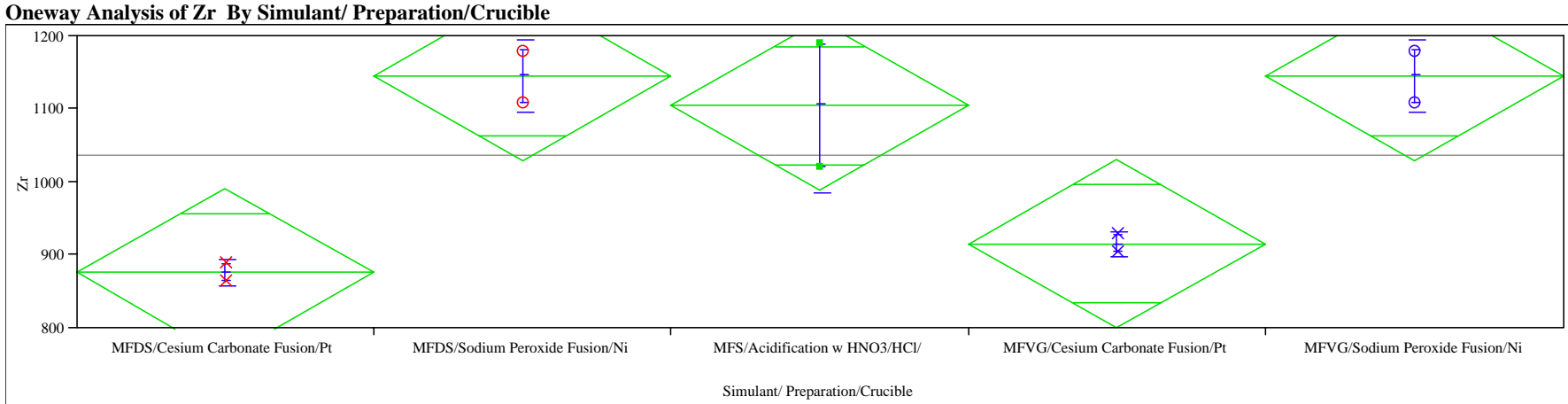

Oneway Anova

Summary of Fit

Rsquare

Adj Rsquare

Root Mean Square Error

Mean of Response

Observations (or Sum Wgts)

Analysis of Variance

Simulant/ Preparation/Crucible

Error

C. Total

Means for Oneway Anova

Level

MFDS/Cesium Carbonate Fusion/Pt

MFS/Sodium Peroxide Fusion/N/

MFVG/Cesiuton w HNO3/HCl/

MFVG/Sodium Peroide Fusion/Pt

S G/Sodium Peroxide Fusion/Ni

ror variance

Means and Std Deviations

Level

MFDS/Cesium Carbonate Fusion/Pt

MFDS/Sodium Peroxide Fusion/N

MFVG/Cicaton w HNO3/HCl/

MFVG/Sodium Cabonase Fusion/Pt
0.872646

0.770764

63.16724
1037.5

10

4
5
9

Sum of Squares

136704.00

19950.50

Numbe

Mean

876.50

1145.00

916.00

1145.00

Number

Mean
876.50
1145.00
1105.00
916.00
1145.00

Mean
876.50

1145.00

916.00

1145.00
Mean Square

34176.

3990.1

Std Error

44.666

44.666

44.666

44.666

Std Dev

17.678

49.497
120.208

120.208
16.971

49.497
F Ratio

8.5652

Prob $>$ F

0.0184

$\begin{array}{rr}\text { Lower 95\% } & \text { Upper 95\% } \\ 761.7 & 991.3 \\ 1030.2 & 1259.8 \\ 990.2 & 1219.8 \\ 801.2 & 1030.8 \\ 1030.2 & 1259.8\end{array}$

Std Err Mean

12.500

35.000

85.000

12.000

Lower $95 \%$
717.67
700.28
24.97
763.53
700.28

Upper 95\%

1035.3

1589.7
2185.0

1068.5 
WSRC-TR-2005-00166

SRNL-RPP-2005-00029

Revision 0

\section{Exhibit A2. Statistical Comparisons of Preparation/Crucible Measurements by Element by Type of Sample}

Type of Sample=Trial

Oneway Analysis of Ag By Simulant/Preparation/Crucible

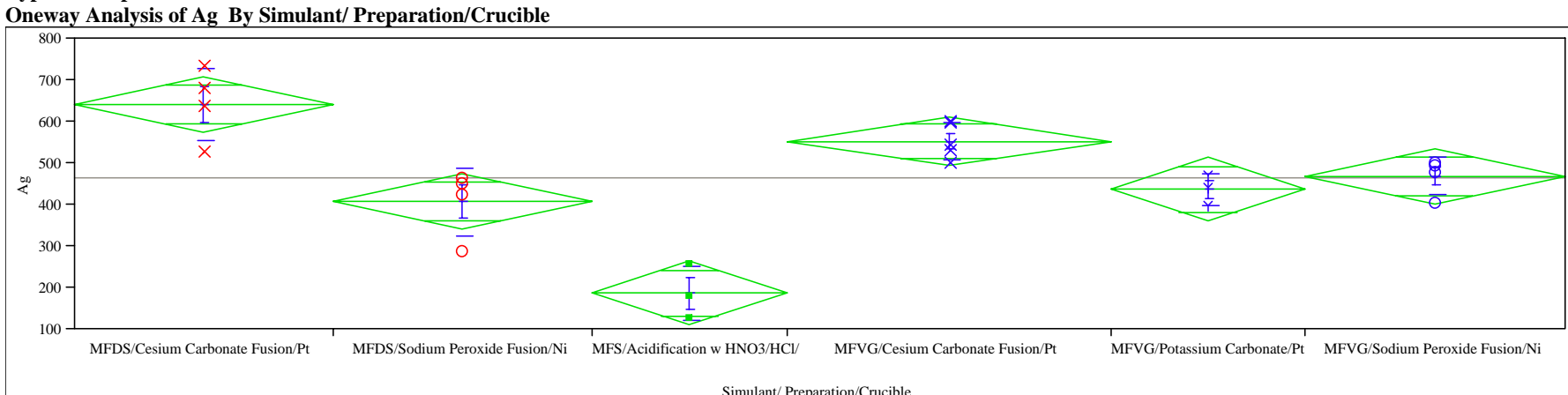

Simulant/Preparation/Crucible

Oneway Anova

Summary of Fit

Rsquare

Adj Rsquare
Root Mean Square Error

Moot Mean of Response

Mean of Response

Analysis of Variance

Source

Simulant/ Preparation/Crucible

Error

Means for Oneway Anova

Means

MFDS/Cesium Carbonate Fusion/Pt

MFS/Aodium Peroxide Fusion/N/

MFVG/Cen w

MFVG/Cesium Carbonate Fusion/Pt

MFVG/Sodium Perolo Fusio

Mrision/Ni

te of error variance

Means and Std Deviation

Level

MFDS/Cesium Carbonate Fusion/Pt

TFS/Aodin Peroxide Fusion/4h/

MFVG/Cicaton w HNO3/HCl/

MFVG/Potassir Cabonate Fusiont

MFVG/Sodsim Peroxide Fusior

0.81714

63.19436

5
17
22

Sum of Squares

412590.99
67889.97

67885.97

Number

Mean

642.750

407.750

187.000

553.200

437.333

(1)

Number

Mean
642.750

642.750
407.750

407.750
187.000

553.200

437.333

469.500
Mean Square

82518.2

F Ratio

20.6630

Prob > F

Std Error
31.597
31.597
36.485
28.261
36.485
31.597



Std Dev
87.2062
81.6267
65.7799
43.8714
37.8197
44.2455

Lower 95\%

576.09
341.09

341.09
110.02

493.57

360.36

Std Err Mean

43.603
40.813

40.813
37.978

37.978

19.620

21.835
22.123
Upper 95\%

781.51
537.64

537.64
350.41

350.41
607.67

607.67
531.28

531.28
539.90 
WSRC-TR-2005-00166

SRNL-RPP-2005-00029

Revision 0

Exhibit A2. Statistical Comparisons of Preparation/Crucible Measurements by Element by Type of Sample

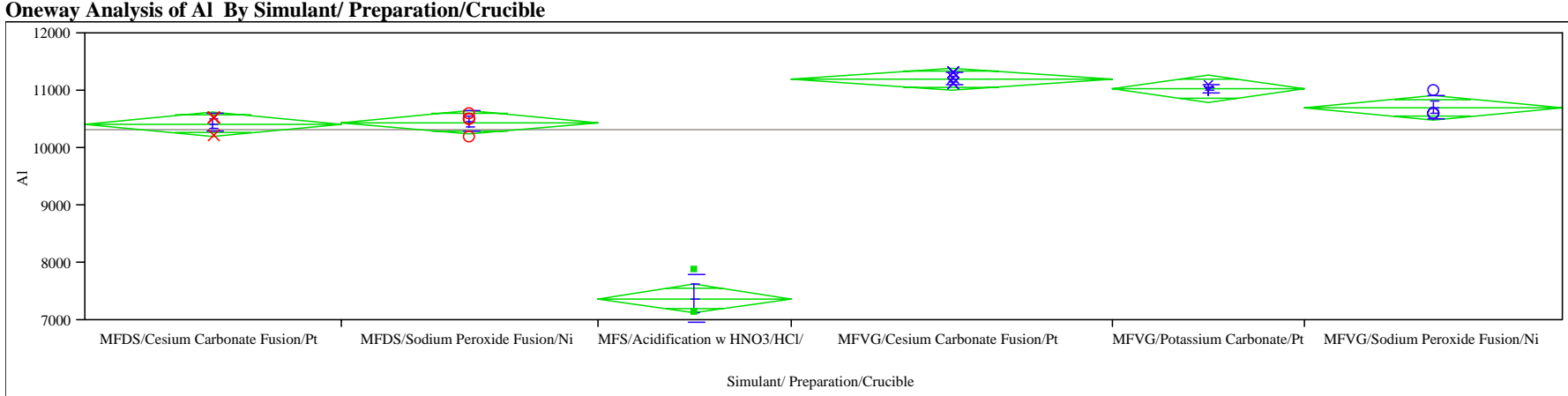

Oneway Anova

Summary of Fit

Rsquare

Adj Rsquare

Root Mean Square Erro

Mean of Response

Observations (or Sum Wgts)

Analysis of Variance

Simulant/Preparation/Crucible

C. Total

Means for Oneway Anova

Level

MFDS/Cesium Carbonate Fusion/Pt

MFDS/Sodium Peroxide Fusion/Ni

MFS/Acidification w $\mathrm{HNO} / \mathrm{HCl}$

MFVG/Cesium Carbonate Fusion/Pt

MFVG/Potassium Carbonate/Pt

MFVG/Sodium Peroxide Fusion/Ni

Std Error uses a pooled estimate of error variance

Means and Std Deviations

Level

MFDS/Cesium Carbonate Fusion/Pt

MFDS/Sodium Peroxide Fusion/Ni

MFS/Acidification w $\mathrm{HNO} / \mathrm{HCl} /$

MFVG/Cesium Carbonate Fusion/P

MFVG/Potassium Carbonate/Pt

MFVG/Sodium Peroxide Fusion/Ni

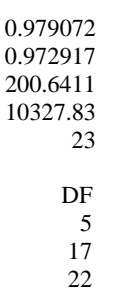

0.979072

0.972917

200.6411

23

DF
5
17
22

Sum of Squares

32017225

32701591

Number
4
4
3
5
3
4

Mean

Mean
10425.0
10450.0

10450.0

7380.0

11200.0

10700.0

Number

Mean
10425.0
10450.0
7380.0
11200.0
11033.3
10700.0
Mean Square
Std Error
100.32
100.32
89.73
89.73
115.84
115.84
Std Dev
150.000
173.205
173.205
424.382
424.382
100.000
100.000
57.735
200.000

6403445

F Ratio

Prob $>$ F

$\begin{array}{rr}\text { Lower } 95 \% & \text { Upper } 95 \% \\ 10213 & 10637 \\ 10238 & 10662 \\ 7136 & 7624 \\ 11011 & 11389 \\ 10789 & 11278 \\ 10488 & 10912 \\ \end{array}$

Std Err Mean

75.00
86.60
245.02
44.72
33.33
100.00

Lower 95\%

10186

10174

6326
11076

11076

10890
10382

Upper 95\%

10664

10726
8434

8434
11324

11324
11177

11177
11018 
WSRC-TR-2005-00166

SRNL-RPP-2005-00029

Revision 0

Exhibit A2. Statistical Comparisons of Preparation/Crucible Measurements by Element by Type of Sample

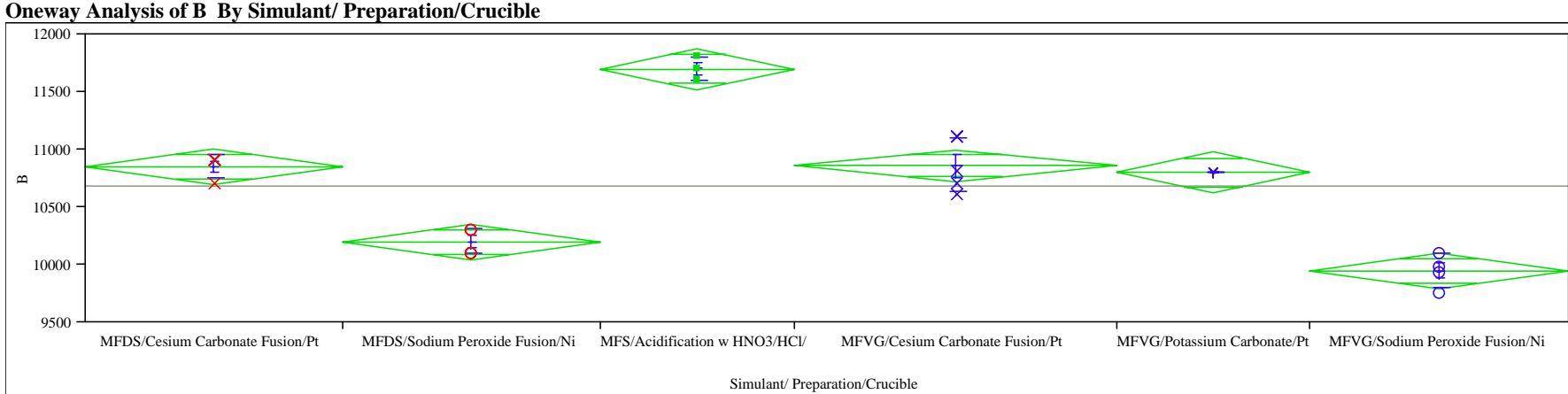

Oneway Anova

Summary of Fit

Rsquare

Adj Rsquare

Root Mean Square Erro

Mean of Response

Observations (or Sum Wgts)

Analysis of Variance

Simulant/ Preparation/Crucible

C. Total

Means for Oneway Anova

Level

MFDS/Cesium Carbonate Fusion/Pt

MFDS/Sodium Peroxide Fusion/Ni

MFS/Acidification w $\mathrm{HNO} / \mathrm{HCl} /$

MFVG/Cesium Carbonate Fusion/Pt

MFVG/Potassium Carbonate/Pt

MFVG/Sodium Peroxide Fusion/Ni

Std Error uses a pooled estimate of error variance

Means and Std Deviations

Level

MFDS/Cesium Carbonate Fusion/Pt

MFDS/Sodium Peroxide Fusion/Ni

MFS/Acidification w $\mathrm{HNO} / \mathrm{HCl} /$

MFVG/Cesium Carbonate Fusion/Pt

MFVG/Potassium Carbonate/Pt

MFVG/Sodium Peroxide Fusion/Ni

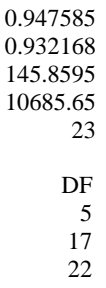

0.947585

0.932168

145.8595

DF
5
17
22

Sum of Squares 6538490.2

6900165.2

Number

$$
\begin{array}{r}
\text { Mean } \\
10850.0 \\
10200.0 \\
11700.0 \\
10860.0 \\
10800.0 \\
9942.5
\end{array}
$$

Number

Mean
10850.0
10200.0
11700.0
10860.0
10800.0
9942.5

9942.5
F Ratio

61.4664

Prob $>$ F

307698

Std Error

72.930

72.930

84.212
65.230

65.230
84.212

84.212
72.930

Std Dev

100.000

115.470
100.000

100.000
230.217

23.000
0.000

141.038

$\begin{array}{rr}\text { Lower 95\% } & \text { Upper 95\% } \\ 10696 & 11004 \\ 10046 & 10354 \\ 11522 & 11878 \\ 10722 & 10998 \\ 10622 & 10978 \\ 9789 & 10096 \\ & \\ \text { Std Err Mean } & \text { Lower } 95 \% \\ 50.00 & 10691 \\ 57.74 & 10016 \\ 57.74 & 11452 \\ 102.96 & 10574 \\ 0.00 & 10800 \\ 70.52 & 9718\end{array}$

11009

10384

11948

11146
10800
10167 
WSRC-TR-2005-00166

SRNL-RPP-2005-00029

Revision 0

Exhibit A2. Statistical Comparisons of Preparation/Crucible Measurements by Element by Type of Sample

Oneway Analysis of Ba By Simulant/ Preparation/Crucible

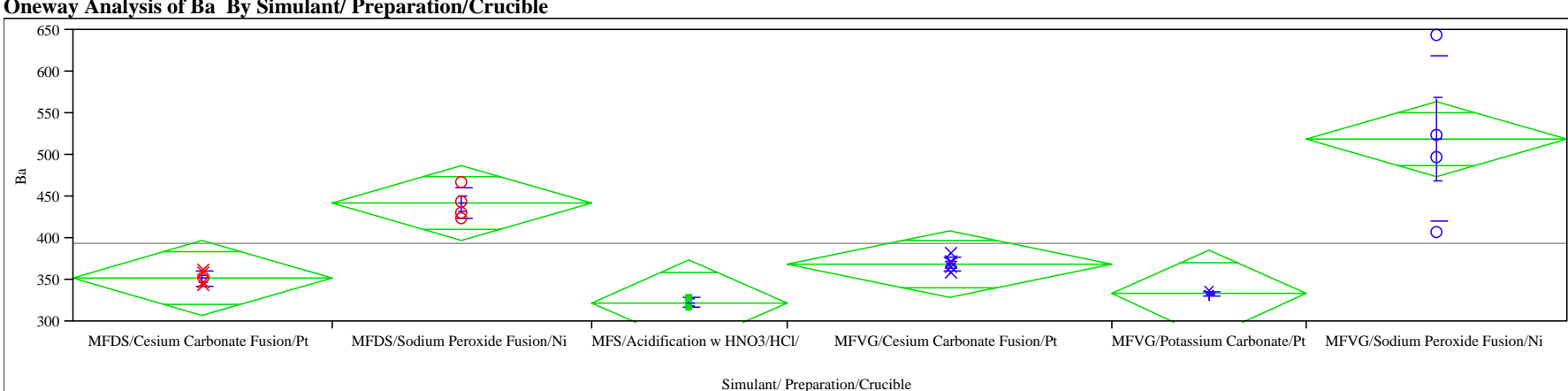

Simulant/Preparation/Crucible

Oneway Anova

Summary of Fit

Rsquare

Adj Rsquare

Root Mean Square Error

Mean of Response

Observations (or Sum Wgts)

Analysis of Variance

Simulant/ Preparation/Crucible

Error

Means for Oneway Anova

Means

Level

MFDS/Cesium Carbonate Fusion/Pt

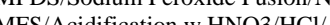

MFVG/Cenion w HNOS/

MFVG/Cesium Carbonate Fusion/Pt

MFVG/Sodium Peroxonate/Pt

MFVG/Sodium Peroxide Fusion/Ni

Mtd Enor uses a poever variance

Means and Std Deviations

Level

MFDS/Cesium Carbonate Fusion/Pt

MFS/Aodiam Peroxide Fusion/N/

MFVG/Cesicion w

MFVG/Cesium Carbonate Fusion/Pt

MFVG/Sodium Peroide Fusion/

Sum of Squares 107649.33 30567.28 138216.61

Number

$$
\begin{array}{r}
\text { Mean } \\
352.000
\end{array}
$$$$
352.000
$$$$
442.000
$$$$
323.333
$$$$
368.600
$$$$
518.750
$$

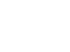

Number

Mean
352.000
442.000
323.333
368.600
333.667
518.750

Mean
352.000
442.000
323.333
368.600
333.667
518.750

$$
\begin{array}{r}
8.8318 \\
18.6548 \\
6.4291 \\
8.5615 \\
2.8868 \\
98.1440
\end{array}
$$

Mean Square
21529
1798.9


Std Error
21.202
21.202
24.482
18.964
24.482
21.202


Std Dev
8.8318
18.6548
6.4291
8.5615
2.8868
98.1440

F Ratio

Prob $>$ F

1798.1

$\begin{array}{rr}\text { Lower 95\% } & \text { Upper 95\% } \\ 307.27 & 396.73 \\ 397.27 & 486.73 \\ 271.68 & 374.99 \\ 328.59 & 408.61 \\ 282.01 & 385.32 \\ 474.02 & 563.48\end{array}$

Std Err Mean

4.416
9.327
3.712
3.829
1.667
49.072

366.05

471.68
339.30

339.30

379.23

340.84
674.92 
WSRC-TR-2005-00166

SRNL-RPP-2005-00029

Revision 0

Exhibit A2. Statistical Comparisons of Preparation/Crucible Measurements by Element by Type of Sample

Oneway Analysis of Ca By Simulant/Preparation/Crucible

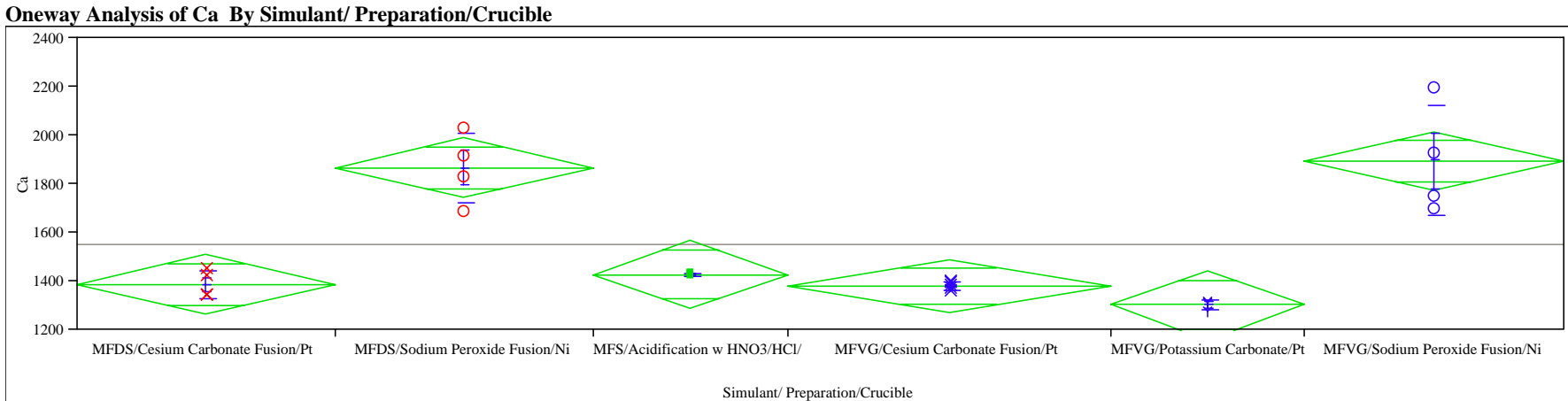

Oneway Anova

Summary of Fit

Rsquare

Adj Rsquare

Root Mean Square Erro

Mean of Response

Observations (or Sum Wgts)

Analysis of Variance

Simulant/ Preparation/Crucible

Error

Means for Oneway Anova

Means

Level

MFDS/Cesium Carbonate Fusion/Pt

MFS/Sodum Peroxide Fusion/Ny

MFVG/Cesimion w HNO3/HCl/

MFVG/Cesium Carbonate Fusion

MFVG/Sodium Peroxide Fusion

Ste

Merror variance

Means and Std Deviations

Level

MFDS/Cesium Carbonate Fusion/Pt

MFS/Aodum Peroxide Fusion/N/

MFVG/Cesical

MFVG/Cosium Cabonate Fusion

MFVG/Sodium Peroxide Fusion/Ni

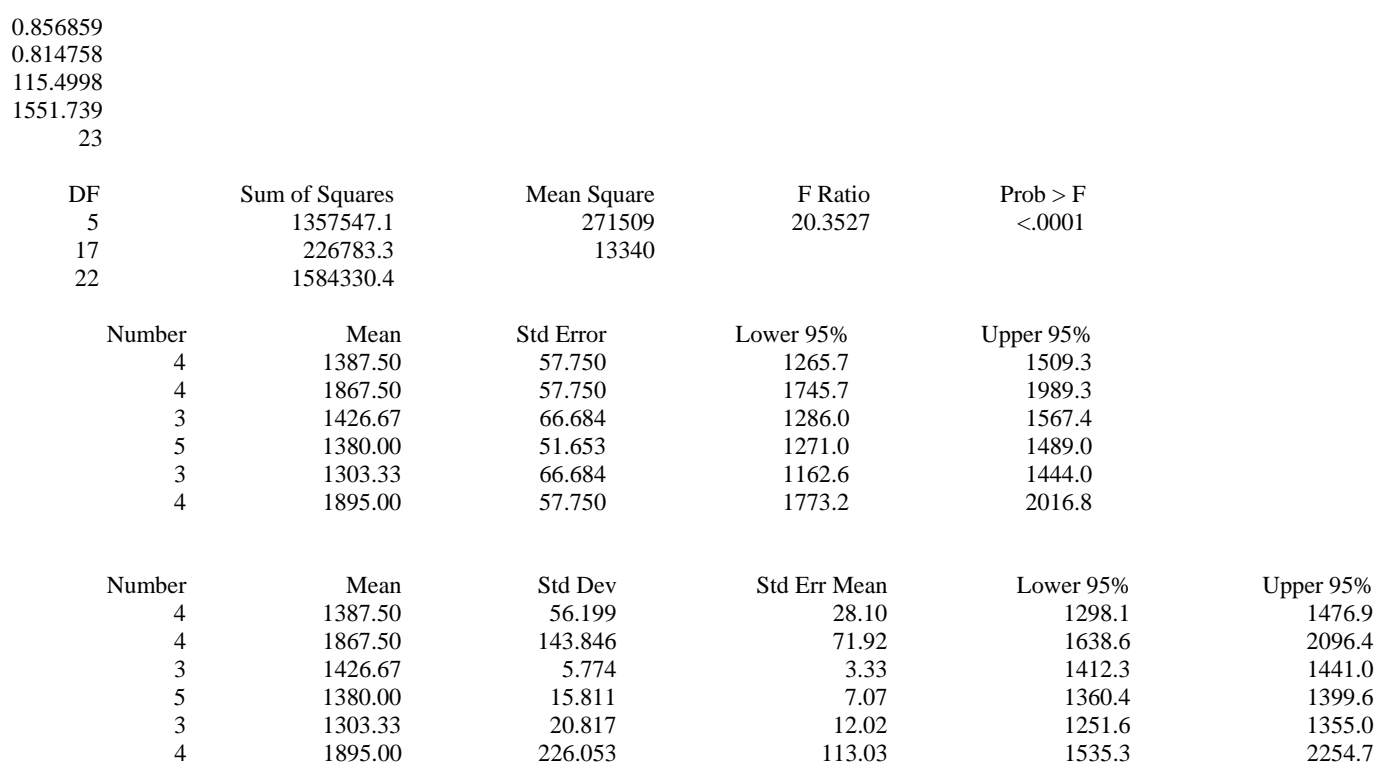


WSRC-TR-2005-00166

SRNL-RPP-2005-00029

Revision 0

Exhibit A2. Statistical Comparisons of Preparation/Crucible Measurements by Element by Type of Sample

Oneway Analysis of Cd By Simulant/ Preparation/Crucible

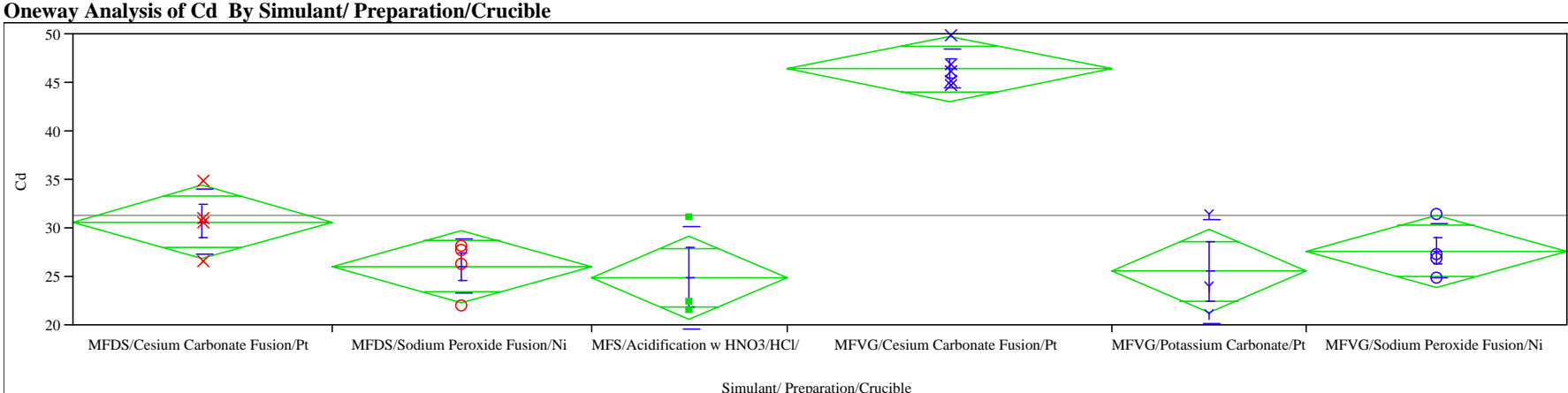

Simulant/ Preparation/Crucible

Oneway Anova

Summary of Fit

Rsquare

Adj Rsquare

Root Mean Square Erro

Mean of Response

Observations (or Sum Wgts)

Analysis of Variance

Simulant/ Preparation/Crucible

Error

Means for Oneway Anova

Means

Level

MFDS/Cesium Carbonate Fusion/Pt

TFS/Acidifin Peroxide Fusion/Ni

MFVG/Celion w HNO3/HCl/

MFVG/Cesium Carbonate Fusion/Pt

MFVG/Sodium Peroxonate Fis

MFVG/Sodium Peroxide Fusion/Ni

Sth Error variance

Means and Std Deviations

Level

MFDS/Cesium Carbonate Fusion/Pt

MFS/Aodum Peroxide Fusion/N/

MFVG/Cesim W HOB/HCI/

MFVG Cesiun Cabonate Fusion

MFVG/Sodium Peroxide Fusion/N

0.843103

3.523596

23

23

5
17
22

Sum of Squares

529.8637

211.0674

Number

Mean
30.7125
26.0875

26.0875

24.9500

46.4600

25.5833
27.7000

Mean

30.7125

26.0875

24.9500

46.4600

25.5833
27.7000
Mean Square
305.973
Error
Std Error
1.7618
1.7618
1.7618
2.0343
1.5758
2.0343
Std Dev
3.39347
2.77920
5.30189
2.06107
5.33206
2.78538

F Ratio

24.6440

Prob $>$ F

Lower $95 \%$
26.995
22.370
20.658
43.135
21.291
23.983


Std Err Mean
1.6967
1.3896
3.0610
0.9217
3.0785
1.3927

Upper 95\%

34.430

29.805

29.242
49.785

49.785
29.875

29.875
31.417
Lower 95\%
25.313
21.665
11.779
11.779
43.901
12.338
23.268
Upper 95\% 36.112 30.510 38.121 49.019 38.829
32.132


WSRC-TR-2005-00166

SRNL-RPP-2005-00029

Revision 0

Exhibit A2. Statistical Comparisons of Preparation/Crucible Measurements by Element by Type of Sample

Oneway Analysis of Ce By Simulant/Preparation/Crucible

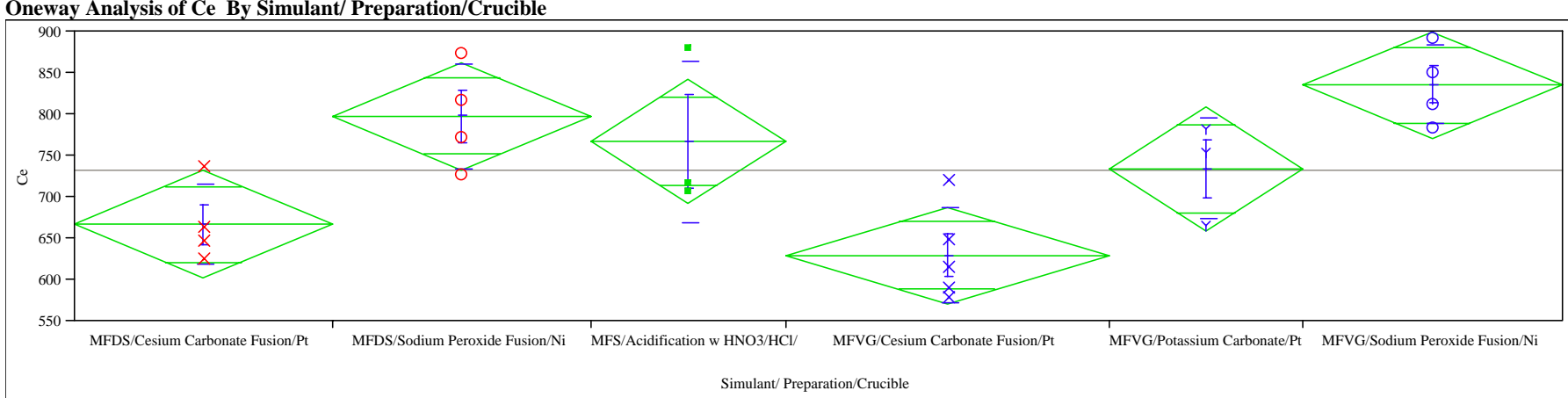

Oneway Anova

Summary of Fit

Rsquare

Adj Rsquare

Root Mean Square Erro

Mean of Response

Observations (or Sum Wgts)

Analysis of Variance

Source

Simulant/ Preparation/Crucible

Error

Means for Oneway Anova

MFDS/Cesium Carbonate Fusion/Pt

MFDS/Sodium Peroxide Fusion/N/

MFV/Acidification w HNO3/HCl/

MFVG/Cesium Carbonate Fusion/

MFVG/Sodium Peabonate/Pt

MFVG/Sodium Peroxide Fusion/Ni

th/ Preparation/Crucible

Means and Std Deviations

Level

MFDS/Cesium Carbonate Fusion/Pt

MFDS/Sodium Peroxide Fusion/N/

MFVG/Cesim W WNOB/HCI

MFVG/Pasium Carbonate Fusion

MFVG/Sodium Peroxide Fusion/Ni

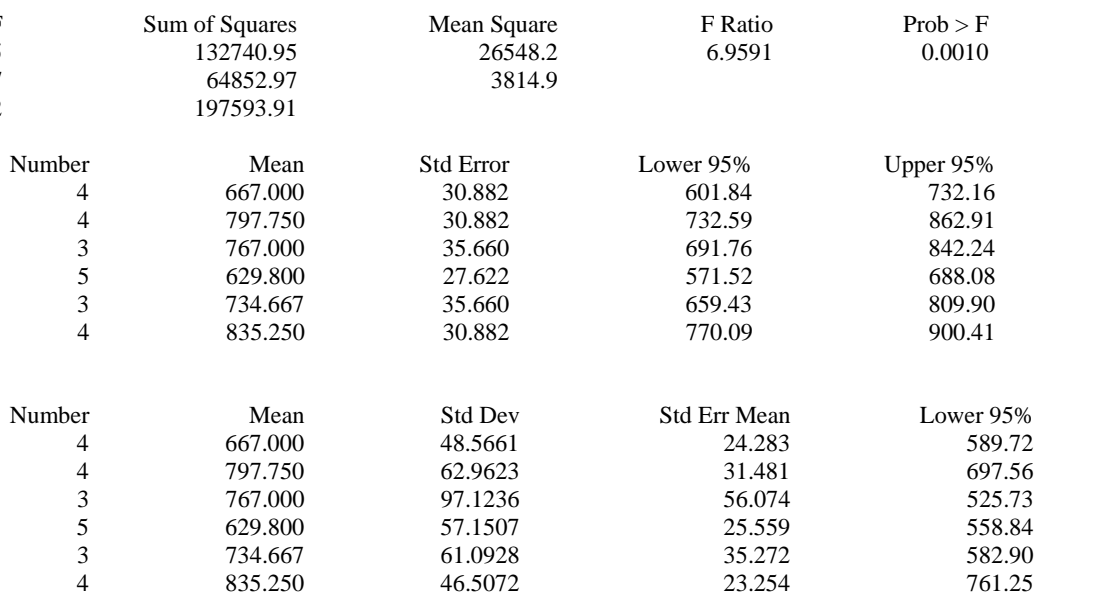

Upper 95\%

744.3

897.9

1008.3
700.8

700.8
886.4

886.4
909.3 
WSRC-TR-2005-00166

SRNL-RPP-2005-00029

Revision 0

Exhibit A2. Statistical Comparisons of Preparation/Crucible Measurements by Element by Type of Sample

Oneway Analysis of $\mathrm{Cr}$ By Simulant/ Preparation/Crucible

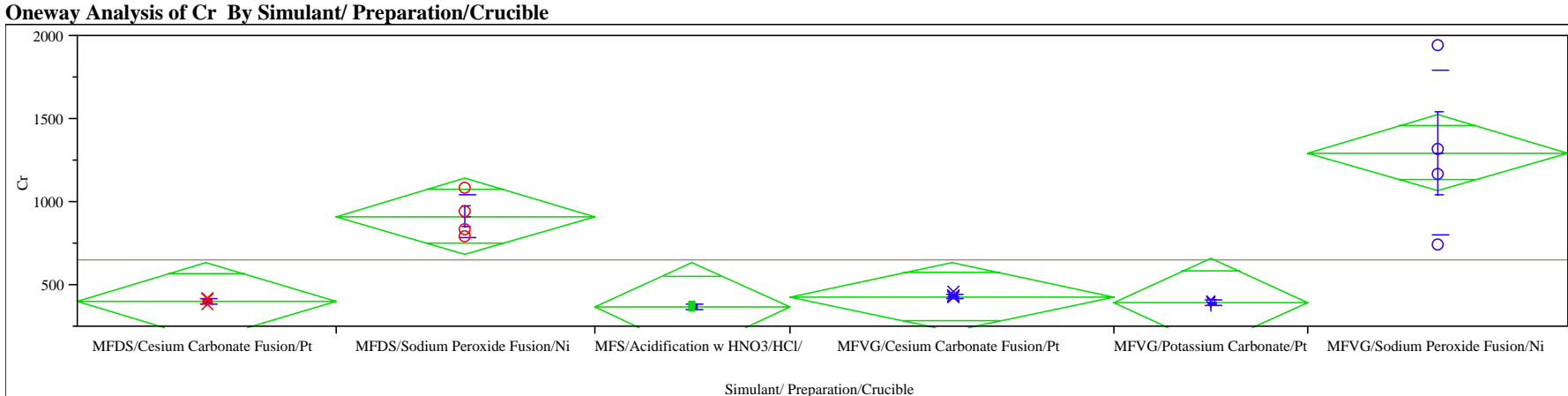

Oneway Anova

Summary of Fit

Rsquare

Adj Rsquare

Root Mean Square Erro

Mean of Response

Observations (or Sum Wgts)

Analysis of Variance

Simulant/ Preparation/Crucible

Error

Means for Oneway Anova

Means

Level

MFDS/Cesium Carbonate Fusion/Pt

MFS/Sodum Peroxide Fusion/Ny

MFVG/Cenion w HNOS/

MFVG/Cesium Carbonate Fusion/Pt

MFVG/Sodium Peroxide Fusion

MFVG/Sodium Peroxide Fusion/Ni

th Preparation/Crucib

Means and Std Deviations

Level

MFDS/Cesium Carbonate Fusion/Pt

MFDS/Sodium Peroxide Fusion/N

MFS/Acidification w HNO3/HCl/

MFVG/Cesium Carbonate Fusion/Pt

TFVG/Potassium Carbonate/Pt

MFVG/Sodium Peroxide Fusion/Ni

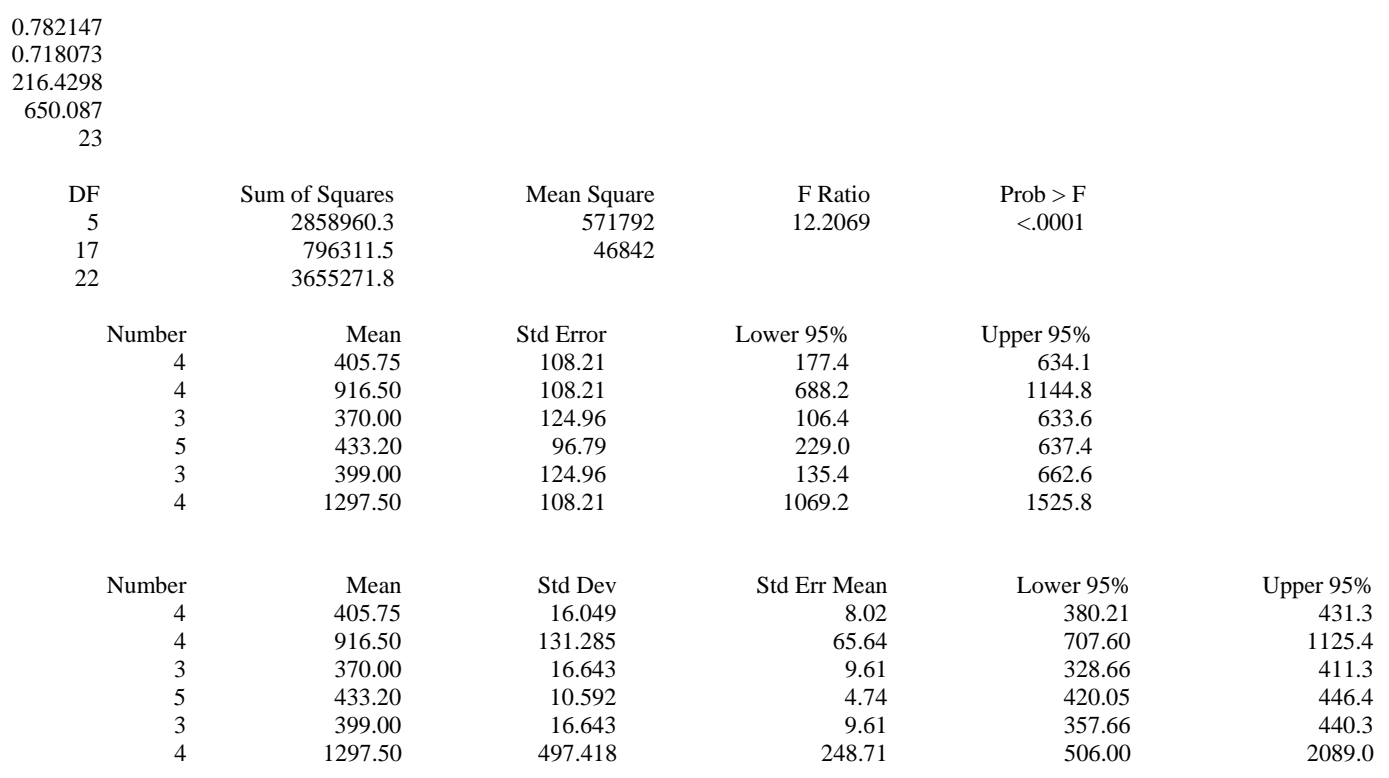


WSRC-TR-2005-00166

SRNL-RPP-2005-00029

Revision 0

Exhibit A2. Statistical Comparisons of Preparation/Crucible Measurements by Element by Type of Sample

Oneway Analysis of Cu By Simulant/Preparation/Crucible

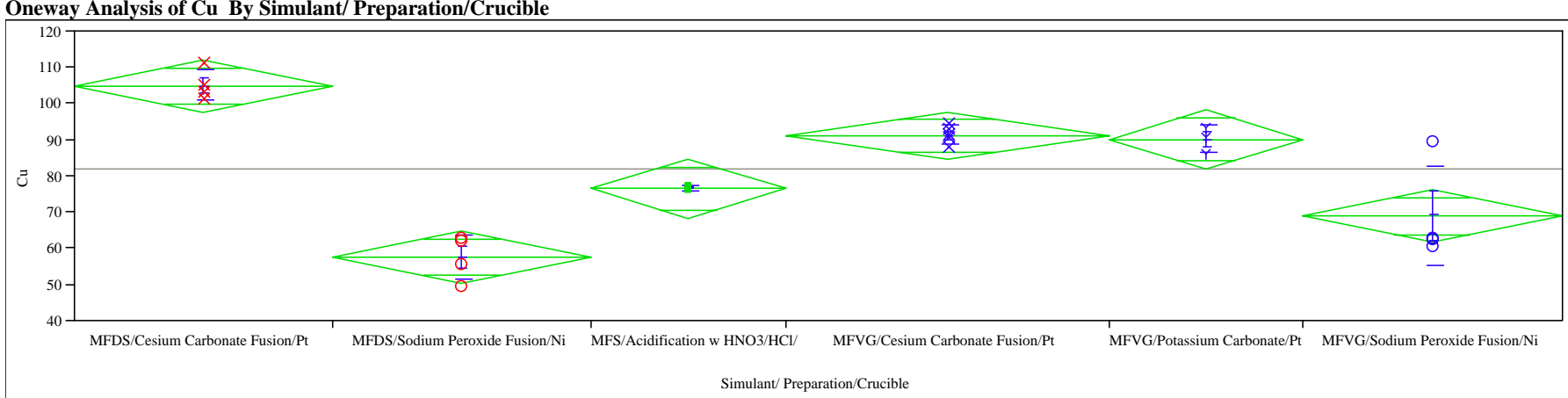

Oneway Anova

Summary of Fit

Rsquare

Adj Rsquare

Root Mean Square Erro

Mean of Response

Observations (or Sum Wgts)

Analysis of Variance

Source

Simulant/ Preparation/Crucible

Error

Means for Oneway Anova

MFDS/Cesium Carbonate Fusion/Pt

MFDS/Sodium Peroxide Fusion/Ni

MFV/Acidication w HNO3/HCl/

MFVG/Cesium Carbonate Fusion/

MFVG/Sodium Perbonate/Pt

MrVisiodium Peroxide Fusion/Ni

Std Error uses a pold Furinge of error variance

Means and Std Deviations

Level

MFDS/Cesium Carbonate Fusion/Pt

MFS/Aodium Peroxide Fusion/N/

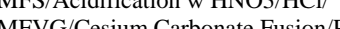

MFVGCsiun Carbonate Fusion/Pt

MFVG/Sodium Peroxide Fusion

Simulant/ Preparation/Crucible

\begin{tabular}{|c|c|c|c|c|c|}
\hline $\begin{array}{r}0.881202 \\
0.846261 \\
6.824446 \\
81.92609 \\
23\end{array}$ & & & & & \\
\hline $\begin{array}{r}\text { DF } \\
5 \\
17 \\
22\end{array}$ & $\begin{array}{r}\text { Sum of Squares } \\
5872.8622 \\
791.7422 \\
6664.6043\end{array}$ & $\begin{array}{r}\text { Mean Square } \\
1174.57 \\
46.57\end{array}$ & $\begin{array}{r}\text { F Ratio } \\
25.2200\end{array}$ & $\begin{array}{r}\text { Prob }>\text { F } \\
<.0001\end{array}$ & \\
\hline $\begin{array}{r}\text { Number } \\
4 \\
4 \\
3 \\
5 \\
3 \\
4\end{array}$ & $\begin{array}{r}\text { Mean } \\
105.000 \\
57.700 \\
76.600 \\
91.320 \\
90.267 \\
69.075\end{array}$ & $\begin{array}{r}\text { Std Error } \\
3.4122 \\
3.4122 \\
3.9401 \\
3.0520 \\
3.9401 \\
3.4122\end{array}$ & $\begin{array}{r}\text { Lower } 95 \% \\
97.801 \\
50.501 \\
68.287 \\
84.881 \\
81.954 \\
61.876\end{array}$ & $\begin{array}{r}\text { Upper } 95 \% \\
112.20 \\
64.90 \\
84.91 \\
97.76 \\
98.58 \\
76.27\end{array}$ & \\
\hline $\begin{array}{r}\text { Number } \\
4 \\
4 \\
3 \\
5 \\
3 \\
4\end{array}$ & $\begin{array}{r}\text { Mean } \\
105.000 \\
57.700 \\
76.600 \\
91.320 \\
90.267 \\
69.075\end{array}$ & $\begin{array}{r}\text { Std Dev } \\
4.3205 \\
6.2059 \\
0.5292 \\
2.4509 \\
3.5572 \\
13.7878\end{array}$ & $\begin{array}{r}\text { Std Err Mean } \\
2.1602 \\
3.1030 \\
0.3055 \\
1.0961 \\
2.0537 \\
6.8939\end{array}$ & $\begin{array}{r}\text { Lower } 95 \% \\
98.125 \\
47.825 \\
75.286 \\
88.277 \\
81.430 \\
47.136\end{array}$ & $\begin{array}{r}\text { Upper } 95 \% \\
111.87 \\
67.57 \\
77.91 \\
94.36 \\
99.10 \\
91.01\end{array}$ \\
\hline
\end{tabular}


WSRC-TR-2005-00166

SRNL-RPP-2005-00029

Revision 0

Exhibit A2. Statistical Comparisons of Preparation/Crucible Measurements by Element by Type of Sample

Oneway Analysis of Fe By Simulant/Preparation/Crucible

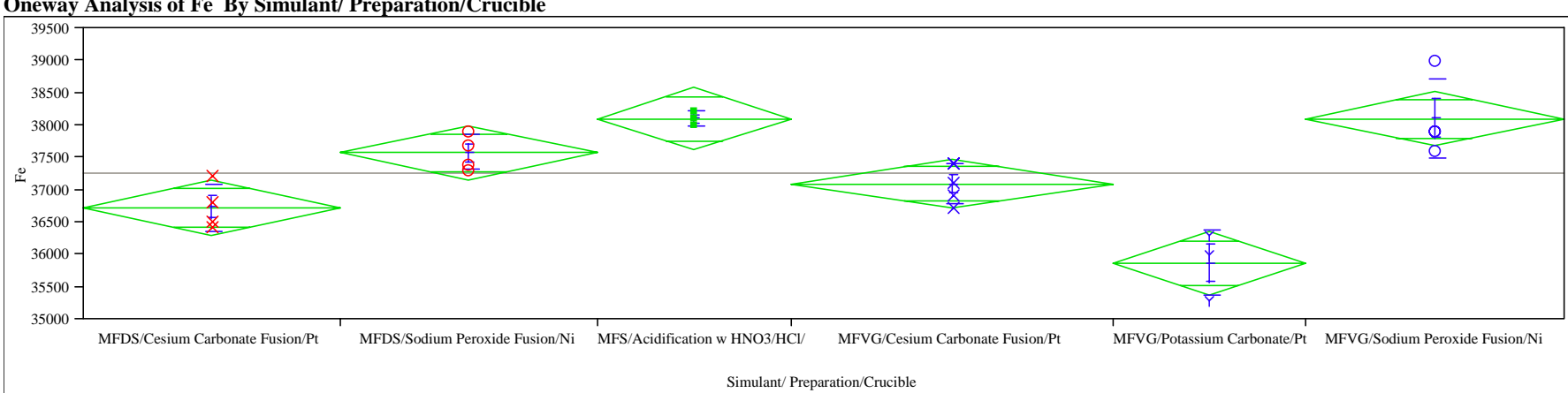

Oneway Anova

Summary of Fit

Rsquare

Adj Rsquare

Root Mean Square Erro

Mean of Response

Observations (or Sum Wgts)

Analysis of Variance

Simulant/ Preparation/Crucible

Error

Means for Oneway Anova

Means

Level

MFDS/Cesium Carbonate Fusion/Pt

MFS/Sodum Peroxide Fusion/M

MFVG/Cesiction w HNO3/HCl/

MFVG/Cesium Carbonate Fusion/Pt

MFVG/Sodium Peroxonate Fusio

MFVG/Soditum Peroxide Fusion/Ni

nt Preparation/Crucible

Means and Std Deviations

Level

MFDS/Cesium Carbonate Fusion/Pt

MFDS/Sodium Peroxide Fusion/N

MFVG/Cesition w HNO3/HCI/

MFVG Cosium Carbonate Fusiont

MFVG/Sodium Peroxide Fusion/N

0.82258
0.770397
397.1714
37260.87
23

DF
5
17
22

\begin{tabular}{|c|c|c|c|c|c|}
\hline & $\begin{array}{r}\text { Sum of Squares } \\
12433116 \\
2681667 \\
15114783\end{array}$ & $\begin{array}{r}\text { Mean Square } \\
2486623 \\
157745\end{array}$ & $\begin{array}{c}\text { F Ratio } \\
15.7636\end{array}$ & $\begin{array}{r}\text { Prob }>\text { F } \\
<.0001\end{array}$ & \\
\hline $\begin{array}{r}\text { Number } \\
4 \\
4 \\
3 \\
5 \\
3 \\
4\end{array}$ & $\begin{array}{r}\text { Mean } \\
36725.0 \\
37575.0 \\
38100.0 \\
37100.0 \\
35866.7 \\
38100.0\end{array}$ & $\begin{array}{r}\text { Std Error } \\
198.59 \\
198.59 \\
229.31 \\
177.62 \\
229.31 \\
198.59\end{array}$ & $\begin{array}{r}\text { Lower 95\% } \\
36306 \\
37156 \\
37616 \\
36725 \\
35383 \\
37681\end{array}$ & $\begin{array}{r}\text { Upper } 95 \% \\
37144 \\
37994 \\
38584 \\
37475 \\
36350 \\
38519\end{array}$ & \\
\hline $\begin{array}{r}\text { Number } \\
4 \\
4 \\
3 \\
5 \\
3 \\
4\end{array}$ & $\begin{array}{r}\text { Mean } \\
36725.0 \\
37575.0 \\
38100.0 \\
37100.0 \\
35866.7 \\
38100.0\end{array}$ & $\begin{array}{l}\text { Std Dev } \\
359.398 \\
275.379 \\
100.000 \\
308.221 \\
513.160 \\
616.441\end{array}$ & $\begin{array}{r}\text { Std Err Mean } \\
179.70 \\
137.69 \\
57.74 \\
137.84 \\
296.27 \\
308.22\end{array}$ & $\begin{array}{r}\text { Lower } 95 \% \\
36153 \\
37137 \\
37852 \\
36717 \\
34592 \\
37119\end{array}$ & $\begin{array}{r}\text { Upper } 95 \% \\
37297 \\
38013 \\
38348 \\
37483 \\
37141 \\
39081\end{array}$ \\
\hline
\end{tabular}


WSRC-TR-2005-00166

SRNL-RPP-2005-00029

Revision 0

Exhibit A2. Statistical Comparisons of Preparation/Crucible Measurements by Element by Type of Sample

Oneway Analysis of K By Simulant/Preparation/Crucible

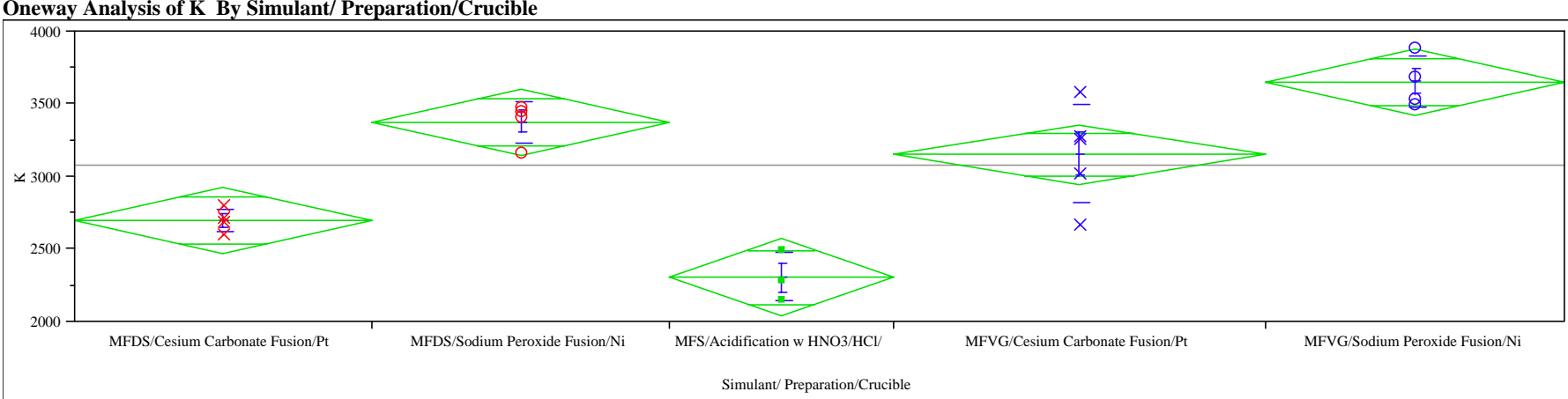

Missing Rows

Revay Anova

Summary of Fit

Rsquare

Adj Rsquare

Root Mean Square E

Mean of Response

Observations (or Sum Wgts)

Analysis of Variance

Simulant/ Preparation/Crucible

C. Total

Means for Oneway Anova

Level

MFDS/Cesium Carbonate Fusion/Pt

MFS/Sodium Peroxide Fusion/N/

MFVG/Cesiun $\mathrm{W}$ -

MFVG/Sodium Perbonate Fusion/Pt

S G/Sodium Peroxide Fusion/Ni

or variance

Means and Std Deviation

Level

MFDS/Cesium Carbonate Fusion/Pt

MDS/Sodiam Peroxide Fusion/N

MFVG/Ciniton W HNO3/HCl/

MFVG/Sodin Carbonate Fusion/Pt

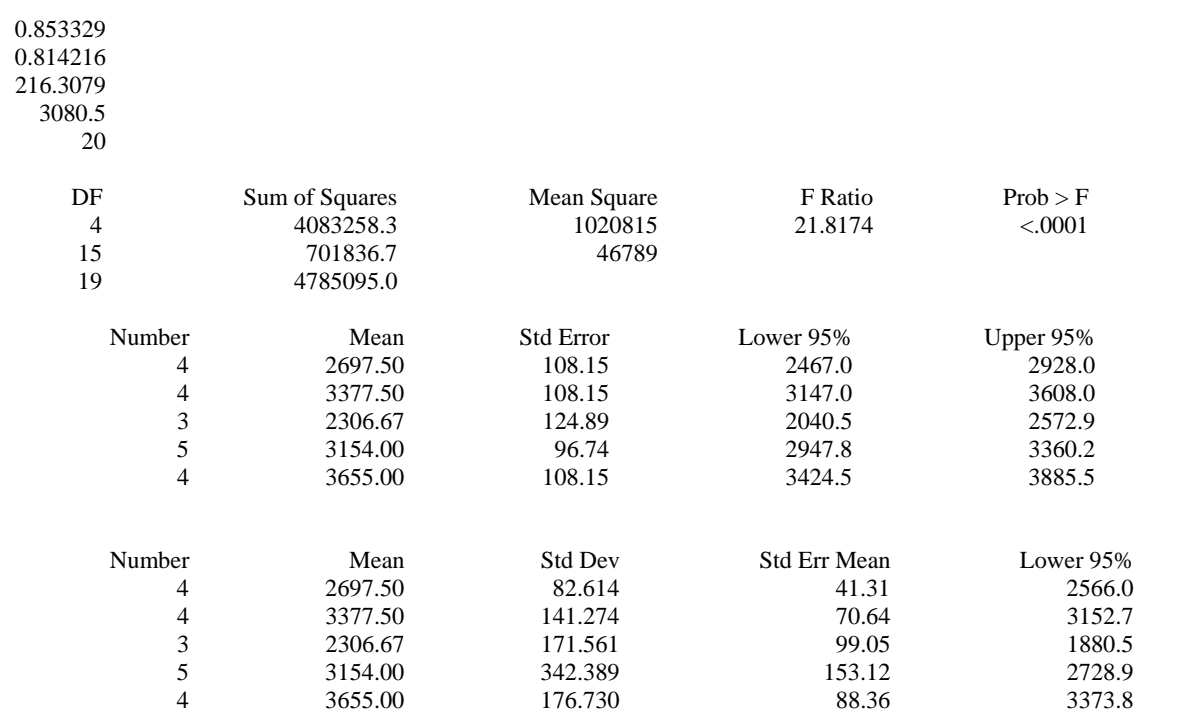

Upper 95\%

2829.0

3602.3

2732.8
3579.1

3936.2 
WSRC-TR-2005-00166

SRNL-RPP-2005-00029

Revision 0

Exhibit A2. Statistical Comparisons of Preparation/Crucible Measurements by Element by Type of Sample

Oneway Analysis of La By Simulant/ Preparation/Crucible

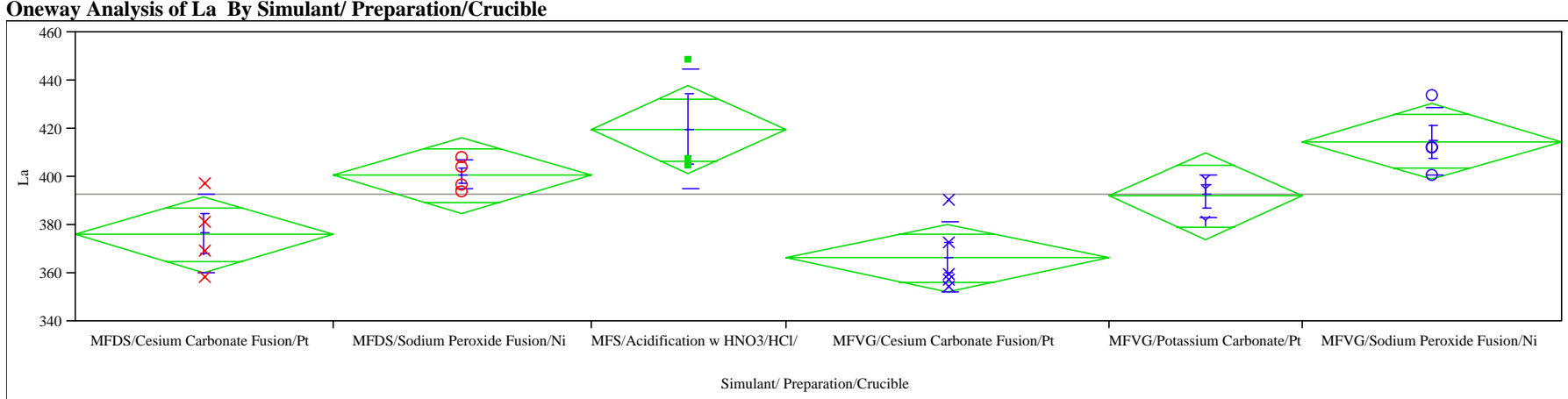

Oneway Anova

Summary of Fit

Rsquare

Adj Rsquare

Root Mean Square Erro

Mean of Response

Observations (or Sum Wgts)

Analysis of Variance

Source

Simulant/ Preparation/Crucible

Error

Means for Oneway Anova

MFDS/Cesium Carbonate Fusion/Pt

MFDS/Sodium Peroxide Fusion/N/

MFS/Acidication w HNO3/HCl/

MFVG/Cesium Carbonate Fusion/

MFVG/Sodium Perbonate/Pt

MFVG/Sodium Peroxide Fusion/Ni

th/ Preparation/Crucible

Means and Std Deviations

Level

MFDS/Cesium Carbonate Fusion/Pt

MFS/Acidium Peroxide Fusion/N/

MFVG/Cesicalon WNO3/HCl/

MFV/Cesium Carbonate Fusion/Pt

MFVG/Sodium Peroxide Fusion

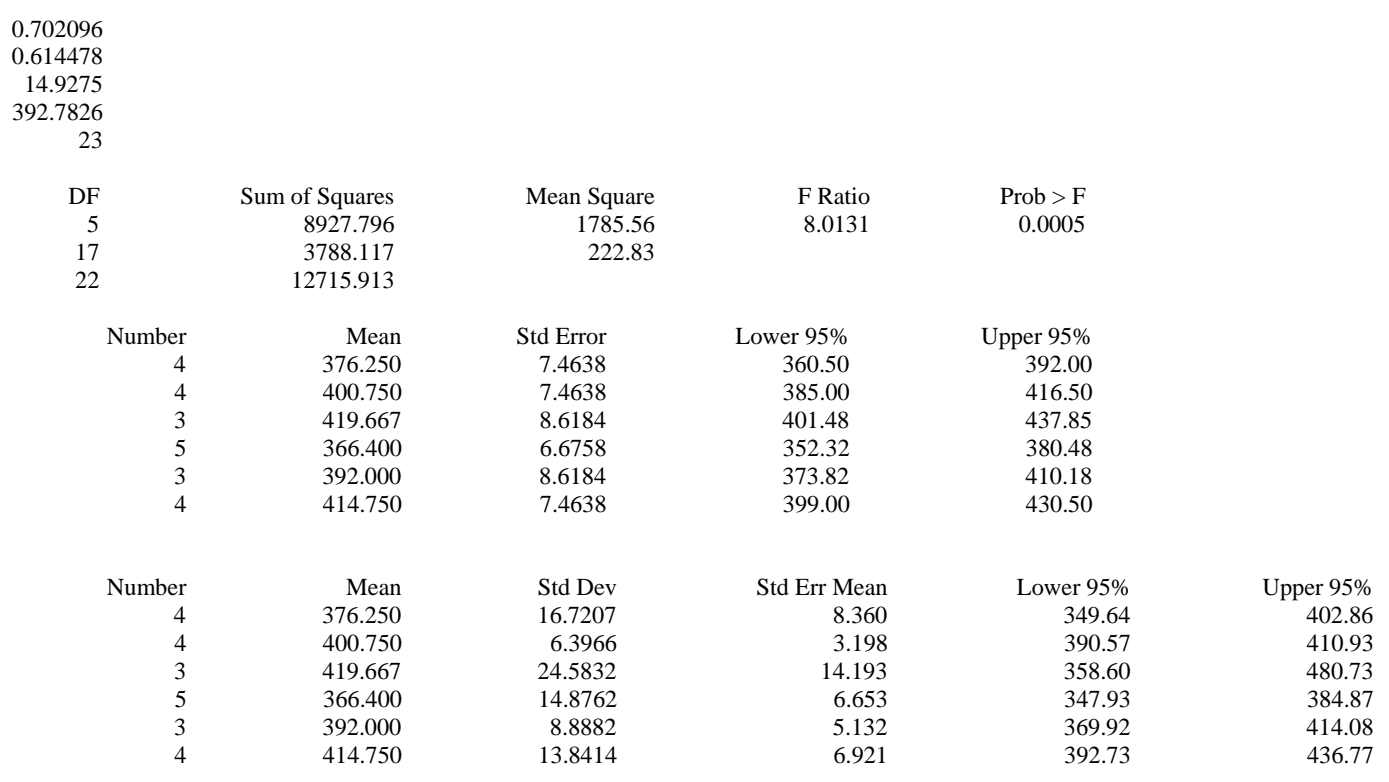


WSRC-TR-2005-00166

SRNL-RPP-2005-00029

Revision 0

Exhibit A2. Statistical Comparisons of Preparation/Crucible Measurements by Element by Type of Sample

Oneway Analysis of Li By Simulant/ Preparation/Crucible

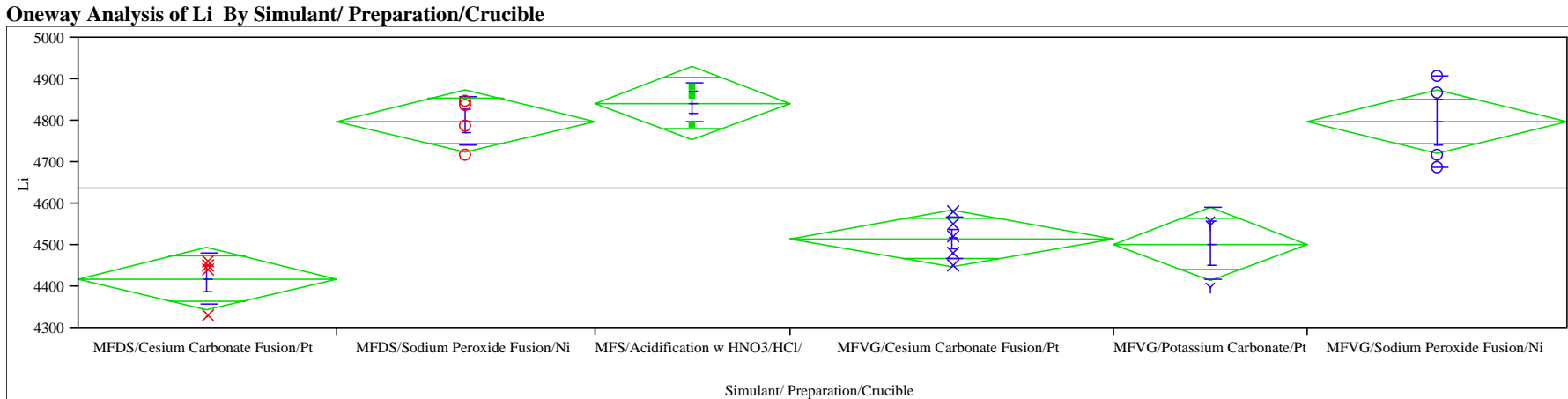

Oneway Anova

Simulant/ Preparation/Crucible

Rsquare

Adj Rsquare

Root Mean Square Erro

Mean of Response

Observations (or Sum Wgts)

Analysis of Variance

Simulant/ Preparation/Crucible

Error

C. Total

Means for Oneway Anova

Level

MFDS/Cesium Carbonate Fusion/Pt

MFS/Sodin Peroxide Fusion/Ny

MFVG/Cesimion w HNO3/HCl/

MFVG/Cesium Carbonate Fusion

MFVG/Sodium Peroxide Fusion

MFV

te of error variance

Means and Std Deviation

Level

MFDS/Cesium Carbonate Fusion/Pt

MFS/Aodum Peroxide Fusion/N/

MFVG/Cesicalon w HNO3/HCl/

MFVG Cesium Carbonate Fusion

MFVG
0.880474

0.845319

72.16335

4638.696

DF

DF
5
17
22

Sum of Squares 652132.54 88528.33

Numbe

Mean

4420.00

4800.00

4843.33

4516.00

4503.33

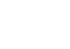

Number

Mean
4420.00

4420.00

4800.00

4843.33

4516.00

4503.33

4797.50
130427

5208

Std Error$$
\begin{aligned}
& 36.082 \\
& 36.082
\end{aligned}
$$

36.082

41.664

32.272

41.664

Std Dev

60.553

59.442

47.258

52.249

89.629
108.743
25.0457

Prob $>$ F

$\begin{array}{rr}\text { Lower 95\% } & \text { Upper 95\% } \\ 4343.9 & 4496.1 \\ 4723.9 & 4876.1 \\ 4755.4 & 4931.2 \\ 4447.9 & 4584.1 \\ 4415.4 & 4591.2 \\ 4721.4 & 4873.6\end{array}$

Std Err Mean

$$
\begin{aligned}
& 30.277 \\
& 29.721 \\
& 27.285
\end{aligned}
$$

29.721
27.285

23.367

51.747
54.371

Lower $95 \%$
4323.6
4705.4
4725.9
4451.1
4280.7
4624.5

Upper 95\%

4516.4
4894.6

4894.6

4960.7

4580.9

4726.0
4970.5 
WSRC-TR-2005-00166

SRNL-RPP-2005-00029

Revision 0

Exhibit A2. Statistical Comparisons of Preparation/Crucible Measurements by Element by Type of Sample

Oneway Analysis of Mg By Simulant/Preparation/Crucible

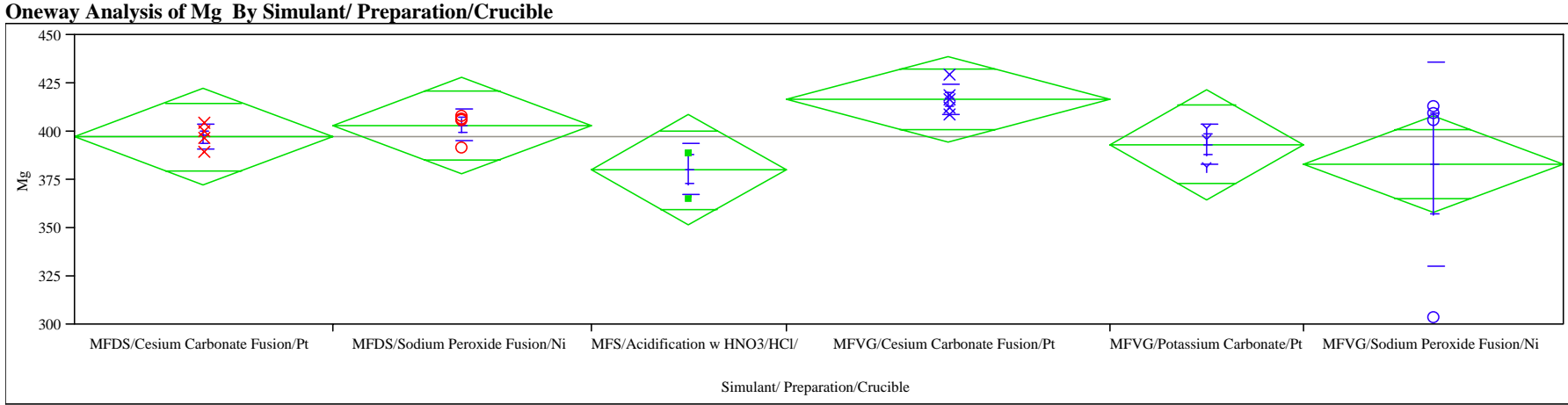

Oneway Anova

Summary of Fit

Rsquare

Adj Rsquare

Root Mean Square Erro

Mean of Response

Observations (or Sum Wgts)

Analysis of Variance

Simulant/ Preparation/Crucible

Error

Means for Oneway Anova

Means

Level

MFDS/Cesium Carbonate Fusion/Pt

MFS/Sodum Peroxide Fusion/Ny

MFVG/Cenion w HN/

MFVG/Cesium Carbonate Fusion/Pt

MFVG/Sodium Perobonate/Pt

MFVG/Sodiun Peroxide Fusion/Ni

Merror variance

Means and Std Deviations

Level

MFDS/Cesium Carbonate Fusion/Pt

MFS/Aodiam Peroxide Fusion/N/

MFVG/Cesicion w

MFV/Cesiun Carbonate Fusion/Pt

MFVG/Sodsum Peroide Fusio/

Simulant/ Preparation/Crucible

\begin{tabular}{|c|c|c|c|c|}
\hline $\begin{array}{r}0.280401 \\
0.068754 \\
23.64789 \\
397.3478 \\
23\end{array}$ & & & & \\
\hline DF & Sum of Squares & Mean Square & F Ratio & Prob $>$ F \\
\hline 5 & 3704.434 & 740.887 & 1.3249 & 0.3006 \\
\hline 17 & 9506.783 & 559.223 & & \\
\hline 22 & 13211.217 & & & \\
\hline Number & Mean & Std Error & Lower 95\% & Upper 95\% \\
\hline 4 & 397.250 & 11.824 & 372.30 & 422.20 \\
\hline 4 & 403.250 & 11.824 & 378.30 & 428.20 \\
\hline 3 & 380.333 & 13.653 & 351.53 & 409.14 \\
\hline 5 & 416.600 & 10.576 & 394.29 & 438.91 \\
\hline 3 & 393.333 & 13.653 & 364.53 & 422.14 \\
\hline 4 & 383.250 & 11.824 & 358.30 & 408.20 \\
\hline Number & Mean & Std Dev & Std Err Mean & Lower 95\% \\
\hline 4 & 397.250 & 6.3966 & 3.198 & 387.07 \\
\hline 4 & 403.250 & 7.5443 & 3.772 & 391.25 \\
\hline 3 & 380.333 & 13.2791 & 7.667 & 347.35 \\
\hline 5 & $\begin{array}{l}500.030 \\
416.600\end{array}$ & $\begin{array}{r}1.271 \\
7.9246\end{array}$ & 3.544 & 406.76 \\
\hline 3 & 393.333 & 10.2632 & 5.925 & 367.84 \\
\hline 4 & 383.250 & 52.9111 & 26.456 & 299.06 \\
\hline
\end{tabular}

Upper 95\%

407.43

415.25

413.32
426.44

418.83 
WSRC-TR-2005-00166

SRNL-RPP-2005-00029

Revision 0

Exhibit A2. Statistical Comparisons of Preparation/Crucible Measurements by Element by Type of Sample

Oneway Analysis of Mn By Simulant/Preparation/Crucible

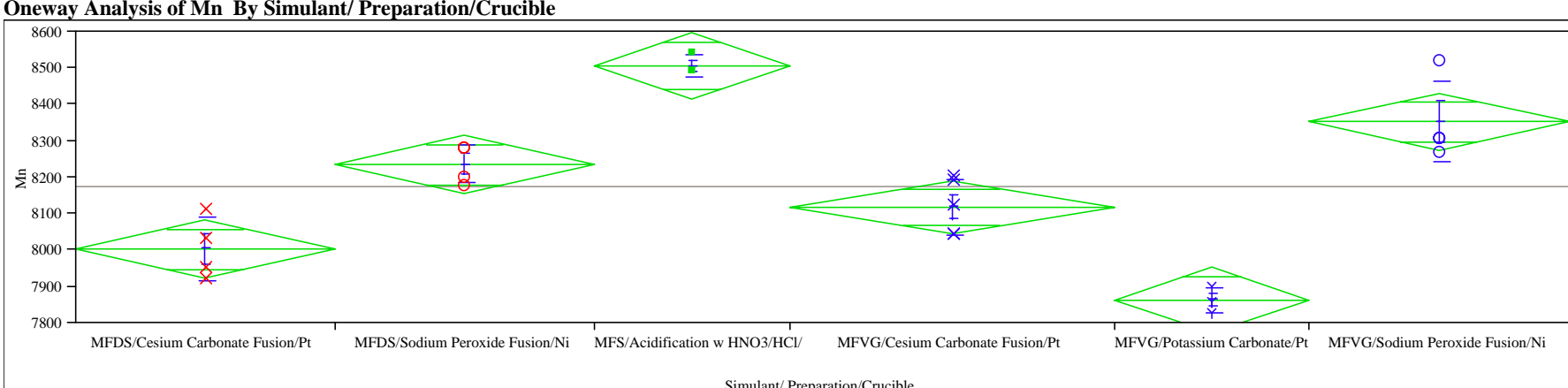

Simulant/Preparation/Crucible

Oneway Anova

Summary of Fit

Rsquare

Adj Rsquare

Root Mean Square Erro

Mean of Response

Observations (or Sum Wgts)

Analysis of Variance

Simulant/ Preparation/Crucible

Error

Means for Oneway Anova

Means

Level

MFDS/Cesium Carbonate Fusion/Pt

MFS/Sodin Peroxide Fusion/Ny

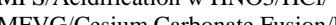

MFVG Cesium Carbonate Fusion

MFVG/Sodium Peroxide Fusion

Ste



Means and Std Deviation

Level

MFDS/Cesium Carbonate Fusion/Pt

MFS/Sodum Peroxide Fusion/N/

MFVG/Cision wNOB/HCI

MFVG/Patus Carbonate Fusion

MFVG/Sodsim Peroide Fusion

0.873881

75.48406

23

DF

DF
5
17
22

Sum of Squares

897058.41

96863.33

Number

Mean

8002.50

8235.00

8506.67

8118.00

8352.50

(1)

Number

Mean
8002.50

8002.50
8235.00
8506.67

8118.00

8118.00

7863.33
8352.50
179412
5698

Std Error$$
\begin{aligned}
& 37.742 \\
& 37.742
\end{aligned}
$$

43.581

33.757

33.742

Std Dev

85.391

52.599

28.868

35.119

77.119
113.248

$$
\begin{array}{r}
\text { F Ratio } \\
31.4876
\end{array}
$$

Prob $>$ F

$\begin{array}{rr}\text { Lower 95\% } & \text { Upper } 95 \% \\ 7922.9 & 8082.1 \\ 8155.4 & 8314.6 \\ 8414.7 & 8598.6 \\ 8046.8 & 8189.2 \\ 7771.4 & 7955.3 \\ 8272.9 & 8432.1\end{array}$

Std Err Mean

42.696

26.300
16.667

16.667

34.699

20.276
56.624
Lower 95\%

7866.6

8151.3

8435.0

8021.7

8172.3
Upper 95\% 8138.4 8318.7 8578.4
8214.3
7950.6 7950.6 
WSRC-TR-2005-00166

SRNL-RPP-2005-00029

Revision 0

Exhibit A2. Statistical Comparisons of Preparation/Crucible Measurements by Element by Type of Sample

Oneway Analysis of Mo By Simulant/ Preparation/Crucible

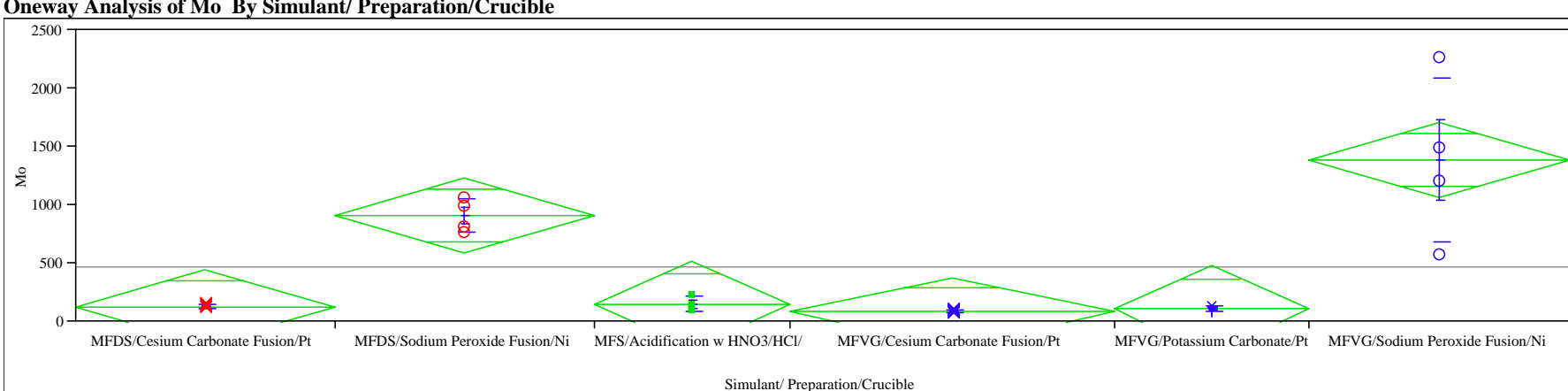

Simulant/ Preparation/Crucible

Oneway Anova

Summary of Fit

Rsquare

Adj Rsquare

Root Mean Square Erro

Mean of Response

Observations (or Sum Wgts)

Analysis of Variance

Simulant/ Preparation/Crucible

Error

Means for Oneway Anova

Means

Level

MFDS/Cesium Carbonate Fusion/Pt

MFS/Aodidin Peroxide Fusion/Mi

MFVG/Cesiation w HNO3/HCl/

MFVG/Cesium Carbonate Fusion/Pt

MFVG/Sodium Peroxide Fusio

MFVG/Soditu Peroxide Fusion/Ni



Means and Std Deviations

Level

MFDS/Cesium Carbonate Fusion/Pt

MFS/Aodum Peroxide Fusion/N/

MFVG/C i

MFVG/Cesiun Catbonate Fusion

MFVG/Sodium Peroxide Fusion/N

$\begin{array}{rrrr}\text { Sum of Squares } & \text { Mean Square } & \text { F Ratio } & \text { Prob }>F \\ 6042959.1 & 1208592 & 13.2584 & <.0001 \\ 1549659.6 & 91156 & & \\ 7592618.7 & & & \\ & & & \\ \text { Mean } & \text { Std Error } & \text { Lower 95\% } & \text { Upper 95\% } \\ 130.38 & 150.96 & -188 & 448.9 \\ 910.75 & 150.96 & 592 & 1229.2 \\ 150.00 & 174.31 & -218 & 517.8 \\ 85.10 & 135.02 & -200 & 370.0 \\ 108.67 & 174.31 & -259 & 476.4 \\ 1387.25 & 150.96 & 1069 & 1705.7 \\ & & & \\ \text { Mean } & \text { Std Dev } & \text { Std Err Mean } & \text { Lower 95\% } \\ 130.38 & 13.294 & 6.65 & 109.2 \\ 910.75 & 146.582 & 73.29 & 677.5 \\ 150.00 & 65.871 & 38.03 & -13.6 \\ 85.10 & 11.166 & 4.99 & 71.2 \\ 108.67 & 22.396 & 12.93 & 53.0 \\ 1387.25 & 701.068 & 350.53 & 271.7\end{array}$


WSRC-TR-2005-00166

SRNL-RPP-2005-00029

Revision 0

Exhibit A2. Statistical Comparisons of Preparation/Crucible Measurements by Element by Type of Sample

Oneway Analysis of Na By Simulant/Preparation/Crucible

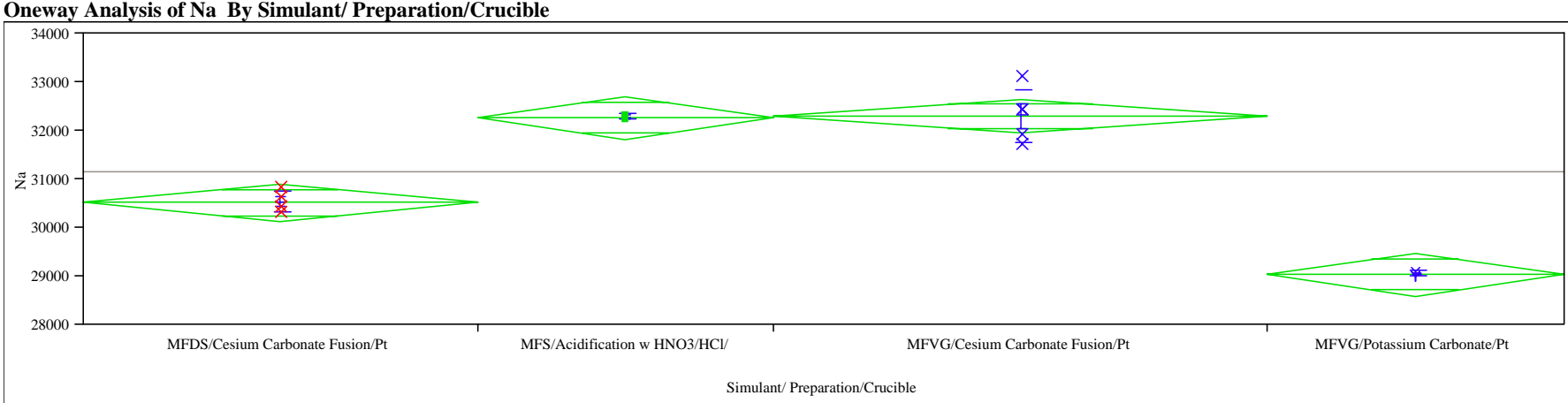

Missing Rows

Anova

Summary of Fit

Rsquare

Adj Rsquare

Root Mean Square

Mean of Response

Analysis of Variance

Source

Simulant/ Preparation/Crucible

Error

C. Total

Means for Oneway Anova

Level

MFDS/Cesium Carbonate Fusion/Pt

MFS/Acidification w HNO3/HCl/

MFVG Cosium Carbonate Fusion/Pt

MFVG/Potassium Carbonate/Pt

Std Erior usses a pooled estimate of error variance

Means and Std Deviations

MFDS/Cesium Carbonate Fusion/P

MFS/Acidification w HNO3/HCl/

MFVG Potassiur Canate Fusion

\begin{tabular}{|c|c|c|c|c|}
\hline $\begin{array}{r}0.949769 \\
0.93607 \\
349.1331 \\
31166.67 \\
15\end{array}$ & & & & \\
\hline DF & Sum of Squares & Mean Square & F Ratio & Prob $>$ F \\
\hline 3 & 25352500 & 8450833 & 69.3294 & $<.0001$ \\
\hline 11 & 1340833 & 121894 & & \\
\hline 14 & 26693333 & & & \\
\hline Number & Mean & Std Error & Lower 95\% & Upper 95\% \\
\hline 4 & 30525.0 & 174.57 & 30141 & 30909 \\
\hline 3 & 32266.7 & 201.57 & 31823 & 32710 \\
\hline 5 & 32300.0 & 156.14 & 31956 & 32644 \\
\hline 3 & 29033.3 & 201.57 & 28590 & 29477 \\
\hline Number & Mean & Std Dev & Std Err Mean & Lower 95\% \\
\hline 4 & 30525.0 & 221.736 & 110.87 & 30172 \\
\hline 3 & 32266.7 & 57.735 & 33.33 & 32123 \\
\hline 5 & 32300.0 & 543.139 & 242.90 & 31626 \\
\hline 3 & 29033.3 & 57.735 & 33.33 & 28890 \\
\hline
\end{tabular}

Upper 95\%

30878

32410

29177 
WSRC-TR-2005-00166

SRNL-RPP-2005-00029

Revision 0

Exhibit A2. Statistical Comparisons of Preparation/Crucible Measurements by Element by Type of Sample

Oneway Analysis of Ni By Simulant/ Preparation/Crucible

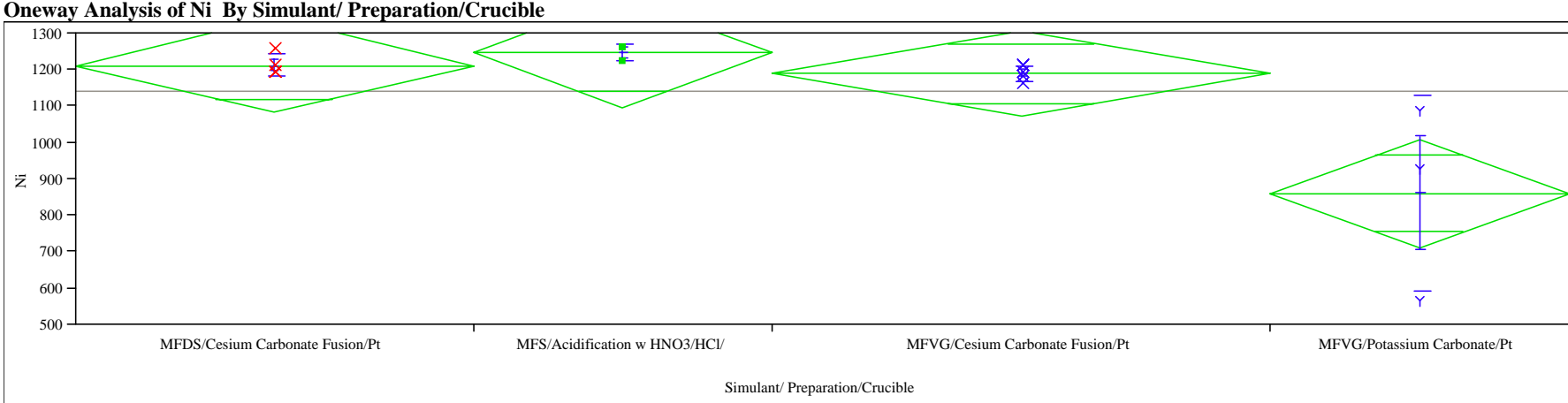

Missing Rows

(a)

Rsquare

Adj Rsquare

Root Mean Square Erro

Mean of Response

Observations (or Sum Wgts)

Analysis of Variance

Simulant/ Preparation/Crucible

C. Total

Means for Oneway Anova

Level

MFDS/Cesium Carbonate Fusion/Pt

MFS/Acidification w HNO3/HCl/

MFVG/Csium Cabonate Fusion

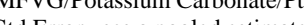

Std Erior uses a poold estimate of error variance

Means and Std Deviations

Level

MFDS/Cesium Carbonate Fusion/P

MFS/Acidification w HNO3/HCl/

MFVG/Potassiun Conate Fusion

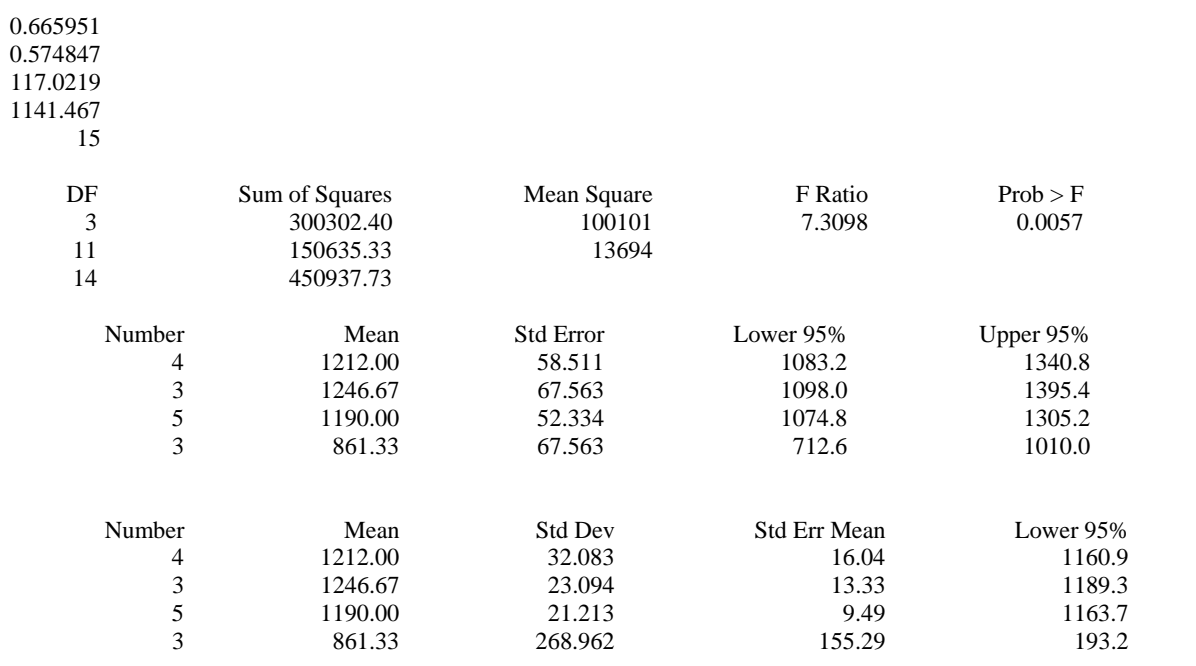

Upper $95 \%$
1263.1
1304.0
1216.3
1529.5 
WSRC-TR-2005-00166

SRNL-RPP-2005-00029

Revision 0

Exhibit A2. Statistical Comparisons of Preparation/Crucible Measurements by Element by Type of Sample

Oneway Analysis of P By Simulant/Preparation/Crucible

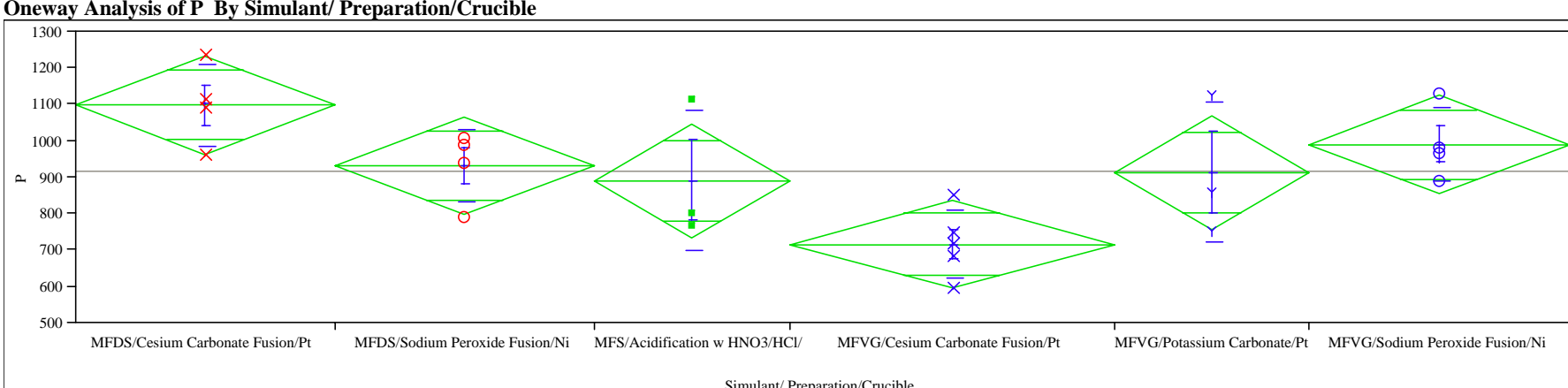

Simulant/Preparation/Crucible

Oneway Anova

Summary of Fit

Rsquare

Adj Rsquare

Root Mean Square Erro

Mean of Response

Observations (or Sum Wgts)

Analysis of Variance

Simulant/ Preparation/Crucible

Error

Means for Oneway Anova

Means

Level

MFDS/Cesium Carbonate Fusion/Pt

MFS/Sodum Peroxide Fusion/Ny

MFVG/C i Caton wNOB/HCI

MFVG/Cesium Carbonate Fusion/Pt

MFVG/Sodium Peroxonate/Pt

MFVG/Soditum Peroxide Fusion/Ni

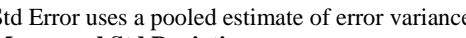

Means and Std Deviations

Level

MFDS/Cesium Carbonate Fusion/Pt

MFS/Aodian Peroxide Fusion/N/

FFVG/Cesicion w

MFVG/Cesiun Carbonate Fusion/Pt

MFVG/Solim Peroxide Fusior

\begin{tabular}{|c|c|c|c|c|c|}
\hline $\begin{array}{r}0.562657 \\
0.434027 \\
127.781 \\
916.9565 \\
23\end{array}$ & & & & & \\
\hline DF & Sum of Squares & Mean Square & F Ratio & Prob $>$ F & \\
\hline 5 & 357111.12 & 71422.2 & 4.3742 & 0.0096 & \\
\hline 17 & 277575.83 & 16328.0 & & & \\
\hline 22 & 634686.96 & & & & \\
\hline Number & Mean & Std Error & Lower 95\% & Upper 95\% & \\
\hline 4 & 1098.75 & 63.891 & 963.95 & 1233.5 & \\
\hline 4 & 932.50 & 63.891 & 797.70 & 1067.3 & \\
\hline 3 & 891.67 & 73.774 & 736.02 & 1047.3 & \\
\hline 5 & 717.00 & 57.145 & 596.43 & 837.6 & \\
\hline 3 & 913.33 & 73.774 & 757.68 & 1069.0 & \\
\hline 4 & 991.25 & 63.891 & 856.45 & 1126.0 & \\
\hline Number & Mean & Std Dev & Std Err Mean & Lower 95\% & Upper 95\% \\
\hline 4 & 1098.75 & 112.574 & 56.29 & 919.62 & 1277.9 \\
\hline 4 & 932.50 & 99.457 & 49.73 & 774.24 & 1090.8 \\
\hline 3 & 891.67 & 189.890 & 109.63 & 419.95 & 1363.4 \\
\hline 5 & 717.00 & 93.180 & 41.67 & 601.30 & 832.7 \\
\hline 3 & 913.33 & 190.679 & 110.09 & 439.66 & 1387.0 \\
\hline 4 & 991.25 & 100.530 & 50.26 & 831.28 & 1151.2 \\
\hline
\end{tabular}


WSRC-TR-2005-00166

SRNL-RPP-2005-00029

Revision 0

Exhibit A2. Statistical Comparisons of Preparation/Crucible Measurements by Element by Type of Sample

Oneway Analysis of Pb By Simulant/Preparation/Crucible

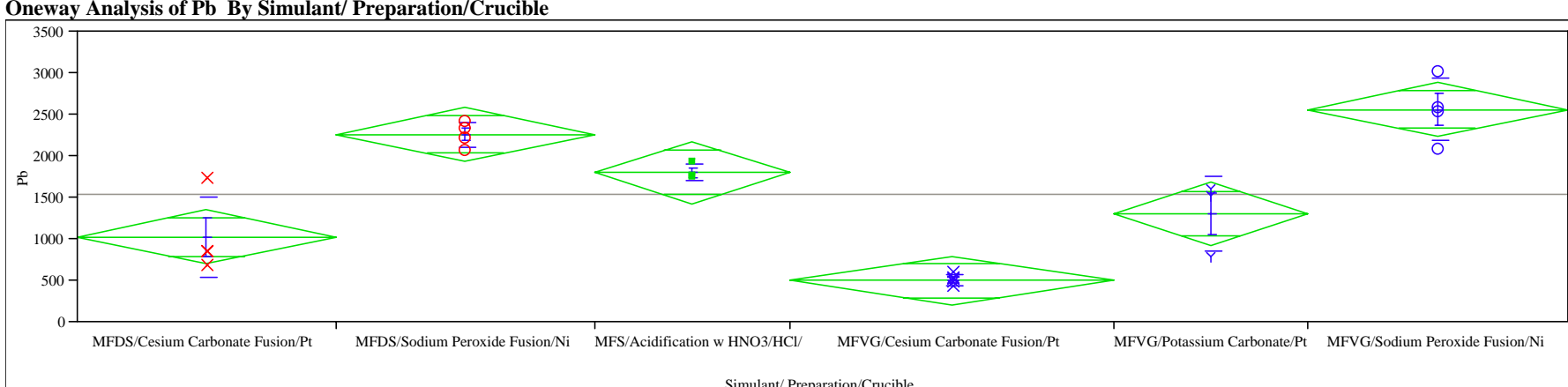

Simulant/ Preparation/Crucible

Oneway Anova

Summary of Fit

Rsquare

Adj Rsquare

Root Mean Square Erro

Mean of Response

Observations (or Sum Wgts)

Analysis of Variance

Simulant/ Preparation/Crucible

Error

Means for Oneway Anova

Means

Level

MFDS/Cesium Carbonate Fusion/Pt

MFS/Sodin Peroxide Fusion/Ny

MFVG/Cesimion w HNO3/HCl/

MFVG/Cesium Carbonate Fusion

MFVG/Sodium Peroxide Fusion

MFVG/Sodlun Peroxide Fusion/Ni



Means and Std Deviations

Level

MFDS/Cesium Carbonate Fusion/Pt

MFS/Sodum Peroxide Fusion/N/

MFVG/C i C WNOB/HCl/

MFVG/Cesiun Carbonate Fusion/P

MFVG/Solium Peroxide Fusior

0.889848
0.85745
308.6556
1534.717
23
$\mathrm{DF}$
5
17
22
$\mathrm{~N}$

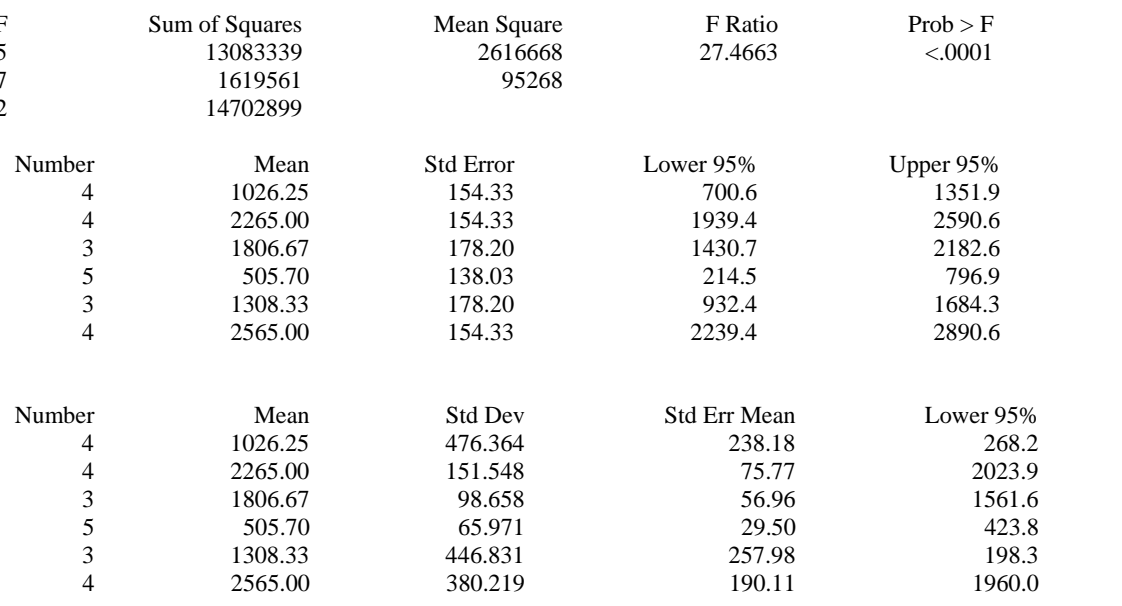

Upper $95 \%$
1784.3
2506.1
2051.7
587.6
2418.3
3170.0 
WSRC-TR-2005-00166

SRNL-RPP-2005-00029

Revision 0

Exhibit A2. Statistical Comparisons of Preparation/Crucible Measurements by Element by Type of Sample

Oneway Analysis of S By Simulant/Preparation/Crucible

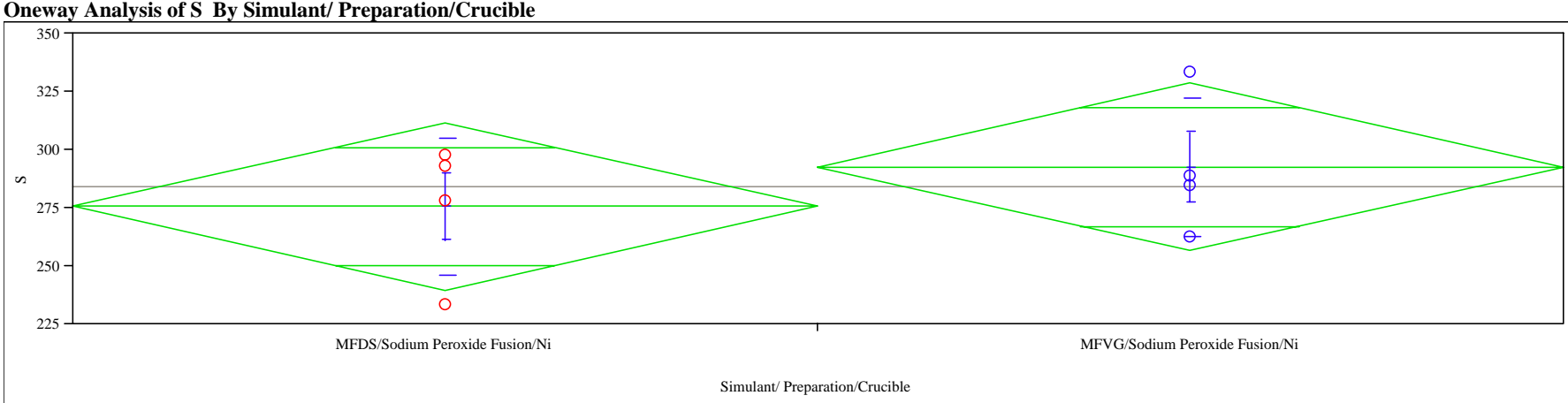

Missing Rows

15Oneway Anov

ummary of Fit

Rsquare

Adj Rsquare

Root Mean Square Error

Mean of Response

0.09594

(29.050

Observations (or Sum Wgts)

Preparation/Crucible

MFDS/Sodium Peroxide Fusion/Ni-MFVG/Sodium Peroxide Fusionsi

Assuming equal variances

Difference

Std Err Dif

Upper CL Dif

Lower CL Dif

-17.000 t Ratio

20.821 DF

33.948 Prob $>|t|$

0.95 Prob $<\mathrm{t}$

$-0.81647$

6
0.4454
0.7773

0.4454
0.7773

Analysis of Variance

Analysis o

Source
Simulant/ Preparation/Crucible

Error

C. Total

Means

MFDS/Sodium Peroxide Fusion/N

MFVG/Sodium Peroxide Fusion/Ni

Std Error uses a pooled estimate of error variance

Means and Std Deviations

Level

MFDS/Sodium Peroxide Fusion/Ni

MFVG/Sodium Peroxide Fusion/Ni

Sum of Squares

578.0000

5202.3750

Mean

Number

Mean
275.625
292.625

Number

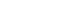

Mean
275.625
292.625
Mean Square

578.000

867.063

Std Error

14.723

Std Dev

29.3410
29.5505
239.60

Std Err Mean

14.671
14.775

Upper 95\% 322.31 
WSRC-TR-2005-00166

SRNL-RPP-2005-00029

Revision 0

Exhibit A2. Statistical Comparisons of Preparation/Crucible Measurements by Element by Type of Sample

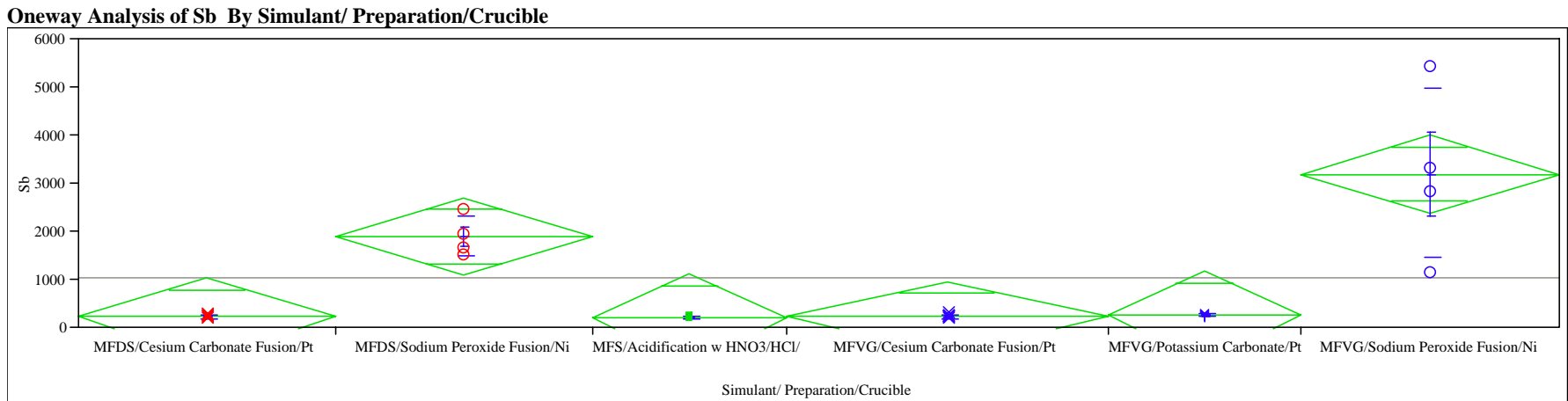

Oneway Anova

ummary of Fit

Rsquare

Adj Rsquare

Root Mean Square Erro

Mean of Response

Observations (or Sum Wgts)

Analysis of

Source

Simulant/ Preparation/Crucible

Error

C. Total

Means

Level

MFDS/Cesium Carbonate Fusion/Pt

列

MFVG/Cesium Carbonate Fusion/Pt

MFVG/Potassium Carbonate/Pt

MFVG/Sodium Peroxide Fusion/Ni

Std Error uses a pooled estimate of error variance

Means and Std Deviations

Level

MFDS/Cesium Carbonate Fusion/Pt

MFDS/Sodium Peroxide Fusion/N

MFS/Acidification w HNO3/HCl

MFVG/Cesium Carbonate Fusion/Pt

MFVG/Potassium Carbonate/Pt

MFVG/Sodium Peroxide Fusion/Ni
0.760769
0.690407
761.4143
1040.522

DF

5
17
22

Sum of Squares

31341941
9855779

9855779

Number

Mean

Mean
229.50
1905.00

212.33
233.60

266.33

3197.50

Number

Mean
229.50
1905.00
212.33
233.60
266.33
3197.50

Mean
229.50
1905.00
212.33
233.60
266.33
3197.50

3250

32.50

414.13
19.50
38.41

38.41

28.04
1763.51

Std Error
380.71
380.71
439.60
340.51
439.60
380.71


Std Dev
32.50
414.13
19.50
38.41
28.04
1763.51

-574
1102
-715

-715
-485
-661
2394

F Ratio

Prob $>$ F

6268388

10.8122

$<.0001$

Std Err Mean

16.25
207.06
11.26

11.26
17.18

17.18

16.19
881.75
Upper 95\%

1032.7

2708.2

1139.8

952.0

1193.8

Lower 95\%

177.8

1246.0
163.9

163.9
185.9
196.7

196.7
Upper 95\% 281.2
2564.0 2564.0
260.8 260.8
281.3
336.0

336.0
6003.6 
WSRC-TR-2005-00166

SRNL-RPP-2005-00029

Revision 0

Exhibit A2. Statistical Comparisons of Preparation/Crucible Measurements by Element by Type of Sample

Oneway Analysis of Si By Simulant/ Preparation/Crucible

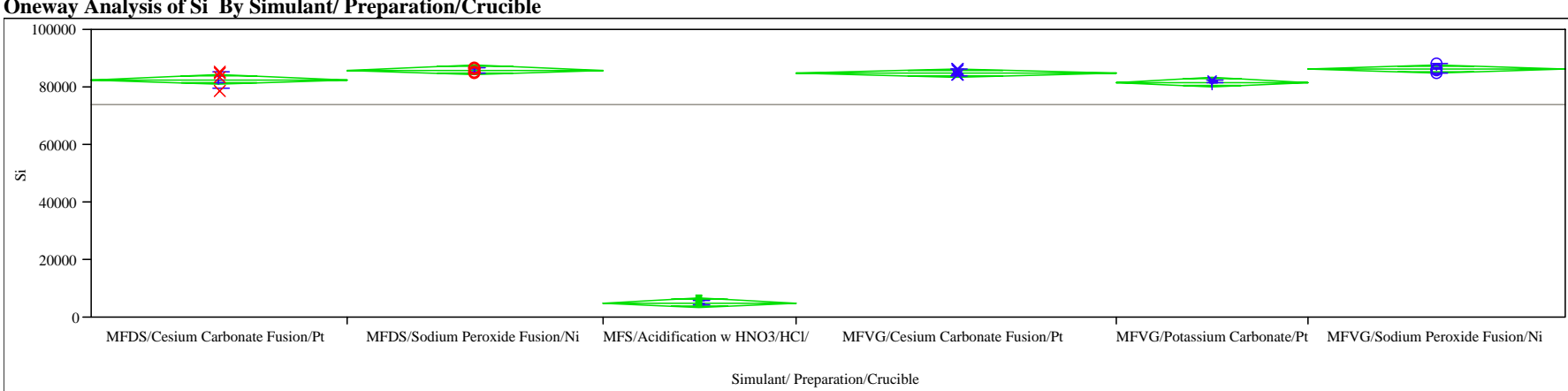

Oneway Anova

Rsquare

Adj Rsquare

Root Mean Square Error

Mean of Response

Observations (or Sum Wgts)

Analysis of Variance

Simulant/Preparation/Crucible

C. Total

Means for Oneway Anova

Level

MFDS/Cesium Carbonate Fusion/Pt

MFDS/Sodium Peroxide Fusion/Ni

MFS/Acidification w $\mathrm{HNO} / \mathrm{HCl}$

MFVG/Cesium Carbonate Fusion/Pt

MFVG/Potassium Carbonate/Pt

MFVG/Sodium Peroxide Fusion/Ni

Std Error uses a pooled estimate of error variance

Means and Std Deviations

Level

MFDS/Cesium Carbonate Fusion/Pt

MFDS/Sodium Peroxide Fusion/Ni

MFS/Acidification w $\mathrm{HNO} / \mathrm{HCl} /$

MFVG/Cesium Carbonate Fusion/P

MFVG/Potassium Carbonate/Pt

MFVG/Sodium Peroxide Fusion/Ni

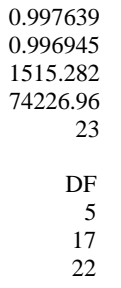

0.997639

0.996945

1515.282

.96

DF
5
17
22

Sum of Squares

$1.6494 \mathrm{e}+10$
39033366.7

$1.65331 \mathrm{e} 10$

$22 \quad 1.65331 \mathrm{e} 10$

Numbe

Mean
82725.0

82725.0
86025.0
5206.7

5206.7

85000.0

86475.0

Mean
82725.0

82725.0
86025.0

5206.7
85000.0

85000.0

81900.0
Mean Square
$3.2988 \mathrm{e}^{+9}$
2296080.4
Std Error
757.64
757.64
874.85
874.85
877.65
874.85
874.85
757.64
Std Dev
853.91
853.91
908.53
556.78
1314.98

F Ratio

Prob $>$ F

$\begin{array}{rr}\text { Lower } 95 \% & \text { Upper } 95 \% \\ 81127 & 84323 \\ 84427 & 87623 \\ 3361 & 7052 \\ 83570 & 86430 \\ 80054 & 83746 \\ 84877 & 88073\end{array}$

Std Err Mean

1448.8
427.0
524.5
458.3
321.5
657.5

78114

78114
84666

84666
2950

2950
83728

83728
80517

84383
Upper $95 \%$
87336
87384
7464
86272
83283
88567 
WSRC-TR-2005-00166

SRNL-RPP-2005-00029

Revision 0

Exhibit A2. Statistical Comparisons of Preparation/Crucible Measurements by Element by Type of Sample

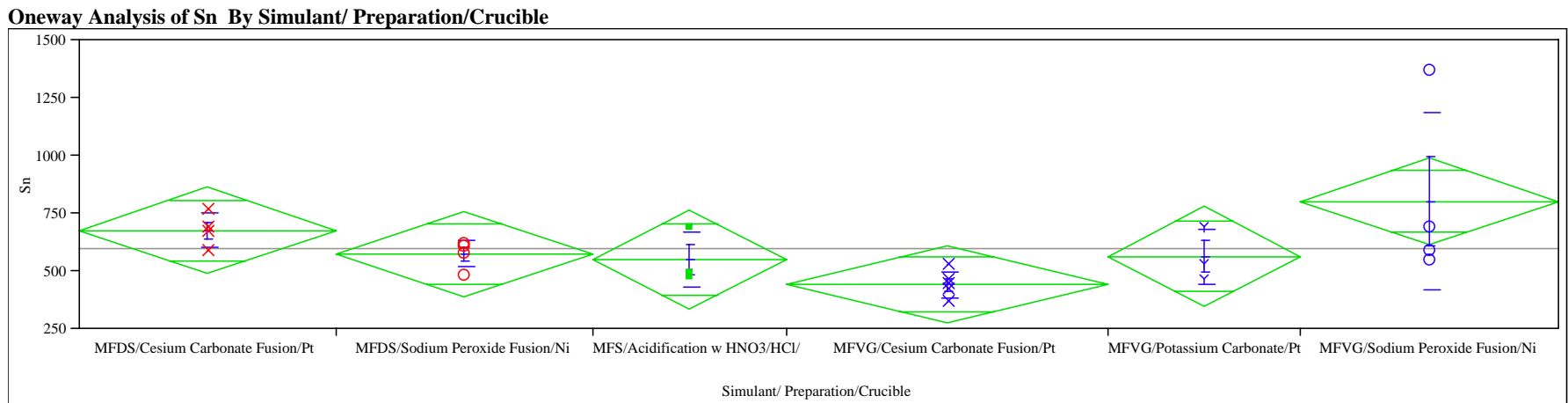

Oneway Anova

ummary of Fit

Rsquare

Adj Rsquare

Root Mean Square Erro

Mean of Response

Observations (or Sum Wgts)

Analysis of

Source

Simulant/ Preparation/Crucible

Error

C. Total

Means

Level

MFDS/Cesium Carbonate Fusion/Pt

Sodium Peroxide Fusion/ $\mathrm{N}$

$\mathrm{MFS} /$ Acidification w $\mathrm{HNO} / \mathrm{HCl}$

MFVG/Cesium Carbonate Fusion/Pt

MFVG/Potassium Carbonate/Pt

MFVG/Sodium Peroxide Fusion/Ni

Std Error uses a pooled estimate of error variance

Means and Std Deviations

Level

MFDS/Cesium Carbonate Fusion/Pt

MFDS/Sodium Peroxide Fusion/Ni

MFS/Acidification w HNO3/HCl

MFVG/Cesium Carbonate Fusion/Pt

MFVG/Potassium Carbonate/Pt

MFVG/Sodium Peroxide Fusion/Ni
0.377872

0.194894

177.612

23

DF
5
17
22

Sum of Squares

325734.43

53628.07

Number

$$
\text { Mean }
$$

Mean
676.250
574.375

574.375
550.167

550.167
442.200

442.200
563.833

802.500

Number

Mean
676.250
574.375
550.167
442.200
563.833
802.500

Mean
676.250
574.375
550.167
442.200
563.833
802.500

$$
\begin{array}{r}
73.754 \\
60.359 \\
117.218 \\
57.687 \\
117.947 \\
383.156
\end{array}
$$$$
\begin{array}{r}
\text { Std Dev } \\
73.754 \\
60.359
\end{array}
$$

Mean Square
65146.9
31546.


td Error
88.81
88.81
102.54
79.43
102.54
88.81


Std Dev
73.754
60.359
117.218
57.687
117.947
383.156

$2.0651 \quad$ Prob $>$ F

3146.9

$\begin{array}{rr}\text { Lower 95\% } & \text { Upper 95\% } \\ 488.88 & 863.62 \\ 387.01 & 761.74 \\ 333.82 & 766.52 \\ 274.62 & 609.78 \\ 347.48 & 780.18 \\ 615.13 & 989.87\end{array}$

Std Err Mean

36.88
30.18
67.68

30.18
67.68
2580

25.80

68.10
191.58
Lower 95\%
558.89
478.33
258.98
370.57
192.81

Upper 95\%

793.6

670.4

841.4
513.8

513.8
856.8

856.8
1412.2 
WSRC-TR-2005-00166

SRNL-RPP-2005-00029

Revision 0

Exhibit A2. Statistical Comparisons of Preparation/Crucible Measurements by Element by Type of Sample

Oneway Analysis of Sr By Simulant/ Preparation/Crucible

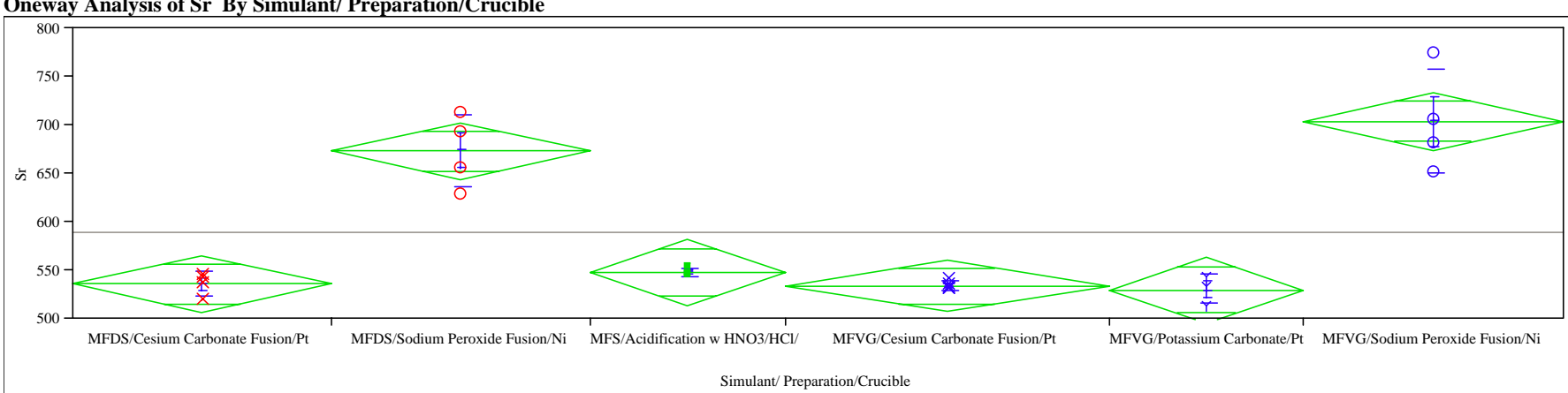

Oneway Anova

Simulant/Preparation/Crucible

Summary of Fit

Rsquare

Adj Rsquare

Root Mean Square Erro

Mean of Response

Observations (or Sum Wgts)

Analysis of Variance

Simulant/ Preparation/Crucible

Error

Means for Oneway Anova

Means

Level

MFDS/Cesium Carbonate Fusion/Pt

MES/Acdific Pexoxide Fusion/Ni/

MFVG/Cesim Wa whel/

MFVG Cesilun Cabonase Fusir

MFVG/Sodiun Cabonast

MFVG/Possim Cabion

error variance

Leve

FDS/Cesium Carbonate Fusion/Pt

oxide Fusion/N

列

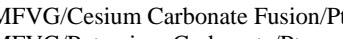

MFVG/Sodiun CabonatPt

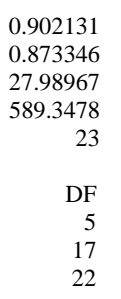

0.902131

0.873346

27.98967

23

DF
5
17
22

Sum of Squares 122763.05

13318.17

Number

Mean

535.750

673.000

548.333

534.000

703.750

Mean
535.750

673.000

548.333

534.000

703.750
13.995
16.995
16.160
16.517
16.160
Std Dev
11.6440
37.1394
4.1633
4.2426
14.7309
14.7309

Mean Square

24552.6

F Ratio

Prob $>$ F

$\begin{array}{rr}\text { Number } & \text { Mean } \\ 4 & 535.750 \\ 4 & 673.000 \\ 3 & 548.333 \\ 5 & 534.000 \\ 3 & 530.000 \\ 4 & 703.750\end{array}$

$\begin{array}{rr}\text { Lower } 95 \% & \text { Upper } 95 \% \\ 506.22 & 565.28 \\ 643.47 & 702.53 \\ 514.24 & 582.43 \\ 507.59 & 560.41 \\ 495.91 & 564.09 \\ 674.22 & 733.28\end{array}$

Std Err Mean

5.822
18.570

18.570
2.404
1.897

1.897
8505

8.505
26.193

Lower $95 \%$
517.22
613.90
537.99
528.73
493.41
620.39

Upper 95\%

554.28

732.10

558.68
539.27
566.59

566.59 
WSRC-TR-2005-00166

SRNL-RPP-2005-00029

Revision 0

Exhibit A2. Statistical Comparisons of Preparation/Crucible Measurements by Element by Type of Sample

Oneway Analysis of Th By Simulant/Preparation/Crucible

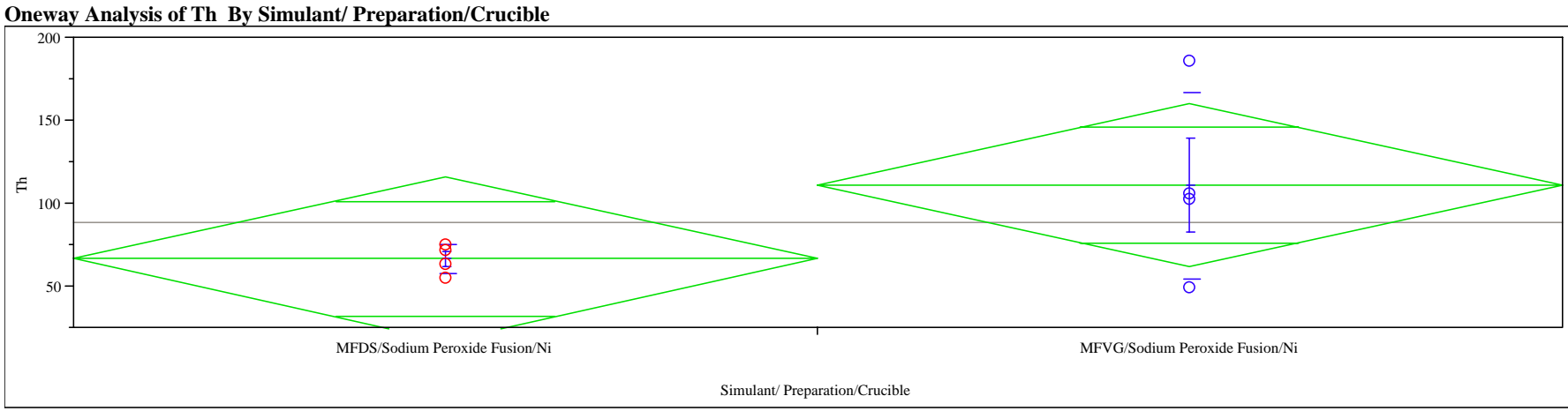

Missing Rows

15Oneway Anova

ummary of Fit

Rsquare

Adj Rsquare

Root Mean Square Error

Mean of Respons

0.288733

0.17018

88.975

Observations (or Sum Wgts)

MFDS/Sodium Peroxide Fusion/Ni-MFVG/Sodium Peroxide Fusion/N

Assuming equal variances

Difference

Std Err Dif

Upper CL Dif

Lower CL Dif

-44.40 t Ratio

25.21 Prob >

114.01 Prob $>t$

Analysis of Variance

Analysis o

0.95 Prob $<\mathrm{t}$

6
0.1696
0.9152
0.0848

Simulant/ Preparation/Crucible

Error

Means for Oneway Anova

Level

MFDS/Sodium Peroxide Fusion/Ni

MFVG/Sodium Peroxide Fusion/N

Std Error uses a pooled estimate of error variance

Means and Std Deviations

Level

MFDS/Sodium Peroxide Fusion/Ni

MFVG/Sodium Peroxide Fusion/Ni

\begin{tabular}{|c|c|c|c|c|c|}
\hline DF & Sum of Squares & Mean Square & F Ratio & Prob $>F$ & \\
\hline 1 & 3942.720 & 3942.72 & 2.4357 & 0.1696 & \\
\hline 6 & 9712.515 & 1618.75 & & & \\
\hline 7 & 13655.235 & & & & \\
\hline Number & Mean & Std Error & Lower 95\% & Upper 95\% & \\
\hline 4 & 66.775 & 20.117 & 17.551 & 116.00 & \\
\hline 4 & 111.175 & 20.117 & 61.951 & 160.40 & \\
\hline Number & Mean & Std Dev & Std Err Mean & Lower 95\% & Upper 95\% \\
\hline 4 & 66.775 & 8.9619 & 4.481 & 52.515 & 81.04 \\
\hline 4 & 111.175 & 56.1889 & 28.094 & 21.766 & 200.58 \\
\hline
\end{tabular}


WSRC-TR-2005-00166

SRNL-RPP-2005-00029

Revision 0

Exhibit A2. Statistical Comparisons of Preparation/Crucible Measurements by Element by Type of Sample

Oneway Analysis of Ti By Simulant/ Preparation/Crucible

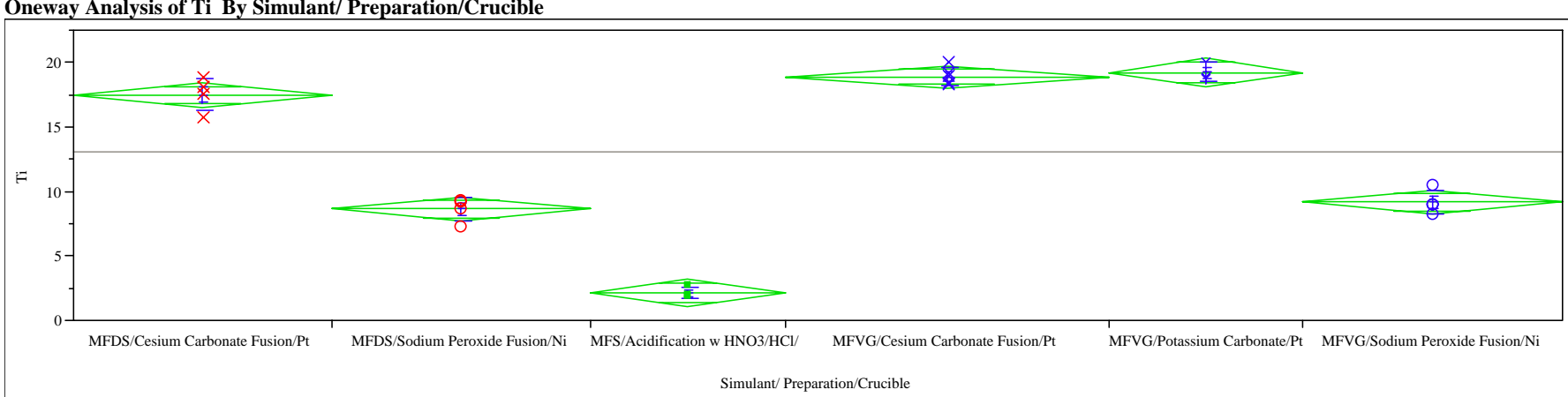

Oneway Anova

ummary of Fit

Rsquare

Root Mean Square Error

Moan of Response

Observations (or Sum Wgts)

Analysis of Variance

Simulant/ Preparation/Crucible

Error

Means for Oneway Anova

Means

Level

MFDS/Cesium Carbonate Fusion/Pt

MS/Acidum Paxide Fusion/Ni

MFVG/Cesim

MFVG/Cesium Cabonate Fusiont

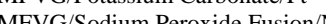

Serion $/ \mathrm{N}$

error variance

ation

Level

FDS/Cesium Carbonate Fusion/Pt

DS/Acidificalon w HNO3/HCl/

MFVG/Cesim Caw

MFVG/Posassiu Cana Tusion

MFVG/Sodium Peroxide Fusion
0.984307

0.979692

0.896821

073

DF

DF
5
17
22

Sum of Squares 857.60855 13.67291

Numbe

Mean

17.5000
8.6875

8.6875
2.1817

18.9400

18.9400
19.2667

9.2250

Number

Mean
17.5000
8.6875
2.1817
18.9400
19.2667
9.2250

Mean
17.5000
8.6875
2.1817
18.9400
19.2667
9.2250

1.31403

0.93397

0.46361

0.68044

0.72342
Mean Square

171.522
0.804

F Ratio

213.2589

Prob $>$ F

td Error

0.4484

0.44841

$\begin{array}{rr}\text { Lower 95\% } & \text { Upper 95\% } \\ 16.554 & 18.446 \\ 7.741 & 9.634 \\ 1.089 & 3.274 \\ 18.094 & 19.786 \\ 18.174 & 20.359 \\ 8.279 & 10.171\end{array}$

Std Err Mean 0.65701

0.46698

0.30430

0.41767
Upper 95\%

19.591

3.333
19.785

19.785

21.064 
WSRC-TR-2005-00166

SRNL-RPP-2005-00029

Revision 0

Exhibit A2. Statistical Comparisons of Preparation/Crucible Measurements by Element by Type of Sample

Oneway Analysis of Tl By Simulant/ Preparation/Crucible

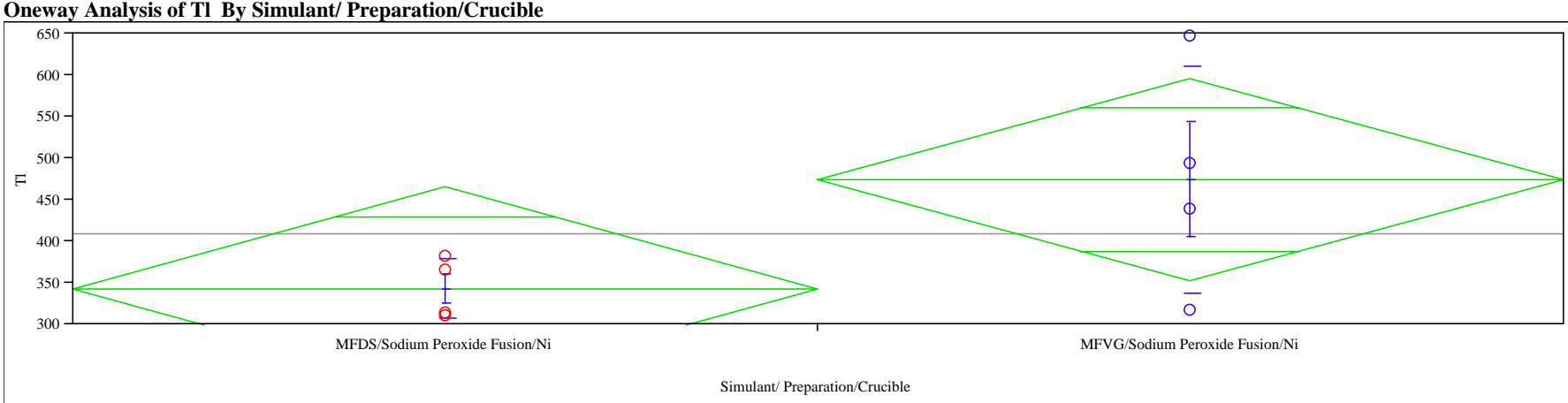

Missing Rows

15Oneway Anov

Rsquare

Adj Rsquare

0.365524

Root Mean Square Error

0.259778

Mean of Response

Observations (or Sum Wgts)

408.875

Test

Assuming equal variances

Difference

Std Err Dif

Upper CL Dif

Lower CL Dif

-131.25 t Ratio
$70.59 \mathrm{DF}$

(1)

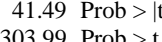

-0.95 Prob $<t$

6
0.1123
0.9438

0.1123

Analysis of Variance

Analysis o

Source

Simul

Error

C. Total

Means

MFDS/Sodium Peroxide Fusion/N

MFVG/Sodium Peroxide Fusion/N

Std Error uses a pooled estimate of error variance

Means and Std Deviations

Level

MFDS/Sodium Peroxide Fusion/Ni

MFVG/Sodium Peroxide Fusion/Ni

$\begin{array}{rrrrrr}\text { DF } & \text { Sum of Squares } & \text { Mean Square } & \text { F Ratio } & \text { Prob > F } & \\ 1 & 34453.125 & 34453.1 & 3.4566 & 0.1123 & \\ 6 & 59803.750 & 9967.3 & & & \\ 7 & 94256.875 & & & & \\ & & & & & \\ \text { Number } & \text { Mean } & \text { Std Error } & \text { Lower 95\% } & \text { Upper 95\% } \\ 4 & 343.250 & 49.918 & 221.10 & 465.40 & \\ & 474.500 & 49.918 & 352.35 & 596.65 & \\ & & & & & \text { Upper 95\% } \\ & & & & & \\ \text { Number } & \text { Mean } & \text { Std Dev } & \text { Std Err Mean } & \text { Lower 95\% } & \\ 4 & 343.250 & 36.124 & 18.062 & 285.77 & 600.73 \\ 4 & 474.500 & 136.491 & 68.245 & 257.31 & \end{array}$


WSRC-TR-2005-00166

SRNL-RPP-2005-00029

Revision 0

Exhibit A2. Statistical Comparisons of Preparation/Crucible Measurements by Element by Type of Sample

Oneway Analysis of U By Simulant/ Preparation/Crucible

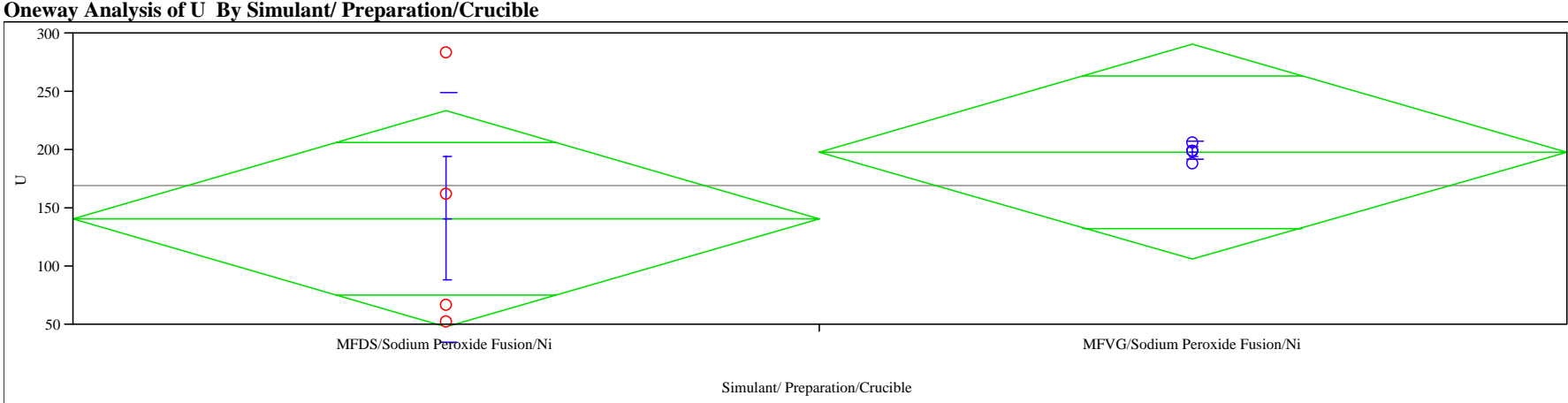

Missing Rows

15Oneway Anova

ummary of Fit

Adj Rsquare

Root Mean Square Error

Mean of Respons

0.01936

Observations (or Sum Wgts)

nt/ Preparation/Crucible

Deroxide Fusion/Ni-MFVG/Sodium Peroxide Fusion/N

Assuming equal variances

Difference

Std Err Dif

Upper CL Dif

Lower CL Dif

-57.13 t Ratio
53.54 DF

53.54 DF

-188.14 Prob $>t$

0.95 Prob $<\mathrm{t}$

$-1.06687$

6
0.3271

0.3271

0.8365

Analysis of Variance

Source

Simulant/ Preparation/Crucible

Error

C. Total

Means for Oneway Anova

Level

MFDS/Sodium Peroxide Fusion/N

MFVG/Sodium Peroxide Fusion/N

Std Error uses a pooled estimate of error variance

Means and Std Deviations

Level

MFDS/Sodium Peroxide Fusion/N

MFVG/Sodium Peroxide Fusion/Ni

\begin{tabular}{|c|c|c|c|c|c|}
\hline DF & Sum of Squares & Mean Square & F Ratio & Prob $>F$ & \\
\hline 1 & 6526.531 & 6526.53 & 1.1382 & 0.3271 & \\
\hline 6 & 34404.438 & 5734.07 & & & \\
\hline 7 & 40930.969 & & & & \\
\hline Number & Mean & Std Error & Lower 95\% & Upper 95\% & \\
\hline 4 & 141.625 & 37.862 & 48.98 & 234.27 & \\
\hline 4 & 198.750 & 37.862 & 106.11 & 291.39 & \\
\hline Number & Mean & Std Dev & Std Err Mean & Lower 95\% & Upper 95\% \\
\hline 4 & 141.625 & 106.833 & 53.416 & -28.4 & 311.62 \\
\hline 4 & 198.750 & 7.411 & 3.705 & 187.0 & 210.54 \\
\hline
\end{tabular}


WSRC-TR-2005-00166

SRNL-RPP-2005-00029

Revision 0

Exhibit A2. Statistical Comparisons of Preparation/Crucible Measurements by Element by Type of Sample

Oneway Analysis of Y By Simulant/ Preparation/Crucible

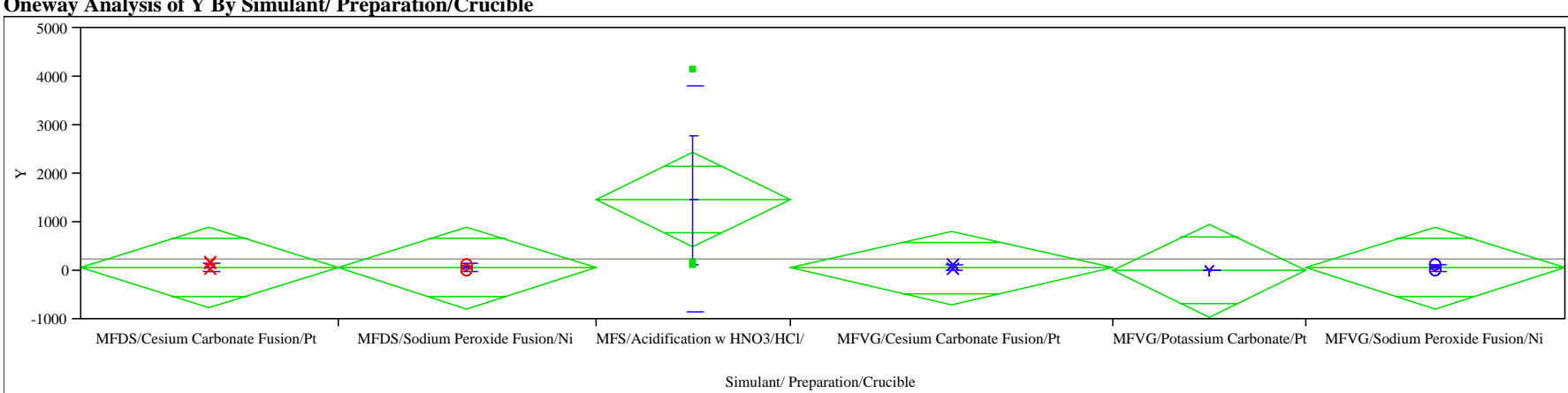

Oneway Anova

Simulant/ Preparation/Crucible

ummary of Fit

Rsquare

Adj Rsquare

Root Mean Square Error

Mean of Response

Observations (or Sum Wgts)

Analysis of Variance

Source

Simulant/ Preparation/Crucible

Error

Means for Oneway Anova

Level

MFDS/Cesium Carbonate Fusion/Pt

MFS/Sodum Peroxide Fusion/Ny

MFVG/Csiun w HO3/HCl/

MFVG/Cesium Carbonate Fusion/Pt

MFVG/Sodium Pexponate/

MFV

of error variance

Means and Std Deviations

Level

MFDS/Cesium Carbonate Fusion/Pt

MFS/Sodiam Peroxide Fusion/Ny

MFVG/Cisito w HNOB/HCl/

MFVG Cesium Catbonate Fusion

MFVG/Sodium Peroxide Fusion/N

0.32354
0.124582
795.6106
239.91
23
DF
5
17
22
$\quad$ Num

$\begin{array}{rr}\text { Sum of Squares } & \text { Mean Squa } \\ 5146795 & 1029359 \\ 10760935 & 632996 \\ 15907730 & \\ & \\ \text { Mean } & \text { Std Error } \\ 73.97 & 397.81 \\ 67.47 & 397.81 \\ 1460.00 & 459.35 \\ 60.80 & 355.81 \\ 1.93 & 459.35 \\ 65.59 & 397.81 \\ & \\ & \\ \text { Mean } & \text { Std Dev } \\ 73.97 & 84.68 \\ 67.47 & 75.67 \\ 1460.00 & 2312.40 \\ 60.80 & 53.91 \\ 1.93 & 0.005774 \\ 65.59 & 73.51\end{array}$

$\begin{array}{rr}\text { Mean Square } & \text { F Ratio } \\ 1029359 & 1.6262 \\ 632996 & \\ & \\ & \\ \text { d Error } & \text { Lower } 95 \% \\ 397.81 & -765.3 \\ 397.81 & -771.8 \\ 459.35 & 490.9 \\ 355.81 & -689.9 \\ 459.35 & -967.2 \\ 397.81 & -773.7 \\ & \\ \text { Std Dev } & \text { Std Err Mean } \\ 84.68 & 42.3 \\ 75.67 & 37.8 \\ 2312.40 & 1335.1 \\ 53.91 & 24.1 \\ 0.005774 & 0.00333 \\ 73.51 & 36.8\end{array}$

Prob $>$ F

0.2066

Upper 95\%

913.3

906.8

2429.1
811.5
971.1

971.1

$\begin{array}{rr}\text { Number } & \text { Mean } \\ 4 & 73.97 \\ 4 & 67.47 \\ 3 & 1460.00 \\ 5 & 60.80 \\ 3 & 1.93 \\ 4 & 65.59\end{array}$

Lower $95 \%$
-61
-53
-4284
-6.1321
1.91232
-51

Upper 95\%

208.7

187.9

7204.3
127.7

1.9
182.6 
WSRC-TR-2005-00166

SRNL-RPP-2005-00029

Revision 0

Exhibit A2. Statistical Comparisons of Preparation/Crucible Measurements by Element by Type of Sample

Oneway Analysis of Zn By Simulant/Preparation/Crucible

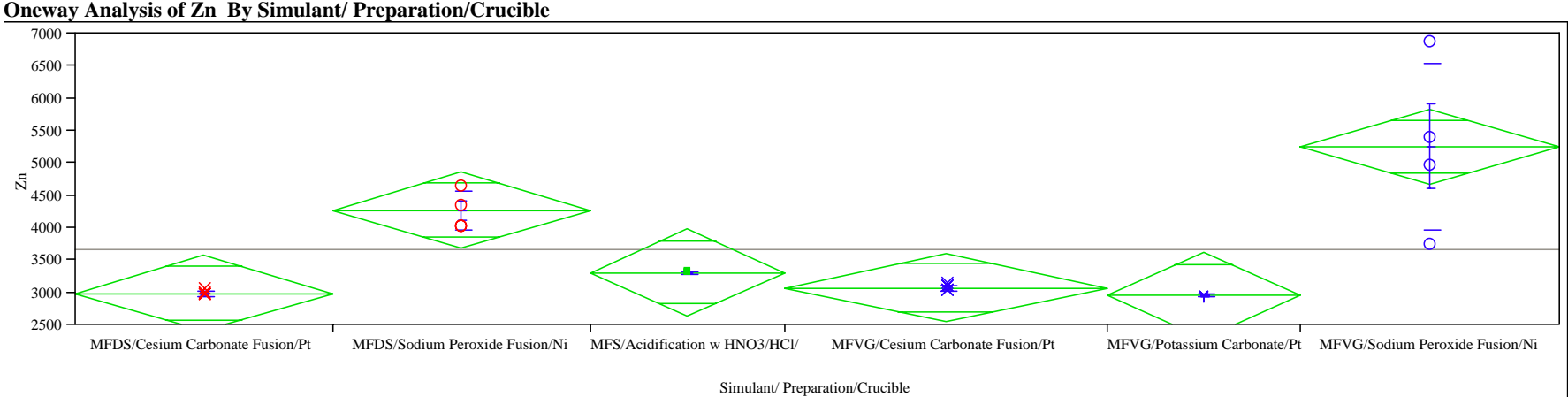

Oneway Anova

Summary of Fit

Rsquare

Adj Rsquare

Root Mean Square Erro

Mean of Response

Observations (or Sum Wgts)

Analysis of Variance

Simulant/ Preparation/Crucible

Error

Means for Oneway Anova

Means

MFDS/Cesium Carbonate Fusion/Pt

MFS/Sodium Peroxide Fusion/Ny

MFVG/Cesiaton w HNO3/HCl/

MFVG/Cesium Carbonate Fusion

MFVG/Sodium Peroxide Fusion

Ste

Ster error variance

Preparation/Crucible

Level

MFDS/Cesium Carbonate Fusion/Pt

MFDS/Sodium Peroxide Fusion/

MFS/Acidification w HNO3/HCl/

MFVG/Cesium Carbonate Fusion/

MFVG/Sodium Peroide Fusion

\begin{tabular}{|c|c|c|c|c|c|}
\hline $\begin{array}{r}0.76307 \\
0.693385 \\
557.5521 \\
3661 \\
23\end{array}$ & & & & & \\
\hline DF & Sum of Squares & Mean Square & F Ratio & Prob $>F$ & \\
\hline 5 & 17020208 & 3404042 & 10.9502 & $<.0001$ & \\
\hline 17 & 5284694 & 310864 & & & \\
\hline 22 & 22304902 & & & & \\
\hline Number & Mean & Std Error & Lower 95\% & Upper 95\% & \\
\hline 4 & 2988.25 & 278.78 & 2400.1 & 3576.4 & \\
\hline 4 & 4270.00 & 278.78 & 3681.8 & 4858.2 & \\
\hline 3 & 3310.00 & 321.90 & 2630.8 & 3989.2 & \\
\hline 5 & 3074.00 & 249.34 & 2547.9 & 3600.1 & \\
\hline 3 & 2953.33 & 321.90 & 2274.2 & 3632.5 & \\
\hline 4 & 5252.50 & 278.78 & 4664.3 & 5840.7 & \\
\hline Number & Mean & Std Dev & Std Err Mean & Lower 95\% & Upper 95\% \\
\hline 4 & 2988.25 & 38.44 & 19.22 & 2927.1 & 3049.4 \\
\hline 4 & 4270.00 & 299.44 & 149.72 & 3793.5 & 4746.5 \\
\hline 3 & 3310.00 & 17.32 & 10.00 & 3267.0 & 3353.0 \\
\hline 5 & 3074.00 & 43.36 & 19.39 & 3020.2 & 3127.8 \\
\hline 3 & 2953.33 & 20.82 & 12.02 & 2901.6 & 3005.0 \\
\hline 4 & 5252.50 & 1291.29 & 645.64 & 3197.8 & 7307.2 \\
\hline
\end{tabular}


WSRC-TR-2005-00166

SRNL-RPP-2005-00029

Revision 0

Exhibit A2. Statistical Comparisons of Preparation/Crucible Measurements by Element by Type of Sample

Oneway Analysis of Zr By Simulant/ Preparation/Crucible

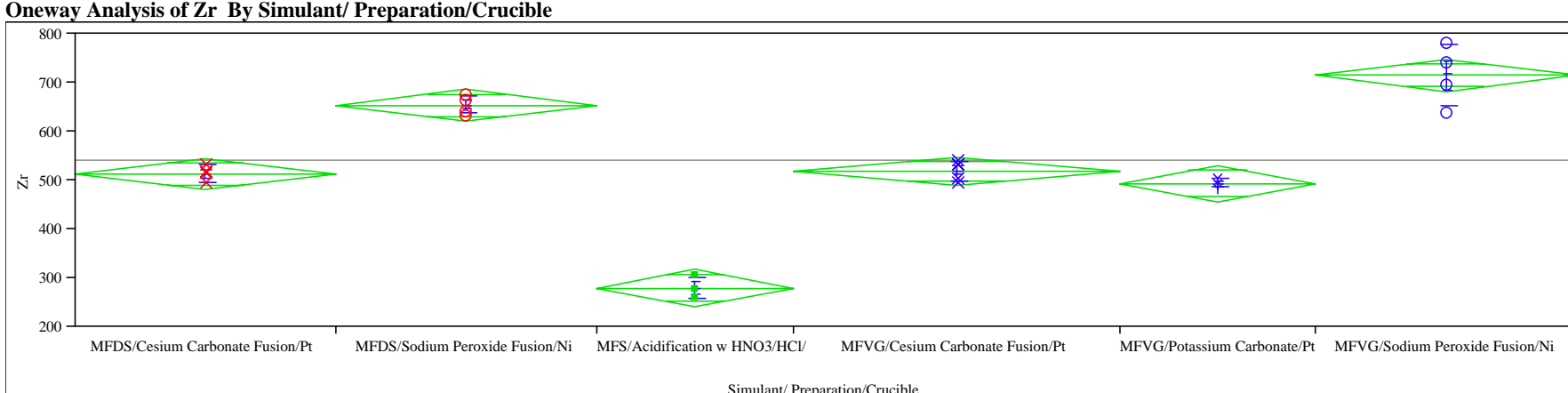

Simulant/ Preparation/Crucible

Oneway Anova

Summary of Fit

Rsquare

Adj Rsquare
Root Mean Square Error

Root Mean Square

Mean of Response
Observations (or Sum Wgts)

Analysis of Variance

Source

Simulant/ Preparation/Crucible

Error

Means for Oneway Anova

Means

MFDS/Cesium Carbonate Fusion/Pt

MFS/Sodin Peroxide Fusion/N/

MFVG/C ication w HNO3/HCl/

MFVG/Cesium Carbonate Fusion/Pt

MFVG/Sodium Perobonate Fusio

MFision/Ni



Means and Std Deviation

Level

MFDS/Cesium Carbonate Fusion/Pt

MFS/Aodian Peroxide Fusion/N/

MFVG/Cesical w

MFV/Posiun Carbonate Fusion/Pt

MFVG/Sodium Peroide Fusion

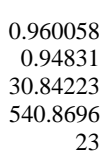

0.960058

0.94831

30.84223

23

\begin{tabular}{|c|c|c|}
\hline DF & Sum of Squares & Mean Squa \\
\hline 5 & 388691.48 & 77738 \\
\hline 17 & 16171.13 & 951 \\
\hline 22 & 404862.61 & \\
\hline Number & Mean & Std Error \\
\hline 4 & 513.000 & 15.421 \\
\hline 4 & 654.000 & 15.421 \\
\hline 3 & 279.667 & 17.807 \\
\hline 5 & 518.800 & 13.793 \\
\hline 3 & 493.667 & 17.807 \\
\hline 4 & 714.500 & 15.421 \\
\hline Number & Mean & Std Dev \\
\hline 4 & 513.000 & 16.0208 \\
\hline 4 & 654.000 & 19.3907 \\
\hline 3 & 279.667 & 23.1157 \\
\hline 5 & 518.800 & 19.9424 \\
\hline 3 & 493.667 & 10.5040 \\
\hline 4 & 714.500 & 61.6252 \\
\hline
\end{tabular}

Prob $>$ F

$\begin{array}{rrr} & \text { F Ratio } & \text { Prob }>\text { F } \\ & 81.7228 & <.0001\end{array}$

Lower 95\% Upper 95\%

$480.46 \quad 545.54$

621.46
24.10

242.10
489.70

489.70
456.10

$\begin{array}{ll}456.10 & 531.24 \\ 681.96 & 747.04\end{array}$

Std Err Mean

8.010

9.695

13.346
8.919

6.064

Lower $95 \%$
487.51
623.15
222.24
494.04
467.57
616.44

Upper $95 \%$

538.49

684.85

337.09

543.56
519.76

519.76
812.56 
WSRC-TR-2005-00166

SRNL-RPP-2005-00029

Revision 0

\section{Exhibit A3. All-Pairs Comparisons Among the Preparation Methods for Samples of the Dried Solids Form of the Melter Feed Slurry Simulant}

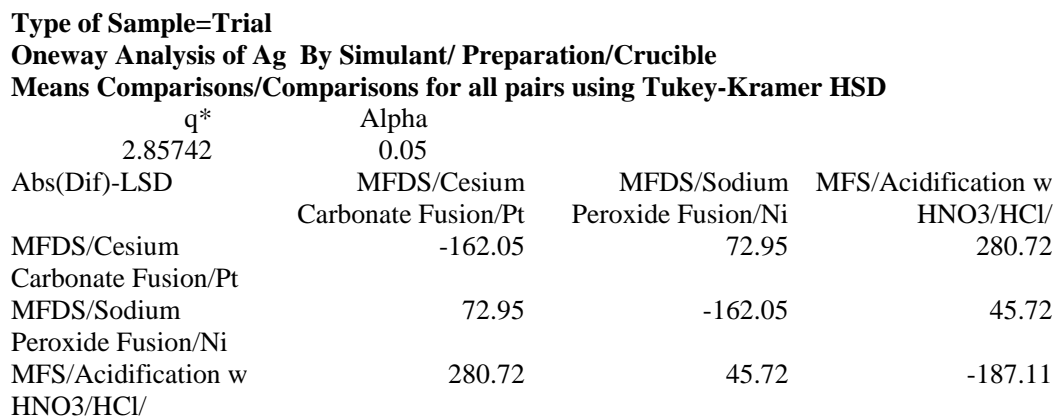

Positive values show pairs of means that are significantly different.

Oneway Analysis of Al By Simulant/ Preparation/Crucible

Means Comparisons/Comparisons for all pairs using Tukey-Kramer HSD

$\begin{array}{rr}\mathrm{q}^{*} & \text { Alpha } \\ 2.85742 & 0.05\end{array}$

Abs(Dif)-LSD

MFDS/Sodium

Peroxide Fusion/Ni

MFDS/Cesium

Carbonate Fusion/Pt

MFS/Acidification w

$\mathrm{HNO} / \mathrm{HCl} /$
MFDS/Sodium

Peroxide Fusion/Ni

$-514.0$

$-489.0$

2514.8

MFDS/Cesium
Carbonate Fusion/Pt
-489.0

MFS/Acidification w

2514.8

2489.8

$-593.5$

Positive values show pairs of means that are significantly different.

Oneway Analysis of B By Simulant/ Preparation/Crucible

Means Comparisons/Comparisons for all pairs using Tukey-Kramer HSD

\begin{tabular}{|c|c|c|c|}
\hline $\begin{array}{r}\mathrm{q}^{*} \\
2.85742\end{array}$ & $\begin{array}{r}\text { Alpha } \\
0.05\end{array}$ & & \\
\hline Abs(Dif)-LSD & $\begin{array}{r}\text { MFS/Acidification w } \\
\text { HNO3/HCl/ }\end{array}$ & $\begin{array}{r}\text { MFDS/Cesium } \\
\text { Carbonate Fusion/Pt }\end{array}$ & $\begin{array}{r}\text { MFDS/Sodium } \\
\text { Peroxide Fusion/Ni }\end{array}$ \\
\hline $\begin{array}{l}\text { MFS/Acidification w } \\
\mathrm{HNO3/HCl} /\end{array}$ & -247.5 & 618.5 & 1268.5 \\
\hline $\begin{array}{l}\text { MFDS/Cesium } \\
\text { Carbonate Fusion/Pt }\end{array}$ & 618.5 & -214.3 & 435.7 \\
\hline MFDS/Sodiu & 1268.5 & 435.7 & -214.3 \\
\hline
\end{tabular}

Peroxide Fusion/Ni

Positive values show pairs of means that are significantly different.

Oneway Analysis of Ba By Simulant/ Preparation/Crucible

Means Comparisons/Comparisons for all pairs using Tukey-Kramer HSD

\begin{tabular}{|c|c|c|c|}
\hline $\mathrm{q}^{*}$ & Alpha & & \\
\hline 2.85742 & 0.05 & & \\
\hline Abs(Dif)-LSD & $\begin{array}{r}\text { MFDS/Sodium } \\
\text { Peroxide Fusion/Ni }\end{array}$ & $\begin{array}{r}\text { MFDS/Cesium } \\
\text { Carbonate Fusion/Pt }\end{array}$ & $\begin{array}{r}\text { MFS/Acidification w } \\
\mathrm{HNO} / \mathrm{HCl} /\end{array}$ \\
\hline $\begin{array}{l}\text { MFDS/Sodium } \\
\text { Peroxide Fusion/Ni }\end{array}$ & -26.351 & 63.649 & 90.205 \\
\hline $\begin{array}{l}\text { MFDS/Cesium } \\
\text { Carbonate Fusion/Pt }\end{array}$ & 63.649 & -26.351 & 0.205 \\
\hline MFS/Acidification w & 90.205 & 0.205 & -30.427 \\
\hline
\end{tabular}

$\mathrm{HNO} / \mathrm{HCl} /$

Positive values show pairs of means that are significantly different. 
WSRC-TR-2005-00166

SRNL-RPP-2005-00029

Revision 0

\section{Exhibit A3. All-Pairs Comparisons Among the Preparation Methods for Samples of the Dried Solids Form of the Melter Feed Slurry Simulant}

\begin{tabular}{|c|c|c|c|}
\hline \multicolumn{4}{|c|}{$\begin{array}{l}\text { Oneway Analysis of Ca By Simulant/ Preparation/Crucible } \\
\text { Means Comparisons/Comparisons for all pairs using Tukey-Kramer HSD }\end{array}$} \\
\hline $\mathrm{q}^{*}$ & Alpha & & \\
\hline 2.85742 & 0.05 & & \\
\hline Abs(Dif)-LSD & $\begin{array}{r}\text { MFDS/Sodium } \\
\text { Peroxide Fusion/Ni }\end{array}$ & $\begin{array}{r}\text { MFS/Acidification w } \\
\text { HNO3/HCl/ }\end{array}$ & $\begin{array}{r}\text { MFDS/Cesium } \\
\text { Carbonate Fusion/Pt }\end{array}$ \\
\hline $\begin{array}{l}\text { MFDS/Sodium } \\
\text { Peroxide Fusion/Ni }\end{array}$ & -191.17 & 234.35 & 288.83 \\
\hline $\begin{array}{l}\text { MFS/Acidification w } \\
\text { HNO3/HCl/ }\end{array}$ & 234.35 & -220.75 & -167.32 \\
\hline $\begin{array}{l}\text { MFDS/Cesium } \\
\text { Carbonate Fusion/Pt }\end{array}$ & 288.83 & -167.32 & -191.17 \\
\hline
\end{tabular}

Positive values show pairs of means that are significantly different.

Oneway Analysis of Cd By Simulant/ Preparation/Crucible

Means Comparisons/Comparisons for all pairs using Tukey-Kramer HSD

Abs(Dif)-LSD

MFDS/Cesium MFDS/Sodium MFS/Acidification w

Carbonate Fusion/Pt Peroxide Fusion/Ni

$\begin{array}{lll}-7.6252 & -3.0002 & -2.4736\end{array}$

MFDS/Cesium

$\begin{array}{lll}-3.0002 & -7.6252 & -7.0986\end{array}$

MFDS/Sodium

Peroxide Fusion/Ni

MFS/Acidification w

$-2.4736$

$-7.0986$

$-8.8048$

$\mathrm{HNO} / \mathrm{HCl} /$

Positive values show pairs of means that are significantly different.

Oneway Analysis of Ce By Simulant/ Preparation/Crucible

Means Comparisons/Comparisons for all pairs using Tukey-Kramer HSD

\begin{tabular}{|c|c|c|c|}
\hline $\begin{array}{r}\mathrm{q}^{*} \\
2.85742\end{array}$ & $\begin{array}{r}\text { Alpha } \\
0.05\end{array}$ & & \\
\hline Abs(Dif)-LSD & $\begin{array}{r}\text { MFDS/Sodium } \\
\text { Peroxide Fusion/Ni }\end{array}$ & $\begin{array}{r}\text { MFS/Acidification w } \\
\mathrm{HNO} / \mathrm{HCl} /\end{array}$ & $\begin{array}{r}\text { MFDS/Cesium } \\
\text { Carbonate Fusion/Pt }\end{array}$ \\
\hline $\begin{array}{l}\text { MFDS/Sodium } \\
\text { Peroxide Fusion/Ni }\end{array}$ & -138.95 & -119.33 & -8.20 \\
\hline $\begin{array}{l}\text { MFS/Acidification w } \\
\mathrm{HNO} / \mathrm{HCl} /\end{array}$ & -119.33 & -160.45 & -50.08 \\
\hline MFDS/Cesium & -8.20 & -50.08 & -138.95 \\
\hline
\end{tabular}

Carbonate Fusion/Pt

Positive values show pairs of means that are significantly different.

Oneway Analysis of Cr By Simulant/ Preparation/Crucible

Means Comparisons/Comparisons for all pairs using Tukey-Kramer HSD

\begin{tabular}{lrrr}
\multicolumn{1}{c}{$\mathrm{q}^{*}$} & \multicolumn{1}{c}{ Alpha } & & \\
2.85742 & 0.05 & & \\
Abs(Dif)-LSD & MFDS/Sodium & MFDS/Cesium & MFS/Acidification w \\
& Peroxide Fusion/Ni & Carbonate Fusion/Pt & HNO3/HCl/ \\
MFDS/Sodium & -164.51 & 346.24 & 368.81 \\
$\begin{array}{l}\text { Peroxide Fusion/Ni } \\
\text { MFDS/Cesium }\end{array}$ & 346.24 & -164.51 & -141.94 \\
Carbonate Fusion/Pt & & & -189.96 \\
MFS/Acidification w & 368.81 & -141.94 &
\end{tabular}

$\mathrm{HNO} 3 / \mathrm{HCl} /$

Positive values show pairs of means that are significantly different. 
WSRC-TR-2005-00166

SRNL-RPP-2005-00029

Revision 0

\section{Exhibit A3. All-Pairs Comparisons Among the Preparation Methods for Samples of the Dried Solids Form of the Melter Feed Slurry Simulant}

\begin{tabular}{|c|c|c|c|}
\hline \multicolumn{4}{|c|}{$\begin{array}{l}\text { Oneway Analysis of Cu By Simulant/ Preparation/Crucible } \\
\text { Means Comparisons/Comparisons for all pairs using Tukey-Kramer HSD }\end{array}$} \\
\hline $\mathrm{q}^{*}$ & Alpha & & \\
\hline 2.85742 & 0.05 & & \\
\hline Abs(Dif)-LSD & $\begin{array}{r}\text { MFDS/Cesium } \\
\text { Carbonate Fusion/Pt }\end{array}$ & $\begin{array}{r}\text { MFS/Acidification w } \\
\text { HNO3/HCl/ }\end{array}$ & $\begin{array}{r}\text { MFDS/Sodium } \\
\text { Peroxide Fusion/Ni }\end{array}$ \\
\hline $\begin{array}{l}\text { MFDS/Cesium } \\
\text { Carbonate Fusion/Pt }\end{array}$ & -9.371 & 18.278 & 37.929 \\
\hline $\begin{array}{l}\text { MFS/Acidification w } \\
\text { HNO3/HCl/ }\end{array}$ & 18.278 & -10.821 & 8.778 \\
\hline MFDS/Sodium & 37.929 & 8.778 & -9.371 \\
\hline
\end{tabular}

Peroxide Fusion/Ni

Positive values show pairs of means that are significantly different.

Oneway Analysis of Fe By Simulant/ Preparation/Crucible

Means Comparisons/Comparisons for all pairs using Tukey-Kramer HSD

$\begin{array}{rr}\mathrm{q}^{*} & \text { Alpha } \\ 2.85742 & 0.05\end{array}$

Abs(Dif)-LSD

MFS/Acidification w

MFDS/Sodium

MFDS/Cesium $\mathrm{HNO} / \mathrm{HCl} /$

Peroxide Fusion/Ni

Carbonate Fusion/Pt

$\begin{array}{lrr}-657.31 & -89.86 & 760.14\end{array}$

$\mathrm{HNO} / \mathrm{HCl}$

MFDS/Sodium

$-89.86$

$-569.25$

280.75

Peroxide Fusion/N

MFDS/Cesium

760.14

280.75

$-569.25$

Carbonate Fusion/Pt

Positive values show pairs of means that are significantly different.

Oneway Analysis of K By Simulant/ Preparation/Crucible

Means Comparisons/Comparisons for all pairs using Tukey-Kramer HSD

2.85742

Abs(Dif)-LSD

MFDS/Sodium

Peroxide Fusion/Ni

MFDS/Cesium

Carbonate Fusion/Pt

MFS/Acidification w

$\mathrm{HNO} / \mathrm{HCl} /$

\begin{tabular}{rrr}
\multicolumn{1}{c}{ Alpha } & & \\
0.05 & & \\
MFDS/Sodium & MFDS/Cesium & MFS/Acidification w \\
Peroxide Fusion/Ni & Carbonate Fusion/Pt & HNO3/HCl/ \\
-266.54 & 413.46 & 782.94 \\
413.46 & -266.54 & 102.94 \\
782.94 & 102.94 & -307.77
\end{tabular}

Positive values show pairs of means that are significantly different.

Oneway Analysis of La By Simulant/ Preparation/Crucible

Means Comparisons/Comparisons for all pairs using Tukey-Kramer HSD

\begin{tabular}{|c|c|c|c|}
\hline $\mathrm{q}^{*}$ & Alpha & & \\
\hline 2.85742 & 0.05 & & \\
\hline Abs(Dif)-LSD & $\begin{array}{r}\text { MFS/Acidification w } \\
\mathrm{HNO} / \mathrm{HCl} /\end{array}$ & $\begin{array}{r}\text { MFDS/Sodium } \\
\text { Peroxide Fusion/Ni }\end{array}$ & $\begin{array}{r}\text { MFDS/Cesium } \\
\text { Carbonate Fusion/Pt }\end{array}$ \\
\hline $\begin{array}{l}\text { MFS/Acidification w } \\
\mathrm{HNO} / \mathrm{HCl} /\end{array}$ & -38.426 & -17.028 & 7.472 \\
\hline $\begin{array}{l}\text { MFDS/Sodium } \\
\text { Peroxide Fusion/Ni }\end{array}$ & -17.028 & -33.278 & -8.778 \\
\hline MFDS/Cesium & 7.472 & -8.778 & -33.278 \\
\hline
\end{tabular}

Carbonate Fusion/Pt

Positive values show pairs of means that are significantly different. 
WSRC-TR-2005-00166

SRNL-RPP-2005-00029

Revision 0

\section{Exhibit A3. All-Pairs Comparisons Among the Preparation Methods for Samples of the Dried Solids Form of the Melter Feed Slurry Simulant}

\begin{tabular}{|c|c|c|c|}
\hline \multicolumn{4}{|c|}{$\begin{array}{l}\text { Oneway Analysis of } \mathrm{Li} \text { By Simulant/ Preparation/Crucible } \\
\text { Means Comparisons/Comparisons for all pairs using Tukey-Kramer HSD }\end{array}$} \\
\hline$q^{*}$ & Alpha & & \\
\hline 2.85742 & 0.05 & & \\
\hline Abs(Dif)-LSD & $\begin{array}{r}\text { MFS/Acidification w } \\
\text { HNO3/HCl/ }\end{array}$ & $\begin{array}{r}\text { MFDS/Sodium } \\
\text { Peroxide Fusion/Ni }\end{array}$ & $\begin{array}{r}\text { MFDS/Cesium } \\
\text { Carbonate Fusion/Pt }\end{array}$ \\
\hline $\begin{array}{l}\text { MFS/Acidification w } \\
\text { HNO3/HCl/ }\end{array}$ & -133.18 & -81.24 & 298.76 \\
\hline $\begin{array}{l}\text { MFDS/Sodium } \\
\text { Peroxide Fusion/Ni }\end{array}$ & -81.24 & -115.33 & 264.67 \\
\hline $\begin{array}{l}\text { MFDS/Cesium } \\
\text { Carbonate Fusion/Pt }\end{array}$ & 298.76 & 264.67 & -115.33 \\
\hline
\end{tabular}

Positive values show pairs of means that are significantly different.

Oneway Analysis of Mg By Simulant/Preparation/Crucible

Means Comparisons/Comparisons for all pairs using Tukey-Kramer HSD

2.85742

Abs(Dif)-LSD

MFDS/Sodium

Peroxide Fusion/Ni

MFDS/Cesium

Carbonate Fusion/Pt

MFS/Acidification w

$\mathrm{HNO} / \mathrm{HCl} /$

Positive values show pairs of means that are significantly different.
MFDS/Cesium MFS/Acidification w

Carbonate Fusion/Pt $\quad \mathrm{HNO} / \mathrm{HCl} /$

$\begin{array}{ll}-12.159 & 3.303\end{array}$

$\begin{array}{rrr}-18.159 & -12.159 & 3.303 \\ -12.159 & -18.159 & -2.697\end{array}$

$\begin{array}{lll}3.303 & -2.697 & -20.968\end{array}$

Oneway Analysis of Mn By Simulant/Preparation/Crucible

Means Comparisons/Comparisons for all pairs using Tukey-Kramer HSD

q* Alpha

Abs(Dif)-LSD

MFS/Acidification w $\mathrm{HNO} / \mathrm{HCl} /$

$-147.19$

MFDS/Sodium

Peroxide Fusion/Ni

133.98

MFDS/Cesium

MFS/Acidification w

133.98

$-127.47$

Carbonate Fusion/Pt

MFDS/Sodium

366.48

105.03

MFDS/Cesium

366.48

$-127.47$

Carbonate Fusion/Pt

Positive values show pairs of means that are significantly different.

Oneway Analysis of Mo By Simulant/ Preparation/Crucible

Means Comparisons/Comparisons for all pairs using Tukey-Kramer HSD

\begin{tabular}{|c|c|c|c|}
\hline $\begin{array}{r}\mathrm{q}^{*} \\
285742\end{array}$ & Alpha & & \\
\hline Abs(Dif)-LSD & $\begin{array}{r}\text { MFDS/Sodium } \\
\text { Peroxide Fusion/Ni }\end{array}$ & $\begin{array}{r}\text { MFS/Acidification w } \\
\mathrm{HNO} / \mathrm{HCl} /\end{array}$ & $\begin{array}{r}\text { MFDS/Cesium } \\
\text { Carbonate Fusion/Pt }\end{array}$ \\
\hline $\begin{array}{l}\text { MFDS/Sodium } \\
\text { Peroxide Fusion/Ni }\end{array}$ & -193.89 & 551.33 & 586.49 \\
\hline $\begin{array}{l}\text { MFS/Acidification w } \\
\mathrm{HNO} / \mathrm{HCl} /\end{array}$ & 551.33 & -223.88 & -189.80 \\
\hline MFDS/Cesium & 586.49 & -189.80 & -193.89 \\
\hline
\end{tabular}

Carbonate Fusion/Pt

Positive values show pairs of means that are significantly different. 
WSRC-TR-2005-00166

SRNL-RPP-2005-00029

Revision 0

\section{Exhibit A3. All-Pairs Comparisons Among the Preparation Methods for Samples of the Dried Solids Form of the Melter Feed Slurry Simulant}

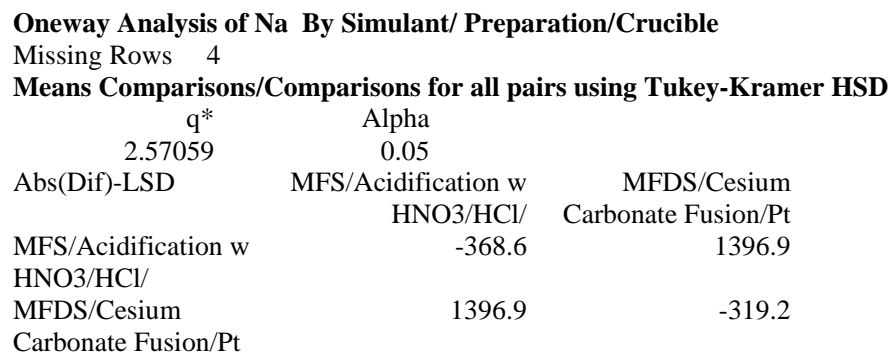

Positive values show pairs of means that are significantly different.

Oneway Analysis of Ni By Simulant/Preparation/Crucible

Missing Rows 4

Means Comparisons/Comparisons for all pairs using Tukey-Kramer HSD

q* Alpha

$\begin{array}{lrr}\text { Abs(Dif)-LSD } & \text { MFS/Acidification w } & \begin{array}{r}\text { MFDS/Cesium } \\ \text { HNO3/HCl/ }\end{array} \\ \text { Carbonate Fusion/Pt } \\ \text { MFS/Acidification w } & -60.502 & -21.928 \\ \text { HNO3/HCl/ } & & \\ \text { MFDS/Cesium } & -21.928 & -52.396\end{array}$

Carbonate Fusion/Pt

Positive values show pairs of means that are significantly different.

Oneway Analysis of $\mathbf{P}$ By Simulant/ Preparation/Crucible

Means Comparisons/Comparisons for all pairs using Tukey-Kramer HSD

\begin{tabular}{|c|c|c|c|}
\hline $\begin{array}{r}q^{*} \\
2.85742\end{array}$ & $\begin{array}{r}\text { Alpha } \\
0.05\end{array}$ & & \\
\hline Abs(Dif)-LSD & MFDS/Cesium & MFDS/Sodium & MFS/Acidification w \\
\hline & Carbonate Fusion/Pt & Peroxide Fusion/Ni & $\mathrm{HNO} / \mathrm{HCl} /$ \\
\hline $\begin{array}{l}\text { MFDS/Cesium } \\
\text { Carbonate Fusion/Pt }\end{array}$ & -267.11 & -100.86 & -81.42 \\
\hline $\begin{array}{l}\text { MFDS/Sodium } \\
\text { Peroxide Fusion/Ni }\end{array}$ & -100.86 & -267.11 & -247.67 \\
\hline MFS/Acidification w & -81.42 & -247.67 & -308.43 \\
\hline
\end{tabular}

$\mathrm{HNO} / \mathrm{HCl}$

Positive values show pairs of means that are significantly different.

Oneway Analysis of $\mathbf{P b}$ By Simulant/ Preparation/Crucible

Means Comparisons/Comparisons for all pairs using Tukey-Kramer HSD

\begin{tabular}{|c|c|c|c|}
\hline $\begin{array}{r}\mathrm{q}^{*} \\
2.85742\end{array}$ & $\begin{array}{r}\text { Alpha } \\
0.05\end{array}$ & & \\
\hline Abs(Dif)-LSD & $\begin{array}{r}\text { MFDS/Sodium } \\
\text { Peroxide Fusion/Ni }\end{array}$ & $\begin{array}{r}\text { MFS/Acidification w } \\
\text { HNO3/HCl/ }\end{array}$ & $\begin{array}{r}\text { MFDS/Cesium } \\
\text { Carbonate Fusion/Pt }\end{array}$ \\
\hline $\begin{array}{l}\text { MFDS/Sodium } \\
\text { Peroxide Fusion/Ni }\end{array}$ & -626.49 & -218.36 & 612.26 \\
\hline $\begin{array}{l}\text { MFS/Acidification w } \\
\mathrm{HNO} / \mathrm{HCl} /\end{array}$ & -218.36 & -723.41 & 103.73 \\
\hline MFDS/Cesium & 612.26 & 103.73 & -626.49 \\
\hline
\end{tabular}

Carbonate Fusion/Pt

Positive values show pairs of means that are significantly different. 
WSRC-TR-2005-00166

SRNL-RPP-2005-00029

Revision 0

\section{Exhibit A3. All-Pairs Comparisons Among the Preparation Methods for Samples of the Dried Solids Form of the Melter Feed Slurry Simulant}

\begin{tabular}{|c|c|c|c|}
\hline \multicolumn{4}{|c|}{ Oneway Analysis of S By Simulant/ Preparation/Crucible } \\
\hline Missing Rows 7 & & & \\
\hline \multicolumn{4}{|c|}{$\begin{array}{l}\text { Oneway Analysis of Sb By Simulant/ Preparation/Crucible } \\
\text { Means Comparisons/Comparisons for all pairs using Tukey-Kramer HSD }\end{array}$} \\
\hline $\mathrm{q}^{*}$ & Alpha & & \\
\hline 2.85742 & 0.05 & & \\
\hline Abs(Dif)-LSD & MFDS/Sodium & MFDS/Cesium & MFS/Acidification w \\
\hline & Peroxide Fusion/Ni & Carbonate Fusion/Pt & $\mathrm{HNO} / \mathrm{HCl} /$ \\
\hline MFDS/Sodium & -514.4 & 1161.1 & 1137.1 \\
\hline Peroxide Fusion/Ni & & & \\
\hline MFDS/Cesium & 1161.1 & -514.4 & -538.4 \\
\hline Carbonate Fusion/Pt & & & \\
\hline MFS/Acidification w & 1137.1 & -538.4 & -593.9 \\
\hline
\end{tabular}

$\mathrm{HNO} / \mathrm{HCl} /$

Positive values show pairs of means that are significantly different.

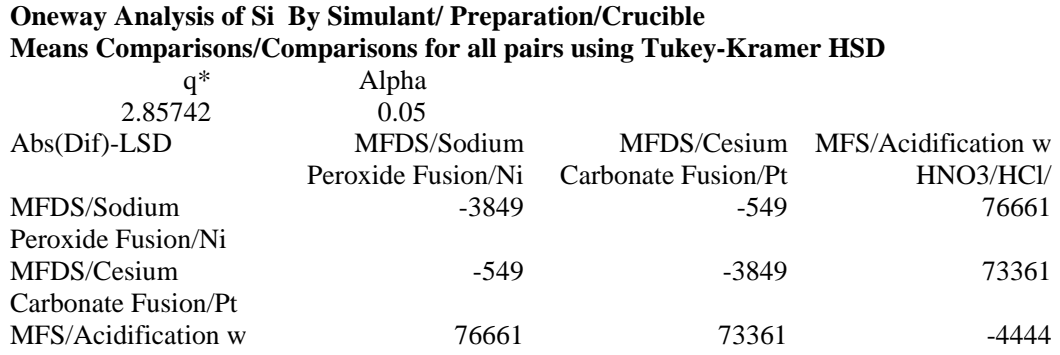

$\mathrm{HNO} 3 / \mathrm{HCl} /$

Positive values show pairs of means that are significantly different.

Oneway Analysis of Sn By Simulant/ Preparation/Crucible

Means Comparisons/Comparisons for all pairs using Tukey-Kramer HSD

\begin{tabular}{|c|c|c|c|}
\hline $\mathrm{q}^{*}$ & Alpha & & \\
\hline 2.85742 & 0.05 & & \\
\hline \multirow[t]{2}{*}{ Abs(Dif)-LSD } & MFDS/Cesium & MFDS/Sodium & MFS/Acidification w \\
\hline & Carbonate Fusion/Pt & Peroxide Fusion/Ni & $\mathrm{HNO} 3 / \mathrm{HCl} /$ \\
\hline MFDS/Cesium & -167.12 & -65.24 & -54.42 \\
\hline \multicolumn{4}{|l|}{ Carbonate Fusion/Pt } \\
\hline MFDS/Sodium & -65.24 & -167.12 & -156.30 \\
\hline \multicolumn{4}{|l|}{ Peroxide Fusion/Ni } \\
\hline MFS/Acidification w & -54.42 & -156.30 & -192.97 \\
\hline
\end{tabular}

$\mathrm{HNO} / \mathrm{HCl} /$

Positive values show pairs of means that are significantly different.

Oneway Analysis of Sr By Simulant/ Preparation/Crucible

Means Comparisons/Comparisons for all pairs using Tukey-Kramer HSD

\begin{tabular}{|c|c|c|c|}
\hline $\begin{array}{r}\mathrm{q}^{*} \\
2.85742\end{array}$ & $\begin{array}{r}\text { Alpha } \\
0.05\end{array}$ & & \\
\hline Abs(Dif)-LSD & $\begin{array}{r}\text { MFDS/Sodium } \\
\text { Peroxide Fusion/Ni }\end{array}$ & $\begin{array}{r}\text { MFS/Acidification w } \\
\mathrm{HNO} / \mathrm{HCl} /\end{array}$ & $\begin{array}{r}\text { MFDS/Cesium } \\
\text { Carbonate Fusion/Pt }\end{array}$ \\
\hline MFDS/Sodium & -48.341 & 72.452 & 88.909 \\
\hline Peroxide Fusion/Ni & & & \\
\hline $\begin{array}{l}\text { MFS/Acidification w } \\
\mathrm{HNO} / \mathrm{HCl} /\end{array}$ & 72.452 & -55.820 & -39.631 \\
\hline MFDS/Cesium & 88.909 & -39.631 & -48.341 \\
\hline
\end{tabular}

Carbonate Fusion/Pt

Positive values show pairs of means that are significantly different. 
WSRC-TR-2005-00166

SRNL-RPP-2005-00029

Revision 0

\section{Exhibit A3. All-Pairs Comparisons Among the Preparation Methods for Samples of the Dried Solids Form of the Melter Feed Slurry Simulant}

Oneway Analysis of Th By Simulant/ Preparation/Crucible

Missing Rows 7

\begin{tabular}{|c|c|c|c|}
\hline $\begin{array}{l}\text { Oneway Analysis of } \\
\text { Means Comparisons }\end{array}$ & $\begin{array}{l}\text { By Simulant/ Prepar } \\
\text { omparisons for all pa }\end{array}$ & $\begin{array}{l}\text { ion/Crucible } \\
\text { using Tukey-Kram }\end{array}$ & $r$ HSD \\
\hline$q^{*}$ & Alpha & & \\
\hline 2.85742 & 0.05 & & \\
\hline Abs(Dif)-LSD & MFDS/Cesium & MFDS/Sodium & MFS/Acidification w \\
\hline & Carbonate Fusion/Pt & Peroxide Fusion/Ni & $\mathrm{HNO} / \mathrm{HCl} /$ \\
\hline MFDS/Cesium & -2.049 & 6.764 & 13.105 \\
\hline Carbonate Fusion/Pt & & & \\
\hline MFDS/Sodium & 6.764 & -2.049 & 4.293 \\
\hline Peroxide Fusion/Ni & & & \\
\hline MFS/Acidification w & 13.105 & 4.293 & -2.366 \\
\hline
\end{tabular}

$\mathrm{HNO} / \mathrm{HCl} /$

Positive values show pairs of means that are significantly different.

Oneway Analysis of Tl By Simulant/ Preparation/Crucible

Missing Rows 7

Oneway Analysis of U By Simulant/Preparation/Crucible

Missing Rows 7

Oneway Analysis of Y By Simulant/ Preparation/Crucible

Means Comparisons/Comparisons for all pairs using Tukey-Kramer HSD

$$
\begin{array}{rr}
\mathrm{q}^{*} & \text { Alpha } \\
2.85742 & 0.05
\end{array}
$$

MFS/Acidification w MFDS/Cesium MFDS/Sodium $\mathrm{HNO} / \mathrm{HCl} / \quad$ Carbonate Fusion/Pt Peroxide Fusion/Ni

$\begin{array}{lccc}\text { MFS/Acidification w } & -2702.4 & -1141.8 & -1135.3 \\ \text { HNO3/HCl/ } & -1141.8 & -2340.3 & -2333.8 \\ \begin{array}{l}\text { MFDS/Cesium } \\ \text { Carbonate Fusion/Pt }\end{array} & -1135.3 & -2333.8 & -2340.3 \\ \text { MFDS/Sodium } & & & \end{array}$

Peroxide Fusion/Ni

Positive values show pairs of means that are significantly different.

Oneway Analysis of Zn By Simulant/ Preparation/Crucible

Means Comparisons/Comparisons for all pairs using Tukey-Kramer HSD

\begin{tabular}{|c|c|c|c|}
\hline $\begin{array}{r}\mathrm{q}^{*} \\
2.85742\end{array}$ & $\begin{array}{r}\text { Alpha } \\
0.05\end{array}$ & & \\
\hline Abs(Dif)-LSD & $\begin{array}{r}\text { MFDS/Sodium } \\
\text { Peroxide Fusion/Ni }\end{array}$ & $\begin{array}{r}\text { MFS/Acidification w } \\
\mathrm{HNO} / \mathrm{HCl} /\end{array}$ & $\begin{array}{r}\text { MFDS/Cesium } \\
\text { Carbonate Fusion/Pt }\end{array}$ \\
\hline $\begin{array}{l}\text { MFDS/Sodium } \\
\text { Peroxide Fusion/Ni }\end{array}$ & -373.95 & 556.09 & 907.80 \\
\hline $\begin{array}{l}\text { MFS/Acidification w } \\
\mathrm{HNO} / \mathrm{HCl} /\end{array}$ & 556.09 & -431.80 & -82.16 \\
\hline MFDS/Cesium & 907.80 & -82.16 & -373.95 \\
\hline
\end{tabular}

Carbonate Fusion/Pt

Positive values show pairs of means that are significantly different.

Oneway Analysis of Zr By Simulant/ Preparation/Crucible

Means Comparisons/Comparisons for all pairs using Tukey-Kramer HSD

\begin{tabular}{|c|c|c|c|}
\hline $\begin{array}{r}\mathrm{q}^{*} \\
2.85742\end{array}$ & $\begin{array}{r}\text { Alpha } \\
0.05\end{array}$ & & \\
\hline Abs(Dif)-LSD & $\begin{array}{r}\text { MFDS/Sodium } \\
\text { Peroxide Fusion/Ni }\end{array}$ & $\begin{array}{r}\text { MFDS/Cesium } \\
\text { Carbonate Fusion/Pt }\end{array}$ & $\begin{array}{r}\text { MFS/Acidification w } \\
\text { HNO3/HCl/ }\end{array}$ \\
\hline $\begin{array}{l}\text { MFDS/Sodium } \\
\text { Peroxide Fusion/Ni }\end{array}$ & -38.91 & 102.09 & 332.31 \\
\hline $\begin{array}{l}\text { MFDS/Cesium } \\
\text { Carbonate Fusion/Pt }\end{array}$ & 102.09 & -38.91 & 191.31 \\
\hline MFS/Acidification w & 332.31 & 191.31 & -44.93 \\
\hline
\end{tabular}

$\mathrm{HNO} 3 / \mathrm{HCl} /$

Positive values show pairs of means that are significantly different. 